Finite fault stochastic modeling of the 1999

\title{
Chi-Chi Taiwan Earthquake
}

by

Chengxiang Liu

\author{
A thesis submitted to \\ the Faculty of Graduate Studies and Research \\ in partial fulfillment of the requirements \\ for the degree of Master of Science
}

Department of Earth Sciences,

Carleton University

Ottawa, Ontario, Canada

May, 2006

(C) Carleton University

Chengxiang Liu 


$\begin{array}{ll}\begin{array}{l}\text { Library and } \\ \text { Archives Canada }\end{array} & \begin{array}{l}\text { Bibliothèque et } \\ \text { Archives Canada }\end{array} \\ \begin{array}{l}\text { Published Heritage } \\ \text { Branch }\end{array} & \begin{array}{l}\text { Direction du } \\ \text { Patrimoine de l'édition }\end{array} \\ \begin{array}{l}\text { 395 Wellington Street } \\ \text { Ottawa ON K1A 0N4 }\end{array} & \begin{array}{l}\text { 395, rue Wellington } \\ \text { Ottana ON K1A ON4 } \\ \text { Canada Oa }\end{array}\end{array}$

Your file Votre référence ISBN: 978-0-494-16499-0 Our file Notre référence ISBN: 978-0-494-16499-0

NOTICE:

The author has granted a nonexclusive license allowing Library and Archives Canada to reproduce, publish, archive, preserve, conserve, communicate to the public by telecommunication or on the Internet, loan, distribute and sell theses worldwide, for commercial or noncommercial purposes, in microform, paper, electronic and/or any other formats.

The author retains copyright ownership and moral rights in this thesis. Neither the thesis nor substantial extracts from it may be printed or otherwise reproduced without the author's permission.
AVIS:

L'auteur a accordé une licence non exclusive permettant à la Bibliothèque et Archives Canada de reproduire, publier, archiver, sauvegarder, conserver, transmettre au public par télécommunication ou par l'Internet, prêter, distribuer et vendre des thèses partout dans le monde, à des fins commerciales ou autres, sur support microforme, papier, électronique et/ou autres formats.

L'auteur conserve la propriété du droit d'auteur et des droits moraux qui protège cette thèse. $\mathrm{Ni}$ la thèse ni des extraits substantiels de celle-ci ne doivent être imprimés ou autrement reproduits sans son autorisation.
In compliance with the Canadian

Privacy Act some supporting forms may have been removed from this thesis.

While these forms may be included in the document page count, their removal does not represent any loss of content from the thesis.
Conformément à la loi canadienne sur la protection de la vie privée, quelques formulaires secondaires ont été enlevés de cette thèse.

Bien que ces formulaires aient inclus dans la pagination, il n'y aura aucun contenu manquant. 


\begin{abstract}
Ground motion prediction plays an important role in seismic hazard evaluation and mitigation. Among various techniques, the stochastic simulation method is a simple, powerful and widely used way to characterize the behavior of ground motions of a broad range of earthquake magnitudes and distances. By comparing the response spectra of observed and simulated ground motions, the source parameters of the earthquake, the propagation properties of the crust and the site amplification behavior may be inferred. This information allows us to better predict future ground motions and ultimately improve building codes.

In this study, the revised Finite Fault Stochastic Simulation technique proposed by Motazedian (2002) and Motazedian and Atkinson (2005) is used to model over 400 ground motion records from the $1999 M_{W}$ 7.6 Chi-Chi Taiwan earthquake, at nearfault to regional distances. The geometric and anelastic attenuation properties of the crust, site amplifications and duration are empirically calculated from the recorded seismic data.

With the specified path and site model for the Chi-Chi earthquake, stochastic simulation is conducted first on rock stations for model calibration, and then on soil stations using the calibrated model. Based on comparisons of simulations and data, I conclude that the stress drop of the Chi-Chi earthquake was 40 bars, which is relatively low compared with earthquakes of similar magnitude in other regions (eg. 50-70 bars in California). Regarding site effects, I conclude that the H/V (horizontal/vertical component) ratio works well as a site amplification term, and that obvious nonlinear soil response was observed on soil stations during the Chi-Chi earthquake.
\end{abstract}


Furthermore, there are marked basin effects in the Taipei region for ground motions of intermediate frequency (around $1 \mathrm{~Hz}$ ). This could be an important contributing reason for building damage in the Taipei area during the Chi-Chi earthquake. 


\section{ACKNOWLEDGMENTS}

In this section, I would like to give my hearty thanks to the following people. Without them, the completion of this thesis would be impossible.

I would like to extend my thanks to my supervisor Professor Gail Atkinson for her continuous guidance, encouragement and support. Her broad knowledge in earth sciences and seismology benefits me a lot during my study at Carleton University.

I also thank my co-supervisor Professor Dariush Motazedian who gave me much help in the process of this project. His advice made the thesis prominent. His contribution on the finite fault modeling made this project possible.

The fellow graduate students in the POLARIS Lab at Carleton University and the personnel in the department of Earth Sciences are owed my thanks as well. Discussions and cooperation with them made my research move forward.

Here, I thank the National Center for Research on Earthquake Engineering of Taiwan who provided me the shallow shear wave velocity information. I also thank Professor Wang, Wei-Hau and Mr. Chang, Shu-Hao of National Chung Cheng University for providing me the $2 \mathrm{D}$ fault slip distribution model.

Finally, I will give my best thanks to my dear wife Jing Wang for her continuous support in these two years. 


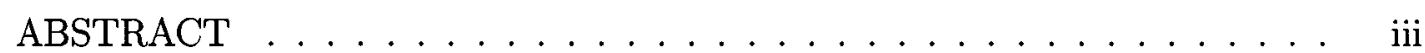

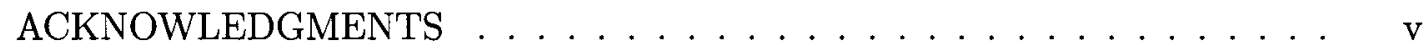

LIST OF TABLES . . . . . . . . . . . . . . . . . . . v viii

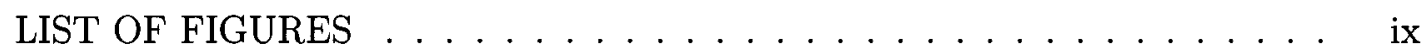

LIST OF ACRONYMS ................... xiv

Chapter 1 INTRODUCTION ......................... 1

1.1 Purpose of Study . . . . . . . . . . . . . . . . . . 1

1.2 Organization of Work . . . . . . . . . . . . . . 2

1.3 Contribution . . . . . . . . . . . . . . . . . 3

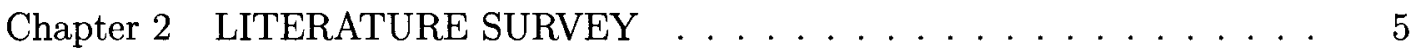

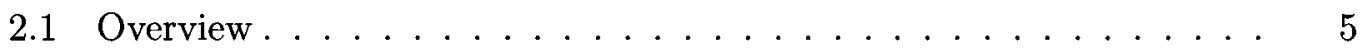

2.2 Stochastic Simulation . . . . . . . . . . . . . 6

2.2.1 Point Source Stochastic Simulation . . . . . . . . . . 6

2.2.2 Finite Fault Stochastic Simulation . . . . . . . . . . . . . 10

2.2.3 Refinements to the Finite Fault Stochastic Simulation . . . . . 11

2.3 Background of the Chi-Chi Earthquake . . . . . . . . . . . . 13

2.3.1 Tectonics and Seismicity of Taiwan . . . . . . . . . . . 13

2.3.2 Geological Setting. . . . . . . . . . . . . . . . . 14

Chapter 3 AVAILABLE DATA AND PROCESSING PROCEDURES . . . . 28

3.1 Introduction . . . . . . . . . . . . . . . . 28

3.2 Seismic Network and Data Set . . . . . . . . . . . . . . 28

3.3 Data Processing Procedures . . . . . . . . . . . . . . . . . . 29

Chapter 4 SEISMIC PARAMETERS OF THE CHI-CHI MAINSHOCK $\ldots 46$

4.1 Introduction . . . . . . . . . . . . . . . . . 46 
4.2 Geometric Parameters of the Main Shock . . . . . . . . . . . . . . 47

4.2 .1 Fault Plane . . . . . . . . . . . . . . . . . . 47

4.2 .2 Main Shock Location . . . . . . . . . . . . . . . . 47

4.3 Site Classification . . . . . . . . . . . . . . . . . . . . . 48

4.4 Kappa Factor . . . . . . . . . . . . . . . . . . . . . . . . 49

4.5 Wave Propagation Analysis . . . . . . . . . . . . . . 51

4.5 .1 Attenuation Regression . . . . . . . . . . . . . . 51

4.5 .2 Q model . . . . . . . . . . . . . . . . . 53

4.6 Site Amplification . . . . . . . . . . . . . . . . . . 53

$4.6 .1 \mathrm{H} / \mathrm{V}$ Ratio . . . . . . . . . . . . . . . . . 54

4.6.2 Quarter Wavelength Approximation Approach . . . . . . . . 54

4.7 Duration of Motions . . . . . . . . . . . . . 66

Chapter 5 SIMULATION OF THE CHI-CHI EARTHQUAKE GROUND MO-

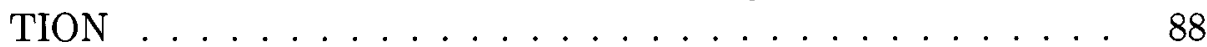

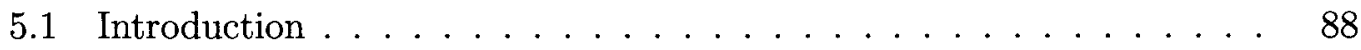

5.2 Input Model . . . . . . . . . . . . . . . . . . . . . 88

5.3 Results and Analysis . . . . . . . . . . . . . . . . . . 90

5.3.1 Simulation for Rock Stations . . . . . . . . . . . . . . 90

5.3.2 Simulation for linear behavior of soil stations . . . . . . . . . 94

5.3.3 Simulation for non-linear behavior of soil stations . . . . . . 95

Chapter 6 CONCLUSIONS AND FUTURE WORK $\ldots \ldots \ldots \ldots$

6.1 Conclusions . . . . . . . . . . . . . . . . . . . . 135

6.2 Future Work . . . . . . . . . . . . . . . . . . 136

REFERENCES . . . . . . . . . . . . . . . . . . . 138

APPENDIX A SAMPLE SIMULATED ACCELEROGRAMS . . . . . . . 143

vii 


\section{LIST OF TABLES}

3.1 Stations used in this study . . . . . . . . . . . . . . . . . . . 30

4.1 NEHRP site classification scheme . . . . . . . . . . . . . . . 49

4.2 Stations to calculate site amplification (NEHRP class B sites) . . . 55

4.3 Stations to calculate site amplification (NEHRP class C sites) . . . 56

4.4 Stations to calculate site amplification (NEHRP class D sites) . . . . 58

4.5 Stations to calculate site amplification (NEHRP class E sites) . . . 61

4.6 Sample shear wave velocity structure . . . . . . . . . . . . . 64

5.1 Input parameters for simulations $\ldots \ldots \ldots$. . . . . . . . . 89

5.2 Comparison of the model bias with different stress drop and pulsing percentage ......................... 91

5.3 Linear and nonlinear correction coefficients used in this study . . . 101 


\section{LIST OF FIGURES}

2.1 Gaussian noise . . . . . . . . . . . . . . 15

2.2 Saragoni-Hart taper window . . . . . . . . . . . . 16

2.3 Tapered Gaussian noise . . . . . . . . . . . . . . . 17

2.4 Fourier spectrum of the noise f. . . . . . . . . . 18

2.5 Brune spectrum . . . . . . . . . . . . . . . . . . 19

2.6 Simulated Fourier spectrum . . . . . . . . . . . . 20

2.7 Simulated seismogram $\ldots \ldots \ldots \ldots . \ldots . \ldots . \ldots 21$

2.8 Comparison of corner frequency . . . . . . . . . . . . . 22

2.9 The shape of source spectra and the effects of the path term and kappa factor. . . . . . . . . . . . . . . . 23

2.10 Tectonic structure of Taiwan . . . . . . . . . . . . 24

2.11 Tectonics of the 21 September Chi-Chi Taiwan Earthquake . . . . . 25

2.12 Destructive earthquakes in Taiwan(1900-1999) . . . . . . . . 26

2.13 Generalized geological map of Taiwan . . . . . . . . . . . . . 27

3.1 Flow chart for earthquake data processing . . . . . . . . . . . . 42

3.2 Stations used in this study . . . . . . . . . . . . . . 43

3.3 Sample seismogram . . . . . . . . . . . . . . . . . . . 44

3.4 Commonly used taper functions for data processing . . . . . . . . 45

4.1 The fault plane adopted in this study . . . . . . . . . . 68

4.2 The slip distribution model used in this study . . . . . . . . . 69

ix 
4.3 Sample Fourier acceleration amplitude . . . . . . . . . . . . . 70

$4.4 \kappa_{0}$ for rock stations $(\mathrm{U}-\mathrm{D}) \ldots \ldots \ldots \ldots \ldots \ldots$

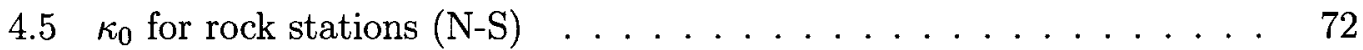

$4.6 \kappa_{0}$ for rock stations $(\mathrm{E}-\mathrm{W}) \ldots \ldots \ldots \ldots \ldots$

$4.7 \kappa_{0}$ for soil stations $(\mathrm{U}-\mathrm{D}) \ldots \ldots \ldots \ldots \ldots$

$4.8 \kappa_{0}$ for soil stations $(\mathrm{N}-\mathrm{S}) \ldots \ldots \ldots \ldots \ldots$

$4.9 \kappa_{0}$ for soil stations $(\mathrm{E}-\mathrm{W}) \ldots \ldots \ldots \ldots \ldots$

4.10 (a) Attenuation curve of PGA for rock stations (vertical) (b) Residual distribution of PGA attenuation regression analysis for rock stations (vertical) . . . . . . . . . . . . . . .

4.11 (a) Attenuation curve for Fourier acceleration amplitude at $1 \mathrm{~Hz}$ (vertical). (b) Residual distribution of attenuation analysis of Fourier acceleration amplitude at $1 \mathrm{~Hz}$ (vertical) $\ldots \ldots \ldots \ldots$

4.12 (a) Attenuation curve for Fourier acceleration amplitude at $5 \mathrm{~Hz}$ (vertical). (b) Residual distribution of attenuation analysis of Fourier acceleration amplitude at $5 \mathrm{~Hz}$ (vertical) $\ldots \ldots \ldots 79$

$4.13 \mathrm{Q}$ model; the vertical error bars are $95 \%$ confidence interval . . . . . 80

4.14 The shear wave velocity $\left(V_{s}\right)$ structure at station CHY006 . . . . . 81

4.15 The shear wave velocity $\left(V_{s}\right)$ structure at station CHY001 . . . . 82

4.16 Comparison of site amplifications (NEHRP class C) . . . . . . . 83

4.17 Comparison of site amplifications (NEHRP class D) . . . . . . . 84

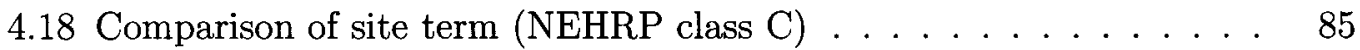

4.19 Comparison of site term (NEHRP class D) . . . . . . . 86

4.20 Duration of motions in the Chi-Chi mainshock . . . . . . . . . 87

5.1 Model bias comparison for models with different stress drop (20-100 bars) but fixed pulsing percentage $(50 \%) \ldots \ldots . \ldots 102$

5.2 Model bias for the preferred model on rock stations (NEHRP class C) 103 
5.3 Model bias versus distance for the preferred model (NEHRP class C) at $0.5 \mathrm{~Hz} \ldots \ldots \ldots \ldots \ldots$. . . . . . . . . . . . . . . 104

5.4 Model bias versus distance for the preferred model (NEHRP class C) at $1 \mathrm{~Hz} \ldots \ldots \ldots \ldots \ldots \ldots \ldots$

5.5 Model bias versus distance for the preferred model (NEHRP class C) at $5 \mathrm{~Hz} \ldots \ldots \ldots \ldots \ldots \ldots \ldots$

5.6 Model bias versus distance for the preferred model (NEHRP class C) at $10 \mathrm{~Hz} \ldots \ldots \ldots \ldots \ldots \ldots \ldots$

5.7 Model bias for the preferred model on rock stations (NEHRP class C) using pre-determined slip model . . . . . . . . . . . . . 106

5.8 Model bias versus distance for the pre-determined slip model (NEHRP class $\mathrm{C})$ at $0.5 \mathrm{~Hz} \ldots \ldots \ldots \ldots$. . . . . . . . . . . 107

5.9 Model bias versus distance for the pre-determined slip model (NEHRP class C) at $1 \mathrm{~Hz} \ldots \ldots \ldots \ldots \ldots$. . . . . . . . . . . . 107

5.10 Model bias versus distance for the pre-determined slip model (NEHRP class C) at $5 \mathrm{~Hz} \ldots \ldots \ldots \ldots \ldots$. . . . . . . . . . . 108

5.11 Model bias versus distance for the pre-determined slip model (NEHRP class C) at $10 \mathrm{~Hz} \ldots \ldots \ldots \ldots . \ldots \ldots$

5.12 Map view of the model bias for the preferred model (NEHRP class C) with random $\operatorname{slip}(1 \mathrm{~Hz})$ and pre-determined $\operatorname{slip}(1 \mathrm{~Hz}) \ldots \ldots . . . .109$

5.13 Map view of the model bias for the preferred model (NEHRP class C) with random $\operatorname{slip}(10 \mathrm{~Hz})$ and pre-determined $\operatorname{sip}(10 \mathrm{~Hz}) \ldots \ldots$

5.14 Comparison of the model bias with different site amplification functions 111

5.15 Model bias versus distance for the preferred model (NEHRP class C) using $\mathrm{H} / \mathrm{V}$ as site amplification $(1 \mathrm{~Hz}) \ldots \ldots \ldots 112$

5.16 Model bias versus distance for the preferred model (NEHRP class C) using $\mathrm{H} / \mathrm{V}$ as site amplification $(5 \mathrm{~Hz}) \ldots \ldots \ldots 112$

5.17 Model bias versus frequency for soil stations (NEHRP class D sites) with quarter wavelength approach . . . . . . . . . . . 113 
5.18 Model bias versus distance for soil stations (NEHRP class D sites) with quarter wavelength approach $(0.5 \mathrm{~Hz}) \ldots \ldots \ldots . \ldots . . \ldots 114$

5.19 Model bias versus distance for soil stations (NEHRP class D sites) with quarter wavelength approach $(2 \mathrm{~Hz}) \ldots \ldots \ldots \ldots . . \ldots 114$

5.20 Model bias versus frequency for soil stations (NEHRP class D sites) with $\mathrm{H} / \mathrm{V} \ldots \ldots \ldots \ldots \ldots \ldots \ldots \ldots$

5.21 Model bias versus distance for soil stations (NEHRP class D sites) with $\mathrm{H} / \mathrm{V}(0.5 \mathrm{~Hz}) \ldots \ldots \ldots \ldots \ldots \ldots \ldots$

5.22 Model bias versus distance for soil stations (NEHRP class D sites) with $\mathrm{H} / \mathrm{V}(2 \mathrm{~Hz}) \ldots \ldots \ldots \ldots \ldots \ldots \ldots \ldots$

5.23 Comparison of the linear correction coefficient function . . . . . . 117

5.24 Comparison of linear and nonlinear correction term for $\mathrm{PGA}=0.05 \mathrm{~g}$ based on Boore and Atkinson $(2005)(5 \mathrm{~Hz}) \ldots \ldots \ldots \ldots$

5.25 Comparison of linear and nonlinear correction term for $\mathrm{PGA}=0.15 \mathrm{~g}$ based on Boore and Atkinson (2005) $(5 \mathrm{~Hz}) \ldots \ldots \ldots \ldots$

5.26 Comparison of the exponential form of the linear and nonlinear correction term for PGA $=0.15 \mathrm{~g}$ based on Boore and Atkinson $(2005)(5 \mathrm{~Hz})$

5.27 Model bias versus frequency for soil stations (NEHRP class D sites) with linear correction by Boore and Atkinson (2005) . . . . . . . . . 120

5.28 Model bias versus distance for soil stations (NEHRP class D sites) after linear correction $(0.5 \mathrm{~Hz})$ using Boore and Atkinson (2005) method .

5.29 Model bias versus distance for soil stations (NEHRP class D sites) after linear correction $(2 \mathrm{~Hz})$ using Boore and Atkinson (2005) method . .

5.30 Model bias versus distance for soil stations (NEHRP class D sites) after linear correction (5 Hz) using Boore and Atkinson (2005) method .

5.31 Model bias versus distance for soil stations (NEHRP class D sites) after linear correction $(10 \mathrm{~Hz})$ using Boore and Atkinson (2005) method . .

5.32 Model bias versus frequency for soil stations (NEHRP class D sites) with nonlinear correction by Boore and Atkinson (2005) . . . . . . 125 
5.33 Model bias versus distance for soil stations (NEHRP class D sites) after linear and nonlinear correction $(0.5 \mathrm{~Hz})$ using Boore and Atkinson (2005) method .

5.34 Model bias versus distance for soil stations (NEHRP class D sites) after linear and nonlinear correction $(2 \mathrm{~Hz})$ using Boore and Atkinson (2005) method . . . . . . . . . . . . . . . . . .

5.35 Model bias versus distance for soil stations (NEHRP class D sites) after linear and nonlinear correction $(5 \mathrm{~Hz})$ using Boore and Atkinson (2005) method . . . . . . . . . . . . . . . . .

5.36 Model bias versus distance for soil stations (NEHRP class D sites) after linear and nonlinear correction $(10 \mathrm{~Hz})$ using Boore and Atkinson (2005) $\operatorname{method} \ldots \ldots \ldots \ldots \ldots \ldots$

5.37 Topographic map of Taipei region . . . . . . . . . . . . . . . . 130

5.38 Model bias versus frequency for soil stations (NEHRP class D sites) in the Taipei basin . . . . . . . . . . . . . . . . . . . . 131

5.39 Model bias versus frequency for soil stations (NEHRP class D sites) in the Taipei basin (Net basin effect) . . . . . . . . . . . . . . . 131

5.40 Model bias versus distance for soil stations (NEHRP class D sites) in

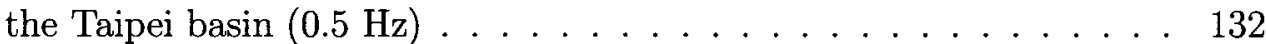

5.41 Model bias versus distance for soil stations (NEHRP class D sites) in the Taipei basin $(1 \mathrm{~Hz}) \ldots \ldots \ldots \ldots$

5.42 Model bias versus distance for soil stations (NEHRP class D sites) in the Taipei basin $(10 \mathrm{~Hz}) \ldots \ldots \ldots \ldots$. . . . . . . . . 134

A.1 Sample simulated accelerogram at station ALS . . . . . . . . . . . . . 144

A.2 Sample simulated accelerogram at station HWA056 . . . . . . . 145 


\section{LIST OF ACRONYMS}

This section contains the definition of the acronyms used in the manuscript.

PGA: Peak Ground Acceleration

PGV: Peak Ground Velocity

Faccn: Fourier Acceleration Amplitude

PSA: Pseudo Spectrum Acceleration (5\% damping)

NEHRP: National Earthquake Hazard Reduction Program

USGS: United States Geological Survey

CWB: Central Weather Bureau (Taiwan)

TSMIP: Taiwan Strong-Motion Instrumentation Program

SMSIM: Stochastic Model SIMulation

FINSIM: FINite fault stochastic SIMulation

EXSIM: EXtended fault stochastic SIMulation

FFT: Fast Fourier Transformation

IFFT: Inverse Fast Fourier Transformation

$M_{W}$ : Moment Magnitude

E-W: East-North direction

N-S: North-South direction

U-D: Vertical direction

NCREE: National Center for Research on Earthquake Engineering (Taiwan)

H/V: Horizontal to Vertical ratio of the Fourier acceleration amplitudes

xiv 


\section{Chapter 1}

\section{INTRODUCTION}

\subsection{Purpose of Study}

The September 21, 1999, Chi-Chi earthquake $\left(M_{W}=7.6\right)$ is one of the largest earthquakes to strike Taiwan this century. During this earthquake, many strong motion records were collected, greatly increasing the world's near-fault strong motion database. The Chi-Chi database, especially the rich near source records, offers us a good opportunity to investigate the earthquake's source parameters, the path and the site amplification effects, including the non-linear soil response.

In engineering, the peak value of ground acceleration or velocity (PGA or PGV) is often used to conduct some preliminary analysis. However, the PGA or PGV of the shaking cannot adequately characterize the ground motion, since the duration and the frequency content of the shaking are the important factors that control the earthquake damage. An earthquake with low peak ground motion amplitude but longer duration may release more energy than the one with large peak value but shorter duration. The response spectrum of the shaking, describing the response of a single-degree-of-freedom to the motion as a function of natural frequency, is a useful description as it conveys information on the frequency content of the ground motion, and is somewhat influenced by the duration.

According to Taiwan's Interior Department records, the death toll in the ChiChi earthquake was 2,470 and 11,305 people were injured (Shin and Teng, 2001). 
Thousands of houses collapsed making more than 100,000 people homeless. Among several factors leading to the high casualty rate in the Chi-Chi earthquake, buildings collapsing in the metropolitan area is a key one. Underestimation of seismic hazard in the building code at least partly explains these collapses. Understanding the source characterization of the earthquake, the propagation properties of the crust in Taiwan Island and the site amplification behavior may help us to improve the building code for this region.

In this study, I use the extended fault stochastic simulation method developed by Motazedian (2002) and Motazedian and Atkinson (2005) to model ground motions of the $M_{W}=7.6$ Chi-Chi main shock. By calibrating the simulation parameters to reproduce the ground motion, we can determine the contribution of the source, propagation path and site parameters to the ground motion. Furthermore, by analyzing the simulated results, we may determine the slip distribution model, the directivity effect, the basin effect and the non-linear response of the soil stations. Better understanding of these effects improves our ability to predict ground motion for future large earthquakes, hence improve building codes.

\subsection{Organization of Work}

This study focuses on the source characterization of the 1999 Chi-Chi earthquake, seismic wave path parameters of Taiwan Island and site amplification of the strong motion stations. These effects are discussed in six chapters. In Chapter 1,

the purpose of the project and the outline of the tasks is presented. Chapter 2 overviews and compares the theoretical background of the point source stochastic simulation technique (SMSIM (Boore, 1996)), the finite fault stochastic simulation method (FINSIM (Beresnev and Atkinson, 1998a)) and the extended fault stochastic 
simulation approach (EXSIM (Motazedian and Atkinson, 2005)), which is adopted in this study. Chapter 3 describes the strong motion seismic network in Taiwan, the available dataset used in this study and the procedures of the data processing. Chapter 4 presents taken steps to obtain the source parameters (fault geometry, earthquake location and ground motion duration), the path (geometric attenuation spreading and $\mathrm{Q}$ model), and the site effects (site amplification function and the kappa factor). Chapter 5 discusses the input parameters for ground motion simulation at rock stations, the process of model calibration, the parameters determined by the simulation and the simulation results for both rock and soil stations. Chapter 6 presents the conclusions.

\subsection{Contribution}

The author, under the supervision of his advisors, has made the following contributions in this study:

1. Data processing of 441 strong motion three-component recordings of the ChiChi mainshock.

2. Characterizing the seismic source and path parameters of the 1999 Chi-Chi earthquake, including calculating the kappa value for all of the stations (two horizontal and vertical components), and the regression of ground motions to determine the geometric spreading attenuation and Q model for the main shock.

3. Calculating the site amplification function for rock and soil stations based on the shear wave velocity information provided by National Center for Research on Earthquake Engineering of Taiwan. 
4. Calculating $\mathrm{H} / \mathrm{V}$ ratio and evaluating its accuracy as a site amplification function.

5. Determining stress drop and pulsing percentage (two free source parameters), based on extended fault stochastic simulation techniques.

6. Analyzing the nonlinear soil response by applying the calibrated model parameters (from NEHRP class C stations) to the soil stations (NEHRP class D sites) using the empirical correction function.

7. Analyzing the basin effect of the soil stations in the Taipei basin area. 


\section{Chapter 2}

\section{LITERATURE SURVEY}

\subsection{Overview}

The main modeling technique used in this study is the stochastic model, which is reviewed here. In stochastic simulation of ground motions, a Gaussian noise signal with zero mean and unit variance is first generated in the time domain as shown in Figure 2.1. Then, a window such as the Saragoni-Hart taper window (Figure 2.2) with a specified duration time is applied to the noise signal. Figure 2.3 shows the tapered Gaussian noise. Then, the tapered noise signal is transformed by FFT (Fast Fourier Transformation) from the time domain to the frequency domain to obtain the Fourier amplitude acceleration spectrum of the noise signal (Figure 2.4). To model a specific earthquake magnitude at a given distance, the Brune model spectrum (Figure 2.5) is often calculated (Equation 2.10) and multiplied with the spectrum of the noise signal (Boore, 1983). Figure 2.6 is the spectrum of a specified earthquake at a target distance based on the stochastic simulation method. Finally, the simulated spectrum is transformed back to the time domain by IFFT (Inverse Fast Fourier Transformation) to get the seismogram, as shown in Figure 2.7. When using the above procedure to generate the simulated seismogram, the parameters chosen to specify the Brune spectrum model as a function of magnitude and distance are the key factors. In this study, most of the efforts are based on how to adjust parameters to specify the seismological model, using regional empirical parameters and regression 
analysis of the recorded strong motion data. In the following section, the theoretical background of the stochastic simulation technique is presented in more detail. Its application to the Chi-Chi earthquake is then introduced.

\subsection{Stochastic Simulation}

\subsubsection{Point Source Stochastic Simulation}

The stochastic simulation method is a simple, powerful and widely used technique to predict ground motions (Boore, 1983; Boore and Atkinson, 1987; Atkinson and Boore, 1997; Beresnev and Atkinson, 1997, 1998a, 1999; Atkinson and Silva, 2000; Castro et al., 2001; Roumelioti and Kiratzi, 2002; Roumelioti and Beresnev, 2003). It is particularly useful to simulate the higher-frequency ground motion which is of interest to most engineers $(f>0.2 \mathrm{~Hz})$ (Boore, 2003). This method assumes that higher-frequency earthquake ground motions can be represented as a band-limited Gaussian noise which has an $\omega^{2}$ mean spectrum (Beresnev and Atkinson, 1998b), sometimes referred to as a Brune spectrum (Brune, 1970). Various factors affecting ground motions $\left(F A\left(M_{0}, R, f\right)\right)$ can be simply expressed as Equation 2.1, which is the product of the source term $\left(E\left(M_{0}, f\right)\right)$, the path term $(P(R, f))$ and the site term $(G(f))$, in the frequency domain (Boore, 2003):

$$
F A\left(M_{0}, R, f\right)=E\left(M_{0}, f\right) P(R, f) G(f)
$$

where $f$ is frequency in Hertz, $M_{0}$ is the seismic moment introduced by Aki (1966) and $R$ is the hypocentral distance.

The spectrum of ground motions at a site is the combined effects of the source, the path and the site. In the following sections, these factors will be discussed in 
more detail.

The source effects In the stochastic simulation technique, the most frequently used earthquake source model is the $\omega$-square shape spectrum (Aki, 1967; Brune, 1970). Apart from the widely used $\omega$-square model, there are many other models used in stochastic simulation (Boatwright and Choy, 1992; Atkinson and Boore, 1995; Frankel et al., 1996; Haddon, 1996; Atkinson and Silva, 2000). The earthquake source spectra for all of the models can be written in a general form as Equation 2.2.

$$
E\left(M_{0}, f\right)=C M_{0} S\left(f, f_{0}\right)
$$

where $C, f_{0}$ and $M_{0}$ are a constant defined in Equation 2.3, corner frequency and seismic moment, respectively. $S\left(f, f_{0}\right)$ is one of the above-mentioned spectrum models.

$$
C=\frac{R_{\theta \phi} \cdot F S \cdot P R T I T N}{4 \pi \rho \beta^{3}}
$$

where $R_{\theta \phi}$ is the radiation pattern, $F S$ is the amplification due to the free surface, PRTITN is the reduction factor that accounts for the partitioning of energy into two horizontal components $(1 / \sqrt{2}), \rho$ and $\beta$ are the density and shear wave velocity, respectively (Boore, 1983).

Among source spectra models used in the stochastic simulation, the $\omega^{2}$ model $\left(S\left(f, f_{0}\right)\right)$ introduced by Aki (1967) and Brune (1970) is used in this study to characterize the spectrum of subfaults that make up an extended fault plane. Equation 2.4 is the functional form of the Brune source spectrum model (Brune, 1970).

$$
S\left(f, f_{0}\right)=\frac{f^{2}}{1+\left(f / f_{0}\right)^{2}}
$$


where $f_{0}$ can be calculated by Equation 2.5 (Boore, 1983):

$$
f_{0}=4.9 \times 10^{6} \beta\left(\Delta \sigma / M_{0}\right)^{1 / 3}
$$

where $\beta$ in $\mathrm{km} / \mathrm{s}, M_{o}$ in dyne-cm, $\Delta \sigma$ in bars.

The corner frequency $\left(f_{0}\right)$ is a function of seismic moment, as illustrated in Figure 2.8. Assuming self-similarity in the source, it can be shown that $M_{0} f_{0}^{3}=$ constant (Aki, 1967; Boore, 2003).

The path effects The simplest path effects function, $P(R, f)$ (Equation 2.6), is the product of the geometrical spreading function $Z(R)$ and the anelastic attenuation which is a function of crustal quality factor, Q, (Boore, 2003):

$$
P(R, f)=Z(R) \exp [-\pi f R / Q(f) \beta]
$$

The geometrical spreading function $Z(R)$ can be assigned with various forms. The simplest form for $Z(R)$ is $Z(R)=\frac{1}{R^{n}}$. Alternatively, a hinged linear form in which $n$ varies with distance may also be used, as described by Atkinson (2004). The anelastic attenuation function is discussed in more detail in Chapter 4.

The site effects The site effects, defined as Equation 2.7, are comprised of two separate parts: site amplification $(A(f))$ and the high frequency diminution operator $(D(f))$.

$$
G(f)=A(f) D(f)
$$

The site amplification $(A(f))$ as a function of frequency can be calculated using the quarter wavelength approximation approach (Boore and Joyner, 1997). The high fre- 
quency diminution operator $(D(f))$ accounts for the path-independent loss of highfrequency in the ground motions (Boore, 2003). Some researchers (Papageorgiou and Aki, 1983) attribute $D(f)$ to source processes while others suggest it is a site effect near the recording station (Hanks, 1982). The form of $D(f)$ is typically represented as a 4th order butterworth filter (Equation 2.8), or an exponential filter (Equation 2.9) (Boore, 1983, 2003).

$$
\begin{gathered}
D(f)=\left[1+\left(f / f_{\max }\right)^{8}\right]^{-1 / 2} \\
D(f)=\exp \left(-\pi \kappa_{0} f\right)
\end{gathered}
$$

where $f_{\max }$ is the high-cut frequency introduced by Hanks (1982), and $\kappa_{0}$ is the "kappa" parameter introduced by Anderson and Hough (1984).

After separate discussion of the source, path and site effects, in the following sections, their combined effects will be illustrated. Equation 2.10 presents the acceleration spectrum of the shear wave with seismic moment $M_{0}$ at hypocentral distance $R$ from a point source earthquake.

$$
F A\left(M_{0}, R, f\right)=C M_{0} \frac{(2 \pi f)^{2}}{1+\left(f / f_{0}\right)^{2}} \exp \left(-\pi f \kappa_{0}\right) \frac{\exp (-\pi f R / Q \beta)}{R} A(f)
$$

where $C M_{0} \frac{(2 \pi f)^{2}}{1+\left(f / f_{0}\right)^{2}}$ is the source term discussed above in Equation $2.2, \frac{\exp (-\pi f R / Q \beta)}{R}$ is the path term in which $\frac{1}{R}$ is the geometric spreading function and $\exp (-\pi f R / Q \beta)$ accounts for the anelastic attenuation effect, and $\exp \left(-\pi f \kappa_{0}\right) A(f)$ is the site term for the near surface high frequency diminution and site amplification.

Figure 2.9 presents the shape of the source term, the effects of the path term on the source term and the combined effects of the path and high-frequency diminution 
operator $\left(\exp \left(-\pi f \kappa_{0}\right)\right)$. The top line is the source spectra with a specified Brune model at distance $R$ ( $R=1$ in this example). The middle line is the product of the source term and the path term (only the anelastic attenuation affects the shape of the spectrum in this simple example). The bottom line is the product of the source term, the path term and $\exp \left(-\pi f \kappa_{0}\right)$. In this case, only the high-frequency diminution operator is included, and site amplification, $A(f)$, is not considered. It is assumed to be negligible for a very-hard rock site, as an example.

\subsubsection{Finite Fault Stochastic Simulation}

When the earthquake magnitude is small to moderate $\left(M_{W}<6\right)$, the earthquake can be treated as a point source. However, for large events $\left(M_{W}>6\right)$, the point source stochastic simulation may fail due to the effects of the rupture geometry, heterogeneity of the slip and directivity (Beresnev and Atkinson, 1998b). To overcome this limitation, Schneider et al. (1993), Beresnev and Atkinson (1998a) and Beresnev and Atkinson (1998b) extended the point source stochastic simulation technique to the finite-fault simulation. A rectangular fault plane is subdivided into several smaller subfaults. Each subfault is treated as a point source with an $\omega^{2}$ spectrum (Equation 2.2). When the earthquake is triggered at the hypocenter, the rupture propagates radially from the hypocenter along the fault plane. As the rupture propagates, each subfault is triggered as a point source. The seismic waves generated by every subfault are summed at the target recording station with proper time delay defined as Equation 2.11.

$$
t=\frac{r_{2}}{V}+\frac{r_{1}}{\beta}
$$

where $r_{1}, r_{2}, \mathrm{~V}$ and $\beta$ are the distance between the mid-point of the subfault and the recording site, the distance between the hypocenter and the subfault middle, the 
fault rupture velocity and the shear wave velocity, respectively.

According to the seismic moment conservation theory (similar to the energy conservation theory), the seismic moment of the whole fault plane $\left(M_{0}\right)$ is the sum of the seismic moments of all subfaults $\left(m_{0}\right)$. In the case of identical subfaults, the total seismic moment is given by the Equation 2.12

$$
M_{0}=N \cdot m_{0}
$$

where $N$ is the number of the subfaults.

The finite fault stochastic simulation technique was implemented in the computer program FINSIM by Beresnev and Atkinson (1998a), including fault geometry, slip distribution, stress drop, path effects and duration (Beresnev and Atkinson, 1998b). FINSIM has been extensively validated via many real recorded earthquakes, including Michoacan, Mexico (1985); Valparaiso, Chile (1985); Saguenay, Quebec (1988) and Northridge, California (1994).

\subsubsection{Refinements to the Finite Fault Stochastic Simulation}

The above finite fault stochastic simulation method calculate the corner frequency of each subfault identically using Equation 2.13

$$
f_{0 i j}=4.9 \times 10^{6} \beta\left(\Delta \sigma / M_{0 i j}\right)^{1 / 3}
$$

Where $f_{0 i j}, \Delta \sigma$ and $M_{0 i j}$ are the corner frequency, the stress drop in bars and the moment of the $i j^{\text {th }}$ subfault in dyne-cm respectively.

A conceptual drawback of FINSIM is that the simulated ground motions are dependent on the subfault size (Beresnev and Atkinson, 1998a). Motazedian (2002) 
and Motazedian and Atkinson (2005) noted that this dependency on subfault size produces different radiated energy from the entire fault using different subfault sizes. In order to eliminate these shortcomings, the dynamic corner frequency concept, in which the corner frequency is expressed as a function of time, was proposed by Motazedian (2002) and Motazedian and Atkinson (2005), and implemented in the program EXSIM (Extended Fault Simulation). The concept of pulsing percentage, in which only some parts of the fault is active at any moment in time, was also introduced in EXSIM to mimic the rupture process of real earthquakes better. In EXSIM, the corner frequency of the subfault is defined in Equation 2.14. As the rupture propagates, it activates more subfaults, which contribute to the changing frequency content of the ground motion. The corner frequency of the active subfaults decreases to lower frequency because the corner frequency is inversely proportional to the ruptured area. The value of the corner frequency is not a static variable during the motion but has a dynamic value that varies with time in the rupture process. Thus, the corner frequency of Equation 2.13 can be rewritten as:

$$
f_{0 i j}(t)=N_{R}^{-1 / 3}(t) 4.9 \times 10^{6} \beta\left(\Delta \sigma / M_{0 a v e}\right)^{1 / 3}
$$

where $f_{o i j}(t)$ becomes a function of time and controlled by $N_{R}^{-1 / 3}(t)$, which is the cumulative number of ruptured subfaults at time t. $M_{0 a v e}=M_{0} / N$ is the average seismic moment of subfaults.

The Fourier acceleration spectrum of the $i j^{\text {th }}$ subfault using the dynamic corner frequency can be written as:

$$
F A_{i j}(f)=C M_{0 i j} H_{i j}(2 \pi f)^{2} /\left[1+\left(f / f_{0 i j}(t)\right)^{2}\right]
$$


where $H_{i j}$ is a scaling factor to conserve the high-frequency spectral level of subfaults, $\mathrm{C}$ is the constant defined in Equation 2.3, $M_{0 i j}$ is the moment of the $i j^{\text {th }}$ subfault, and $f_{0 i j}(t)$ is the dynamic corner frequency of the $i j^{t h}$ subfault (Motazedian and Atkinson, 2005).

From Equation 2.15, the introduction of the dynamic corner frequency tends to decrease the level of the acceleration spectrum but this is counteracted by $H_{i j}$, keeping the total radiated energy from the subfault constant (Motazedian, 2002).

The subfaults are not always active during the rupture. When the rupture triggers a subfault, the subfault is active for a while and stops after a limited time, after which it does not contribute energy to the ground motion any more. In EXSIM, the pulsing percentage concept is used to define the percentage of active subfaults during the rupture and constrain the lower bounds of the dynamic corner frequency. The dynamic corner frequency, starting from the corner frequency of one subfault, progresses to lower values with time. When the number of activated subfaults reaches the number of subfaults defined by the pulsing percentage, it becomes a constant.

\subsection{Background of the Chi-Chi Earthquake}

\subsubsection{Tectonics and Seismicity of Taiwan}

Regional Tectonics Taiwan, located on the convergent plate boundary of the Eurasian continental plate and the Philippine oceanic plate, is a part of the panPacific seismic belt. As a result of the collision between the above mentioned two plates, a transitional zone formed along Taiwan Island. The northeastern part of Taiwan Island belongs to the Ryukyu Arc system where the Philippine plate is being subducted under the Eurasian plate. To the south of Taiwan, the Philippine plate is overriding the Eurasian plate along the Manila trench (Figure 2.10). With a $7 \mathrm{~cm} / \mathrm{yr}$. 
convergence rate in a northwest direction (Figure 2.11), Taiwan Island experiences active tectonic movements leading to frequent earthquakes.

Seismicity and Seismic hazard The seismicity of Taiwan island is very high. Within the past 100 years (1900-1999), there were at least 86 destructive earthquakes with Richter magnitude larger than 5, including 23 events above magnitude 7 (Central Weather Bureau (Taiwan), 2006). Because of this extensive seismic activity, Taiwan's 22 million people are exposed to high seismic hazard. Figure 2.12 shows its destructive earthquakes from 1900 to 1999.

\subsubsection{Geological Setting}

Figure 2.13 is a map showing the geological provinces in Taiwan. "The main island of Taiwan comprises in part of a metamorphic basement covered by Cenozoic deposits to a thickness of more than 10,000 meters. The north-south axis of deposition has shifted progressively westward with successive orogenic episodes. A considerable part of the Tertiary sediments of Taiwan have been subjected to different grades of induration or metamorphism. Large igneous intrusions are rare, but there are several important volcanic fields in northern Taiwan, eastern Taiwan, and the Taiwan Strait" (CENTRAL GEOLOGICAL SURVEY (MOEA), 2006). In this study, the rock stations include limestone, igneous or metamorphic rocks comprising hard volcanic deposits, and Micocene or older strata; the soil sites includes fluvia terraces, stiff clays and sandy gravel deposits (Roumelioti and Beresnev, 2003). 


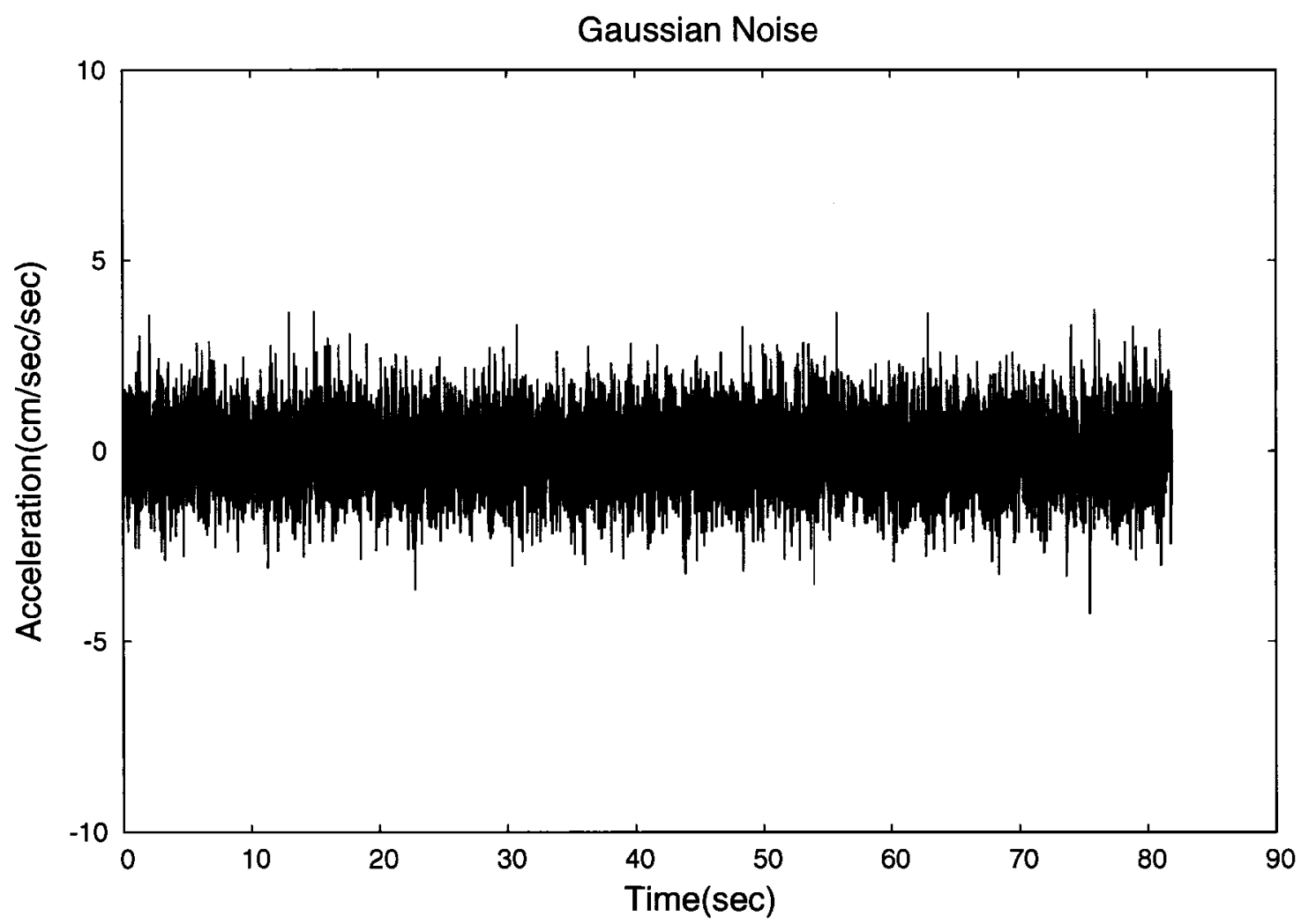

Figure 2.1. Gaussian noise (step 1 of stochastic simulation) 


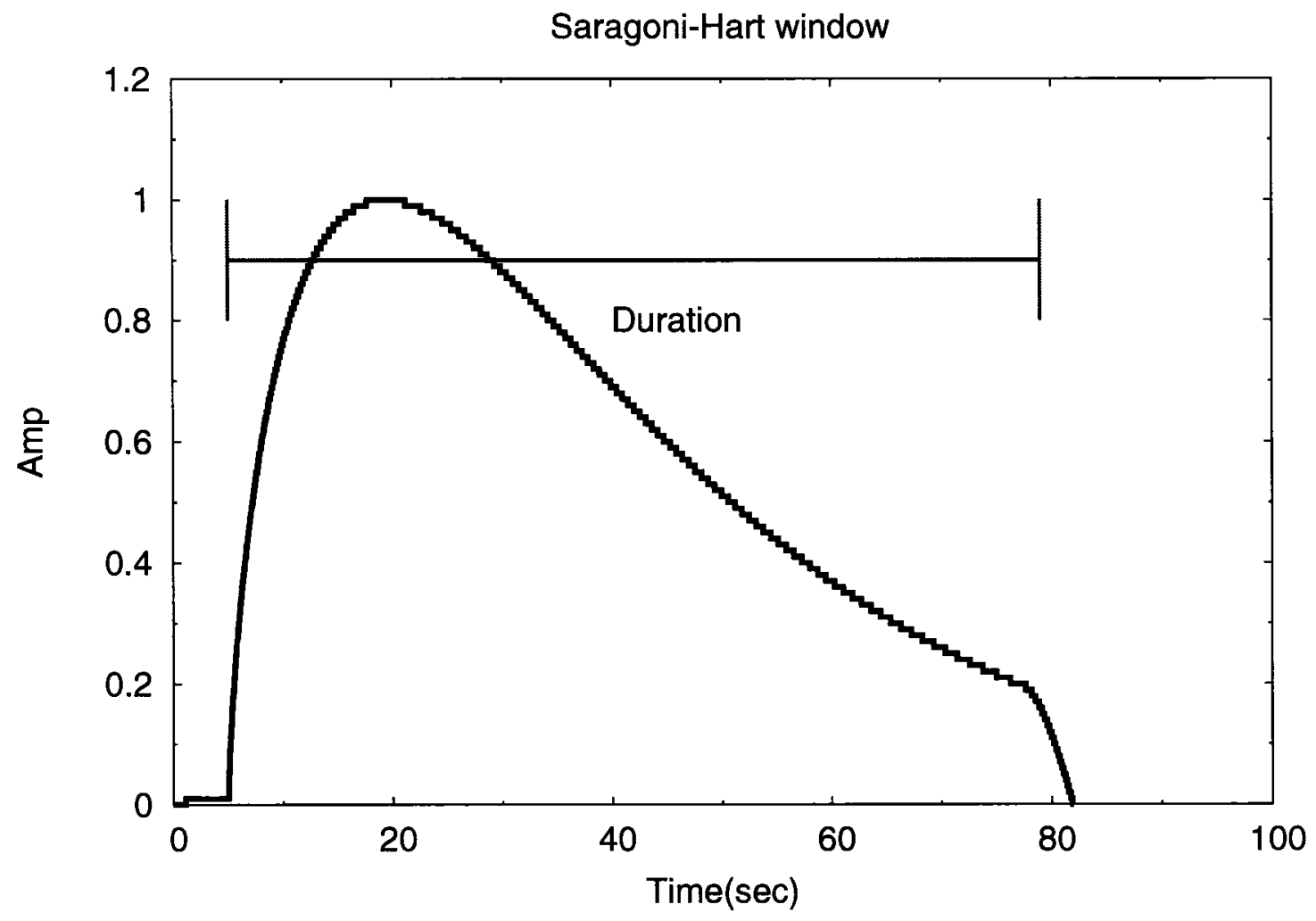

Figure 2.2. Saragoni-Hart taper window (step 2 of stochastic simulation) 


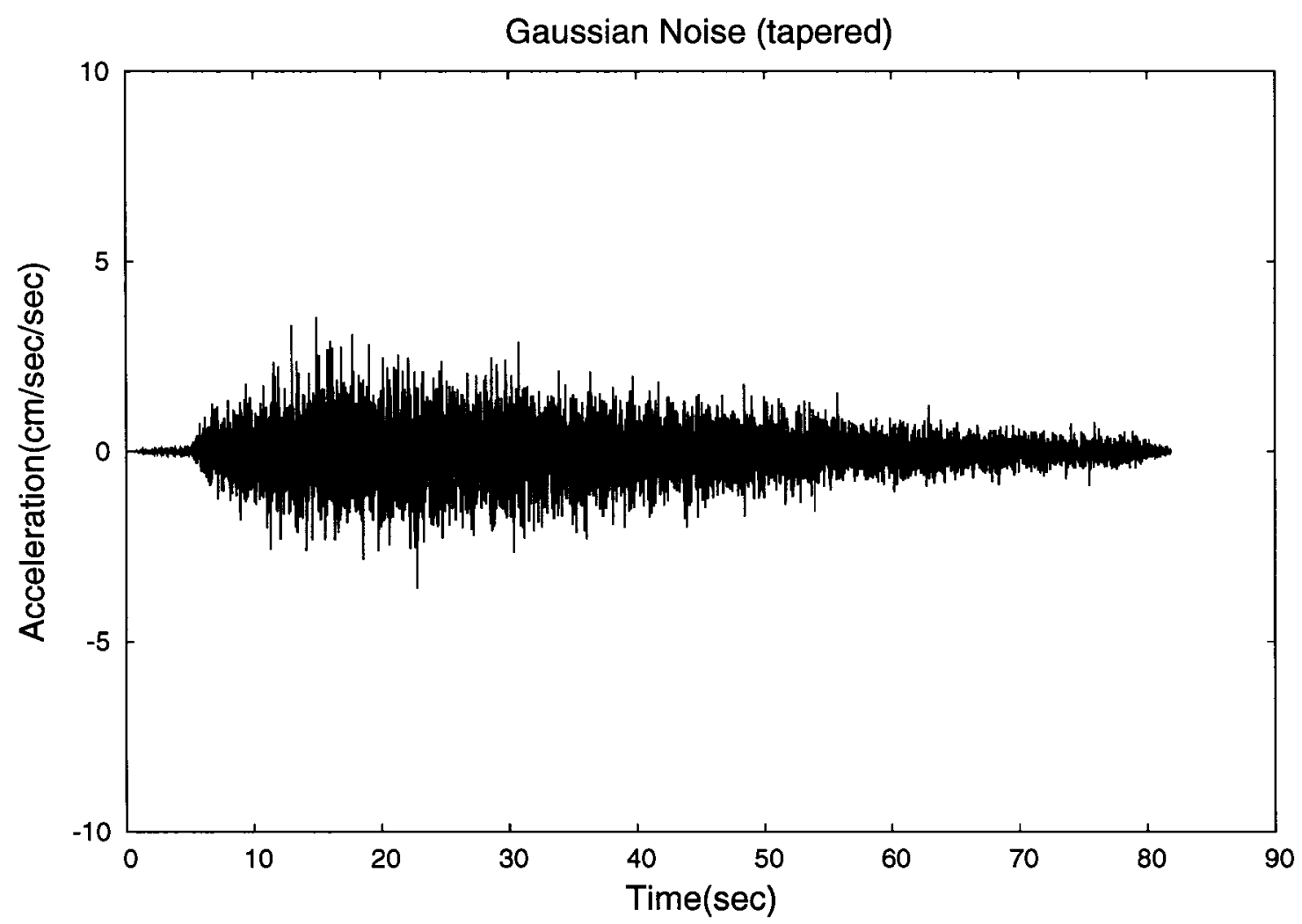

Figure 2.3. Tapered Gaussian noise (step 3 of stochastic simulation) 


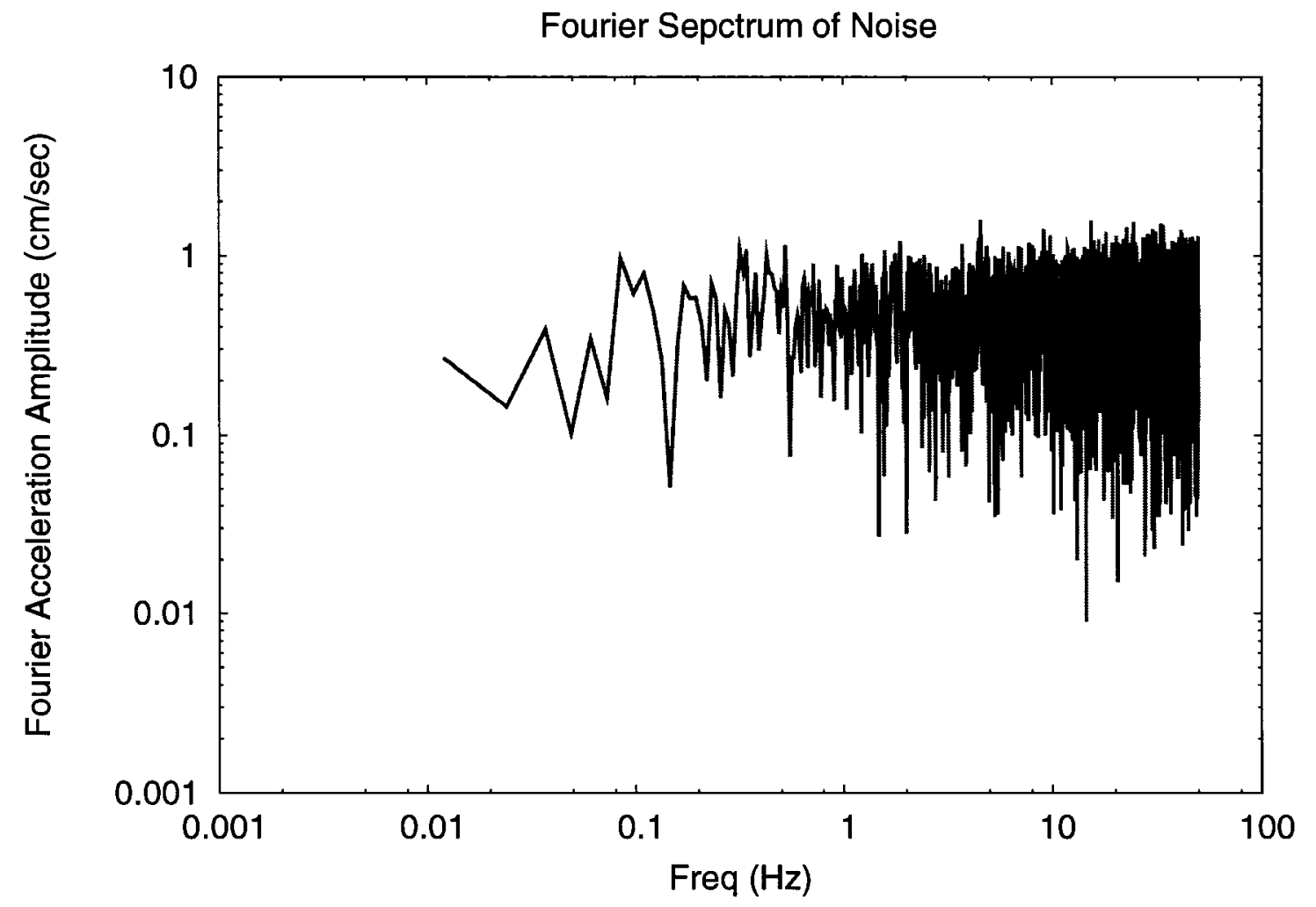

Figure 2.4. Fourier spectrum of the tapered noise (step 4 of stochastic simulation) 


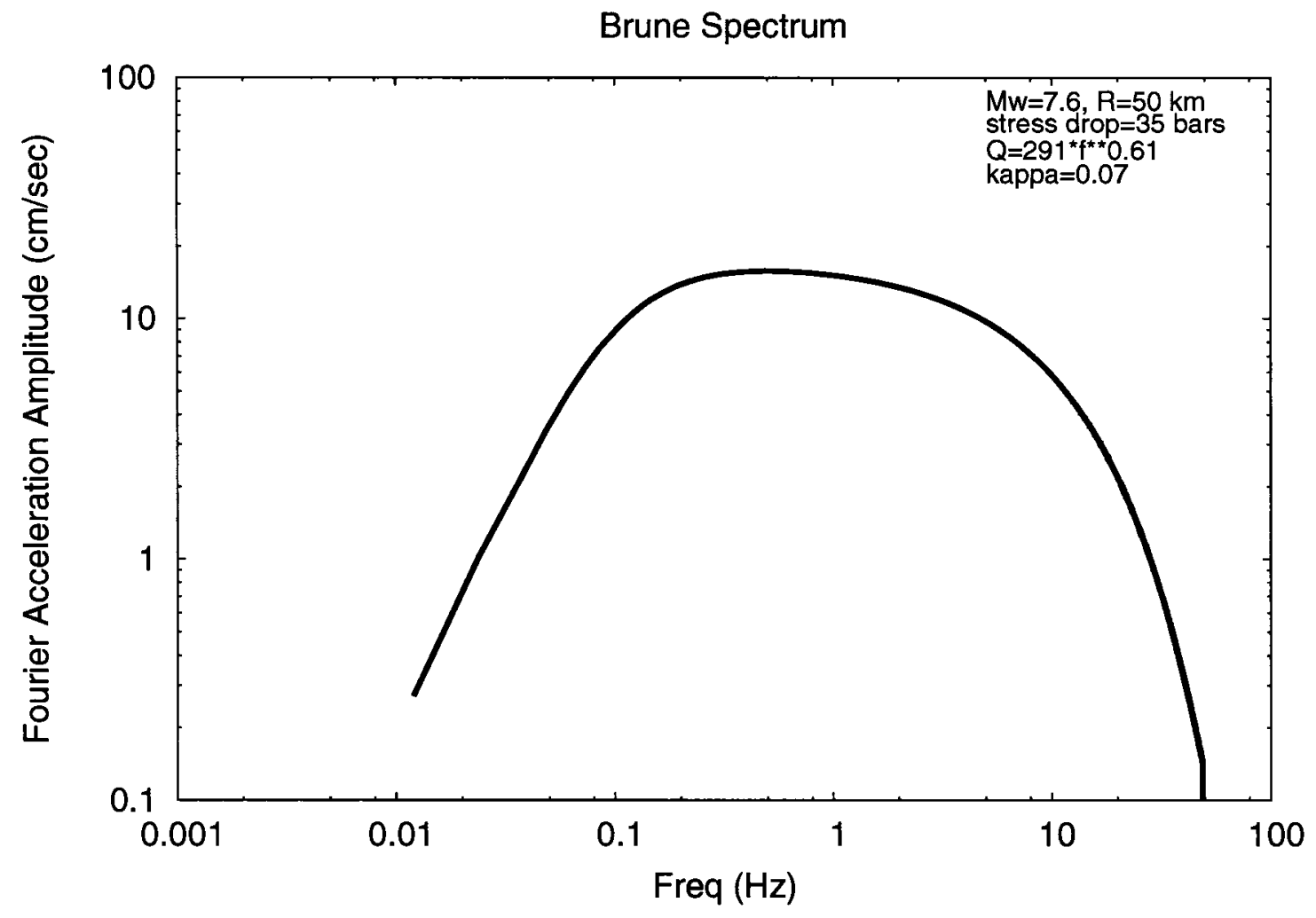

Figure 2.5. Brune source spectrum (step 5 of stochastic simulation) 
Final Fourier Spectrum(Brune $x$ Noise)

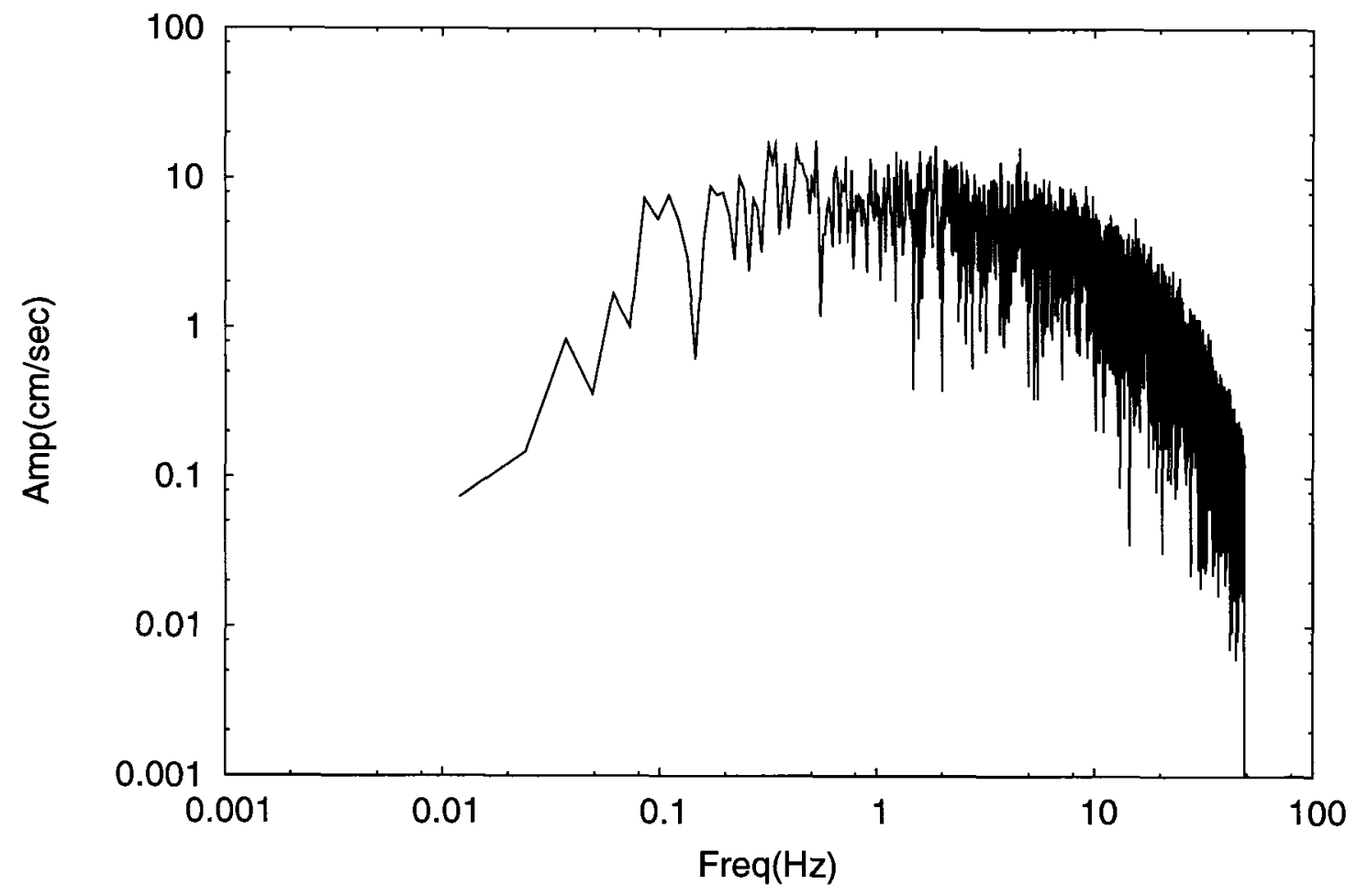

Figure 2.6. Simulated Fourier spectrum (step 6 of stochastic simulation) 


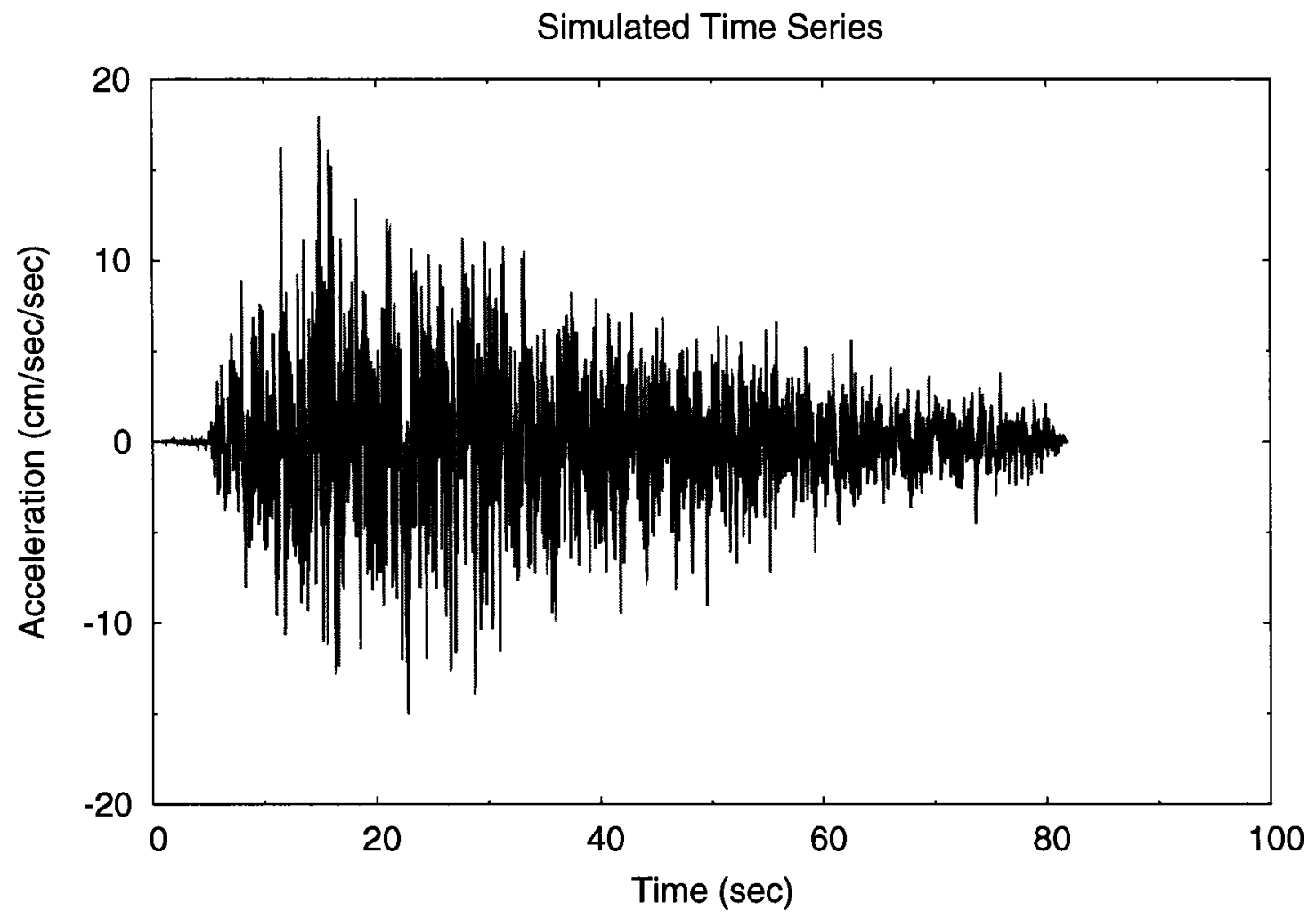

Figure 2.7. Simulated seismogram (step 7 of stochastic simulation) 


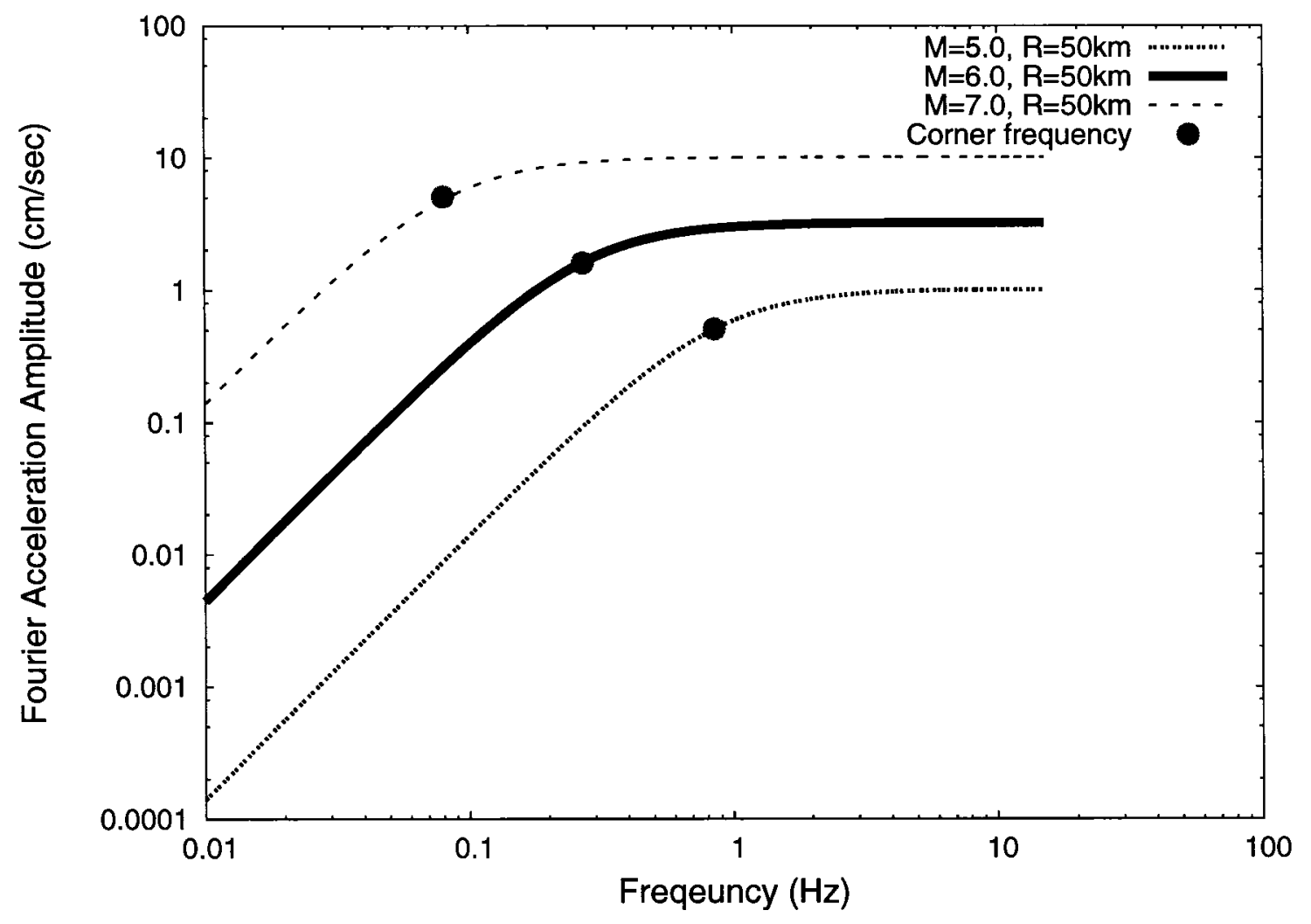

Figure 2.8. Comparison of corner frequency for Moment Magnitude 5, 6 and 7 at distance $50 \mathrm{~km}$ and stress drop $=40$ bars 


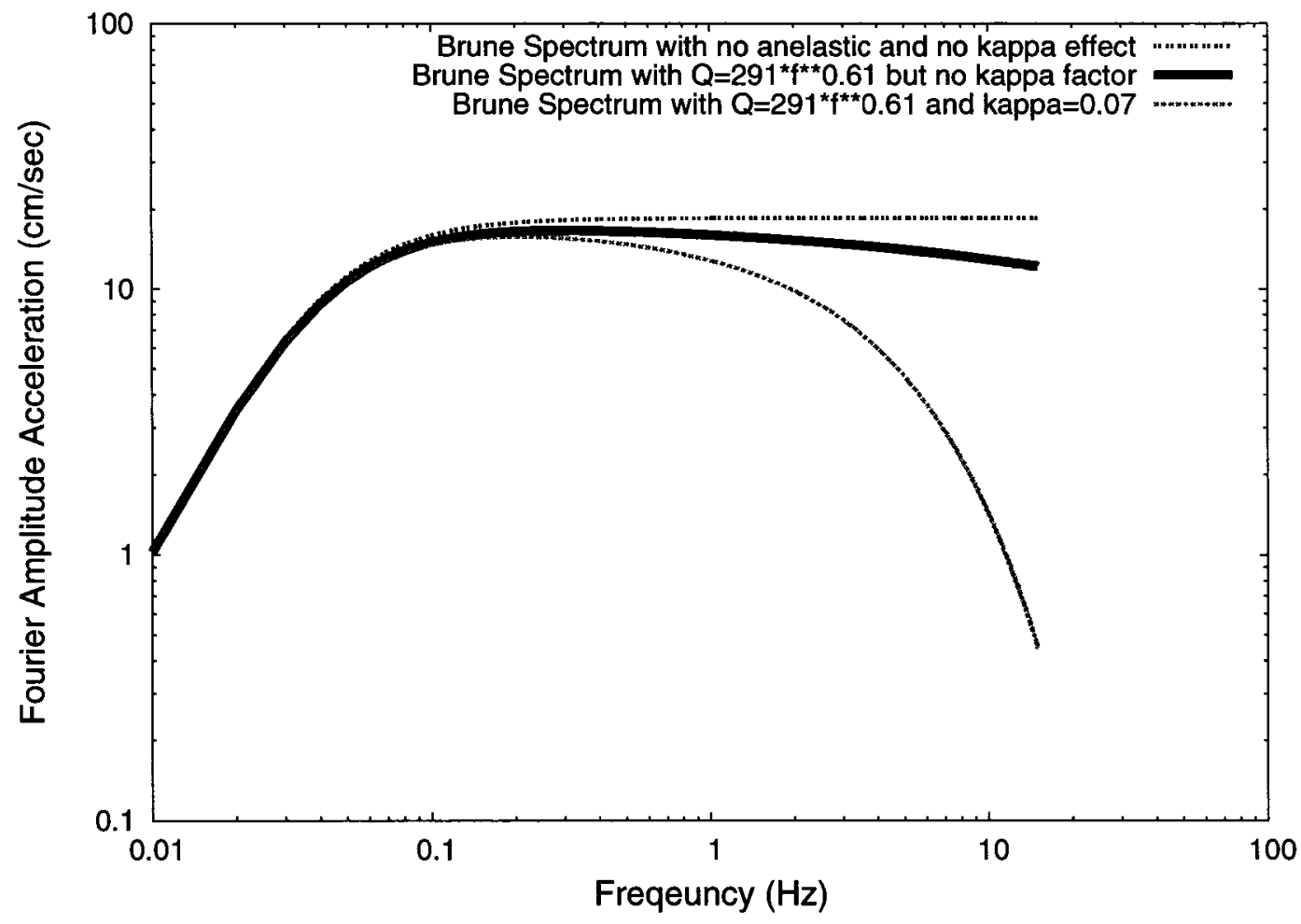

Figure 2.9. The shape of source spectra and the effects of the path term and kappa factor. 


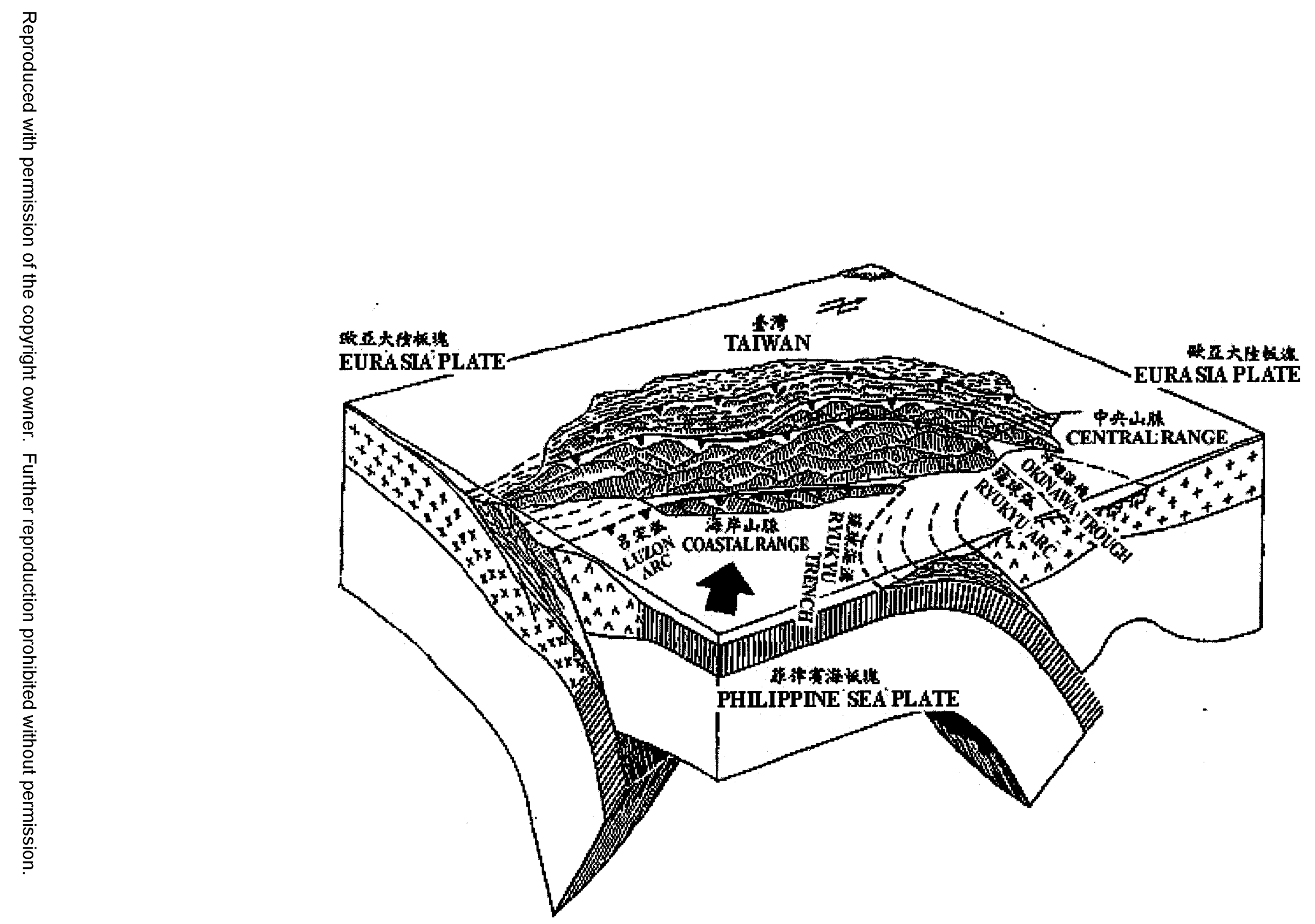

Figure 2.10. Tectonic structure of Taiwan 


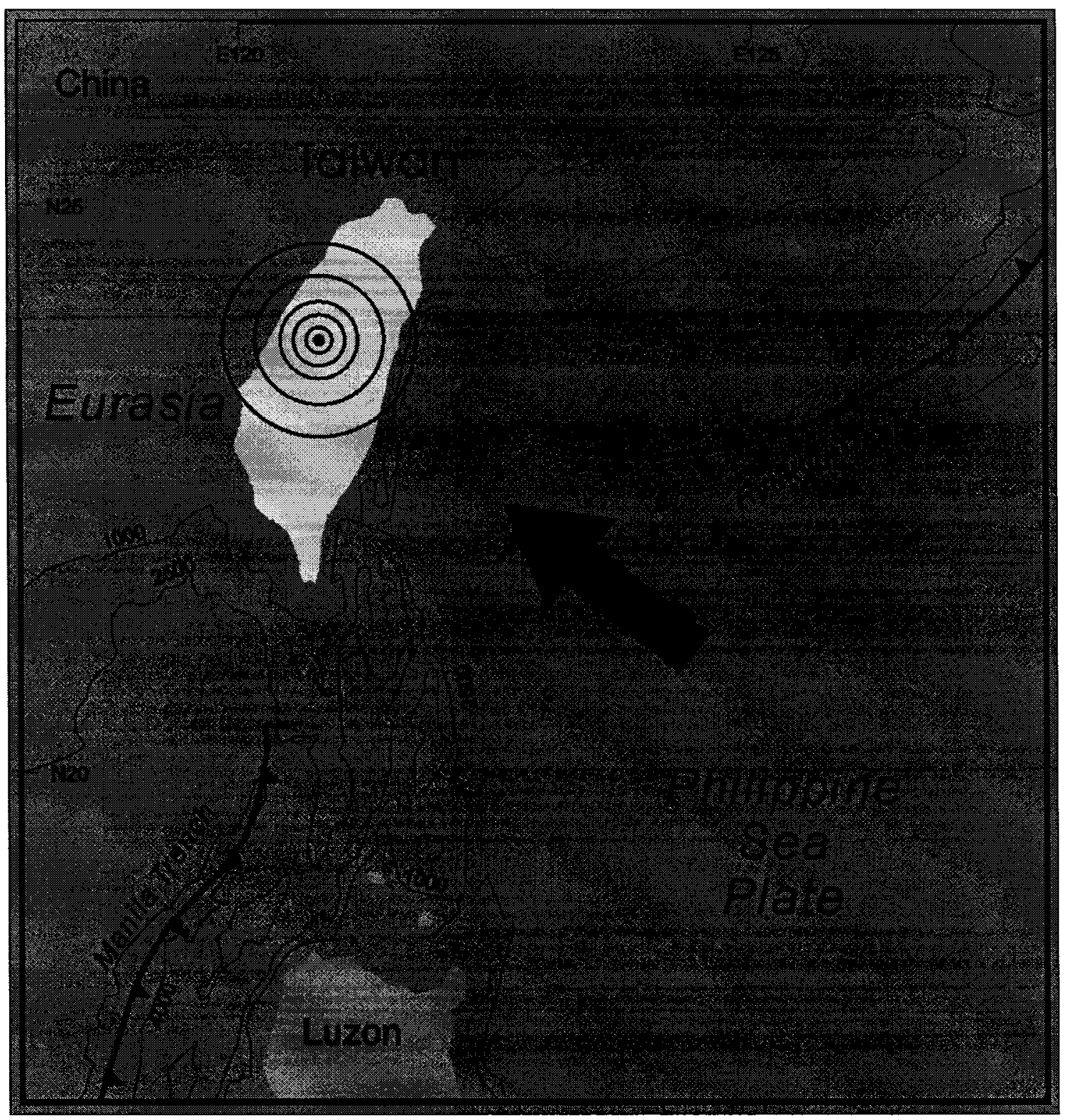

Figure 2.11. Tectonics of the 21 September Chi-Chi Taiwan Earthquake (The Institute of Earth Sciences, Academia Sinica (Taipei), 2006) 


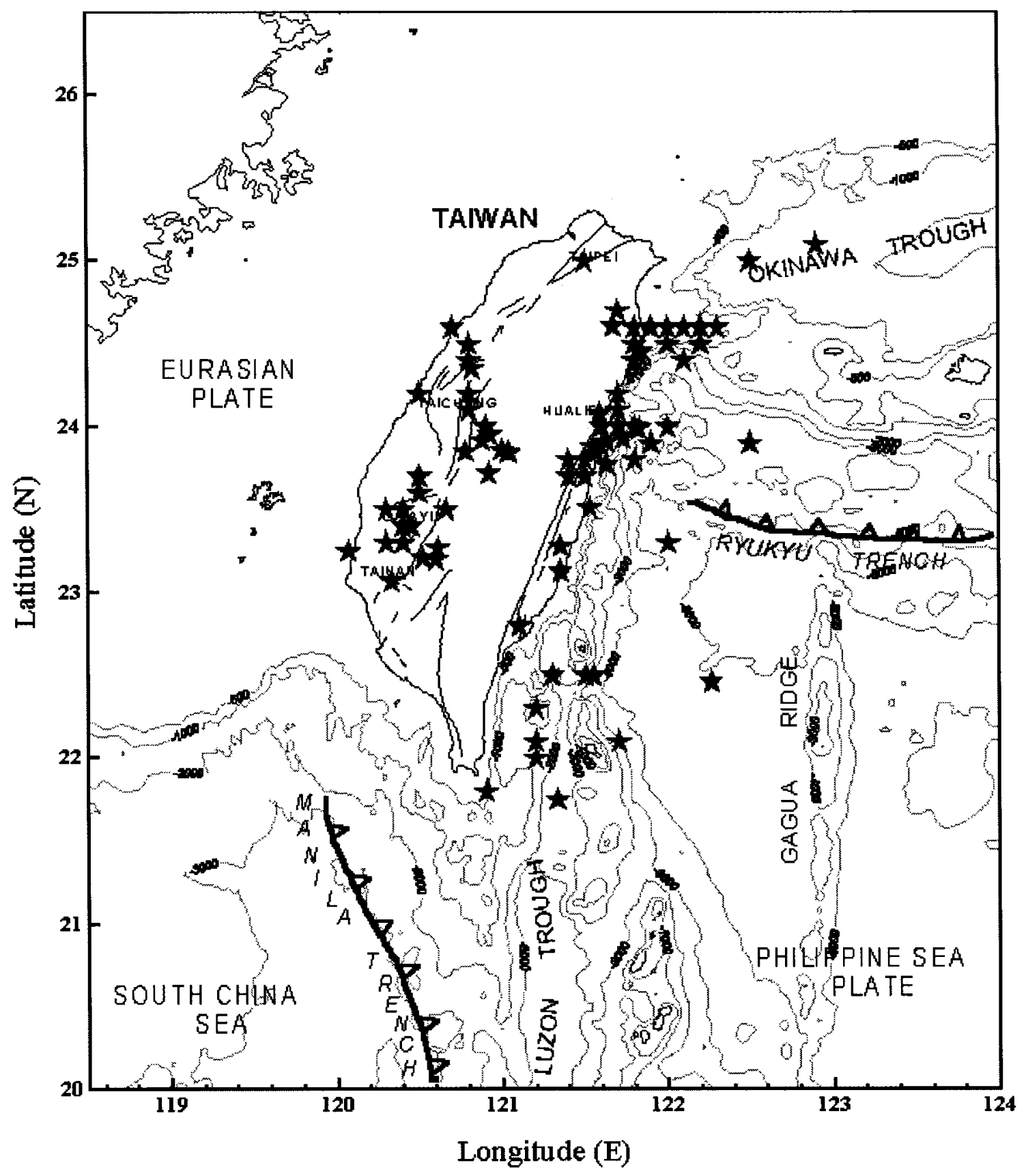

Figure 2.12. Destructive earthquakes in Taiwan (1900-1999) (Central Weather Bureau (Taiwan), 2006) 


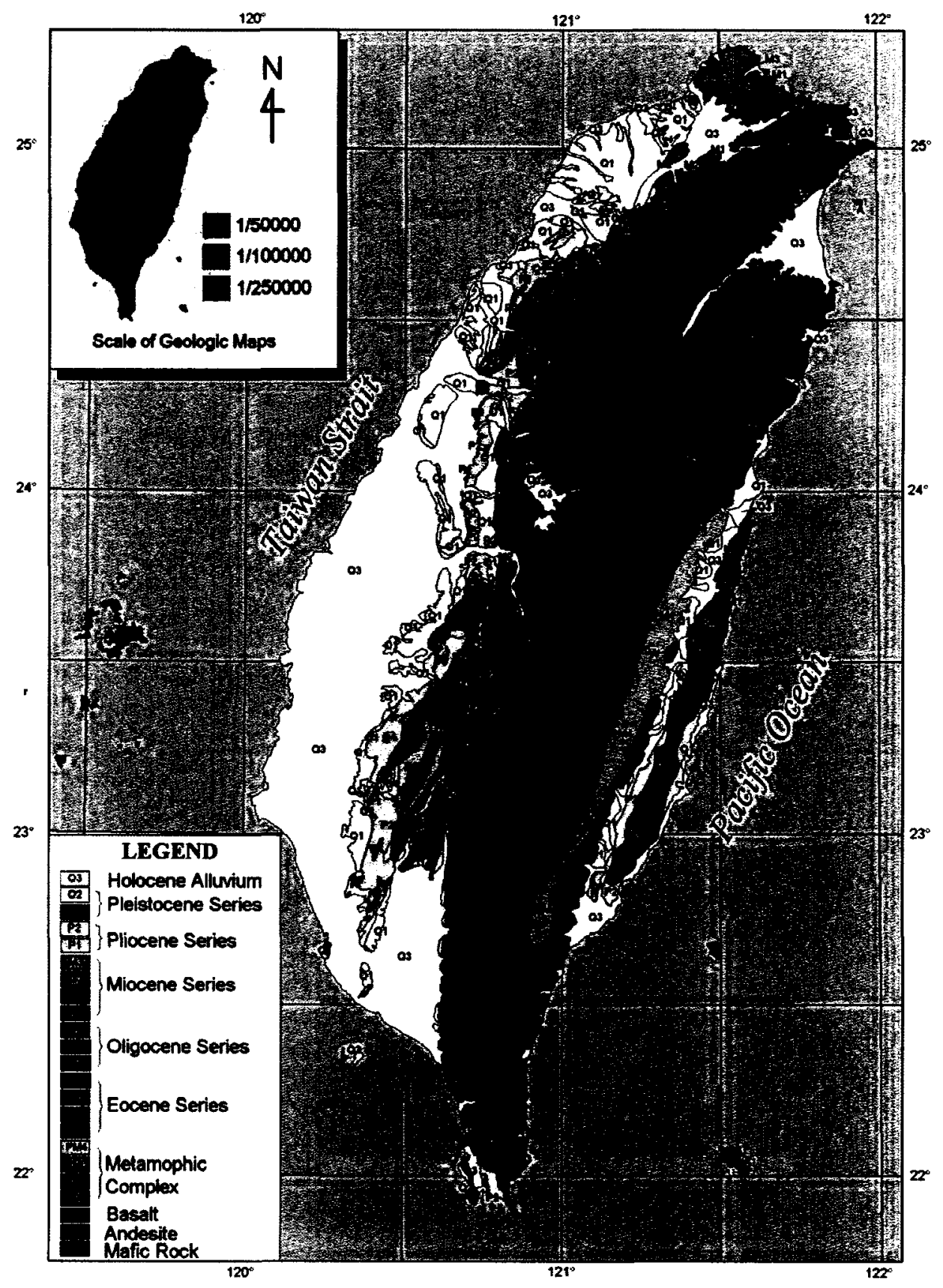

Figure 2.13. Generalized Geologic map of Taiwan (reduced from the 1:250,000 scale geologic map of the Central Geologic Survey). Inset block shows the extent of geologic maps with different scales used in this study. Green area represents the 1:50,000 geologic maps from the Central Geological Survey. Blue area represents the 1:100,000 geologic maps from the Chinese Petroleum Company. Brown area represents 1:250,000 geologic maps from the Central Geologic Survey. After Lee. et al., 2001a. 


\section{Chapter 3}

\section{AVAILABLE DATA AND PROCESSING PROCEDURES}

\subsection{Introduction}

This chapter first describes the seismic network of Taiwan and the dataset used in the study. The seismic database was obtained from Lee et al. (2001b). All of the records were retrieved from three-components accelerographs. Data quality control was first applied to the whole dataset to select the most appropriate records for further processing. Then, the envelope function was calculated for each record. With the help of the envelope function, the shear wave window of the accelerogram was defined and then tapered by a boxcar window function. Finally, the Fourier amplitude acceleration spectrum and pseudo acceleration spectrum were computed.

\subsection{Seismic Network and Data Set}

The Central Weather Bureau (CWB) of Taiwan hosts the Taiwan Strong-Motion Instrumentation Program (TSMIP) which consists of a total of 640 digital accelerographs deployed at "free-field" sites. Among these 640 accelerographs, 439 of them are A800, A900 \& A900A type manufactured by Geotech Instruments; 163 of them are IDS \& IDSA type from Terra Tech; and 38 of them are ETNA \& K2 type made by Kinemetrics (Lee et al., 2001b). The installation of the network was completed in 1996. During the 1999 Chi-Chi earthquake TSMIP recorded the most extensive set of strong-motion data from a single event in the history of seismology. 441 digital records 
of the main shock were re-processed by Lee et al. (2001b) after the pre-publication of the Chi-Chi main shock data (Lee et al., 1999). Comparing with the previous publication, significant improvements were achieved by the re-processing in the following aspects.

1. The coordinates of $85 \%$ of the stations have been verified and confirmed.

2. DC offset was removed by using the computation of the pre-event signal to help define the baseline.

3. Timing correction was applied to stations with a GPS timing device.

\subsection{Data Processing Procedures}

The seismic data used in this study are based on the most recently published data by Lee et al. (2001b). The general data processing procedure is demonstrated in a flow-chart (Figure 3.1), and described as follows.

\section{- Data Quality Control}

411 out of 441 seismograms (about $95 \%$ ) are processed and used in this study. According to Lee et al. (2001b), all of the 441 seismograms are classified into 4 categories: class $\mathrm{A}, \mathrm{B}, \mathrm{C}$ and $\mathrm{D}$ based on their quality. Data belonging to class A category are the most reliable while class $\mathrm{D}$ data have the poorest quality, due to missing portions of the trace, incorrect recording time or signal glitches. The incorrect or incomplete recording time is the most significant problem in dataset, leading to several records that cannot be used. Although there are many inherent deficiencies for class D data, some of them were selected for our database based on the following criteria: no missing trace portion, all three components of the record are complete without timing error, and there 
are no obvious saturation effects. All of the used seismic stations are listed in Table 3.1 and shown in Figure 3.2, where the stations were classified as rock sites (square) or soil sites (up-triangle) according to the shear wave velocity in the top 30 meters.

Table 3.1: Stations used in this study

\begin{tabular}{|c|c|c|c|c|c|c|c|c|c|c|}
\hline Station & Latitude & Longitude & Model & Count/G & EpiDist & Drup & $V_{s 30}$ & PEER & RSS & Geo \\
\hline ALS & 23.5103 & 120.8052 & A800 & 2048 & 38.8 & 10.8 & 553.4 & C & $\mathbf{R}$ & $\mathrm{C}$ \\
\hline CHK & 23.0992 & 121.3653 & A800 & 2048 & 102.2 & 63.53 & 304.7 & $\mathrm{D}$ & $\mathrm{S}$ & $\mathrm{D}$ \\
\hline CHY & 23.4977 & 120.4245 & A800 & 2048 & 55.5 & $30.04^{*}$ & $\mathrm{~N} / \mathrm{A}$ & $\overline{\mathrm{N}} / \mathrm{A}$ & $\bar{N} / \mathrm{A}$ & $\overline{\mathrm{D}}$ \\
\hline CHYO02 & 23.7192 & 120.4125 & IDSA & 16384 & 42.4 & 24.98 & 235.1 & $\mathrm{D}$ & $\mathrm{S}$ & $\mathbf{E}$ \\
\hline CHY004 & 23.6013 & 120.1715 & IDSA & 16384 & 70.2 & 47.34 & 271.3 & $\overline{\mathrm{D}}$ & $\overline{\mathbf{R}}$ & $\bar{E}$ \\
\hline CHY006 & 23.5815 & 120.552 & IDSA & 16384 & 39.9 & 9.77 & 438.2 & $\mathrm{C}$ & $\mathbf{R}$ & $\mathrm{D}$ \\
\hline CHY008 & 23.4853 & 120.2692 & IDSA & 16384 & 68.2 & 40.44 & 210.7 & $\bar{D}$ & $\mathrm{~S}$ & $\bar{E}$ \\
\hline CHY010 & 23.4653 & 120.544 & IDSA & 16384 & 50.9 & 19.96 & 518.7 & $\mathrm{C}$ & $\mathbf{R}$ & $\bar{D}$ \\
\hline CHY012 & 23.3328 & 120.1523 & IDSA & 16384 & 88.2 & 59.05 & 198.4 & $\mathrm{D}$ & $\mathrm{S}$ & $E$ \\
\hline CHY014 & 23.2963 & 120.5828 & IDSA & 16384 & 66.3 & 34.18 & 528.8 & $\mathrm{C}$ & $\mathbf{R}$ & $\bar{D}$ \\
\hline CHY015 & 23.355 & 120.4052 & IDSA & 16384 & 68.9 & 38.14 & 228.7 & $\mathrm{D}$ & $\mathrm{S}$ & $\mathrm{D}$ \\
\hline CHY016 & 23.2212 & 120.1532 & IDSA & 16384 & 96.8 & 66.65 & 200.9 & $\mathrm{D}$ & 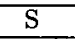 & $\mathrm{E}$ \\
\hline CHY017 & 23.2147 & 120.268 & IDSA & 16384 & 89.8 & 59.09 & 190.6 & $\bar{D}$ & $\bar{S}$ & $\mathrm{E}$ \\
\hline CHY019 & 23.1795 & 120.4778 & IDSA & 16384 & 82.2 & 50.53 & 466 & $\mathrm{C}$ & $\mathbf{R}$ & D \\
\hline CHY022 & 23.0457 & 120.4615 & IDSA & 16384 & 96.6 & 64.15 & 472.1 & $\mathrm{C}$ & $\mathrm{R}$ & $\mathrm{C}$ \\
\hline CHY023 & 22.9655 & 120.28 & IDSA & 16384 & 112.4 & 81.28 & 249.1 & $\mathrm{D}$ & $\mathrm{S}$ & $\mathrm{D}$ \\
\hline CHY024 & 23.757 & 120.6062 & A900 & 16384 & 22.8 & 9.64 & 427.7 & $\mathrm{C}$ & $\mathrm{S}$ & $\mathrm{D}$ \\
\hline CHY025 & 23.7795 & 120.5137 & A900 & 16384 & 30.5 & 19.09 & 277.5 & $\mathrm{D}$ & $\mathrm{S}$ & $\mathrm{E}$ \\
\hline $\mathrm{CHY} 026$ & 23.7987 & 120.4113 & A900 & 16384 & 40.1 & 29.53 & 226 & $\mathrm{D}$ & $\mathrm{S}$ & $E$ \\
\hline CHY027 & 23.752 & 120.2468 & A900 & 16384 & 57.6 & 42 & 210 & $\mathrm{D}$ & $\mathrm{R}$ & $\mathrm{E}$ \\
\hline CHY028 & 23.632 & 120.6052 & A900 & 16384 & 32.1 & 3.14 & 542.6 & $\mathrm{C}$ & $\mathrm{R}$ & $\bar{D}$ \\
\hline CHY029 & 23.6135 & 120.5282 & A900 & 16384 & 38.9 & 10.97 & 544.7 & $\mathrm{C}$ & $\mathrm{R}$ & $\mathrm{C}$ \\
\hline CHY032 & 23.5799 & 120.2944 & A900 & 16384 & 60.1 & 35.44 & 192.7 & $\overline{\mathrm{D}}$ & $\mathrm{S}$ & $\mathrm{E}$ \\
\hline CHY033 & 23.5407 & 120.2153 & A900 & 16384 & 69.3 & 43.83 & 197.6 & $\mathrm{D}$ & $\mathrm{S}$ & $\mathrm{E}$ \\
\hline CHY034 & 23.5212 & 120.5443 & $\overline{\mathrm{IDSA}}$ & 16384 & 45.7 & 14.82 & 378.8 & $\mathrm{C}$ & $\bar{R}$ & $\overline{\mathrm{D}}$ \\
\hline CHY035 & 23.52 & 120.584 & A900A & 16384 & 43.6 & 12.65 & 523.7 & $\mathrm{C}$ & $\bar{R}$ & $\mathrm{D}$ \\
\hline CHY036 & 23.6073 & 120.4788 & A900 & 16384 & 43.1 & 16.06 & 233.1 & $\mathrm{D}$ & $\mathrm{R}$ & $\mathrm{D}$ \\
\hline CHY039 & 23.5207 & 120.344 & A900 & 16384 & 59.8 & 31.88 & 201.2 & $\mathrm{D}$ & $\mathrm{S}$ & $\overline{\mathrm{E}}$ \\
\hline CHY041 & 23.4388 & 120.5957 & A900 & 16384 & 51.1 & 19.83 & 492.3 & $\mathrm{C}$ & $\mathrm{R}$ & $\mathrm{D}$ \\
\hline CHY042 & 23.3583 & 120.5833 & A900 & $1638 \overline{4}$ & 59.8 & 28.17 & 605.6 & $\mathrm{C}$ & $\overline{\mathrm{S}}$ & $?$ \\
\hline CHY044 & 23.3832 & 120.1635 & A900 & 16384 & 83.7 & 55.14 & 194.5 & $\mathrm{D}$ & $\mathrm{S}$ & $\mathrm{E}$ \\
\hline CHY046 & 23.4765 & 120.4632 & A900 & 16384 & 54.6 & 24.11 & 444.6 & $\mathrm{C}$ & $\bar{R}$ & $\mathrm{C}$ \\
\hline CHY047 & 23.4938 & 120.4468 & IDSA & 16384 & 54.2 & 24.14 & 282.5 & $\bar{D}$ & $\mathrm{~S}$ & $\mathrm{D}$ \\
\hline CHY050 & 23.2803 & 120.4083 & A900 & 16384 & 75.6 & 44.76 & 421.7 & $\mathrm{C}$ & $\mathbf{R}$ & $\mathrm{C}$ \\
\hline CHY052 & 23.2878 & 120.501 & IDSA & 16384 & 70.3 & 39.02 & 547.8 & $\mathrm{C}$ & $\mathrm{R}$ & $\mathrm{C}$ \\
\hline CHY054 & 23.3077 & 120.3097 & A900 & 16384 & 79 & 48.49 & 172.1 & $\mathbf{E}$ & $\mathbf{R}$ & $\mathrm{E}$ \\
\hline CHY055 & 23.2698 & 120.2705 & $\overline{\mathrm{IDSA}}$ & 16384 & 84.8 & 54.31 & 225.8 & $\mathrm{D}$ & $S$ & $\mathrm{E}$ \\
\hline CHY057 & 23.1495 & 120.4098 & A900 & 16384 & 88.2 & 56.93 & 411.5 & $\mathrm{C}$ & $\overline{\mathrm{R}}$ & $\mathrm{C}$ \\
\hline CHY058 & 23.1725 & 120.3192 & A900 & 16384 & 90.6 & 59.8 & 233 & $\mathrm{D}$ & $\mathrm{S}$ & $\mathrm{D}$ \\
\hline CHY059 & 23.184 & 120.1025 & A900 & 16384 & 103.3 & 73.27 & 191.1 & $\mathrm{D}$ & 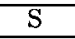 & $\mathrm{E}$ \\
\hline CHY060 & 23.1243 & 120.2392 & A900A & 16384 & 99.6 & 68.86 & 228.9 & $\mathrm{D}$ & $\mathrm{S}$ & $\mathrm{E}$ \\
\hline CHY061 & 23.0768 & 120.5107 & A900 & 16384 & 91.7 & 58.75 & 478.5 & $\mathrm{C}$ & $\mathbf{R}$ & $\mathrm{C}$ \\
\hline CHY063 & 23.027 & 120.3402 & A900 & 16384 & 103.5 & 72.23 & 246.9 & $\bar{D}$ & $\mathbf{R}$ & $\mathrm{D}$ \\
\hline CHY065 & 22.906 & 120.345 & A900 & 16384 & 115.5 & 83.43 & 332 & D & $\mathbf{R}$ & $\mathrm{D}$ \\
\hline
\end{tabular}

Continued... 


\begin{tabular}{|c|c|c|c|c|c|c|c|c|c|c|}
\hline Station & Latitude & Longitude & Model & Count/G & EpiDist & Drup & $V_{s 30}$ & PEER & RSS & Geo \\
\hline CHY066 & 22.9205 & 120.2078 & IDSA & 16384 & 120.4 & 89.48 & 212 & $\bar{D}$ & $\overline{\mathrm{S}}$ & $\mathrm{E}$ \\
\hline CHY067 & 22.999 & 120.1837 & IDSA & 16384 & 114.3 & 83.56 & 228 & $\mathrm{D}$ & $\mathrm{S}$ & $\mathrm{E}$ \\
\hline CHY069 & 22.9737 & 120.1815 & A900 & 16384 & 116.8 & 86.02 & 198 & $\mathrm{D}$ & $\mathrm{S}$ & $\mathrm{E}$ \\
\hline CHY070 & 22.9651 & 120.2286 & A900 & 16384 & 115 & 83.61 & 274.1 & $\bar{D}$ & $\mathrm{~S}$ & $\mathrm{D}$ \\
\hline CHY071 & 23.0648 & 120.1645 & A900 & 16384 & 109.4 & 78.74 & 198 & $\bar{D}$ & $\mathrm{~S}$ & $E$ \\
\hline CHY074 & 23.5103 & 120.8052 & A900A & 16384 & 38.8 & 10.8 & 553.4 & $\mathrm{C}$ & $\bar{R}$ & $\mathrm{C}$ \\
\hline CHY075 & 23.5672 & 119.5552 & A900A & 16384 & 131 & $115.8^{*}$ & 409.9 & $\mathrm{C}$ & $\overline{\mathrm{N}} / \mathrm{A}$ & B \\
\hline CHY076 & 23.638 & 120.2217 & A900A & 16384 & 63.8 & 42.16 & 169.8 & $E$ & $\mathrm{~S}$ & $\mathbf{E}$ \\
\hline CHY078 & 23.0402 & 120.2283 & A900A & 16384 & 108 & 77.2 & 160.7 & $\mathrm{E}$ & $\mathrm{S}$ & $\mathrm{D}$ \\
\hline CHY079 & 23.1848 & 120.528 & A900A & 16384 & 79.8 & 47.53 & 519.7 & $\mathrm{C}$ & $\mathrm{R}$ & $\mathrm{C}$ \\
\hline CHY080 & 23.5972 & 120.6777 & A900A & 16384 & 31.7 & 2.69 & 611.1 & $\mathrm{C}$ & $\mathrm{R}$ & $?$ \\
\hline CHY081 & 23.2703 & 120.4965 & A900A & 16384 & 72.3 & 41.67 & 548.6 & $\mathrm{C}$ & $\mathrm{R}$ & $\mathrm{C}$ \\
\hline CHY082 & 23.7237 & 120.2975 & A900 & 16384 & 53.4 & 36.11 & 193.7 & $\bar{D}$ & $\overline{\mathrm{R}}$ & $\mathrm{E}$ \\
\hline $\begin{array}{l}\text { CHY086 } \\
\end{array}$ & 23.351 & 120.5932 & A900A & 16384 & 60.2 & 28.42 & 598.7 & $\mathrm{C}$ & $\mathbf{R}$ & $?$ \\
\hline CHY087 & 23.3845 & 120.519 & A900 & 16384 & 60 & 28.91 & 523.9 & $\mathrm{C}$ & $\mathrm{R}$ & $\mathrm{C}$ \\
\hline CHY088 & 23.3462 & 120.4293 & A900 & 16384 & $68 . \overline{3}$ & 37.48 & $\overline{353.7}$ & $\mathrm{D}$ & $\mathrm{R}$ & $\mathrm{D}$ \\
\hline CHY090 & 23.2673 & 120.2163 & A900 & 16384 & 88.6 & 58.43 & 198 & $\mathrm{D}$ & $\mathrm{S}$ & $\mathrm{E}$ \\
\hline CHY092 & 23.7913 & 120.4783 & IDSA & 16384 & 33.6 & 22.7 & 253.7 & $\bar{D}$ & $\mathrm{~S}$ & $E$ \\
\hline CHY093 & 23.6538 & 120.1472 & IDSA & 16384 & 70.3 & 49.83 & 190.5 & $\mathrm{D}$ & $\overline{\mathrm{R}}$ & $\mathrm{E}$ \\
\hline CHY094 & 23.7935 & 120.3205 & IDSA & 16384 & 49.4 & $\overline{37.12}$ & 221.9 & $\bar{D}$ & $\overline{\mathrm{R}}$ & $\bar{E}$ \\
\hline CHY096 & 22.983 & 120.2327 & IDSA & 16384 & 113.1 & 82.26 & 282.5 & $\mathrm{D}$ & $\mathrm{S}$ & $\mathrm{D}$ \\
\hline CHY099 & 23.1373 & 120.2802 & IDSA & 16384 & 96 & 65.28 & 228.8 & $\mathrm{D}$ & $\mathrm{S}$ & $\mathrm{D}$ \\
\hline $\begin{array}{l}\text { CHY100 } \\
\end{array}$ & 23.2272 & 120.3418 & IDSA & 16384 & 84.3 & 53.46 & 230.1 & $\bar{D}$ & $\mathrm{~S}$ & $\mathrm{D}$ \\
\hline CHY101 & 23.6862 & 120.5622 & A900A & 16384 & 30.9 & 9.96 & 258.9 & $\mathrm{D}$ & $\mathrm{S}$ & $\mathrm{D}$ \\
\hline CHY102 & 23.2455 & 120.6138 & A900A & 16384 & 70.7 & 37.72 & 592.4 & $\mathrm{C}$ & $\overline{\mathrm{R}}$ & $\mathrm{B}$ \\
\hline CHY104 & 23.6695 & 120.4648 & IDSA & 16384 & 40.1 & 18.04 & 223.2 & $\bar{D}$ & $\mathrm{~S}$ & $\mathrm{E}$ \\
\hline CHY107 & 23.2988 & 120.2897 & IDSA & 16384 & 81.1 & 50.62 & 175.7 & $\bar{E}$ & $\overline{\mathrm{R}}$ & $\mathrm{E}$ \\
\hline CHY109 & 23.2517 & 120.5295 & A800 & 2048 & 72.8 & 41.03 & 525.8 & $\mathrm{C}$ & $\bar{R}$ & $\mathrm{C}$ \\
\hline CHY110 & 23.2517 & 120.5295 & $\overline{\mathrm{A} 800}$ & 2048 & 72.8 & 41.03 & 521.5 & $\mathrm{C}$ & $\overline{\mathrm{R}}$ & $\mathrm{C}$ \\
\hline CHY111 & 23.7912 & 120.2273 & IDSA & 16384 & 58.8 & $48.02^{*}$ & 276.3 & $\bar{D}$ & N/A & $\mathrm{E}$ \\
\hline CHY112 & 23.7035 & 120.183 & IDSA & 16384 & 65.2 & $52.03^{*}$ & 239.7 & $\mathrm{D}$ & N/A & $\mathrm{E}$ \\
\hline CHY114 & 23.0372 & 120.1187 & IDSA & 16384 & 114.7 & $86.65^{*}$ & 225 & $\bar{D}$ & $\mathrm{~N} / \mathrm{A}$ & $\mathrm{E}$ \\
\hline CHY115 & 23.1543 & 120.0967 & IDSA & 16384 & 106.1 & $79.23^{*}$ & 225 & $\mathrm{D}$ & N/A & $\mathrm{E}$ \\
\hline CHY116 & 23.0775 & 120.1082 & $\overline{I D S A}$ & 16384 & 111.8 & 125.03 & 198 & $\bar{D}$ & $\mathrm{~S}$ & $\mathrm{E}$ \\
\hline ENA & 24.428 & 121.7407 & A800 & 2048 & 114.5 & 66.88 & 510.2 & $\mathrm{C}$ & $\overline{\mathrm{R}}$ & $\mathrm{B}$ \\
\hline ESL & 23.8137 & 121.4328 & $\mathrm{~A} 800$ & 2048 & 64.7 & 44.54 & 497.3 & $\mathrm{C}$ & $\mathrm{R}$ & $\mathrm{D}$ \\
\hline HSN & 24.8022 & 120.9695 & A800 & 2048 & 105.7 & 55.49 & 304.7 & $\bar{D}$ & $\mathrm{~S}$ & $\bar{D}$ \\
\hline HWA & 23.977 & 121.605 & A800 & 2048 & 83 & 55.59 & 238.7 & $\mathrm{D}$ & $\mathrm{R}$ & $\overline{\mathrm{D}}$ \\
\hline HWA002 & 23.6012 & 121.5122 & IDS & 16384 & 78.1 & 56.93 & 391.2 & $\mathrm{C}$ & $\mathbf{R}$ & $\mathrm{C}$ \\
\hline HWA003 & 23.4768 & 121.497 & IDS & 16384 & 82.9 & 56.14 & 393.8 & $\mathrm{C}$ & $\overline{\mathrm{S}}$ & $\mathrm{D}$ \\
\hline HWA005 & 23.6608 & 121.414 & IDS & 16384 & 66.4 & 47.58 & 482.9 & $\mathrm{C}$ & $\bar{R}$ & $\mathrm{D}$ \\
\hline HWA006 & 23.6732 & 121.4173 & IDS & 16384 & 66.3 & 47.86 & 484.6 & $\mathrm{C}$ & $\mathbf{R}$ & $\mathrm{D}$ \\
\hline HWA007 & 23.9877 & 121.6173 & A900 & 16384 & 84.4 & 56.3 & 249.1 & $\overline{\mathrm{D}}$ & $\mathrm{S}$ & $\mathrm{D}$ \\
\hline HWA009 & 23.9925 & 121.6165 & A900 & 16384 & 84.5 & 56.06 & 313.8 & $\mathrm{D}$ & $\bar{R}$ & $\bar{D}$ \\
\hline HWA011 & $23 . \overline{9962}$ & 121.5858 & A900 & 16384 & 81.5 & 53.19 & 236.6 & D & $\mathbf{S}$ & $\mathrm{D}$ \\
\hline HWA012 & $23.99 \overline{3}$ & 121.6233 & A900 & 16384 & 85.1 & 56.65 & 270.1 & $\mathrm{D}$ & $\bar{S}$ & $\mathrm{D}$ \\
\hline HWA013 & 23.978 & 121.591 & A900 & 16384 & 81.6 & 54.32 & 227.2 & $\bar{D}$ & $\mathrm{~S}$ & $\mathrm{D}$ \\
\hline HWA014 & 23.9732 & 121.5993 & A900 & 16384 & 82.4 & $5 \overline{5.24}$ & 225.1 & $\mathrm{D}$ & $\mathrm{S}$ & $\mathrm{D}$ \\
\hline HWA015 & 23.9757 & 121.553 & $\mathrm{~A} 900 \mathrm{~A}$ & 16384 & 77.8 & 51.12 & 322.9 & $\bar{D}$ & $\mathrm{~S}$ & $\mathrm{D}$ \\
\hline HWA016 & 23.9648 & 121.56 & $\overline{\mathrm{A} 900}$ & 16384 & 78.3 & 52.18 & 332 & $\bar{D}$ & $\mathrm{~S}$ & $\mathrm{D}$ \\
\hline HWA017 & 23.9497 & 121.5392 & A900 & 16384 & 76 & 51.11 & 435.8 & $\mathrm{C}$ & $\bar{S}$ & $\mathrm{D}$ \\
\hline HWA019 & 23.977 & 121.605 & A900A & 16384 & 83 & $\overline{55.59}$ & 238.7 & D & $\mathrm{S}$ & $\mathrm{D}$ \\
\hline HWA020 & 23.8137 & 121.4328 & A900A & 16384 & 64.7 & 44.54 & 497.3 & $\mathrm{C}$ & $\bar{R}$ & $\mathrm{D}$ \\
\hline HWA022 & 24.2675 & 121.7325 & A900A & 16384 & 105.1 & 62.07 & 386.1 & $\mathrm{C}$ & $\mathbf{R}$ & $\mathrm{C}$ \\
\hline $\begin{array}{l}\text { HWA023 } \\
\end{array}$ & 24.08 & 121.5955 & $\mathrm{~A} 900 \mathrm{~A}$ & 16384 & 84.6 & 51.15 & 438.3 & $\mathrm{C}$ & $\mathrm{R}$ & $\mathrm{B}$ \\
\hline
\end{tabular}

Continued... 


\begin{tabular}{|c|c|c|c|c|c|c|c|c|c|c|}
\hline Station & Latitude & Longitude & Model & Count/G & EpiDist & Drup & $V_{s 30}$ & PEER & RSS & Geo \\
\hline HWA024 & 23.3522 & $\overline{121.297}$ & $\overline{\mathrm{A} 900 \mathrm{~A}}$ & 16384 & $\overline{75.8}$ & 43.15 & 559.5 & $\overline{\mathrm{C}}$ & $\overline{\mathbf{R}}$ & $\bar{B}$ \\
\hline HWA025 & 24.163 & 121.6447 & A900A & 16384 & 92.3 & 53.79 & 409.8 & $\mathrm{C}$ & $\mathrm{R}$ & $\mathrm{D}$ \\
\hline HWA026 & 24.1185 & 121.6165 & $\overline{\mathrm{A} 900 \bar{A}}$ & 16384 & 87.9 & 51.96 & 457.5 & $\mathrm{C}$ & $\mathbf{R}$ & $\mathrm{B}$ \\
\hline HWA027 & 24.055 & 121.591 & A900A & 16384 & 83.4 & 51.62 & 274.1 & $\bar{D}$ & $\bar{R}$ & $\bar{D}$ \\
\hline HWA028 & 24.0172 & 121.6013 & A900A & 16384 & 83.5 & 53.84 & 236.6 & $\bar{D}$ & $\mathrm{~s}$ & $\mathrm{D}$ \\
\hline HWA029 & 23.9373 & 121.5702 & A900A & 16384 & 78.9 & 54.29 & 291.2 & $\mathrm{D}$ & $\mathrm{S}$ & $\bar{D}$ \\
\hline HWA030 & 23.7852 & 121.4488 & A900A & 16384 & 66.7 & 46.95 & 480.9 & $\mathrm{C}$ & $\mathbf{R}$ & $\bar{D}$ \\
\hline HWA031 & 23.7653 & 121.4922 & A900A & 16384 & 71.4 & 51.46 & 461.9 & $\mathrm{C}$ & $\mathrm{S}$ & $\mathrm{D}$ \\
\hline HWA032 & $\begin{array}{l}23.7108 \\
\end{array}$ & 121.412 & A900A & 16384 & 64.6 & 47.16 & 492.2 & $\mathrm{C}$ & $\mathbf{R}$ & $\mathrm{D}$ \\
\hline HWA033 & 23.6867 & 121.4747 & A900A & $16 \overline{884}$ & 71.5 & 53.21 & 395.6 & $\mathrm{C}$ & $\mathrm{R}$ & $\mathrm{C}$ \\
\hline HWA034 & 23.5905 & $\overline{121.3772}$ & A900A & 16384 & 66.1 & 44.32 & 379.2 & $\mathrm{C}$ & $\overline{\mathrm{R}}$ & $\bar{D}$ \\
\hline HWA035 & 23.732 & 121.4362 & A900A & 16384 & 66.4 & 48.35 & 482.4 & $\mathrm{C}$ & $\bar{R}$ & D \\
\hline HWA036 & 23.4995 & 121.367 & A900A & 16384 & 70.3 & 43.8 & 474.1 & $\mathrm{C}$ & $\mathrm{S}$ & $\mathrm{D}$ \\
\hline HWA037 & 23.4542 & 121.384 & A900A & 16384 & 74.7 & 46.2 & 469.3 & $\bar{C}$ & $\overline{\mathrm{R}}$ & $\mathrm{D}$ \\
\hline HWA038 & 23.4615 & 121.3445 & A900A & 16384 & 71 & 42.54 & 525.2 & $\mathrm{C}$ & $\bar{R}$ & $\mathrm{C}$ \\
\hline HWA039 & 23.3845 & 121.3523 & $\overline{\mathrm{A} 900 \mathrm{~A}}$ & 16384 & 77.2 & 45.89 & 491.7 & $\mathrm{C}$ & $\bar{R}$ & $\mathrm{D}$ \\
\hline HWA041 & 23.2675 & 121.2942 & A900A & 16384 & 82.8 & 47.76 & 496.4 & $\mathrm{C}$ & $\mathrm{S}$ & $\mathrm{D}$ \\
\hline HWA043 & 23.7092 & 121.54 & A900A & 16384 & 77.3 & 58.05 & 225.1 & $\bar{D}$ & $\overline{\mathrm{R}}$ & $\overline{\mathrm{D}}$ \\
\hline HWA044 & 23.6538 & 121.5268 & A900A & 16384 & 77.6 & 58.22 & 393.8 & $\mathrm{C}$ & $\bar{R}$ & $\bar{D}$ \\
\hline HWA045 & 24.3095 & 121.7405 & IDS & 16384 & 107.8 & 63.43 & 252.2 & $\mathrm{D}$ & $\mathrm{R}$ & $\mathrm{D}$ \\
\hline HWA046 & 24.1492 & 121.6213 & IDS & 16384 & 89.5 & 51.8 & 460.9 & $\mathrm{C}$ & $\overline{\mathrm{R}}$ & $\bar{B}$ \\
\hline HWA048 & 24.0113 & 121.5715 & IDS & 16384 & 80.4 & 51.41 & 270.1 & $\bar{D}$ & $\mathrm{R}$ & D \\
\hline HWA 049 & 23.9952 & 121.5577 & IDS & 16384 & 78.6 & 50.76 & 377.5 & $\mathrm{C}$ & $\mathrm{R}$ & $\mathrm{D}$ \\
\hline HWA050 & 23.9897 & 121.584 & IDS & 16384 & 81.1 & 53.27 & 234.7 & $\bar{D}$ & $\mathrm{R}$ & $\mathrm{D}$ \\
\hline HWA051 & 23.8703 & 121.548 & IDS & 16384 & 76.2 & 53.56 & 345.2 & $\mathrm{D}$ & $\mathrm{R}$ & D \\
\hline HWA055 & 23.3232 & 121.3323 & IDS & 16384 & 80.6 & 47.46 & 492.1 & $\mathrm{C}$ & $\overline{\mathrm{R}}$ & $\bar{D}$ \\
\hline HWA056 & 24.1795 & 121.5077 & A900A & 16384 & 80.3 & 41.1 & 511.3 & $\mathrm{C}$ & $\mathrm{R}$ & $\mathrm{B}$ \\
\hline HWA057 & 24.16 & 121.61 & A900A & 16384 & 88.9 & 50.6 & 581.3 & $\bar{C}$ & $\overline{\mathrm{R}}$ & $\mathrm{N} / \mathrm{A}$ \\
\hline HWA058 & 23.9672 & 121.484 & $\overline{\mathrm{A} 900 \mathrm{~A}}$ & 16384 & 70.7 & 45.77 & 542.1 & $\mathrm{C}$ & $\mathrm{R}$ & N/A \\
\hline HWA059 & 23.8713 & 121.5005 & A900A & 16384 & 71.4 & 49.15 & 400.4 & $\mathrm{C}$ & $\mathrm{R}$ & N/A \\
\hline HWA060 & 23.8703 & 121.59 & A900A & 16384 & 80.5 & 57.51 & 402.4 & $\mathrm{C}$ & $\bar{R}$ & N/A \\
\hline HWA2 & 23.977 & 121.605 & A900A & 16384 & 83 & 55.59 & 238.7 & $\bar{D}$ & $\overline{\mathrm{R}}$ & $\mathrm{N} / \mathrm{A}$ \\
\hline ILA001 & 24.8827 & 121.836 & IDS & 16384 & 154.5 & 103.2 & 382.8 & $\mathrm{C}$ & $\mathrm{R}$ & D \\
\hline ILA002 & 24.845 & 121.7973 & IDS & 16384 & 148.8 & 97.58 & 225.6 & $\bar{D}$ & $\mathrm{~S}$ & $\bar{D}$ \\
\hline ILA003 & 24.7982 & 121.7813 & IDS & 16384 & 143.9 & 92.81 & 263.8 & $\mathrm{D}$ & $\mathrm{S}$ & $E$ \\
\hline ILA004 & 24.745 & 121.7828 & IDS & 16384 & 139.9 & 88.89 & 124.3 & $\mathbf{E}$ & $\mathrm{S}$ & $\mathrm{E}$ \\
\hline ILA005 & 24.6978 & 121.8052 & IDS & 16384 & 138 & 87.2 & 239.3 & $\mathrm{D}$ & $\mathrm{S}$ & $\bar{D}$ \\
\hline ILA006 & 24.6412 & 121.825 & IDS & 16384 & 135.4 & 85.07 & 279.4 & $\mathrm{D}$ & $\mathrm{S}$ & $\mathrm{D}$ \\
\hline ILA007 & 24.5943 & 121.8462 & IDS & 16384 & 133.8 & 84.07 & 384 & $\mathrm{C}$ & $\mathrm{S}$ & $\mathrm{D}$ \\
\hline ILA008 & 24.7088 & 121.7628 & IDS & 16384 & 135.6 & 84.8 & 226 & $\mathrm{D}$ & $\overline{\mathrm{R}}$ & $\mathbf{E}$ \\
\hline ILA010 & 24.619 & 121.7812 & IDS & 16384 & 130.4 & 80.18 & 383.1 & $\mathrm{C}$ & $\overline{\mathrm{R}}$ & $\mathrm{C}$ \\
\hline ILA012 & 24.7807 & 121.7335 & IDS & 16384 & 139.2 & 88.18 & 233 & $\mathrm{D}$ & $\mathrm{S}$ & $\mathrm{D}$ \\
\hline ILA013 & 24.735 & 121.7294 & IDS & 16384 & 135.3 & 84.12 & 215 & $\bar{D}$ & $\mathrm{~S}$ & $\mathrm{D}$ \\
\hline ILA014 & 24.6945 & 121.7202 & IDS & 16384 & 131.4 & 80.67 & 300.8 & $\mathrm{D}$ & $\mathrm{R}$ & $\bar{D}$ \\
\hline ILA015 & 24.7807 & 121.6912 & IDS & 16384 & 136.3 & 85.4 & 493.2 & $\mathrm{C}$ & $\mathrm{R}$ & $\mathrm{B}$ \\
\hline ILA016 & 24.749 & 121.684 & IDS & 16384 & 133.2 & 82.36 & 271.1 & $\mathrm{D}$ & $\mathrm{S}$ & $\bar{D}$ \\
\hline ILA021 & 24.7135 & 121.644 & IDS & 16384 & 127.6 & 76.9 & 453.2 & $\mathrm{C}$ & $\mathrm{S}$ & $\mathrm{D}$ \\
\hline ILA024 & 24.6448 & 121.588 & IDS & 16384 & 118.2 & 67.81 & 536.6 & $\mathrm{C}$ & $\mathrm{S}$ & $\mathrm{B}$ \\
\hline ILA027 & 24.6893 & 121.7603 & A800 & 2048 & 134 & 83.23 & 215 & $\overline{\mathrm{D}}$ & $\mathrm{S}$ & $\bar{D}$ \\
\hline ILA030 & 24.7278 & 121.755 & A900 & 16384 & 136.5 & 85.62 & 201 & $\mathrm{D}$ & $\mathrm{S}$ & $\mathbf{E}$ \\
\hline ILA031 & 24.5995 & 121.8337 & A900 & 16384 & 133.2 & 83.31 & 649.2 & $\mathrm{C}$ & $\overline{\mathbf{R}}$ & $\bar{B}$ \\
\hline \begin{tabular}{|l|} 
ILA032 \\
\end{tabular} & 24.6242 & 121.8272 & A800 & 2048 & 134.4 & 84.22 & 295.5 & $\mathrm{D}$ & $\mathrm{S}$ & $\mathrm{D}$ \\
\hline \begin{tabular}{|l} 
ILA035 \\
\end{tabular} & 24.8241 & 121.7603 & A800 & 2048 & 144.6 & 93.43 & 236.6 & D & $\mathrm{S}$ & $\mathrm{D}$ \\
\hline ILA036 & 24.7883 & 121.7513 & A900 & 16384 & 141.1 & 89.98 & 225.6 & $\mathrm{D}$ & $\mathrm{S}$ & $\mathrm{D}$ \\
\hline \begin{tabular}{|l|} 
ILA037 \\
\end{tabular} & 24.7455 & 121.7142 & A900 & 16384 & 135 & 84.11 & 210.2 & $\mathrm{D}$ & $\mathrm{S}$ & $\mathrm{D}$ \\
\hline
\end{tabular}

Continued... 


\begin{tabular}{|c|c|c|c|c|c|c|c|c|c|c|}
\hline Station & Latitude & Longitude & Model & Count/G & EpiDist & Drup & $V_{s 30}$ & PEER & RSS & Geo \\
\hline$\overline{\text { ILA039 }}$ & 24.765 & 121.7212 & A800 & 2048 & $\overline{137.1}$ & 86.11 & $\overline{227.2}$ & $\bar{D}$ & $\overline{\mathrm{S}}$ & $\overline{\mathrm{D}}$ \\
\hline ILA041 & 24.7233 & 121.7917 & IDS & 16384 & 138.9 & 87.97 & 196.9 & $\bar{D}$ & $\mathrm{~S}$ & $\mathrm{E}$ \\
\hline ILA042 & 24.6903 & 121.792 & A900 & 16384 & 136.4 & 85.68 & 209.4 & $\bar{D}$ & $\mathrm{~S}$ & $\mathrm{E}$ \\
\hline ILA043 & 24.629 & 121.7347 & $\overline{\mathrm{A}} 8 \overline{00}$ & 2048 & 127.6 & 77.17 & 408.7 & $\mathrm{C}$ & $\overline{\mathrm{R}}$ & $\mathrm{C}$ \\
\hline ILA044 & 24.656 & 121.755 & IDS & 16384 & 131.1 & 80.53 & 158.1 & $\mathrm{E}$ & $\mathrm{S}$ & $\mathrm{D}$ \\
\hline ILA046 & 24.666 & 121.7338 & A900 & 16384 & 130.2 & 79.62 & 396.9 & $\mathrm{C}$ & $\bar{S}$ & $\overline{\mathrm{D}}$ \\
\hline ILA048 & 24.7663 & $\overline{121.7612}$ & IDS & 16384 & 140 & 88.95 & 199.6 & $\overline{\mathrm{D}}$ & $\bar{S}$ & $\mathrm{E}$ \\
\hline ILA049 & 24.7655 & 121.748 & A900A & 16384 & 139 & 87.98 & 215 & $\bar{D}$ & $\mathrm{~S}$ & $\bar{D}$ \\
\hline ILA050 & 24.428 & 121.7407 & A900A & 16384 & 114.5 & 66.88 & 479.8 & $\mathrm{C}$ & $\overline{\mathrm{R}}$ & $\bar{B}$ \\
\hline ILA051 & 24.7212 & 121.667 & A900A & 16384 & 129.8 & 79.03 & 484.3 & $\mathrm{C}$ & $\mathrm{S}$ & $\mathrm{B}$ \\
\hline ILA052 & 24.6092 & 121.8488 & $\overline{A 900 A}$ & 16384 & 135 & 85.13 & 424.2 & $\mathrm{C}$ & $\mathrm{R}$ & $\mathrm{B}$ \\
\hline ILA054 & 24.9732 & 121.9183 & IDSA & 16384 & 167.5 & 116.01 & 408.7 & $\mathrm{C}$ & $\bar{S}$ & $\bar{B}$ \\
\hline ILA055 & 24.7387 & 121.8083 & IDSA & 16384 & 141.3 & 90.3 & 266.8 & $\mathrm{D}$ & $\mathrm{S}$ & $\mathrm{E}$ \\
\hline ILA056 & 24.7622 & 121.8088 & IDSA & 16384 & 143.1 & 92.04 & 223.7 & $\bar{D}$ & $\bar{S}$ & $\mathrm{E}$ \\
\hline ILA059 & 24.6667 & 121.8205 & IDSA & 16384 & 136.8 & 86.33 & 236.8 & $\mathrm{D}$ & $\overline{\mathrm{R}}$ & $\mathrm{E}$ \\
\hline ILA061 & 24.5233 & 121.825 & IDSA & 16384 & 127.5 & 78.55 & 236.6 & $\mathrm{D}$ & $\mathrm{S}$ & $\bar{D}$ \\
\hline ILA062 & 24.4677 & 121.7933 & IDSA & 16384 & 121.3 & 73.22 & 270.1 & $\mathrm{D}$ & $\mathrm{R}$ & $\mathrm{D}$ \\
\hline ILA063 & 24.6157 & 121.518 & IDSA & 16384 & 111 & 61.06 & 565.1 & $\mathrm{C}$ & $\mathbf{R}$ & $\mathrm{B}$ \\
\hline ILA064 & 24.477 & 121.7787 & IDSA & 16384 & 120.7 & 72.33 & 398 & $\mathrm{C}$ & $\mathrm{S}$ & D \\
\hline ILA066 & 24.4473 & 121.7707 & IDSA & 16384 & 118.2 & 70.35 & 295.6 & $\mathrm{D}$ & $\mathbf{R}$ & $\mathrm{D}$ \\
\hline ILA067 & 24.44 & 121.3728 & A900A & 16384 & 86.7 & 38.82 & 610.6 & $\mathrm{C}$ & $\bar{R}$ & N/A \\
\hline KAU001 & 23.1618 & 120.6355 & IDSA & 16384 & 79.2 & 44.93 & 542.3 & $\mathrm{C}$ & $\overline{\mathbf{R}}$ & $\mathrm{D}$ \\
\hline KAU003 & 22.628 & $1 \overline{20.2573}$ & IDSA & 16384 & 147.3 & 114.44 & $91 \overline{3} .8$ & $\mathrm{~B}$ & $\mathrm{~S}$ & $\bar{D}$ \\
\hline KAU006 & 22.591 & 120.3173 & A900A & 16384 & 149 & 114.92 & 218.5 & $\overline{\mathrm{D}}$ & $\mathrm{S}$ & $\mathrm{E}$ \\
\hline KAU007 & 22.6462 & 120.3585 & A900 & 16384 & 141.8 & 107.57 & 246.2 & $\bar{D}$ & $\mathrm{~S}$ & $\bar{D}$ \\
\hline KAU008 & 22.6295 & 120.3672 & A900A & 16384 & 143.3 & 108.75 & 285.9 & $\bar{D}$ & $\mathrm{~S}$ & $\mathrm{D}$ \\
\hline KAU010 & 22.7873 & 120.279 & A900 & 16384 & 130.2 & 98.16 & 198 & $\mathrm{D}$ & $\mathrm{S}$ & $\mathrm{E}$ \\
\hline KAU011 & 22.7613 & 120.2558 & A900 & 16384 & 133.8 & 101.85 & 155.3 & $\mathrm{E}$ & $\mathrm{S}$ & $\mathrm{E}$ \\
\hline KAU012 & 22.8797 & 120.3707 & A900 & 16384 & 117.1 & 84.61 & 402.4 & $\overline{\mathrm{C}}$ & $\mathrm{S}$ & $\bar{D}$ \\
\hline KAU015 & 22.656 & 120.3317 & A900 & 16384 & 141.7 & $10 \overline{07.97}$ & 309.2 & $\mathrm{D}$ & $\mathrm{S}$ & $\mathrm{D}$ \\
\hline KAU017 & 22.509 & 120.3862 & A900 & 16384 & 155.5 & $126.55^{*}$ & 284.7 & $\bar{D}$ & N/A & $\mathrm{E}$ \\
\hline KAU018 & 22.891 & 120.4738 & A900 & 16384 & 112.4 & 78.41 & 420.6 & $\mathrm{C}$ & $S$ & $\bar{D}$ \\
\hline KAU020 & 22.902 & 120.5352 & A900 & 16384 & 109.5 & 74.48 & 373.3 & $\mathrm{C}$ & $\mathrm{S}$ & $\mathrm{D}$ \\
\hline KAU022 & 22.6707 & 120.491 & A900 & 16384 & 135.5 & 98.88 & 199 & $\bar{D}$ & $\mathrm{~S}$ & $\mathrm{E}$ \\
\hline KAU030 & 22.6108 & 120.5588 & A900 & 16384 & 140.6 & 102.09 & 198.1 & $\mathrm{D}$ & $\mathrm{S}$ & $\mathrm{E}$ \\
\hline KAU032 & 22.5462 & 120.4528 & A900 & 16384 & 149.8 & 112.81 & 198 & $\overline{\mathrm{D}}$ & $\bar{S}$ & $\overline{\mathrm{E}}$ \\
\hline KAU037 & 22.2617 & 120.6432 & A900 & 16384 & 177.8 & 136.47 & 283.2 & $\bar{D}$ & $\mathrm{~S}$ & $\mathrm{D}$ \\
\hline KAU044 & 22.4397 & 120.5028 & A900 & 16384 & 160.2 & 121.61 & 198 & $\bar{D}$ & $S$ & $\mathrm{E}$ \\
\hline KAU045 & 22.5678 & 120.308 & A900A & 16384 & 151.7 & 119.22 & 150.1 & $\mathrm{E}$ & N/A & $\bar{E}$ \\
\hline KAU047 & 23.0817 & 120.5827 & A900A & 16384 & 89 & 54.95 & $5 \overline{33}$ & $\mathrm{C}$ & $\mathrm{R}$ & B \\
\hline KAU048 & 22.7252 & 120.4908 & $\overline{A 900 A}$ & 16384 & 129.6 & 93.61 & 286.8 & $\bar{D}$ & $\bar{S}$ & $\bar{D}$ \\
\hline KAU050 & 23.1625 & 120.7573 & A900A & 16384 & 77.4 & 40.49 & 596.6 & $\mathrm{C}$ & $\overline{\mathrm{R}}$ & $\mathrm{B}$ \\
\hline KAU054 & 23.2777 & $120 . \overline{7128}$ & A900 & 16384 & 65.1 & 30.85 & 553.8 & $\mathrm{C}$ & $\overline{\mathrm{R}}$ & $\bar{C}$ \\
\hline KAU055 & 22.5747 & 120.3353 & A900A & 16384 & 150.1 & $120.96^{*}$ & 225.6 & $\overline{\mathrm{D}}$ & $\mathrm{N} / \mathrm{A}$ & $\bar{E}$ \\
\hline KAU056 & 22.5538 & 120.3437 & $\mathrm{~A} 900 \mathrm{~A}$ & 16384 & 152 & $122.92^{*}$ & 225.6 & $\mathrm{D}$ & N/A & $\overline{\mathrm{E}}$ \\
\hline KAU057 & 22.6342 & 120.2633 & A900A & 16384 & 146.5 & 113.54 & 491.7 & $\mathrm{C}$ & $\mathrm{S}$ & $\mathrm{C}$ \\
\hline KAU062 & 22.6237 & 120.2843 & A900A & 16384 & 146.7 & 113.46 & 198 & $\mathrm{D}$ & $\mathrm{S}$ & $\mathrm{E}$ \\
\hline KAU063 & 22.9053 & 120.1732 & A900A & 16384 & 123.6 & $9 \overline{8.83}$ & 198 & $\bar{D}$ & $\mathrm{~S}$ & $\mathrm{E}$ \\
\hline KAU064 & 22.7852 & 120.2385 & A900A & 16384 & 132.2 & 100.51 & 198 & $\mathrm{D}$ & $\mathrm{S}$ & $E$ \\
\hline KAU066 & 22.7303 & 120.3412 & A900A & 16384 & 133.6 & 100.38 & 198 & $\overline{\mathrm{D}}$ & $\mathrm{S}$ & $\mathrm{E}$ \\
\hline KAU069 & 22.8873 & 120.6567 & A900A & 16384 & 108.7 & 70.71 & 524.6 & $\mathrm{C}$ & $\overline{\mathrm{R}}$ & $\bar{B}$ \\
\hline KAU073 & 22.5348 & 120.5362 & A900A & 16384 & 149.2 & 110.67 & 198 & $\mathrm{D}$ & $\bar{S}$ & $\mathrm{E}$ \\
\hline KAU074 & 22.574 & 120.5663 & $\overline{\mathrm{A} 900 \mathrm{~A}}$ & 16384 & 144.4 & 105.59 & 198.1 & $\mathrm{D}$ & $\bar{S}$ & $\mathrm{E}$ \\
\hline KAU075 & 22.4903 & 120.499 & A900A & 16384 & 154.8 & 116.57 & 198 & $\overline{\mathrm{D}}$ & $\mathrm{S}$ & $\mathrm{E}$ \\
\hline KAU077 & 22.747 & 120.7233 & A900A & 16384 & 123.5 & 82.96 & 603.9 & $\mathrm{C}$ & $\overline{\mathrm{R}}$ & $\mathrm{B}$ \\
\hline
\end{tabular}

Continued... 


\begin{tabular}{|c|c|c|c|c|c|c|c|c|c|c|}
\hline Station & Latitude & Longitude & Model & Count/G & EpiDist & Drup & $V_{s 30}$ & PEER & RSS & Geo \\
\hline KAU078 & $\begin{array}{l}22.7112 \\
\end{array}$ & 120.6412 & A900A & 16384 & 128.3 & 88.99 & 503.9 & $\mathrm{C}$ & $\mathrm{R}$ & $\mathrm{B}$ \\
\hline KAU081 & 22.0095 & 120.7367 & A900A & 16384 & 205.1 & 162.43 & 249.1 & $\bar{D}$ & $\mathbf{S}$ & $\bar{D}$ \\
\hline KAU083 & 22.5673 & 120.4475 & A900A & 16384 & 147.7 & 110.95 & 198 & $\mathrm{D}$ & $\mathbf{S}$ & $\mathrm{E}$ \\
\hline KAU085 & 22.8858 & 120.3222 & IDSA & 16384 & 118.4 & 86.54 & $2 \overline{260.8}$ & $\mathrm{D}$ & $\mathbf{R}$ & $\mathrm{D}$ \\
\hline KAU086 & 22.795 & 120.295 & IDSA & 16384 & 128.8 & 96.59 & 198 & $\bar{D}$ & $\mathrm{~S}$ & $\bar{E}$ \\
\hline KAU087 & 22.6128 & 120.3113 & IDSA & 16384 & 146.9 & 113.13 & 198 & $\mathrm{D}$ & $\bar{S}$ & $\mathbf{E}$ \\
\hline KAU088 & 22.6461 & 120.311 & IDSA & 16384 & 143.5 & 110.22 & 198 & $\bar{D}$ & $\bar{S}$ & $\mathbf{E}$ \\
\hline KAU089 & 22.4802 & 120.4028 & IDSA & 16384 & 158.1 & $129.26^{*}$ & 189.9 & $\mathrm{D}$ & N/A & $\mathbf{E}$ \\
\hline $\mathrm{NCU}$ & 24.97 & 121.1867 & A800 & 2048 & 129 & 80.44 & 492.2 & $\mathrm{C}$ & $\mathbf{R}$ & $?$ \\
\hline NSK & 24.6755 & 121.3583 & A800 & 2048 & 106.6 & 58.09 & 598.5 & $\mathrm{C}$ & $\mathbf{R}$ & $\mathrm{B}$ \\
\hline NST & $\begin{array}{l}24.6312 \\
\end{array}$ & 121.0005 & A800 & 2048 & 87.8 & 38.43 & 495.5 & $\mathrm{C}$ & $\mathrm{R}$ & $\mathrm{D}$ \\
\hline NSY & $\begin{array}{l}24.4162 \\
\end{array}$ & 120.7607 & A800 & 2048 & 61.7 & 13.15 & 599.6 & $\mathrm{C}$ & $\mathbf{R}$ & $\mathrm{C}$ \\
\hline PNG & 23.5672 & 119.5552 & A800 & 2048 & 131 & 110.31 & 409.9 & $\mathrm{C}$ & $\mathbf{R}$ & $\mathrm{B}$ \\
\hline SGS & 23.0817 & 120.5827 & A800 & 2048 & $8 \overline{9}$ & $60.29^{*}$ & $\mathrm{~N} / \mathrm{A}$ & $\mathrm{N} / \mathrm{A}$ & $\mathrm{N} / \mathrm{A}$ & B \\
\hline SSD & 22.746 & 120.632 & A800 & 2048 & 124.6 & 85.75 & 499.1 & $\mathrm{C}$ & $\mathrm{R}$ & $\mathrm{C}$ \\
\hline STY & 23.1625 & 120.7573 & A800 & 2048 & 77.4 & 40.49 & 596.6 & $\mathrm{C}$ & $\overline{\mathrm{R}}$ & $\mathrm{B}$ \\
\hline TAP & 25.0392 & 121.5225 & A800 & 2048 & 149.8 & 100.03 & 198 & $\bar{D}$ & $\mathrm{~S}$ & $\mathrm{E}$ \\
\hline TAP003 & 25.0863 & 121.45 & ETNA & $\overline{4194304}$ & 151 & 102.39 & 212.4 & $\mathrm{D}$ & $\mathrm{S}$ & $\bar{E}$ \\
\hline TAP005 & 25.1077 & 121.507 & ETNA & 4194304 & 155.7 & 106.87 & 198 & $\bar{D}$ & $\bar{S}$ & $\bar{E}$ \\
\hline TAP006 & 25.0947 & 121.5093 & ETNA & 4194304 & 154.5 & 105.66 & 198 & $\mathrm{D}$ & $\mathrm{S}$ & $E$ \\
\hline TAP007 & 25.0758 & 121.5083 & ETNA & 4194304 & 152.6 & 103.73 & 198 & $\overline{\mathrm{D}}$ & $\mathrm{S}$ & $\mathrm{E}$ \\
\hline TAP008 & 25.0758 & 121.5273 & ETNA & 4194304 & 153.5 & 104.52 & 198 & $\bar{D}$ & $\mathrm{~S}$ & $\mathrm{E}$ \\
\hline TAP010 & 25.0677 & 121.4813 & ETNA & 4194304 & 150.5 & 101.33 & 226.4 & $\mathrm{D}$ & $\mathrm{S}$ & $\mathrm{E}$ \\
\hline TAP012 & 25.0563 & 121.5078 & ETNA & 4194304 & 150.7 & 101.76 & 198 & $\mathrm{D}$ & $\mathrm{S}$ & $E$ \\
\hline TAP013 & 25.0572 & 121.5253 & ETNA & 4194304 & 151.6 & 102.59 & 198 & $\bar{D}$ & $\mathrm{~S}$ & $\mathrm{E}$ \\
\hline TAP014 & 25.0578 & 121.5442 & ETNA & $\overline{4194304}$ & 152.6 & 103.45 & 198 & $\bar{D}$ & $\bar{S}$ & $\overline{\mathrm{E}}$ \\
\hline TAP017 & 25.0528 & 121.448 & ETNA & 4194304 & 147.5 & 98.93 & 198 & $\mathrm{D}$ & $\mathrm{S}$ & $\mathrm{E}$ \\
\hline TAP020 & 25.0388 & 121.5263 & ETNA & 4194304 & 149.9 & 100.8 & 198 & $\mathrm{D}$ & $\mathrm{S}$ & $\mathrm{E}$ \\
\hline TAP021 & 25.0378 & 121.5432 & ETNA & 4194304 & 150.7 & 101.43 & 198 & $\bar{D}$ & $\mathrm{~S}$ & $\mathrm{E}$ \\
\hline TAP024 & 25.0203 & 121.4652 & ETNA & 4194304 & 145.1 & 96.39 & 195 & $\mathrm{D}$ & $\bar{S}$ & $\mathrm{D}$ \\
\hline TAP026 & 25.017 & 121.5027 & ETNA & 4194304 & 146.6 & 97.64 & 198 & $\bar{D}$ & $\mathrm{~S}$ & $\mathrm{E}$ \\
\hline TAP028 & 25.0045 & 121.5073 & ETNA & 4194304 & 145.6 & 96.6 & 227.2 & $\bar{D}$ & $\mathrm{~S}$ & $\mathrm{D}$ \\
\hline TAP032 & 25.0007 & 121.4748 & A900 & 16384 & 143.7 & 94.84 & 385 & $\mathrm{C}$ & $\mathrm{S}$ & $\overline{\mathrm{D}}$ \\
\hline TAP034 & 24.955 & 121.5303 & A900 & 16384 & 142.1 & 92.73 & 408.7 & $\mathrm{C}$ & $\mathrm{R}$ & $\mathrm{B}$ \\
\hline TAP035 & 24.9239 & 121.5399 & A900 & 16384 & 139.7 & 89.91 & 438.1 & $\mathrm{C}$ & $\mathrm{R}$ & $\mathrm{B}$ \\
\hline TAP036 & 24.9043 & 121.5447 & A900 & 16384 & 138.2 & 88.45 & 4887.2 & $\mathrm{C}$ & $\mathrm{S}$ & $\mathrm{B}$ \\
\hline ТAि041 & 25.183 & 121.4167 & A900 & 16384 & 159.3 & 110.9 & 225.6 & $\mathrm{D}$ & $\mathrm{S}$ & $\mathrm{D}$ \\
\hline TAP042 & 25.1255 & 121.4532 & A900 & 16384 & 155 & 106.48 & 225.3 & $\overline{\mathrm{D}}$ & $\mathrm{S}$ & $\mathrm{D}$ \\
\hline TAP043 & 24.9913 & 121.41 & A900 & 16384 & 139.7 & 91.19 & 234.7 & $\mathrm{D}$ & $\mathrm{R}$ & $\mathrm{D}$ \\
\hline TAP046 & 25.1037 & 121.7683 & A900 & 16384 & 169.2 & 118.34 & 410.9 & $\mathrm{C}$ & $\mathrm{S}$ & $\overline{\mathrm{D}}$ \\
\hline TAP047 & 24.9538 & 121.3375 & A900 & 16384 & 132.8 & 84.46 & 388.1 & $\mathrm{C}$ & $\mathrm{R}$ & $\mathrm{D}$ \\
\hline TAP049 & 25.148 & 121.4365 & A900 & 16384 & 156.6 & 108.1 & 419.5 & $\mathrm{C}$ & $\mathrm{S}$ & $\mathrm{D}$ \\
\hline TAP051 & 25.1008 & 121.4403 & A900 & 16384 & 152 & 103.46 & 403.2 & $\mathrm{C}$ & $\bar{R}$ & $\mathrm{~B}$ \\
\hline TAP052 & 25.0817 & 121.3828 & A900 & 16384 & 147.6 & 99.24 & 527.7 & $\mathrm{C}$ & $\mathbf{R}$ & $\mathrm{C}$ \\
\hline TAP053 & 24.959 & 121.5143 & A900 & 16384 & 141.7 & 92.42 & 394.7 & $\mathrm{C}$ & $\mathrm{R}$ & $\mathrm{D}$ \\
\hline TAP059 & 25.1565 & 121.6855 & A900 & 16384 & 169.3 & 119.3 & 478.2 & $\mathrm{C}$ & $\mathbf{R}$ & $\mathrm{B}$ \\
\hline ТÄР060 & 25.1572 & $1 \overline{21.7237}$ & A900 & 16384 & 171.5 & 121.15 & 439.6 & $\mathrm{C}$ & $\mathrm{S}$ & $\mathrm{B}$ \\
\hline TAP065 & 25.1488 & 121.7668 & A900A & 16384 & 173.1 & 122.48 & 1023.5 & $\mathrm{~B}$ & $\mathrm{~S}$ & $?$ \\
\hline TAP066 & 25.1865 & 121.5202 & A900A & 16384 & 164.1 & 115.34 & $\begin{array}{l}662.8 \\
\end{array}$ & $\mathrm{C}$ & $\bar{S}$ & $\mathrm{C}$ \\
\hline TAP067 & 24.9802 & 121.5802 & $\mathrm{~A} 900 \mathrm{~A}$ & 16384 & 147.2 & 97.39 & 552.9 & $\mathrm{C}$ & $\mathbf{R}$ & $\bar{B}$ \\
\hline TAP069 & 25.0082 & 121.9882 & A900A & 16384 & 175.2 & 123.57 & 519 & $\mathrm{C}$ & $\mathrm{S}$ & B \\
\hline TAP072 & 24.9913 & 121.65 & IDSA & 16384 & 152.1 & 101.74 & 529.5 & $\bar{C}$ & $\mathbf{R}$ & $\mathrm{B}$ \\
\hline TAP075 & 25.0288 & 121.728 & IDSA & 16384 & 160 & 109.27 & 552.5 & $\mathrm{C}$ & $\mathrm{S}$ & $\bar{B}$ \\
\hline TAP077 & 25.0643 & 121.8437 & IDSA & 16384 & 170.3 & 119 & 1022.8 & $\mathrm{~B}$ & $\mathrm{~S}$ & $\mathrm{D}$ \\
\hline TAP078 & 25.0354 & 121.8595 & IDSA & 16384 & 168.8 & 119.97 & 501.9 & $\mathrm{C}$ & $\mathrm{S}$ & $\mathrm{B}$ \\
\hline
\end{tabular}

Continued... 


\begin{tabular}{|c|c|c|c|c|c|c|c|c|c|c|}
\hline Station & Latitude & Longitude & Model & Count/G & EpiDist & Drup & $V_{s 30}$ & PEER & $\mathrm{RSS}$ & Geo \\
\hline TAP079 & 25.0237 & 121.906 & IDSA & 16384 & 170.9 & 119.36 & 413.2 & $\mathrm{C}$ & $\mathrm{S}$ & $\bar{B}$ \\
\hline TAP081 & 25.0182 & 121.9808 & IDSA & 16384 & 175.5 & 123.86 & 397.9 & $\mathrm{C}$ & $\mathrm{S}$ & $\bar{B}$ \\
\hline TAP083 & 25.2595 & 121.4938 & IDSA & 16384 & 170.2 & 121.7 & 404.1 & $\mathrm{C}$ & $\mathrm{S}$ & $\bar{D}$ \\
\hline TAP084 & 25.2252 & 121.6295 & IDSA & 16384 & 173 & 123.71 & 224.2 & $\overline{\mathrm{D}}$ & $\mathrm{S}$ & $\overline{\mathrm{D}}$ \\
\hline TAP086 & 24.9527 & 121.5677 & A900 & 16384 & 143.9 & 94.16 & 489.5 & $\mathrm{C}$ & $\mathrm{R}$ & $\mathrm{C}$ \\
\hline TAP087 & 25.1008 & 121.4177 & A900A & 16384 & 151 & 102.56 & 434.5 & $\mathrm{C}$ & $\mathrm{S}$ & $\mathrm{C}$ \\
\hline TAP088 & 25.0385 & 121.5762 & ETNA & 4194304 & 152.4 & $93.26^{*}$ & 418 & $\mathrm{C}$ & N/A & $\bar{D}$ \\
\hline TAP089 & 25.027 & 121.5595 & ETNA & 4194304 & 150.5 & $91.31^{*}$ & 440.2 & $\mathrm{C}$ & N/A & $\bar{D}$ \\
\hline TAP090 & 25.0573 & 121.5945 & ETNA & 4194304 & 155.2 & 105.56 & 324.4 & $\mathrm{D}$ & $\mathrm{S}$ & $\bar{E}$ \\
\hline TAP094 & 25.141 & 121.4758 & ETNA & 4194304 & 157.5 & 108.96 & 255.4 & $\mathrm{D}$ & $\mathrm{S}$ & $\overline{\mathrm{D}}$ \\
\hline TAP095 & 25.1353 & 121.4913 & ETNA & 4194304 & 157.7 & 109.01 & 198 & $\overline{\mathrm{D}}$ & $\bar{S}$ & $\bar{E}$ \\
\hline TAP097 & 25.0207 & 121.5299 & IDSA & 16384 & 148.3 & 99.17 & 198 & $\mathrm{D}$ & $\mathrm{S}$ & $E$ \\
\hline TAP098 & 25.103 & 121.5395 & ETNA & 4194304 & 156.8 & 107.75 & 457.6 & $\mathrm{C}$ & $\mathrm{S}$ & $\mathrm{C}$ \\
\hline TAP100 & 25.0351 & 121.5139 & ETNA & 4194304 & 148.9 & 100.13 & 198 & $\bar{D}$ & $\mathrm{~S}$ & $E$ \\
\hline TAP103 & 25.071 & 121.781 & A900A & 16384 & 167 & 116.02 & 429.5 & $\mathrm{C}$ & $\bar{R}$ & $\mathrm{C}$ \\
\hline TAP104 & 25.158 & 121.7202 & ETNA & 4194304 & 171.4 & $112.22^{*}$ & 396.1 & $\mathrm{C}$ & N/A & $\bar{B}$ \\
\hline TCU & 24.1475 & 120.676 & A800 & 2048 & 34.2 & 5.18 & 472.8 & C & $\mathrm{R}$ & $\overline{\mathrm{D}}$ \\
\hline TCU003 & $25 . \overline{0473}$ & 121.1357 & A900 & 16384 & 135.8 & 86.57 & 517.3 & $\mathrm{C}$ & $\mathrm{S}$ & $\bar{D}$ \\
\hline TCU006 & 24.9118 & 121.1405 & A900 & 16384 & 121.5 & 72.61 & 505.5 & $\mathrm{C}$ & $\mathrm{S}$ & $\overline{\mathrm{D}}$ \\
\hline TCU007 & 25.0015 & 121.3097 & A900 & 16384 & 136.6 & 88.2 & 462.8 & $\mathrm{C}$ & $\overline{\mathrm{S}}$ & $\overline{\mathrm{D}}$ \\
\hline TCU008 & 25.0092 & 121.2062 & A900 & 16384 & 133.8 & 85.09 & 457.6 & $\mathrm{C}$ & $\mathrm{S}$ & $\mathrm{C}$ \\
\hline TCU010 & 25.0003 & 121.1533 & A900 & 16384 & 131.3 & 82.27 & 469.9 & $\mathrm{C}$ & $\bar{S}$ & $\mathrm{C}$ \\
\hline TCU014 & 25.0463 & 121.3075 & A900 & 16384 & 141.1 & 92.7 & 473.4 & $\mathrm{C}$ & $\mathbf{R}$ & $\bar{D}$ \\
\hline TCU015 & 24.7572 & 120.9345 & A900 & 16384 & 100.3 & 49.81 & 416.3 & $\mathrm{C}$ & $\bar{S}$ & $\overline{\mathrm{C}}$ \\
\hline TCU017 & 24.7808 & 121.0068 & A900 & 16384 & 104.1 & 54.28 & 558.8 & $\mathrm{C}$ & $\mathrm{S}$ & $?$ \\
\hline TCU018 & 24.88 & 121.0535 & A900 & 16384 & 115.8 & 66.25 & 475 & $\mathrm{C}$ & $\mathbf{R}$ & $\mathrm{C}$ \\
\hline TCU026 & 24.7757 & 121.075 & A900 & 16384 & 105.2 & 56.12 & 472.9 & $\mathrm{C}$ & $\mathrm{R}$ & $\bar{D}$ \\
\hline TCU029 & 24.5588 & 120.7492 & A900 & 16384 & 77.5 & 28.05 & 410.9 & $\mathrm{C}$ & $\mathrm{R}$ & $\mathrm{C}$ \\
\hline TCU031 & 24.5615 & 120.701 & A900 & 16384 & 78.3 & 30.18 & 393.8 & $\mathrm{C}$ & $\mathrm{S}$ & $\bar{D}$ \\
\hline TCU033 & 24.6855 & 120.8623 & A900 & 16384 & 91.6 & 40.89 & 423.4 & $\mathrm{C}$ & $\bar{S}$ & $\mathrm{D}$ \\
\hline TCU034 & 24.639 & 120.8568 & A900 & 16384 & 86.4 & 35.69 & 392.1 & $\mathrm{C}$ & $\mathbf{R}$ & $\mathrm{C}$ \\
\hline TCU035 & 24.6157 & $\overline{120.7877}$ & A900 & 16384 & 83.7 & $31.1^{*}$ & 227.9 & $\overline{\mathrm{D}}$ & $\mathrm{N} / \mathrm{A}$ & $\overline{\mathrm{D}}$ \\
\hline TCU036 & 24.4488 & 120.6963 & A900 & 16384 & 66 & 19.84 & 489.3 & $\mathrm{C}$ & $\mathrm{S}$ & $\bar{D}$ \\
\hline TCU038 & 24.4912 & 120.663 & A900 & 16384 & 71.2 & 25.44 & 225.6 & $\mathrm{D}$ & $\mathrm{S}$ & $\overline{\mathrm{D}}$ \\
\hline TCU039 & 24.4917 & 120.7837 & A900 & 16384 & 70 & 19.9 & 540.7 & $\mathrm{C}$ & $\mathrm{S}$ & $\bar{C}$ \\
\hline TCU040 & 24.4497 & 120.6455 & A900 & 16384 & 67.1 & 22.08 & 362 & $\mathrm{C}$ & $\mathrm{S}$ & $\bar{E}$ \\
\hline TCU042 & 24.5542 & 120.8077 & A900 & 16384 & 76.9 & 26.32 & 412.6 & $\mathrm{C}$ & $\mathrm{R}$ & $\overline{\mathrm{D}}$ \\
\hline TCU045 & 24.5412 & 120.9137 & A900 & 16384 & 76.3 & 26 & 704.6 & $\mathrm{C}$ & $\mathrm{R}$ & $\mathrm{C}$ \\
\hline TCU046 & 24.4683 & 120.8543 & A900 & 16384 & 67.6 & 16.74 & 465.5 & $\bar{C}$ & $\mathrm{R}$ & $\bar{B}$ \\
\hline TCU047 & 24.6188 & 120.9387 & A900 & 16384 & 85.2 & 35 & 520.4 & $\mathrm{C}$ & $\bar{R}$ & $\bar{C}$ \\
\hline TCU048 & 24.18 & 120.5888 & A900 & 16384 & 41.4 & 13.55 & 508.5 & $\mathrm{C}$ & $\mathrm{S}$ & $\bar{C}$ \\
\hline TCU049 & 24.179 & 120.6902 & A900 & 16384 & 37 & 3.78 & 4887.3 & $\mathrm{C}$ & $\overline{\mathrm{R}}$ & $\overline{\mathrm{D}}$ \\
\hline TCU050 & 24.1815 & 120.6338 & A900 & 16384 & 39.4 & 9.51 & 462.5 & $\mathrm{C}$ & $\mathrm{S}$ & $\overline{\mathrm{D}}$ \\
\hline TCU051 & 24.1603 & 120.6518 & A900 & 16384 & 36.5 & 7.66 & 459.1 & $\mathrm{C}$ & $\bar{S}$ & $\bar{D}$ \\
\hline TCU052 & 24.198 & 120.7393 & A900 & 16384 & 37.9 & 0.66 & 393.2 & $\mathrm{C}$ & $\mathrm{S}$ & $\overline{\mathrm{D}}$ \\
\hline TCU053 & 24.1935 & 120.6688 & A900 & 16384 & 39.2 & 5.97 & 454.5 & $\mathrm{C}$ & $\mathrm{R}$ & $\bar{D}$ \\
\hline TCU054 & 24.1612 & 120.675 & A900 & 16384 & 35.7 & 5.3 & 460.7 & $\mathrm{C}$ & $\mathrm{S}$ & $\overline{\mathrm{D}}$ \\
\hline TCU055 & 24.1392 & 120.6643 & A900 & 16384 & 33.8 & 6.36 & 437.8 & $\mathrm{C}$ & $\mathrm{S}$ & $\overline{\mathrm{D}}$ \\
\hline TCU056 & 24.1588 & 120.6238 & A900 & 16384 & 37.6 & 10.5 & 429.6 & $\mathrm{C}$ & $\mathrm{S}$ & $\overline{\mathrm{D}}$ \\
\hline TCU057 & 24.1732 & 120.6107 & A900 & 16384 & 39.6 & 11.84 & 466.8 & $\bar{C}$ & $\mathrm{R}$ & $\bar{C}$ \\
\hline TCU059 & 24.2687 & 120.5637 & A900 & 16384 & 51.2 & 17.13 & 226.5 & $\bar{D}$ & $\mathrm{~S}$ & $\overline{\mathrm{D}}$ \\
\hline TCU060 & 24.2247 & 120.644 & A900 & 16384 & 43.3 & 8.53 & 490.1 & $\bar{C}$ & $\bar{S}$ & $\bar{D}$ \\
\hline TCU061 & 24.1355 & 120.549 & A900 & 16384 & 39.7 & 17.19 & 309.2 & $\mathrm{D}$ & $\overline{\mathrm{S}}$ & $\overline{\mathrm{D}}$ \\
\hline TCU063 & 24.1083 & 120.6158 & A900 & 16384 & 33.2 & 9.8 & 282.5 & $\bar{D}$ & $\mathrm{~S}$ & $\overline{\mathrm{D}}$ \\
\hline TCU064 & 24.3457 & 120.61 & A900 & 16384 & 57.1 & 16.62 & 345.2 & $\mathrm{D}$ & $\mathrm{S}$ & $\bar{D}$ \\
\hline
\end{tabular}

Continued... 


\begin{tabular}{|c|c|c|c|c|c|c|c|c|c|c|}
\hline Station & Latitude & Longitude & Model & Count/G & EpiDist & Drup & $V_{s 30}$ & PEER & RSS & Geo \\
\hline TCU065 & 24.0588 & 120.6912 & A900 & 16384 & 24.6 & 0.59 & 305.9 & $\bar{D}$ & $\mathrm{R}$ & $\bar{D}$ \\
\hline TCU067 & 24.0912 & 120.72 & A900 & 16384 & 26.8 & 0.64 & 433.6 & $\mathrm{C}$ & $\mathrm{S}$ & $\mathrm{D}$ \\
\hline TCU068 & 24.2772 & 120.7658 & A900 & 16384 & 46.3 & 0.32 & 487.3 & $\mathrm{C}$ & $\mathrm{S}$ & $\mathrm{D}$ \\
\hline TCU070 & 24.196 & 120.5403 & A900 & 16384 & 45.6 & 19.02 & 401.3 & $\mathrm{C}$ & $\mathrm{S}$ & $\mathrm{C}$ \\
\hline TCU071 & 23.9855 & 120.7883 & A900 & 16384 & 13.9 & 5.31 & 624.8 & $\mathrm{C}$ & $\mathbf{R}$ & $\bar{D}$ \\
\hline TCU072 & 24.0407 & 120.8488 & A900 & 16384 & 20.6 & 7.03 & $\overline{468.1}$ & $\mathrm{C}$ & $\mathbf{R}$ & $\overline{\mathrm{D}}$ \\
\hline TCU074 & 23.9622 & 120.9618 & A900 & 16384 & 20 & 13.46 & 549.4 & $\bar{C}$ & $\overline{\mathrm{R}}$ & $\overline{\mathrm{D}}$ \\
\hline TCU075 & 23.9827 & 120.6778 & A900 & 16384 & 18.4 & 0.91 & 573 & $\mathrm{C}$ & $\mathrm{S}$ & $\mathrm{D}$ \\
\hline TCŨ076 & 23.9077 & 120.6757 & A900 & 16384 & 13.7 & 2.76 & 615 & $\mathrm{C}$ & $\bar{R}$ & $\mathrm{D}$ \\
\hline TCU078 & 23.812 & 120.8455 & A900 & 16384 & 7.1 & 8.2 & 443 & $\mathrm{C}$ & $\mathbf{R}$ & $\bar{D}$ \\
\hline TCU079 & 23.8395 & 120.8942 & A900 & 16384 & 9.9 & 10.97 & 364 & $\overline{\mathrm{C}}$ & $\mathbf{R}$ & $\mathrm{D}$ \\
\hline TCU081 & 24.8022 & 120.9695 & A900A & 16384 & 105.7 & 55.49 & 291.2 & $\bar{D}$ & $\mathrm{~S}$ & $\bar{D}$ \\
\hline TCU082 & 24.1475 & 120.676 & A900A & 16384 & 34.2 & 5.18 & 472.8 & $\mathrm{C}$ & $\mathrm{S}$ & $\mathrm{D}$ \\
\hline TCU083 & 24.97 & 121.1867 & A900A & 16384 & 129 & 80.32 & 490.6 & $\mathrm{C}$ & $\bar{R}$ & $?$ \\
\hline TCU084 & 23.883 & 120.8998 & A900A & 16384 & 10.5 & 11.24 & 607.2 & $\mathrm{C}$ & $\mathrm{R}$ & $?$ \\
\hline TCU085 & 24.6755 & 121.3583 & A900A & 16384 & 106.6 & 58.09 & 598.5 & $\mathrm{C}$ & $\overline{\mathrm{R}}$ & $\bar{B}$ \\
\hline TCU087 & 24.3482 & 120.7733 & A900A & 16384 & 54.1 & 7 & 530.3 & $\mathrm{C}$ & $\mathrm{R}$ & $\mathrm{C}$ \\
\hline TCU088 & 24.2533 & 121.1758 & A900A & 16384 & 58 & 18.16 & 616 & $\mathrm{C}$ & $\mathrm{S}$ & B \\
\hline TCU089 & 23.9037 & $120 . \overline{8} 565$ & A900A & 16384 & 7.5 & 8.88 & 599.5 & $\mathrm{C}$ & $\bar{R}$ & $\mathrm{C}$ \\
\hline TCU092 & 25.0117 & 121.2792 & A900 & 16384 & 136.5 & 88.07 & $\begin{array}{l}477.8 \\
\end{array}$ & $\mathrm{C}$ & $\mathrm{S}$ & $\mathrm{D}$ \\
\hline TCU094 & 24.7695 & 121.0488 & A900 & 16384 & 103.8 & 54.53 & 589.8 & $\mathrm{C}$ & $\mathrm{S}$ & $\mathrm{C}$ \\
\hline TCU095 & 24.6917 & 121.0135 & A900 & 16384 & 94.6 & 45.18 & 446.6 & $\mathrm{C}$ & $\overline{\mathrm{R}}$ & $\mathrm{C}$ \\
\hline TCU096 & 24.7957 & 120.9558 & A900 & 16384 & 104.8 & 54.46 & 399.4 & $\overline{\mathrm{C}}$ & $\mathrm{S}$ & $\mathrm{D}$ \\
\hline TCU098 & 24.7433 & 120.899 & A900 & 16384 & 98.3 & 47.67 & 226 & $\mathrm{D}$ & $\mathrm{R}$ & $\mathrm{D}$ \\
\hline TCU100 & 24.1858 & 120.6153 & A900 & 16384 & 40.6 & 11.39 & 466.8 & $\bar{C}$ & $\overline{\mathrm{R}}$ & $\mathrm{C}$ \\
\hline TCU101 & 24.242 & 120.7092 & A900 & 16384 & 43.3 & 2.13 & 499.7 & $\mathrm{C}$ & $\mathrm{S}$ & $\mathrm{D}$ \\
\hline TCU102 & 24.2493 & 120.7208 & A900 & 16384 & 43.8 & 1.51 & 714.3 & $\mathrm{C}$ & $\mathrm{S}$ & $\bar{D}$ \\
\hline TCU103 & 24.3098 & 120.7072 & A900 & 16384 & 50.7 & 6.1 & 494.1 & $\mathrm{C}$ & $\mathrm{S}$ & $\bar{D}$ \\
\hline TCU104 & 24.2455 & 120.6018 & A900 & 16384 & 47.2 & 12.89 & 513.9 & $\mathrm{C}$ & $\overline{\mathrm{R}}$ & $\mathrm{C}$ \\
\hline TCU105 & 24.239 & 120.559 & A900 & 16384 & 48.6 & 17.18 & 395.6 & $\mathrm{C}$ & $\mathrm{R}$ & $\mathrm{C}$ \\
\hline TCU106 & 24.0833 & 120.5518 & A900A & 16384 & 35.3 & 14.99 & 391.2 & $\mathrm{C}$ & $\mathrm{S}$ & $\mathrm{D}$ \\
\hline TCU107 & 24.0727 & 120.5402 & A900 & 16384 & 35.4 & 16.01 & 390.4 & $\bar{C}$ & $\bar{S}$ & $\bar{D}$ \\
\hline TCU109 & 24.0848 & 120.5713 & A900 & 16384 & 34 & 13.08 & 406.6 & $\mathrm{C}$ & $\mathrm{S}$ & $\mathrm{D}$ \\
\hline TCU110 & 23.9622 & 120.5695 & A900 & 16384 & 26 & 11.6 & 212.7 & $\bar{D}$ & $\mathrm{~S}$ & $\mathrm{E}$ \\
\hline TCU111 & 24.1137 & 120.4872 & A900 & 16384 & 42.4 & 22.14 & 237.5 & $\mathrm{D}$ & $\mathrm{S}$ & $\mathrm{E}$ \\
\hline TCU112 & 24.0557 & 120.424 & A900 & 16384 & 43.9 & 27.5 & 198 & $\overline{\mathrm{D}}$ & $\mathrm{S}$ & $\mathrm{E}$ \\
\hline TCU113 & 23.8928 & 120.3865 & A900 & 16384 & 42.2 & 31.07 & 230.3 & $\overline{\mathrm{D}}$ & $\overline{\mathrm{R}}$ & $\mathrm{E}$ \\
\hline TCU115 & 23.9595 & 120.4693 & A900 & 16384 & 35.4 & 21.78 & 215.3 & $\mathrm{D}$ & $\mathrm{R}$ & $\mathrm{E}$ \\
\hline TCU116 & 23.8568 & 120.5803 & A900 & 16384 & 22.3 & 12.4 & 493.1 & $\mathrm{C}$ & $\bar{S}$ & $\bar{E}$ \\
\hline TCU117 & 24.1335 & 120.4598 & A900 & 16384 & 45.9 & 25.44 & 198 & $\mathrm{D}$ & $\mathrm{S}$ & $\mathrm{E}$ \\
\hline TCU118 & 24.0027 & 120.4235 & A900 & 16384 & 41.4 & 26.84 & 198 & $\bar{D}$ & $\bar{S}$ & $\mathbf{E}$ \\
\hline TCU119 & 23.9242 & 120.3122 & A900 & 16384 & 50.1 & 37.95 & 198 & $\mathrm{D}$ & $\mathbf{R}$ & $E$ \\
\hline TCU120 & 23.9803 & 120.613 & A900 & 16384 & 23.2 & $7 . \overline{41}$ & 459.3 & $\mathrm{C}$ & $\mathrm{S}$ & $\mathrm{C}$ \\
\hline TCU122 & 23.8128 & 120.6097 & A900 & 16384 & 20 & 9.35 & 475.5 & $\mathrm{C}$ & $\mathbf{R}$ & $\mathrm{D}$ \\
\hline TCU123 & 24.0187 & 120.5437 & A900 & 16384 & 31.4 & 14.93 & 236.6 & $\mathrm{D}$ & $\mathrm{S}$ & $\mathrm{D}$ \\
\hline TCU128 & 24.4162 & 120.7607 & A900A & 16384 & 61.7 & 13.15 & 599.6 & $\mathrm{C}$ & $S$ & $\mathrm{C}$ \\
\hline TCU129 & 23.8783 & 120.6843 & A900A & 16384 & 11.9 & 1.84 & 664.4 & $\mathrm{C}$ & $\overline{\mathbf{R}}$ & $\mathrm{D}$ \\
\hline TCU131 & 24.5673 & 120.8165 & A900A & 16384 & $\begin{array}{l}78.3 \\
\end{array}$ & $26.15^{*}$ & 361.9 & $\mathrm{C}$ & $\mathrm{N} / \mathrm{A}$ & $\bar{D}$ \\
\hline TCU136 & 24.2603 & 120.6518 & IDS & 16384 & 46.8 & 8.29 & 509.2 & $\mathrm{C}$ & $\mathrm{S}$ & $\mathrm{C}$ \\
\hline TCU138 & 23.9223 & 120.5955 & IDSA & 16384 & 21.9 & 9.79 & 652.8 & $\mathrm{C}$ & $\mathbf{R}$ & $\bar{D}$ \\
\hline TCU140 & 23.9578 & 120.3593 & IDSA & 16384 & 46.1 & 32.97 & 198 & $\mathrm{D}$ & $\mathbf{R}$ & $\mathbf{E}$ \\
\hline TCU141 & 23.8338 & 120.464 & IDSA & 16384 & 34.3 & 24.21 & 206.5 & $\mathrm{D}$ & $\mathrm{S}$ & $\mathrm{E}$ \\
\hline TCU145 & 23.98 & 120.3368 & IDSA & 16384 & 48.9 & 35.34 & 198 & $\mathrm{D}$ & $\mathrm{R}$ & $\mathrm{E}$ \\
\hline TCU147 & 24.8592 & 121.2475 & A900 & 16384 & 119.6 & 71.27 & 537.9 & $\mathrm{C}$ & $\mathrm{S}$ & $\mathrm{N} / \mathrm{A}$ \\
\hline TTN & 22.754 & 121.1465 & A800 & 2048 & 127.6 & 83.31 & 228 & $\mathrm{D}$ & $\mathrm{S}$ & $\mathrm{D}$ \\
\hline
\end{tabular}

Continued... 


\begin{tabular}{|c|c|c|c|c|c|c|c|c|c|c|}
\hline Station & Latitude & Longitude & Model & Count/G & EpiDist & Drup & $V_{s 30}$ & PEER & RSS & Geo \\
\hline TTN001 & 23.3178 & 121.4425 & IDS & 16384 & 89 & 56.56 & 412.6 & $\mathrm{C}$ & $\mathrm{S}$ & $\mathrm{D}$ \\
\hline TTN002 & 22.9738 & 121.2968 & IDS & 16384 & 110.6 & 68.71 & 413 & $\mathrm{C}$ & $\mathrm{R}$ & $\mathrm{D}$ \\
\hline TTN003 & 22.6178 & 120.9975 & IDS & 16384 & 139.1 & 94.99 & 255.4 & $\mathrm{D}$ & $\mathrm{S}$ & $\mathrm{D}$ \\
\hline TTN004 & 22.9102 & 121.1287 & IDS & 16384 & 110.5 & 66.87 & 487.7 & $\mathrm{C}$ & $\mathrm{S}$ & $\mathrm{D}$ \\
\hline TTN007 & 22.7647 & 121.1427 & A900 & 16384 & 126.3 & 82.1 & 234.7 & $\mathrm{D}$ & $\mathrm{S}$ & $\bar{D}$ \\
\hline TTN008 & 22.7602 & 121.1517 & A900 & 16384 & 127 & 82.83 & 226.5 & $\bar{D}$ & $\mathrm{~S}$ & $\overline{\mathrm{D}}$ \\
\hline TTN009 & 22.7488 & 121.1442 & A900 & 16384 & 128 & 83.78 & 226.5 & $\mathrm{D}$ & $\mathrm{S}$ & $\mathrm{D}$ \\
\hline TTN010 & 22.74 & $1 \overline{21.1135}$ & A900 & 16384 & 128.2 & 83.88 & 241 & $\bar{D}$ & $\overline{\mathrm{S}}$ & $\bar{D}$ \\
\hline TTN012 & 22.7662 & 121.133 & $\mathrm{~A} 900$ & 16384 & 125.9 & 81.67 & 243.5 & $\mathrm{D}$ & $\mathrm{S}$ & $\mathrm{D}$ \\
\hline TTN014 & 23.0992 & 121.3653 & $\overline{\mathrm{A}} 900 \mathrm{~A}$ & 16384 & 102.2 & 63.53 & 304.7 & $\mathrm{D}$ & $\mathrm{S}$ & $\mathrm{D}$ \\
\hline TTN015 & 22.754 & 121.1465 & A900A & 16384 & 127.6 & 83.31 & 228 & $\bar{D}$ & $\mathrm{~S}$ & $\bar{D}$ \\
\hline TTN018 & 22.8207 & 121.0717 & A900A & 16384 & 118.5 & $74 . \overline{45}$ & 516.2 & $\mathrm{C}$ & $\mathbf{R}$ & $\mathrm{C}$ \\
\hline TTN020 & 23.1268 & 121.2057 & A900A & 16384 & 91.2 & 50.69 & 502.7 & $\mathrm{C}$ & $\mathbf{R}$ & $\mathrm{D}$ \\
\hline TTN022 & 23.0973 & 121.2105 & $\mathrm{~A} 900 \mathrm{~A}$ & 16384 & 94.4 & 53.34 & 502.6 & $\mathrm{C}$ & $S$ & $\mathrm{D}$ \\
\hline TTN023 & 23.053 & 121.1557 & A900A & 16384 & 96.5 & 54.29 & 500.9 & $\mathrm{C}$ & $\mathrm{S}$ & $\mathrm{D}$ \\
\hline TTN024 & 22.9725 & 121.1083 & A900A & 16384 & 103.3 & 60.01 & 562.8 & $\mathrm{C}$ & $\mathbf{R}$ & B \\
\hline TTN025 & 22.9045 & 121.072 & A900A & 16384 & 109.5 & 65.79 & 531.7 & $\mathrm{C}$ & $\mathbf{R}$ & $\mathrm{C}$ \\
\hline TTN026 & 22.863 & 121.083 & $\mathrm{~A} 900 \mathrm{~A}$ & 16384 & 114.2 & 70.33 & 525.4 & $\mathrm{C}$ & $\overline{\mathbf{R}}$ & $\mathrm{C}$ \\
\hline TTN027 & 22.8078 & 121.086 & A900A & 16384 & 120.2 & 76.13 & 495.9 & $\bar{C}$ & $\mathrm{~S}$ & $\mathrm{D}$ \\
\hline TTN028 & 22.779 & 121.0543 & A900A & 16384 & 122.6 & 78.48 & 486.7 & $\mathrm{C}$ & $\mathrm{R}$ & $\mathrm{B}$ \\
\hline TTN031 & 23.3563 & 121.4603 & A900A & 16384 & 87.5 & 56.3 & 340.9 & $\mathrm{D}$ & $\mathrm{R}$ & $\mathrm{D}$ \\
\hline TTN032 & 23.2462 & 121.4055 & A900A & 16384 & 91.9 & 57.65 & 406.6 & $\mathrm{C}$ & $\mathrm{S}$ & $\mathrm{D}$ \\
\hline TTN033 & 23.193 & 121.3878 & A900A & 16384 & 95.2 & 59.43 & 225.6 & $\mathrm{D}$ & $\mathrm{S}$ & $\bar{D}$ \\
\hline TTN036 & 22.7988 & 121.1855 & A900A & 16384 & 124 & 80 & 398.5 & $\mathrm{C}$ & $\mathrm{S}$ & $\mathrm{C}$ \\
\hline TTN040 & 23.1512 & 121.198 & IDS & 16384 & 88.5 & 48.33 & 533.9 & $\mathrm{C}$ & $\overline{\mathbf{R}}$ & $\bar{B}$ \\
\hline TTN041 & 23.1342 & 121.1177 & IDS & 16384 & 86.7 & 45.35 & 418.2 & $\mathrm{C}$ & $\mathbf{R}$ & B \\
\hline TTN042 & 23.0007 & 121.2767 & IDS & 16384 & 107 & 65.25 & 443.7 & $\mathrm{C}$ & $\mathrm{S}$ & $\mathrm{D}$ \\
\hline TTN044 & 23.0068 & 121.1655 & IDS & 16384 & 101.7 & 58.97 & 517.6 & $\mathrm{C}$ & $\mathbf{R}$ & $\mathrm{C}$ \\
\hline TTN045 & 22.9757 & 121.1478 & IDS & 16384 & 104.2 & 61.16 & 498.3 & $\mathrm{C}$ & $\mathrm{S}$ & $\mathrm{D}$ \\
\hline TTN046 & 22.9658 & $121 . \overline{3} \overline{2}$ & IDS & 16384 & 108.5 & 65.9 & 512.9 & $\mathrm{C}$ & $\overline{\mathbf{R}}$ & $\mathrm{C}$ \\
\hline TTN048 & 22.773 & 121.0827 & IDS & 16384 & 123.9 & 79.69 & 451.1 & $\mathrm{C}$ & $\mathrm{S}$ & $\mathrm{D}$ \\
\hline TTN050 & 22.674 & $121.0 \overline{29} \overline{3}$ & IDS & 16384 & 133.5 & 89.28 & 388.1 & $\mathrm{C}$ & $\overline{\mathrm{R}}$ & $\overline{\mathrm{D}}$ \\
\hline TTN051 & 23.1887 & 121.0168 & A900A & 16384 & 77.6 & 36.7 & 608.8 & $\mathrm{C}$ & $\mathrm{R}$ & $\mathrm{N} / \mathrm{A}$ \\
\hline WGK & 23.6862 & 120.5622 & A800 & 2048 & 30.9 & 9.96 & 258.9 & $\mathrm{D}$ & $\mathrm{S}$ & $\mathrm{D}$ \\
\hline WNT & 23.8783 & 120.6843 & A800 & 2048 & 11.9 & 1.84 & 664.4 & $\mathrm{C}$ & $\mathrm{R}$ & $\mathrm{D}$ \\
\hline WSF & 23.638 & 120.2217 & A800 & 2048 & 63.8 & 42.16 & 169.8 & $\mathrm{E}$ & $\mathrm{S}$ & $\mathrm{E}$ \\
\hline WTC & 23.8635 & 120.2812 & A800 & 2048 & 52.8 & 42.25 & 198 & $\overline{\mathrm{D}}$ & $\mathrm{S}$ & $\mathrm{E}$ \\
\hline
\end{tabular}

NOTES:

- Station: Accelerograph's station name.

- Latitude: Station latitude in degrees North (Lee et al., 2001b).

- Longitude: Station longitude in degrees East (Lee et al., 2001b).

- Model: Instrument model of the accelerograph (Lee et al., 2001b).

- Count/G: Factor to convert acceleration data in digital counts to G (Lee et al., 2001b).

- EpiDist: Distance (km) from Chi-Chi epicenter (23.8603 deg N, $120.7995 \mathrm{deg}$ E) (Lee et al., 2001b).

- Drup: Closest distance $(\mathrm{km})$ to the rupture surface (PEER, 2005); the distance with star is from Lee et al. (2001b). 
- $V_{s 30}$ : Shear wave velocity at top 30 meters (PEER, 2005).

- PEER: Site classification based on the $V_{s 30}$ from PEER (2005).

- RSS: Site classification based on the Response Spectral Shape method by Phung et al. (2004). "R" means rock sites and "S" means soil sites.

- Geo: Site classification mainly based on the geological and geomorphologic data by (Lee et al., 2001a).

- "B", "C", "D" and "E" are the site class according to NEHRP classification scheme;

- "?" means questionable sites while "N/A" means no information available. 
- Envelope calculation

In order to better identify the onset of $\mathrm{P}$-wave and S-wave phases, the envelope function is calculated for every record. The envelope function $E(t)$ of a seismogram $s(t)$ is defined as the absolute value of the complex envelope function in Equation 3.1

$$
E(t)=|s(t)-i H[s(t)]|
$$

where $H[s(t)]$ is the Hilbert transformation of the function $s(t)$ (Pacor et al., 2005). According to Bracewell (2000), the Hilbert transformation of a realvalued function $s(t)$ is defined in Equation 3.2

$$
\begin{aligned}
H[s(t)] & =\frac{1}{\pi} \int_{-\infty}^{\infty} \frac{s\left(t^{\prime}\right) d t^{\prime}}{t^{\prime}-t} \\
& =-\frac{1}{\pi t} * s(t)
\end{aligned}
$$

where ${ }^{*}$ ' denotes convolution operator.

The envelope function of $s(t)$ which we denote as $E(t)$ can be represented in Equation 3.4:

$$
E(t)=|s(t)-i H[s(t)]|=\sqrt{s^{2}(t)+H^{2}[s(t)]}
$$

- Windowing the Shear Wave Signal

Because the shear waves contribute most energy to the signal, in engineering seismology the portion of the seismogram we are interested in is the shear wave window. The simulated waveform from EXSIM is also the shear wave window. Thus, the definition of the shear wave window is important. The arrival time of the $\mathrm{P}$ wave has already been picked by Lee et al. (2001b) for each record. 
First, the start and end time of shear wave window are picked visually with the assistance of the calculated envelope of the seismogram. Figure 3.3 presents a sample seismogram for station ALS. In order to identify the ending of the shear wave window more accurately, I use $90 \%$ of the whole seismic record energy defined as $E=\frac{1}{2} \cdot|\mathrm{amp}|^{2}$ as a criteria to cut the extra noise signal. If the calculated time ( $90 \%$ energy of the total seismic record) is earlier than the ending time picked visually, it is then used as the ending time of the shear wave window. Otherwise, the ending time of the record picked visually is kept. The defined shear wave window is then used to compute the duration function later in Chapter 4.

- Remove Linear Trend and DC

The baseline of the recorded seismogram may move upward or downward in the latter part of the seismogram. Correction of records for any baseline or linear trend has been done by Lee et al. (2001b).

- Taper Function

In order to decrease the signal leakage (Gibbs phenomenon) when the Fourier transformation is applied on the windowed seismogram, a taper function is applied on the windowed seismogram. Generally, there are several commonly used taper functions available, such as: Hanning and Hamming windows, shown in Figure 3.4. However, the implementation of these window functions may lead to loss of energy since they cut off some part of the seismogram. To avoid this, a simple boxcar taper window (shown in the Figure 3.4 as well) is used for all of the data, in which $5 \%$ of the signal is tapered at each end.

- Instrument Response 
Accelerographs generally have flat response in the frequency range from 0 to 50 $\mathrm{Hz}$. Because the frequency range of this research is from $0.1 \mathrm{~Hz}$ to $20 \mathrm{~Hz}$, there is no need for instrument response correction, other than calibration to the flat response level.

- Output

The corrected seismic data are transformed to the frequency domain using Fast Fourier transformation. Since there are only two types of sampling rate for the seismic instrumentation of the TSMIP (200 samples/sec and 250 samples/sec), the lowest Nyquist frequency can reach up to $100 \mathrm{~Hz}$, which extends well beyond our range of interest $(0.1 \mathrm{~Hz}-20 \mathrm{~Hz})$. The computed Fourier spectrum is then $\log$ smoothed for later analysis (attenuation regression, kappa calculation and $\mathrm{H} / \mathrm{V}$ ratio computation). Twenty four log-spaced points have been generated between $0.1 \mathrm{~Hz}$ and $20 \mathrm{~Hz}$; the range between two contiguous points is treated as one frequency bin. The Fourier amplitude acceleration spectrum within each bin is averaged to represent the Fourier amplitude for such bin. $5 \%$ damped pseudo acceleration spectra (PSA) are calculated as well for all records. 


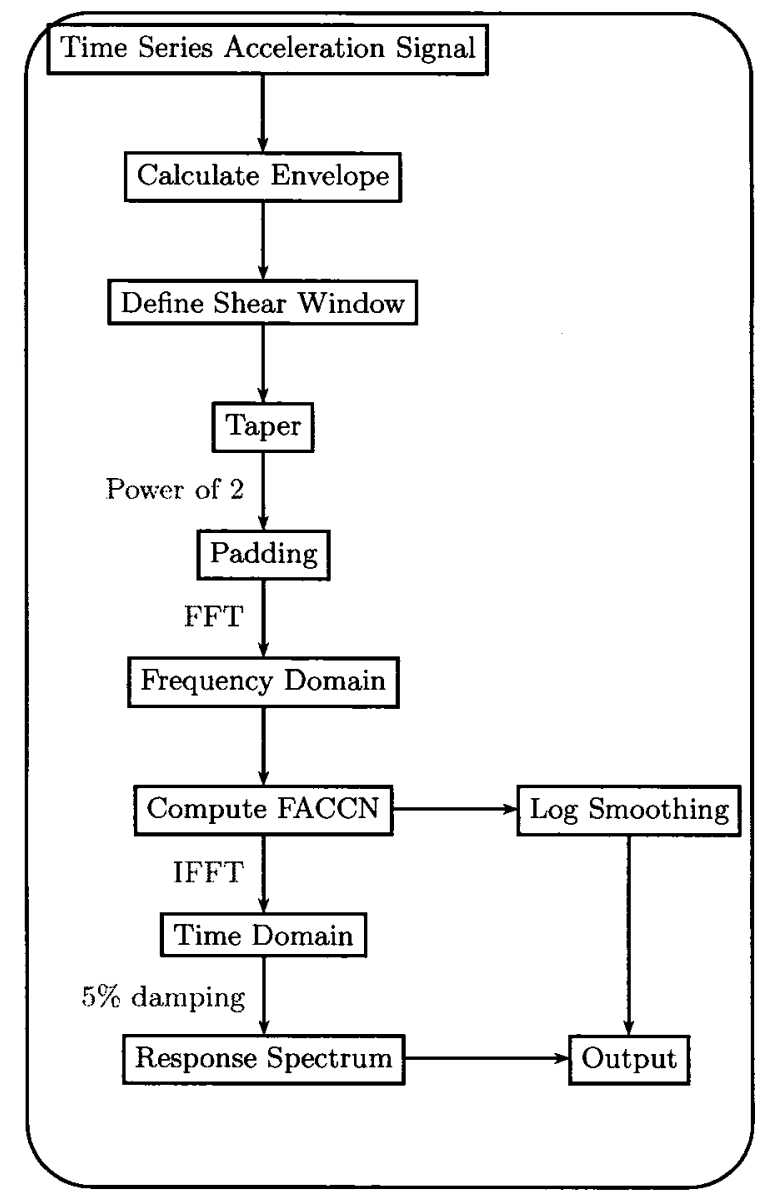

Figure 3.1. Flow chart for earthquake data processing 


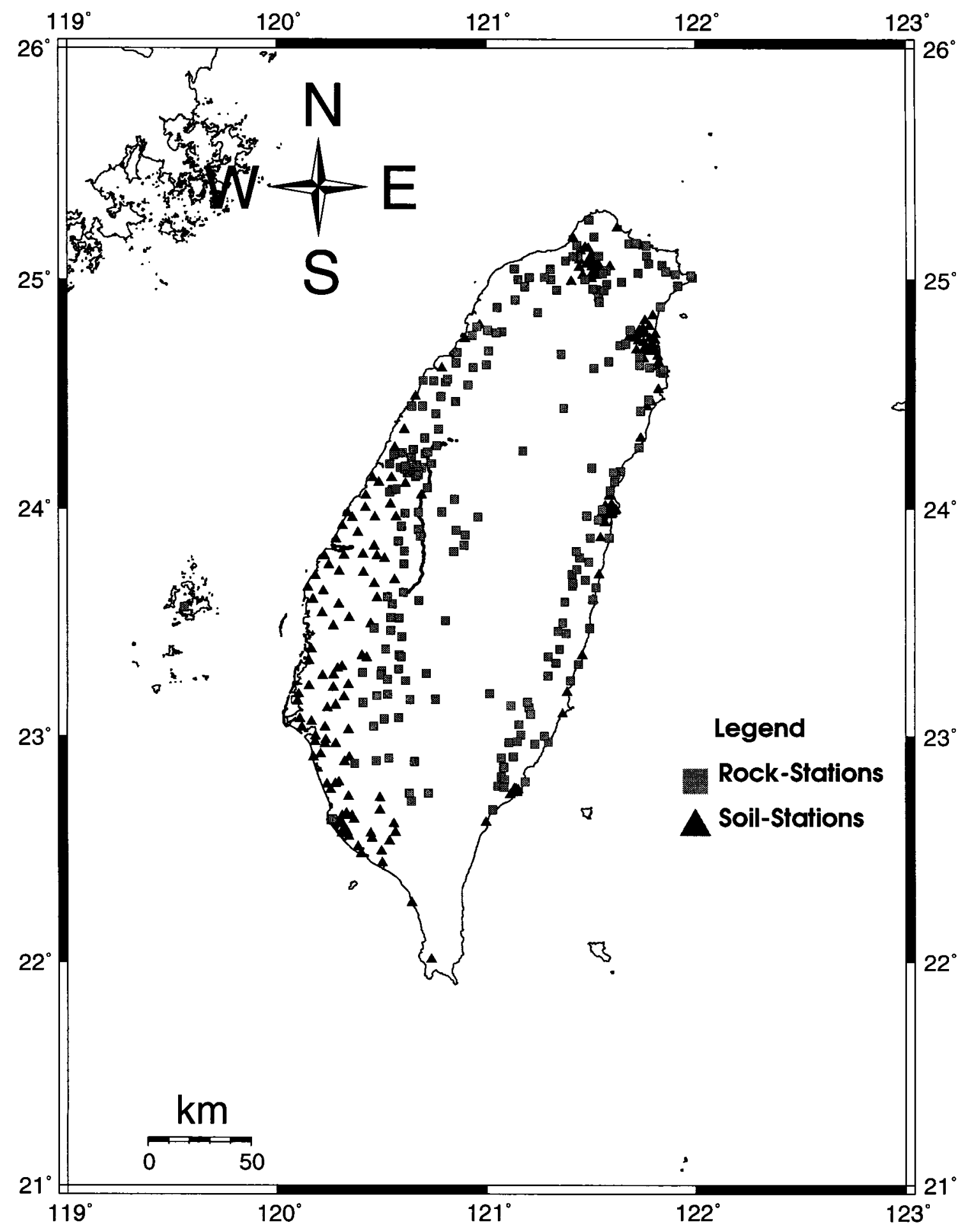

GM7 2005 Dec 01 02:07:15

Figure 3.2. Stations used in this study; the square represents the rock station while the up-triangle represents the soil station. The line shows the Chi-Chi surface rupture. 

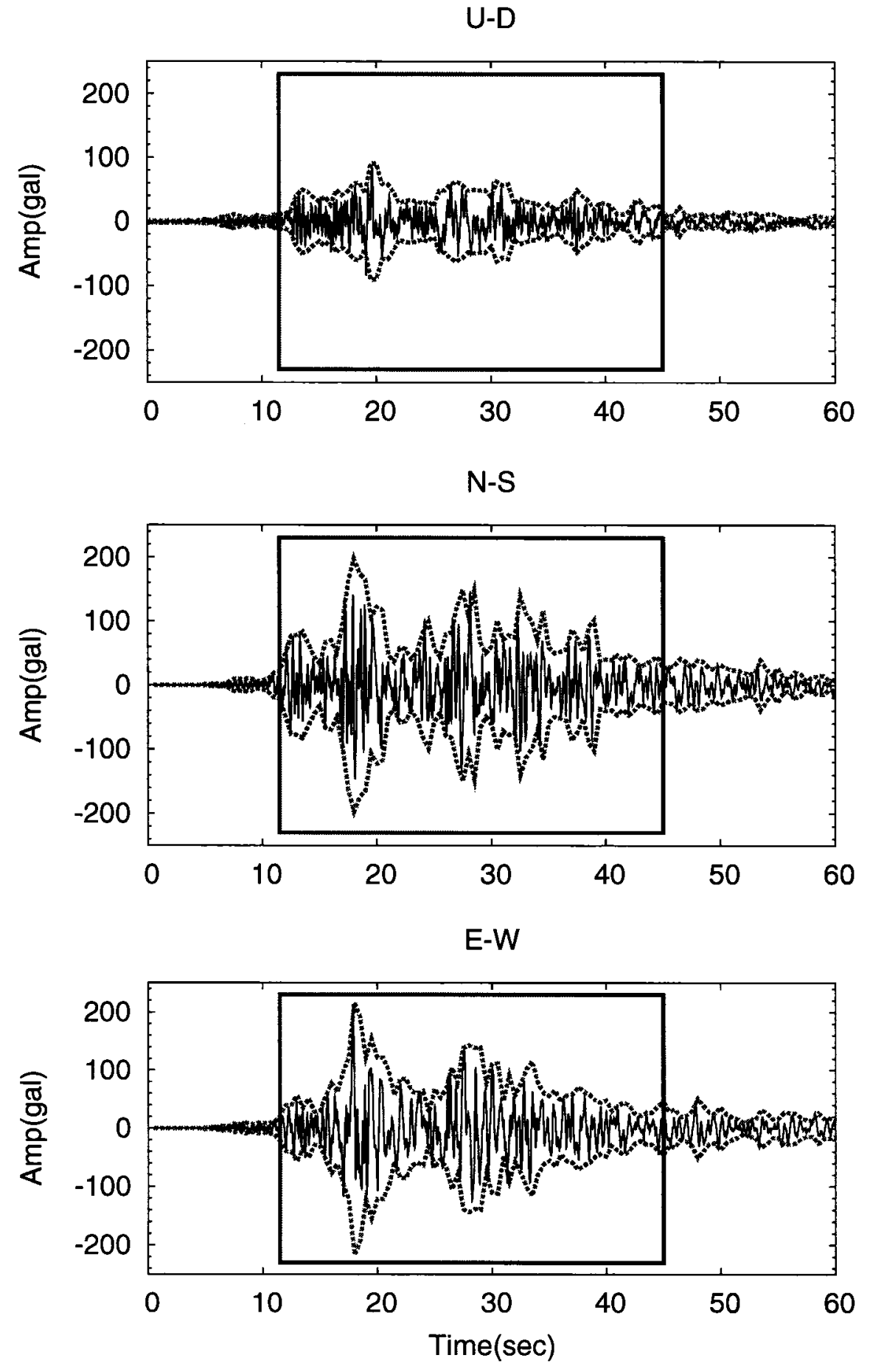

Figure 3.3. Sample seismogram with envelope function (dotted lines) and defined shear wave window (box) 
Taper Windows

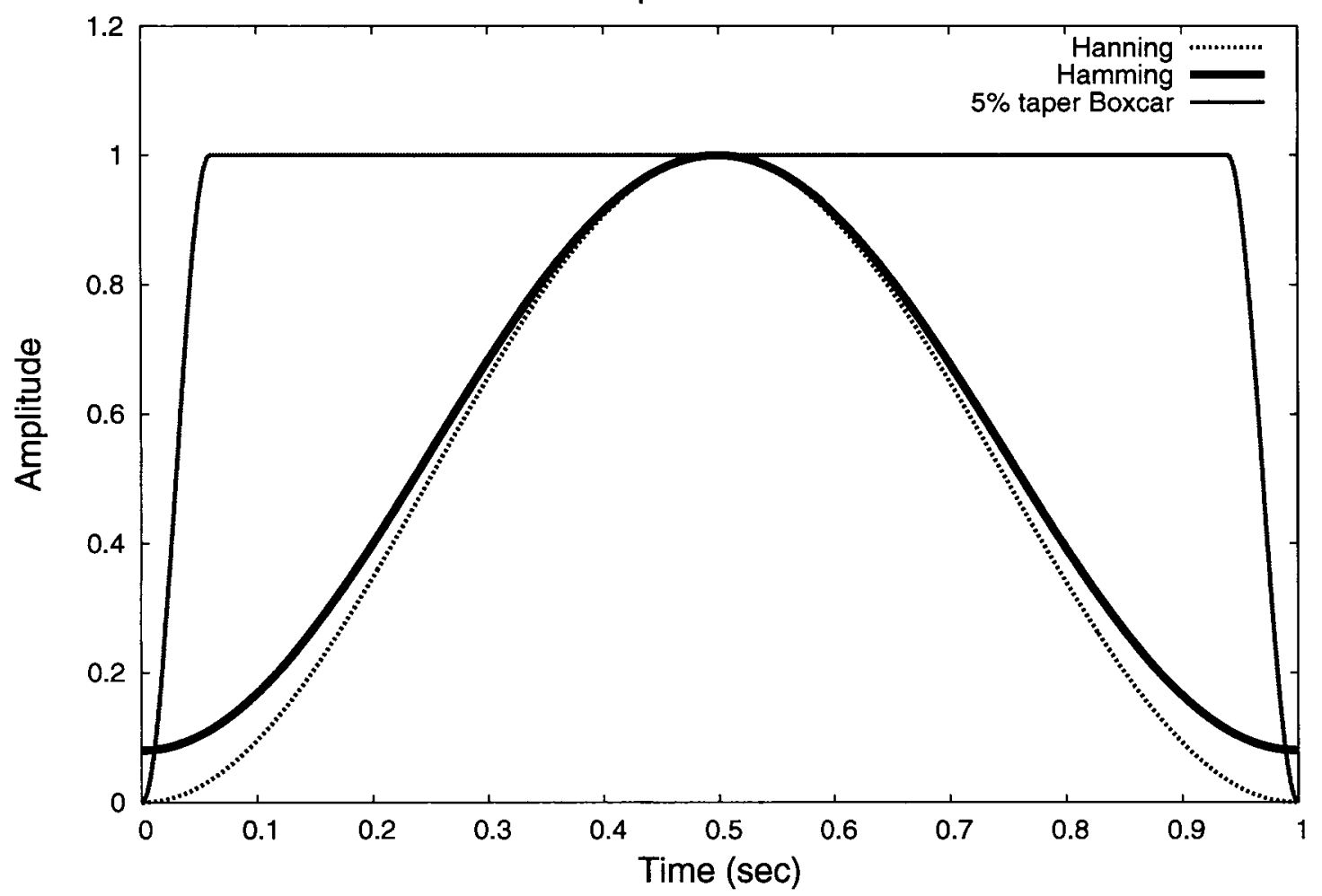

Figure 3.4. Commonly used taper functions for data processing 


\section{Chapter 4}

\section{SEISMIC PARAMETERS OF THE CHI-CHI MAINSHOCK}

\subsection{Introduction}

First, CheLongPu Fault of Taiwan Island (the causative fault of Chi-Chi earthquake) is introduced in this chapter and then other source parameters used in the simulation model are described. The geometrical parameters of the main shock (eg. epicentral location, focus depth, magnitude) from USGS, Harvard University and local authorities are compared to assess the most reasonable values. The shear wave velocity information of the upper 30 meters soil profile is used to classify the stations classes according to the NEHRP classification scheme (Borcherdt, 1995). Then the site amplification functions for both rock and soil stations that have such velocity information are calculated. The high frequency diminution operator, as introduced by Anderson and Hough (1984) is then analyzed.

In the stochastic simulation, the attenuation function is an important factor controlling the shape and amplitudes of seismograms at the recording station. Regression analysis of the main shock data is performed using the PGA data of the rock stations to approximate the geometrical spreading coefficients and a close-in distance saturation term, $\Delta$. Then the quality factor of the crust (Q) attached to this attenuation model is derived by regression analysis of the Fourier acceleration amplitude. The source duration of the motion, which is related to the rise time of the main shock, is calculated. Since it affects the duration time of the simulated seismogram at the 
recording stations, the duration model versus distance is derived using the defined shear wave window for each record, as discussed in Chapter 3.

\subsection{Geometric Parameters of the Main Shock}

\subsubsection{Fault Plane}

CheLongPu Fault is a major pre-existing fault that ruptured during the 1999 Chi-Chi earthquake. There are several models concerning the fault plane and its slip distribution (Wang et al., 2001; Sokolov et al., 2002; Irikura et al., 2000; Kikuchi et al., 2000). In our simulations, both a random slip distribution model and a predetermined slip distribution model are considered in order to compare the effects of the different fault rupture processes on the simulation. According to publications about CheLongPu Fault (Wang et al., 2001; Sokolov et al., 2002; Irikura et al., 2000), its strike and dip are $5^{\circ}$ and $30^{\circ}$, respectively. The fault plane and the fault grid are shown in Figure 4.1.

The pre-determined fault slip model used in the simulation is shown in Figure 2. (Wang et al., 2001). The slip contour is re-plotted in Figure 4.2 according to the model provided by the author.

\subsubsection{Main Shock Location}

According to USGS, the epicenter of the main shock was at $23.77 \mathrm{~N} 120.98 \mathrm{E}$ with moment magnitude 7.6 . It was reported as $\left(24.15 \mathrm{~N} 120.80 \mathrm{E}\right.$ with $M_{W}=7.6$ at focal depth $=21.2 \mathrm{~km})$ and $\left(23.85 \mathrm{~N} 120.82 \mathrm{E}, M_{W}=7.6\right.$, focal depth=8.0km) by Harvard CM'T solution and CWB, respectively. I choose the parameters from CWB for use in this study, as they incorporate more local data. 


\subsection{Site Classification}

All of the used 411 stations are classified as rock/soft-rock stations (NEHRP class B \& C) or soil stations (NEHRP class D \& E) according to the upper 30 meters shear wave velocity information provided by PEER (2005). NEHRP site classification schemes are listed in Table 4.1. The shear wave velocity information was gathered either by near surface geophysical surveys or by borehole geophysical investigations. However, not all of the stations have this information. For the stations for which no geophysical investigations were performed, researchers subjectively assigned the shear wave velocity according to its surface geology condition of each station (PEER, 2005). For stations with no accurate velocity information, the site classification used by Lee et al. (2001a) and by Phung et al. (2004) are used as a reference. Assigned NEHRP classes for each station are given in Table 3.1 . 
Table 4.1: NEHRP site classification scheme (Borcherdt, 1995)

\begin{tabular}{|l|c|c|}
\hline Site Class & $\overline{V_{s}}$ & Description \\
\hline A & $>1500 \mathrm{~m} / \mathrm{s}$ & Hard rock \\
\hline B & 760 to $1500 \mathrm{~m} / \mathrm{s}$ & Rock \\
\hline C & 360 to $760 \mathrm{~m} / \mathrm{s}$ & Very dense soil and soft rock \\
\hline D & 180 to $360 \mathrm{~m} / \mathrm{s}$ & Stiff soil \\
\hline E & $<180 \mathrm{~m} / \mathrm{s}$ & Soil \\
\hline
\end{tabular}

For the study database, 4 stations are NEHRP class B; 219 stations are NEHRP class C; 179 stations are NEHRP class D and 9 stations are NEHRP class E sites.

The soft rock stations (NEHRP class C) are used to calibrate the input source model for stochastic simulation (determine the two free parameters: stress drop and pulsing percentage). Then the soil stations are used with the calibrated input model to study the non-linear soil response.

\subsection{Kappa Factor}

The kappa factor introduced by Anderson and Hough (1984) is used to describe the high frequency diminution filter, defined as $D(f)=A_{0} \exp (-\pi \kappa f), f>f_{o}$ (Equation 2.9), where $f_{0}$ is the corner frequency. For all of the stations used, the kappa value is calculated separately for two horizontal components and the vertical component.

In this study, the frequency span to calculate kappa is defined visually for each 
record as shown in Figure 4.3. Generally, the corner frequency, $f_{0}$, is always considered to be well below $1 \mathrm{~Hz}$ for large earthquakes such as Chi-Chi earthquake and the starting frequency is visually defined at least beyond $1 \mathrm{~Hz}$. The ending frequency to calculate kappa is also visually defined, but not beyond $20 \mathrm{~Hz}$ where the noise dominates the signal in most cases. The $\kappa$ is calculated using the smoothed Fourier acceleration amplitude within the chosen frequency span by linear least square regression method. We have:

$$
\log [a(f)]=\log \left(A_{0}\right)-\log (e) \pi k f, \quad f>f_{0}
$$

Re-writing Equation 4.1 as $Y(x)=b+a x$, where $f$ is substituted by $x, Y(x)$ is $\log [a(f)]$ and $b$ is the constant related with source, the slope $a$ is $\pi \kappa \log (e)$.

Thus the kappa value can be derived as:

$$
\kappa=a /(\pi \log (e))
$$

$\kappa$ depends on distance $\left(\kappa=\kappa_{0}-C R\right)$ since anelastic attenuation effects are included, the value $k_{0}$ (pronounced kappa not), which denotes the zero-distance intercept of kappa, needs to be derived and input to simulations. After calculating the kappa value for all of the stations, the $k_{0}$ value can be regressed using least squares method. I regress the $k$ of vertical component, E-W component and N-S component for both rock stations and soil stations. Figure 4.4, Figure 4.5 and Figure 4.6 are the $k$ for rock stations on vertical component, N-S and E-W components while Figure 4.7, Figure 4.8 and Figure 4.9 are for soil stations on vertical component, N-S and E-W components.

According to the regression results, $\kappa_{0}$ is almost the same for the horizontal 
components of both rock $\left(\kappa_{0}=0.064\right)$ and soil $\left(\kappa_{0}=0.066\right)$ stations in Taiwan. However, $\kappa_{0}$ for vertical components are above 0.05 for both rock and soil stations.

\subsection{Wave Propagation Analysis}

\subsubsection{Attenuation Regression}

The ground motion attenuation relationship plays an important role in the stochastic modeling since it shows how the seismic energy decays with distance. The maximum distance to the fault for stations in the dataset is about $160 \mathrm{~km}$ and there are only about 60 of 400 stations with rupture-station distances greater than 100 $\mathrm{km}$. The paucity of far field records results in the inapplicability of detailed trilinear or bilinear models which are typical of attenuation over large distances (eg. Atkinson, 2004). We choose a simple function, as given in Equation 4.3, to define the attenuation model:

$$
\log (F A)=c_{1}+c_{2} \cdot \log (R)+c_{3} \cdot(R)
$$

where: $F A$ is the Fourier acceleration amplitude, PGA or PGV depending on the application; $R$ is the distance metric (see below); $c_{2}$ is the geometric spreading coefficient; and $c_{3}$ is anelastic attenuation coefficient.

A special feature of the Chi-Chi strong motion database is its richness of the near-source records. The shortest rupture-station distance in the dataset is less than $1 \mathrm{~km}$ and more than 30 stations have rupture-station distance less than $10 \mathrm{~km}$. Since near-source records attenuate slowly with distance due to the effect of the fault plane, I introduced a close-in distance saturation coefficient (denoted " $\Delta$ ") to control the shape of the attenuation curve in the near source. Thus the distance metric in Equation 4.3 is: $R=$ Dist $+\Delta$, where $\Delta$ is the saturation variable (in $\mathrm{km}$ ). 
According to the assumption that the site amplification for the vertical component is negligible at the rock stations, I first regress the PGA of vertical component for the rock stations to roughly determine the geometric spreading coefficient $c_{2}$ and $\Delta$ for Equation 4.3. The reason for this step is that in the regression process for Fourier acceleration amplitude, these two coefficients will be used to derived the anelastic coefficient of Equation 4.3 and then to calculate the Q model. In the regression, the geometric spreading coefficient is varied from -0.5 to -1.0 with step 0.1 and the $\Delta$ is varied from 1 to 20 in order to find the coefficients which provide the minimum root mean square (rms) of residuals, where the $\mathrm{rms}$ is defined as $\sqrt{\frac{\sum_{j=1}^{n}\left(\left(F A_{o b s}\right)_{j}-\left(F A_{r e g}\right)_{j}\right)^{2}}{n}}$, where $n$ is the number of the stations used for regression, $\left(F A_{o b s}\right)_{j}$ is the Fourier spectrum of the recorded data and $\left(F A_{r e g}\right)_{j}$ is the Fourier spectrum of the regressed result. The model with minimum rms of residuals has geometrical spreading coefficient -0.9 and $\Delta=7 \mathrm{~km}$. Figure 4.10 (a) is the regressed result of the attenuation curve for PGA, along with the rock station database. Figure 4.10 (b) is the residual distribution of the regressed result; there is no trend of the residuals, indicating the reasonableness of the chosen attenuation model.

The regressed geometric spreading coefficients are very close to the recent published attenuation relation (Liu and Tsai, 2005) for Taiwan region using a larger small-intermediate magnitude earthquake database. This is very important for the extended fault stochastic simulation method because the moment magnitude of the subfaults are usually low (In most case, it is about moment magnitude 4-5). By contrast, the attenuation model regressed above uses the dataset of a high magnitude earthquake $\left(M_{W}=7.6\right)$, but it may not be suitable for characterizing subfaults or low magnitude earthquakes. The geometric attenuation model is used as a constraint condition for the regression of Fourier acceleration amplitudes to determine Q. Fig- 
ure 4.11 (a) and Figure 4.11 (b) show the regressed curve and residual distribution for Fourier acceleration amplitude at $1 \mathrm{~Hz}$. Figure 4.12 (a) and Figure 4.12 (b) demonstrate the regressed result at $5 \mathrm{~Hz}$. The fit of the regressed curves and lack of trend of the residual distribution indicates that the geometric spreading coefficient and chosen $\Delta$ are acceptable.

\subsubsection{Q model}

The quality factor of crust (Q) is inversely related to the anelastic coefficient $c_{3}$ of Equation 4.3. The Q model can be represented as:

$$
Q=\frac{\pi \log (e) f}{c_{3} \beta}
$$

where $Q$ represents the shear wave $\mathrm{Q}$ model of the crust, $e$ is the base of natural log-

arithm, $f$ is the frequency, $\beta$ is shear wave velocity of the crust and $c_{3}$ is the anelastic coefficient of Equation 4.3. $c_{3}$ is a function of frequency and can be empirically determined by least squares. Figure 4.13 is the calculated $\mathrm{Q}$ model corresponding to the fixed geometric attenuation model (two exceptional points between $3 \mathrm{~Hz}$ and 5 $\mathrm{Hz}$ are removed when calculate $\mathrm{Q}$ model). The vertical bars are the $95 \%$ confidence interval.

\subsection{Site Amplification}

Site amplification is calculated considered two methods: the $\mathrm{H} / \mathrm{V}$ ratio and quarter wavelength approximation approaches. 


\subsubsection{H/V Ratio}

The H/V ratio approach was first proposed by Nakamura (1989) for application to micro-tremor measurements, based on the hypothesis that the amplification effects on the vertical component are small enough to be neglected while the observed amplification effects are developed on the horizontal component of ground motions. Lermo and Chavez-Garcia (1994) verified the applicability of the H/V ratio approach to shear waves from earthquakes by using strong motion earthquake data from Mexico earthquakes. Some theoretical justification of the $\mathrm{H} / \mathrm{V}$ ratio was provided by Lachet and Bard (1994).

According to Nakamura (1989), the site amplification function $(A(f))$ at one station is the ratio of the horizontal Fourier acceleration amplitude $\left(F A_{H}\right)$ to the vertical Fourier acceleration amplitude $\left(F A_{V}\right)$ defined as:

$$
A(f)=F A_{H} / F A_{V}
$$

\subsubsection{Quarter Wavelength Approximation Approach}

The specific site amplification function may be calculated for every station for which the shear wave velocity profile is known. There are 192 stations with such velocity profile provided by National Center for Research on Earthquake Engineering (NCREE) of Taiwan. The station's time-averaged shear wave velocity of the top 30 meter is calculated, and each station is classified into four categories according to the NEHRP site classification scheme of Borcherdt (1995). Among the used database , 5 station are NEHRP class B sites (list in Table 4.2); 65 stations are NEHRP class C sites (list in Table 4.3); 106 stations are NEHRP class D sites (list in Table 4.4) and 16 stations are NEHRP class E sites (list in Table 4.5). 
The site amplification is calculated from the seismic impedance using the quarterwavelength approach which was introduced by Boore and Joyner (1997). For a particular frequency, the amplification is given by the square root of the ratio between the seismic impedance averaged over a depth corresponding to a quarter wavelength and the seismic impedance at the depth of the source (Boore and Joyner, 1997). The algorithm to compute the site amplification is based on Equation 4.6:

$$
A[f(z)]=\sqrt{\rho_{s} \beta_{s} / \bar{\rho}(z) \bar{\beta}(z)}
$$

where $\rho_{s}$ and $\beta_{s}$ are the density and the shear wave velocity values in the vicinity of the source, $\bar{\beta}(z)$ is the average velocity to the depth $z$ which can be calculated by $z / S_{t t}(z)$ in which $S_{t t}(z)$ is the S travel time from the surface to depth $z, f(z)$ is the frequency function corresponding to the depth (can be obtained by $1 /\left[4 \mathrm{x} S_{t t}(z)\right]$ ), and $\bar{\rho}(z)$ is the travel-time-weighted average density (Boore and Joyner, 1997).

Table 4.2: Stations used to calculate site amplification based on the quarter wavelength approximation method (NEHRP class B sites)

\begin{tabular}{|l|c|c|c|c|}
\hline Station & $V_{s 30}(m / s)$ & Class & Amp $(1 \mathrm{~Hz})$ & $\operatorname{Amp}(5 \mathrm{~Hz})$ \\
\hline \hline KAU003 & 884.7 & B & 1.57 & 1.98 \\
\hline TAP065 & 1022.0 & B & 1.51 & 1.66 \\
\hline TAP071 & 825.2 & B & 1.56 & 1.92 \\
\hline TAP077 & 976.4 & B & 1.53 & 1.76 \\
\hline TAP114 & 1230.4 & B & 1.49 & 1.60 \\
\hline \hline
\end{tabular}


Table 4.3: Stations used to calculate site amplification based on the quarter wavelength approximation method (NEHRP class $\mathrm{C}$ sites)

\begin{tabular}{|l|c|c|c|c|}
\hline Station & $V_{s 30}(m / s)$ & Class & Amp $(1 \mathrm{~Hz})$ & Amp $(5 \mathrm{~Hz})$ \\
\hline \hline CHY006 & 422.5 & $\mathrm{C}$ & 1.67 & 2.55 \\
\hline CHY024 & 403.2 & $\mathrm{C}$ & 1.73 & 2.80 \\
\hline CHY028 & 539.1 & $\mathrm{C}$ & 1.05 & 1.40 \\
\hline CHY029 & 537.6 & $\mathrm{C}$ & 1.65 & 2.38 \\
\hline CHY034 & 374.8 & $\mathrm{C}$ & 1.87 & 3.29 \\
\hline CHY038 & 360.2 & $\mathrm{C}$ & 1.85 & 3.27 \\
\hline CHY041 & 480.9 & $\mathrm{C}$ & 1.73 & 2.78 \\
\hline CHY074 & 514.5 & $\mathrm{C}$ & 1.66 & 2.51 \\
\hline HWA026 & 450.8 & $\mathrm{C}$ & 1.73 & 2.88 \\
\hline HWA033 & 380.4 & $\mathrm{C}$ & 1.80 & 3.39 \\
\hline HWA034 & 373.3 & $\mathrm{C}$ & 1.81 & 3.17 \\
\hline HWA056 & 514.2 & $\mathrm{C}$ & 1.67 & 2.48 \\
\hline ILA020 & 454.5 & $\mathrm{C}$ & 1.68 & 2.51 \\
\hline ILA031 & 641.0 & $\mathrm{C}$ & 1.60 & 2.20 \\
\hline ILA046 & 383.3 & $\mathrm{C}$ & 1.85 & 3.25 \\
\hline KAU039 & 461.2 & $\mathrm{C}$ & 1.70 & 2.65 \\
\hline TAP035 & 392.2 & $\mathrm{C}$ & 1.78 & 3.42 \\
\hline TAP051 & 393.0 & $\mathrm{C}$ & 1.81 & 3.32 \\
\hline TAP056 & 604.9 & $\mathrm{C}$ & 1.58 & 2.04 \\
\hline TAP066 & 642.1 & $\mathrm{C}$ & 1.58 & 2.02 \\
\hline TAP089 & 395.6 & $\mathrm{C}$ & 1.71 & 4.37 \\
\hline TAP103 & 433.2 & $\mathrm{C}$ & 1.69 & 2.81 \\
\hline TCU003 & 504.0 & $\mathrm{C}$ & 1.63 & 2.33 \\
\hline TCU005 & 501.5 & $\mathrm{C}$ & 1.65 & 2.45 \\
\hline TCU009 & 463.7 & $\mathrm{C}$ & 1.72 & 2.68 \\
\hline TCU017 & 563.1 & $\mathrm{C}$ & 1.65 & 2.42 \\
\hline TCU019 & 499.4 & $\mathrm{C}$ & 1.70 & 2.61 \\
\hline TCU021 & 445.5 & $\mathrm{C}$ & 1.67 & 2.53 \\
\hline TCU027 & 661.4 & $\mathrm{C}$ & 1.62 & 2.23 \\
\hline TCU028 & 514.7 & $\mathrm{C}$ & 1.68 & 2.61 \\
\hline TCU030 & 615.1 & $\mathrm{C}$ & 1.61 & 2.17 \\
\hline TCU033 & 408.5 & $\mathrm{C}$ & 1.77 & 3.14 \\
\hline \hline
\end{tabular}

Continued... 


\begin{tabular}{|l|c|c|c|c|}
\hline Station & $V_{s 30}(\mathrm{~m} / \mathrm{s})$ & $\mathrm{Class}$ & $\mathrm{Amp}(1 \mathrm{~Hz})$ & $\mathrm{Amp}(5 \mathrm{~Hz})$ \\
\hline \hline TCU037 & 452.6 & $\mathrm{C}$ & 1.73 & 2.96 \\
\hline TCU039 & 543.7 & $\mathrm{C}$ & 1.69 & 2.54 \\
\hline TCU045 & 683.6 & $\mathrm{C}$ & 1.56 & 1.96 \\
\hline TCU046 & 442.7 & $\mathrm{C}$ & 1.69 & 3.26 \\
\hline TCU047 & 510.8 & $\mathrm{C}$ & 1.71 & 2.75 \\
\hline TCU049 & 466.3 & $\mathrm{C}$ & 1.70 & 2.66 \\
\hline TCU052 & 575.3 & $\mathrm{C}$ & 1.66 & 2.48 \\
\hline TCU053 & 449.3 & $\mathrm{C}$ & 1.74 & 2.74 \\
\hline TCU054 & 429.7 & $\mathrm{C}$ & 1.73 & 2.80 \\
\hline TCU067 & 437.9 & $\mathrm{C}$ & 1.77 & 2.92 \\
\hline TCU068 & 480.3 & $\mathrm{C}$ & 1.72 & 2.87 \\
\hline TCU070 & 392.9 & $\mathrm{C}$ & 1.80 & 3.13 \\
\hline TCU071 & 597.0 & $\mathrm{C}$ & 1.61 & 2.30 \\
\hline TCU072 & 459.6 & $\mathrm{C}$ & 1.66 & 2.68 \\
\hline TCU074 & 549.9 & $\mathrm{C}$ & 1.68 & 2.50 \\
\hline TCU075 & 511.1 & $\mathrm{C}$ & 1.65 & 2.44 \\
\hline TCU076 & 562.4 & $\mathrm{C}$ & 1.63 & 2.29 \\
\hline TCU077 & 503.5 & $\mathrm{C}$ & 1.65 & 2.42 \\
\hline TCU078 & 438.0 & $\mathrm{C}$ & 1.72 & 2.81 \\
\hline TCU082 & 464.3 & $\mathrm{C}$ & 1.73 & 2.77 \\
\hline TCU090 & 535.3 & $\mathrm{C}$ & 1.64 & 2.51 \\
\hline TCU094 & 573.3 & $\mathrm{C}$ & 1.65 & 2.41 \\
\hline TCU095 & 443.5 & $\mathrm{C}$ & 1.77 & 2.99 \\
\hline TCU102 & 691.1 & $\mathrm{C}$ & 1.59 & 2.13 \\
\hline TCU103 & 487.6 & $\mathrm{C}$ & 1.71 & 2.67 \\
\hline TCU116 & 458.7 & $\mathrm{C}$ & 1.71 & 2.71 \\
\hline TCU120 & 460.0 & $\mathrm{C}$ & 1.03 & 1.20 \\
\hline TCU128 & 582.8 & $\mathrm{C}$ & 1.66 & 2.46 \\
\hline TCU138 & 600.0 & $\mathrm{C}$ & 1.63 & 2.26 \\
\hline TCU143 & 457.0 & $\mathrm{C}$ & 1.71 & 2.80 \\
\hline TCU147 & 523.8 & $\mathrm{C}$ & 1.63 & 2.35 \\
\hline TCU148 & 497.9 & $\mathrm{C}$ & 1.66 & 2.52 \\
\hline TTN041 & 420.8 & $\mathrm{C}$ & 1.76 & 2.95 \\
\hline \hline & & & & \\
\hline
\end{tabular}


Table 4.4: Stations used to calculate site amplification based on the quarter wavelength approximation method (NEHRP class D sites)

\begin{tabular}{|l|c|c|c|c|}
\hline Station & $V_{s 30}(m / s)$ & Class & Amp $(1 \mathrm{~Hz})$ & Amp $(5 \mathrm{~Hz})$ \\
\hline \hline CHY001 & 225.1 & $\mathrm{D}$ & 2.34 & 4.56 \\
\hline CHY002 & 228.1 & $\mathrm{D}$ & 2.21 & 3.90 \\
\hline CHY004 & 269.3 & $\mathrm{D}$ & 2.08 & 4.06 \\
\hline CHY005 & 208.1 & $\mathrm{D}$ & 2.45 & 4.17 \\
\hline CHY007 & 199.4 & $\mathrm{D}$ & 2.43 & 4.95 \\
\hline CHY008 & 207.4 & $\mathrm{D}$ & 2.32 & 4.37 \\
\hline CHY009 & 222.4 & $\mathrm{D}$ & 2.45 & 4.37 \\
\hline CHY012 & 189.9 & $\mathrm{D}$ & 2.43 & 5.18 \\
\hline CHY013 & 212.4 & $\mathrm{D}$ & 2.52 & 5.09 \\
\hline CHY015 & 221.6 & $\mathrm{D}$ & 2.62 & 4.72 \\
\hline CHY016 & 197.3 & $\mathrm{D}$ & 2.74 & 4.61 \\
\hline CHY017 & 188.1 & $\mathrm{D}$ & 2.37 & 4.94 \\
\hline CHY020 & 210.0 & $\mathrm{D}$ & 2.53 & 5.05 \\
\hline CHY021 & 209.8 & $\mathrm{D}$ & 2.47 & 4.35 \\
\hline CHY025 & 274.8 & $\mathrm{D}$ & 2.09 & 3.65 \\
\hline CHY026 & 218.3 & $\mathrm{D}$ & 2.41 & 4.70 \\
\hline CHY027 & 206.0 & $\mathrm{D}$ & 2.37 & 4.89 \\
\hline CHY030 & 205.4 & $\mathrm{D}$ & 2.32 & 4.64 \\
\hline CHY031 & 214.2 & $\mathrm{D}$ & 2.55 & 4.41 \\
\hline CHY032 & 190.1 & $\mathrm{D}$ & 2.59 & 4.76 \\
\hline CHY033 & 191.1 & $\mathrm{D}$ & 2.72 & 4.83 \\
\hline CHY036 & 206.1 & $\mathrm{D}$ & 2.31 & 4.59 \\
\hline CHY037 & 204.9 & $\mathrm{D}$ & 2.70 & 5.14 \\
\hline CHY039 & 187.4 & $\mathrm{D}$ & 2.81 & 4.72 \\
\hline CHY043 & 223.1 & $\mathrm{D}$ & 2.49 & 4.52 \\
\hline CHY044 & 190.5 & $\mathrm{D}$ & 2.87 & 4.61 \\
\hline CHY048 & 233.0 & $\mathrm{D}$ & 1.97 & 5.07 \\
\hline CHY049 & 221.9 & $\mathrm{D}$ & 2.57 & 4.66 \\
\hline CHY051 & 353.8 & $\mathrm{D}$ & 1.83 & 3.84 \\
\hline CHY055 & 218.5 & $\mathrm{D}$ & 2.39 & 4.44 \\
\hline CHY056 & 187.0 & $\mathrm{D}$ & 2.87 & 5.06 \\
\hline CHY059 & 185.2 & $\mathrm{D}$ & 2.61 & 4.86 \\
\hline \hline
\end{tabular}

Continued... 


\begin{tabular}{|l|c|c|c|c|}
\hline Station & $V_{s 30}(\mathrm{~m} / \mathrm{s})$ & Class & $\mathrm{Amp}(1 \mathrm{~Hz})$ & $\mathrm{Amp}(5 \mathrm{~Hz})$ \\
\hline \hline CHY060 & 213.2 & $\mathrm{D}$ & 2.18 & 4.40 \\
\hline CHY066 & 206.4 & $\mathrm{D}$ & 1.36 & 5.03 \\
\hline CHY067 & 224.8 & $\mathrm{D}$ & 2.35 & 4.81 \\
\hline CHY068 & 188.6 & $\mathrm{D}$ & 2.44 & 4.81 \\
\hline CHY073 & 191.0 & $\mathrm{D}$ & 2.02 & 4.57 \\
\hline CHY082 & 191.5 & $\mathrm{D}$ & 2.92 & 5.28 \\
\hline CHY084 & 257.8 & $\mathrm{D}$ & 2.15 & 4.00 \\
\hline CHY092 & 248.9 & $\mathrm{D}$ & 2.27 & 4.14 \\
\hline CHY093 & 189.1 & $\mathrm{D}$ & 2.24 & 4.99 \\
\hline CHY094 & 221.5 & $\mathrm{D}$ & 2.57 & 4.48 \\
\hline CHY095 & 212.3 & $\mathrm{D}$ & 2.55 & 4.88 \\
\hline CHY099 & 209.8 & $\mathrm{D}$ & 2.22 & 4.42 \\
\hline CHY101 & 255.0 & $\mathrm{D}$ & 2.09 & 3.96 \\
\hline CHY103 & 218.8 & $\mathrm{D}$ & 2.46 & 4.84 \\
\hline CHY104 & 219.9 & $\mathrm{D}$ & 2.04 & 3.77 \\
\hline CHY105 & 202.1 & $\mathrm{D}$ & 2.37 & 4.81 \\
\hline CHY106 & 224.5 & $\mathrm{D}$ & 2.42 & 4.52 \\
\hline CHY111 & 268.2 & $\mathrm{D}$ & 2.16 & 3.73 \\
\hline CHY112 & 237.3 & $\mathrm{D}$ & 2.45 & 4.35 \\
\hline CHY113 & 237.2 & $\mathrm{D}$ & 2.41 & 4.49 \\
\hline HWA004 & 312.6 & $\mathrm{D}$ & 1.99 & 4.16 \\
\hline ILA003 & 264.8 & $\mathrm{D}$ & 1.96 & 3.82 \\
\hline ILA005 & 236.3 & $\mathrm{D}$ & 2.03 & 4.05 \\
\hline ILA006 & 274.8 & $\mathrm{D}$ & 1.89 & 3.56 \\
\hline ILA013 & 192.5 & $\mathrm{D}$ & 1.93 & 3.90 \\
\hline ILA014 & 298.8 & $\mathrm{D}$ & 1.88 & 3.50 \\
\hline ILA016 & 268.8 & $\mathrm{D}$ & 1.96 & 3.63 \\
\hline ILA026 & 235.1 & $\mathrm{D}$ & 2.22 & 3.78 \\
\hline ILA027 & 213.9 & $\mathrm{D}$ & 1.99 & 4.12 \\
\hline ILA028 & 215.1 & $\mathrm{D}$ & 2.40 & 4.97 \\
\hline ILA029 & 185.1 & $\mathrm{D}$ & 2.55 & 5.02 \\
\hline ILA030 & 197.4 & $\mathrm{D}$ & 2.61 & 4.57 \\
\hline ILA032 & 296.9 & $\mathrm{D}$ & 1.85 & 3.37 \\
\hline ILA037 & 207.2 & $\mathrm{D}$ & 2.34 & 4.33 \\
\hline ILA038 & 243.1 & $\mathrm{D}$ & 1.94 & 3.81 \\
\hline ILA039 & 222.6 & $\mathrm{D}$ & 1.88 & 3.54 \\
\hline \hline
\end{tabular}

Continued... 


\begin{tabular}{|l|c|c|c|c|}
\hline Station & $V_{s 30}(\mathrm{~m} / \mathrm{s})$ & Class & $\mathrm{Amp}(1 \mathrm{~Hz})$ & $\mathrm{Amp}(5 \mathrm{~Hz})$ \\
\hline \hline ILA041 & 192.1 & $\mathrm{D}$ & 2.93 & 4.73 \\
\hline ILA042 & 206.8 & $\mathrm{D}$ & 2.41 & 4.89 \\
\hline ILA048 & 190.9 & $\mathrm{D}$ & 2.39 & 4.82 \\
\hline ILA049 & 185.8 & $\mathrm{D}$ & 1.99 & 4.24 \\
\hline ILA055 & 263.0 & $\mathrm{D}$ & 2.28 & 4.05 \\
\hline ILA056 & 219.7 & $\mathrm{D}$ & 2.50 & 4.28 \\
\hline ILA059 & 231.4 & $\mathrm{D}$ & 2.11 & 4.05 \\
\hline KAU006 & 214.9 & $\mathrm{D}$ & 2.60 & 4.87 \\
\hline KAU008 & 279.6 & $\mathrm{D}$ & 2.08 & 4.22 \\
\hline KAU013 & 257.3 & $\mathrm{D}$ & 2.12 & 4.35 \\
\hline KAU014 & 243.1 & $\mathrm{D}$ & 2.06 & 3.68 \\
\hline KAU017 & 247.9 & $\mathrm{D}$ & 2.31 & 4.14 \\
\hline KAU020 & 340.5 & $\mathrm{D}$ & 1.77 & 3.10 \\
\hline KAU029 & 352.4 & $\mathrm{D}$ & 1.68 & 2.55 \\
\hline KAU035 & 277.4 & $\mathrm{D}$ & 1.96 & 3.72 \\
\hline KAU037 & 277.7 & $\mathrm{D}$ & 1.78 & 3.07 \\
\hline KAU046 & 192.6 & $\mathrm{D}$ & 2.63 & 4.76 \\
\hline KAU085 & 253.9 & $\mathrm{D}$ & 2.05 & 3.90 \\
\hline KAU089 & 190.5 & $\mathrm{D}$ & 2.12 & 4.70 \\
\hline TAP002 & 358.3 & $\mathrm{D}$ & 1.79 & 4.20 \\
\hline TAP003 & 206.9 & $\mathrm{D}$ & 2.70 & 4.76 \\
\hline TAP009 & 180.1 & $\mathrm{D}$ & 2.13 & 4.61 \\
\hline TAP010 & 217.6 & $\mathrm{D}$ & 2.54 & 4.69 \\
\hline TAP019 & 238.4 & $\mathrm{D}$ & 2.16 & 5.01 \\
\hline TAP024 & 183.7 & $\mathrm{D}$ & 3.02 & 5.54 \\
\hline TAP057 & 351.9 & $\mathrm{D}$ & 1.79 & 4.25 \\
\hline TAP084 & 196.4 & $\mathrm{D}$ & 2.25 & 4.59 \\
\hline TAP090 & 325.8 & $\mathrm{D}$ & 1.86 & 3.24 \\
\hline TAP093 & 186.8 & $\mathrm{D}$ & 2.81 & 5.48 \\
\hline TCU040 & 331.1 & $\mathrm{D}$ & 1.82 & 2.96 \\
\hline TCU065 & 285.0 & $\mathrm{D}$ & 2.10 & 4.04 \\
\hline TCU079 & 340.7 & $\mathrm{D}$ & 1.75 & 3.49 \\
\hline TCU086 & 219.8 & $\mathrm{D}$ & 2.50 & 4.53 \\
\hline TCU110 & 197.7 & $\mathrm{D}$ & 2.65 & 4.97 \\
\hline TCU111 & 230.7 & $\mathrm{D}$ & 2.24 & 4.63 \\
\hline TCU113 & 226.2 & $\mathrm{D}$ & 2.44 & 4.86 \\
\hline \hline
\end{tabular}

Continued... 


\begin{tabular}{|c|c|c|c|c|}
\hline Station & $V_{s 30}(m / s)$ & Class & Amp $(1 \mathrm{~Hz})$ & Amp $(5 \mathrm{~Hz})$ \\
\hline \hline TCU115 & 181.5 & $\mathrm{D}$ & 2.82 & 5.10 \\
\hline TCU139 & 305.8 & $\mathrm{D}$ & 2.01 & 3.46 \\
\hline \hline
\end{tabular}

Table 4.5: Stations used to calculate site amplification based on the quarter wavelength approximation method (NEHRP class E sites)

\begin{tabular}{|l|c|c|c|c|}
\hline Station & $V_{s 30}(\mathrm{~m} / \mathrm{s})$ & Class & Amp $(1 \mathrm{~Hz})$ & Amp $(5 \mathrm{~Hz})$ \\
\hline \hline CHY003 & 162.5 & $\mathrm{E}$ & 3.02 & 5.68 \\
\hline CHY047 & 144.4 & $\mathrm{E}$ & 2.22 & 5.22 \\
\hline CHY054 & 167.3 & $\mathrm{E}$ & 2.88 & 5.39 \\
\hline CHY076 & 156.6 & $\mathrm{E}$ & 3.09 & 6.02 \\
\hline CHY077 & 117.0 & $\mathrm{E}$ & 3.68 & 6.04 \\
\hline CHY078 & 150.7 & $\mathrm{E}$ & 2.55 & 5.57 \\
\hline CHY107 & 161.2 & $\mathrm{E}$ & 2.69 & 5.25 \\
\hline HWA059 & 144.6 & $\mathrm{E}$ & 2.22 & 5.23 \\
\hline ILA004 & 116.1 & $\mathrm{E}$ & 5.31 & 7.02 \\
\hline ILA044 & 151.1 & $\mathrm{E}$ & 2.93 & 5.24 \\
\hline KAU011 & 135.6 & $\mathrm{E}$ & 3.25 & 5.10 \\
\hline KAU023 & 160.3 & $\mathrm{E}$ & 2.23 & 4.76 \\
\hline KAU045 & 142.6 & $\mathrm{E}$ & 3.84 & 5.24 \\
\hline KAU048 & 168.2 & $\mathrm{E}$ & 1.99 & 4.46 \\
\hline TAP001 & 155.3 & $\mathrm{E}$ & 3.31 & 5.40 \\
\hline TAP022 & 175.0 & $\mathrm{E}$ & 2.65 & 5.54 \\
\hline \hline
\end{tabular}

The detailed upper 30 meter shear wave velocity information (provided by NCREE) is stored as an EXCEL file. For those stations with known profiles, velocity information is typically known up to 30 meters. The quarter wavelength approximation method (Boore and Joyner, 1997) is used to calculate the amplification function. Because the quarter wavelength method needs the shear wave velocity information up to the depth of the earthquake source, the formula from Table 1 of Boore and Joyner 
(1997) is used to extrapolate from 30 meters to provide the deeper shear wave velocity structure. Three evenly spaced sample depths are chosen for every interval in Table 1 of Boore and Joyner (1997). That is, within the 30 meter to 190 meter interval, three depths are calculated and velocities are estimated using:

$$
3.542 \cdot z^{0.407}
$$

where $Z$ is depth in meter (Boore and Joyner, 1997).

The shear wave velocity of top 30 meters and the extrapolated velocity structure of one rock station is shown as an example in Figure 4.14, while one soil station's velocity structure is shown in Figure 4.15. The density profile is calculated using Equation 3 of Boore and Joyner $(1997)\left(\rho(z)=2.5+[\beta(z)-0.3] \times \frac{2.8-2.5}{3.5-0.3}\right)$, with the minor modification shown below:

$$
\rho(z)=2.0+[\beta(z)-0.3] \times \frac{2.3-2.0}{3.5-0.3}
$$

In the above modified equation, the lower and upper limit of the density were assigned as 2.0 and 2.3 respectively, according to the information provided by NCREE (Taiwan), which are compare lower to the California values of 2.5 and 2.8 in the publication of Boore and Joyner (1997).

The shear wave velocity information stored in the EXCEL file is extracted using a Java program (written by myself), which calls the site amplification calculation program by Boore and Joyner (1997) as a black-box within Java. To meet the requirement of the site amplification program, the input velocity files were designed using the following assumptions:

1. The shear wave velocity increases with depth 
2. Deeper layers with slower velocity will be ignored

A sample input file for the site amplification program is shown in Table 4.6.

The calculated site amplification function of all stations is averaged for rock stations and soil stations. The averaged result of the rock stations (NEHRP class C) and soil stations (NEHRP class D); the site amplification function based on $\mathrm{H} / \mathrm{V}$ ratio approach and the site amplification of California (Boore and Joyner, 1997) are compared in Figure 4.16 and Figure 4.17. In Figure 4.16, the site amplification function of NEHRP class $\mathrm{C}$ sites at Taiwan and California is almost identical at high frequency $(>3 \mathrm{~Hz})$ but slightly different at lower frequency $(<3 \mathrm{~Hz})$. The $\mathrm{H} / \mathrm{V}$ ratio estimate of site amplification is close to these two amplification functions at low frequency $(<3 \mathrm{~Hz})$, but shows a significant decrease at the higher frequency $(>3 \mathrm{~Hz})$ due to the effect of the high-frequency diminution operator $\left(\kappa_{0}\right)$. Figure 4.17 is the comparison of the site amplification for NEHRP class D sites which shows the similar characteristic as Figure 4.16; the disagreement between $\mathrm{H} / \mathrm{V}$ and the theoretical estimate is significant at all frequencies. There is a noticeable difference in the high frequency between the quarter wavelength estimates for Taiwan VS. California, probably because the approach adopted to calculate the site amplification for NEHRP class D sites in California is different from that used in this study. The site amplification function for NEHRP class D sites in California is based on the empirical analysis (ratio of response spectra between sites with $\bar{V}_{30}=620 \mathrm{~m} / \mathrm{sec}$ and desired sites) and it was corrected from the site amplification function for sites with $\bar{V}_{30}=620 \mathrm{~m} / \mathrm{sec}$. Meanwhile, there was an assumption to calculate the site amplification function for California NEHRP class D sites that the ratio of Fourier and response spectra are the same which is not strictly true especially for the high frequency (Boore and Joyner, 1997). 
Table 4.6. Sample shear wave velocity structure to calculate the site amplification function by quarter wavelength approximation approach

$\begin{array}{ccc}\text { Sample input file to site_amp at station: CHYO02 } \\ \text { Depth } & \text { SVel } & \begin{array}{c}\text { Dens } \\ 0\end{array} \\ 232.55 & 2.00 \\ 10.5 & 285.71 & 2.00 \\ 11.0 & 294.11 & 2.00 \\ 16.0 & 303.03 & 2.00 \\ 16.5 & 312.50 & 2.00 \\ 28.0 & 322.58 & 2.00 \\ 83.0 & 1286.2 & 2.09 \\ 136.5 & 1574.8 & 2.12 \\ 190.0 & 1801.7 & 2.14 \\ 1460.0 & 2700.9 & 2.23 \\ 2730.0 & 3059.1 & 2.26 \\ 4000.0 & 3300.7 & 2.28 \\ 5333.0 & 3380.1 & 2.29 \\ 6666.0 & 3445.6 & 2.29 \\ 7999.0 & 3500.1 & 2.30 \\ \text { SVel }=\text { Shear wave velocity } \\ \text { Dens }=\text { Density }\end{array}$


The site effects at a specific site are the product of the site amplification function and the high frequency diminution operator $\left(\kappa_{0}\right)$. Thus in Figure 4.16 and Figure 4.17, the quarter wavelength amplification need to be multiplied by the $\kappa_{0}$ to obtain the net amplification. However, for the $\mathrm{H} / \mathrm{V}$ ratio approach, the kappa effect may have been removed by spectral division if the kappa value remains the same for the horizontal component and the vertical component.

In fact, the kappa operator value for the horizontal component is not equal to that of the vertical component (at least for Chi-Chi case). If $\kappa_{0 H}$ is equal to $\kappa_{0 V}$, $\frac{F A_{H}}{F A_{V}}$ should be used in the simulations as net site amplification. Thus, Equation 4.5 can be re-written as:

$$
\frac{F A_{H}}{F A_{V}}=\operatorname{Amp} \frac{\exp \left(-\pi f \kappa_{0 H}\right)}{\exp \left(-\pi f \kappa_{0 V}\right)}
$$

where Amp is the site amplification, $\kappa_{0 H}$ is kappa for horizontal component and $\kappa_{0 V}$ is kappa for vertical component. As mentioned in Chap 2, the site term in the stochastic simulations is referred as the product of the site amplification and the horizontal high frequency diminution operator.

Thus, $A(f)_{H / V} \cdot \exp \left(-\pi \kappa_{0 V}\right)$ which is the site term should be used in simulations when the $\kappa_{0}$ for vertical component is not considered as zero. Figure 4.18 and Figure 4.19 are the site term as the product of the site amplification and the high frequency diminution operator of the horizontal component (quarter wavelength approximation approach) and the product of the $\mathrm{H} / \mathrm{V}$ ratio and the vertical component high frequency diminution operator. According to Figure 4.18 and Figure 4.19, the site terms calculated from $\mathrm{H} / \mathrm{V}$ ratio method and the quarter wavelength approximation method are very similar within the frequency range of interest. Our results indicate that the $\mathrm{H} / \mathrm{V}$ ratio can be a good choice for the site amplification function if no shear wave velocity information is available. In Figure 4.18 and Figure 4.19, 
the site term at high frequency for Taiwan and California is different because of the different kappa value used for these two regions $\left(\kappa_{0 H}=0.07\right.$ in Taiwan and $\kappa_{0 H}=0.035$ in California).

\subsection{Duration of Motions}

In this study, the duration time of the seismogram is defined as follows. The start time of the signal is the arrival time of the shear wave which is picked visually from the seismogram; the end time of the signal is first picked visually but then compared with the calculated time to accumulate $90 \%$ of the energy of the whole seismogram. If the calculated time is earlier than the observed end time, then the calculated time is used as the end time of the signal. Duration is often expressed as a function of distance(R) as: (Atkinson and Boore, 1995)

$$
T(R)=T_{0}+d R
$$

$T_{0}$ is the source duration time which can be calculated using Equation 4.11

$$
T_{0}=\frac{1}{2 f_{0}}
$$

Equation 4.12 is used to obtain the corner frequency $f_{0}$ which was based on source parameters of earthquake records in ENA (Eastern North America) (Atkinson and Boore, 1995).

$$
\log \left(f_{0}\right)=2.41-0.533 M
$$

where $M$ is the moment magnitude of the target earthquake.

Figure 4.20 plots duration versus rupture-station distance. $T_{0}$ can be roughly 
determined as $26 \mathrm{sec}$ from the $\mathrm{Y}$-axis intercept value of Figure 4.20. The slope of the duration curve $d$ is 0.1 in this case. The $T_{0}$ value calculated from Equation 4.11 is about $22 \mathrm{sec}$, which is very close to the result inferred from observation. The agreement between the calculated $T_{0}$ and the observed one suggest that the definition of the shear wave window is reasonable in the study. With help of Equation 4.10, the duration time at any site can be determined. 


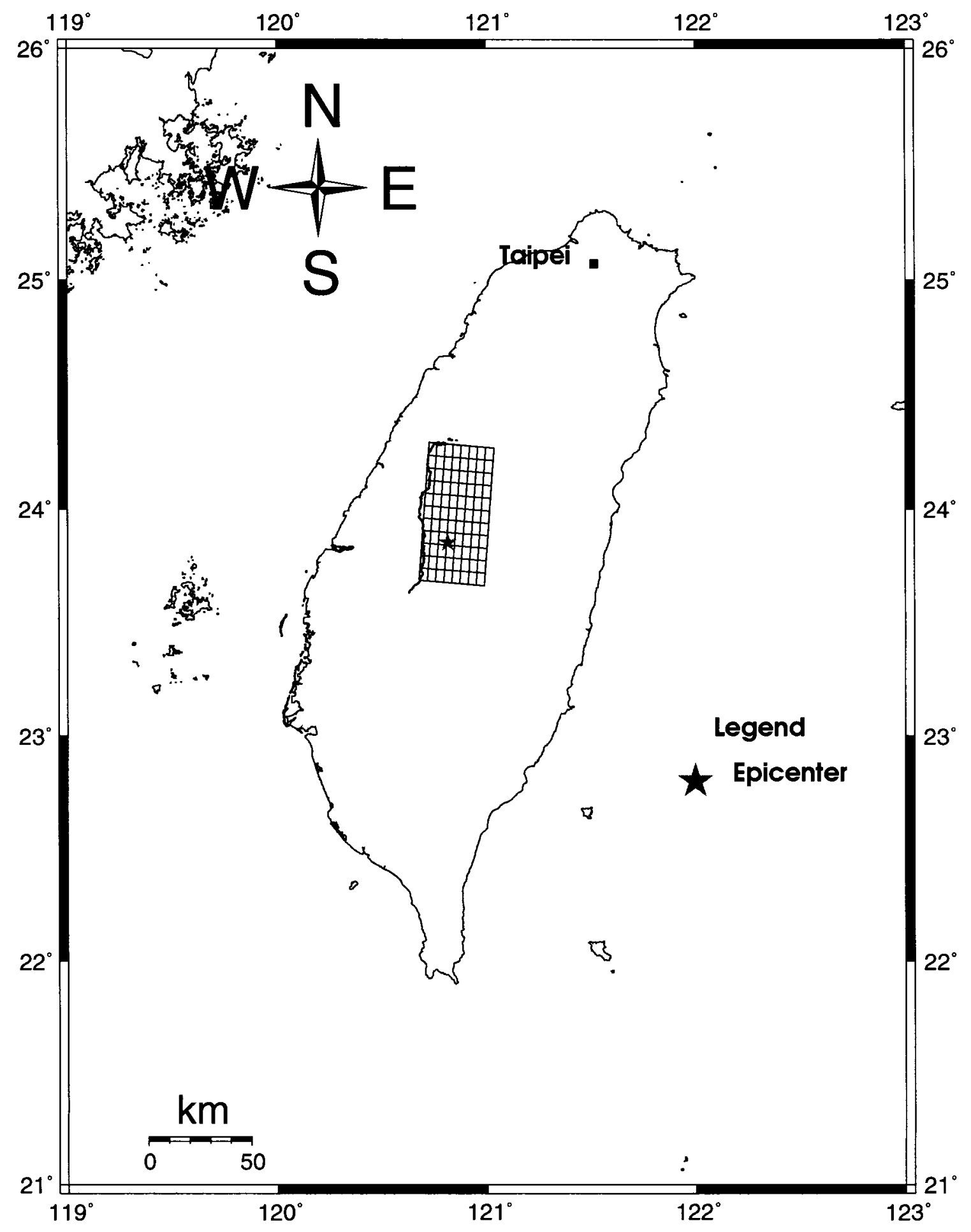

GMT 2005 Dec 01 02:38:01

Figure 4.1. The fault plane adopted (Wang et al., 2001) in this study 


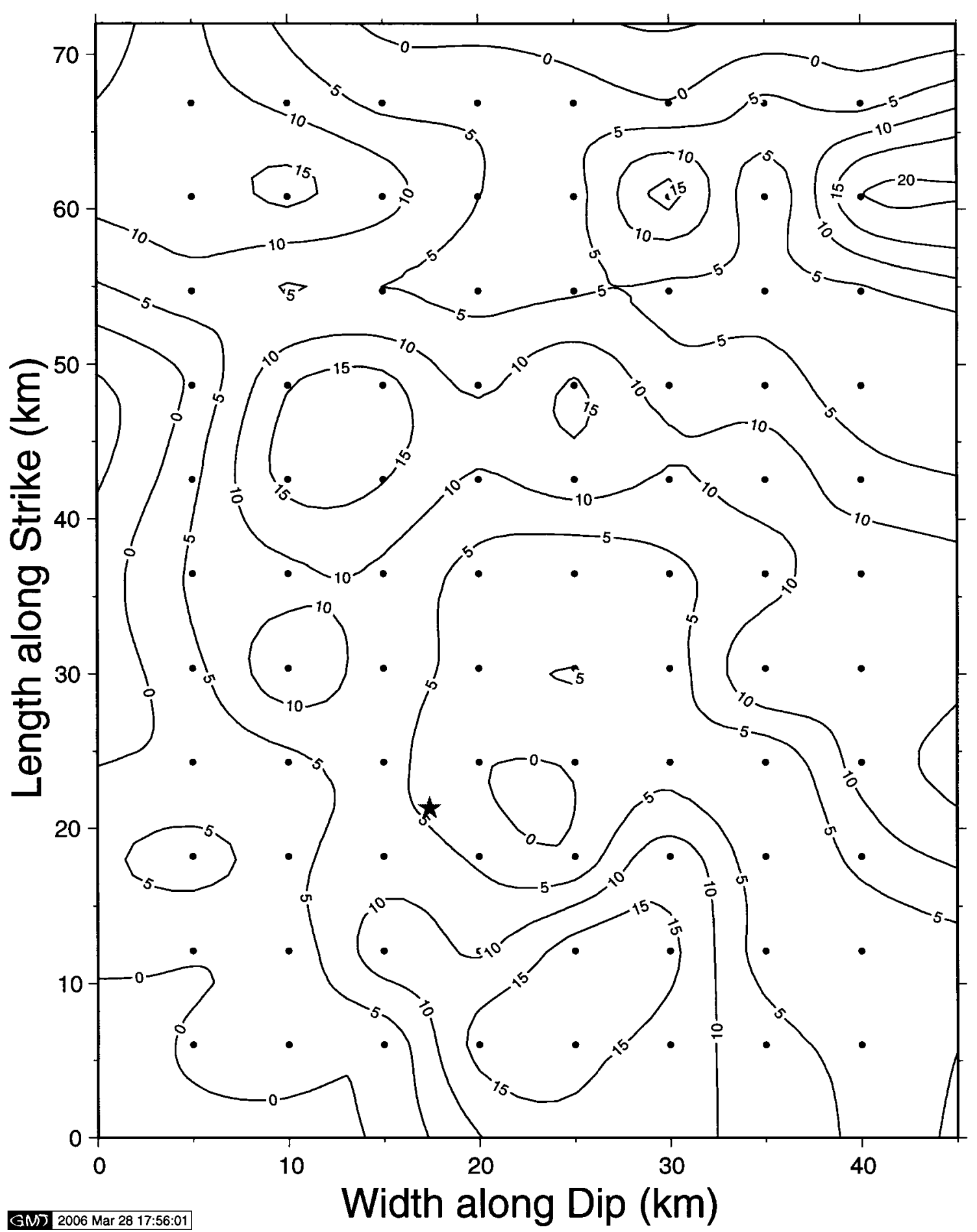

Figure 4.2. The pre-determined slip distribution model used in this study (Wang et al., 2001), the star is the epicenter; the downdip direction is along the bottom line $(0-40 \mathrm{~km})$ of the figure and the strike is along the left boundary $(0-70 \mathrm{~km})$ of the figure. 

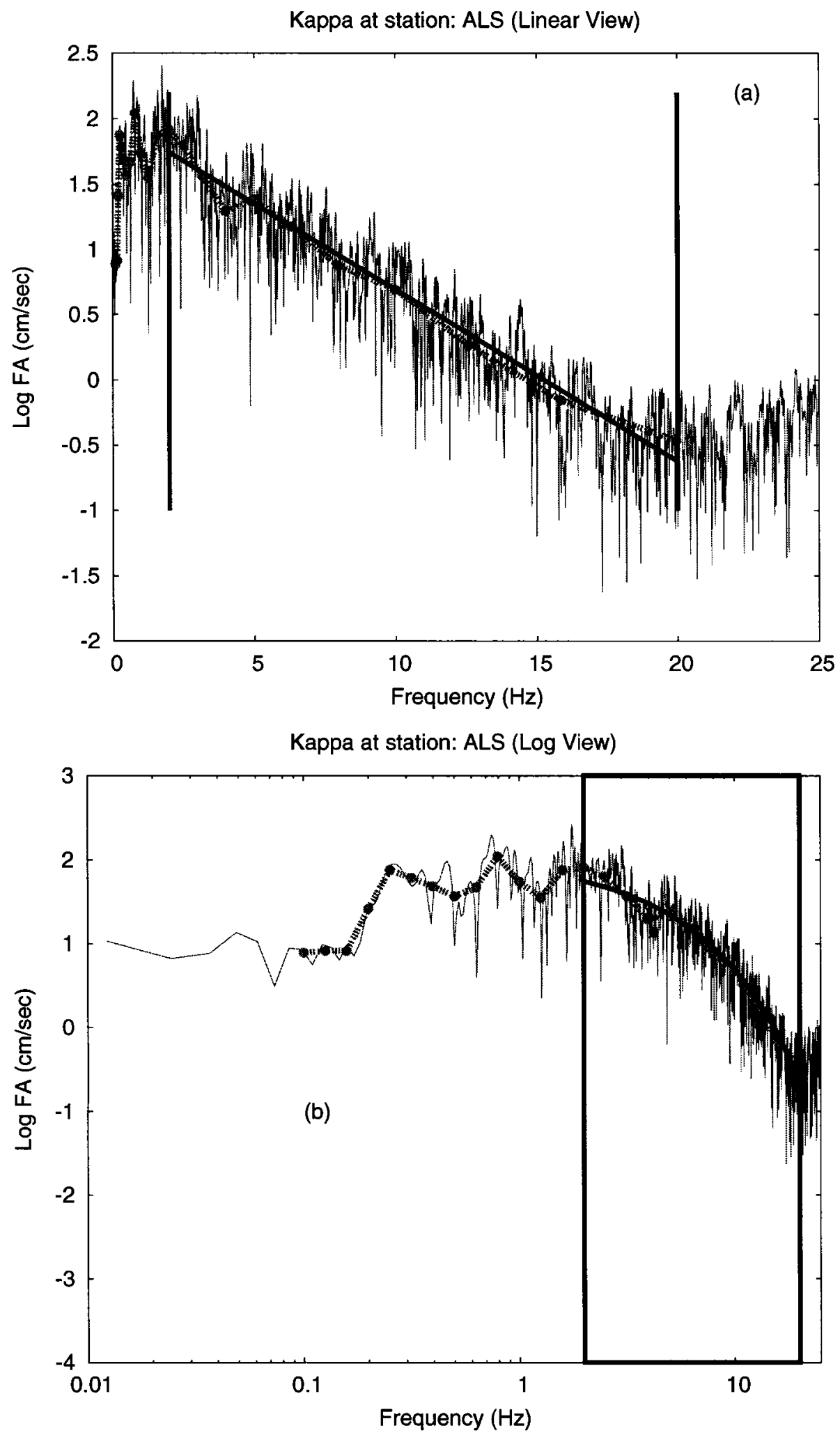

Figure 4.3. Sample Fourier acceleration amplitude to calculate $\kappa$ at station ALS: (a) Linear View (b) Log View 


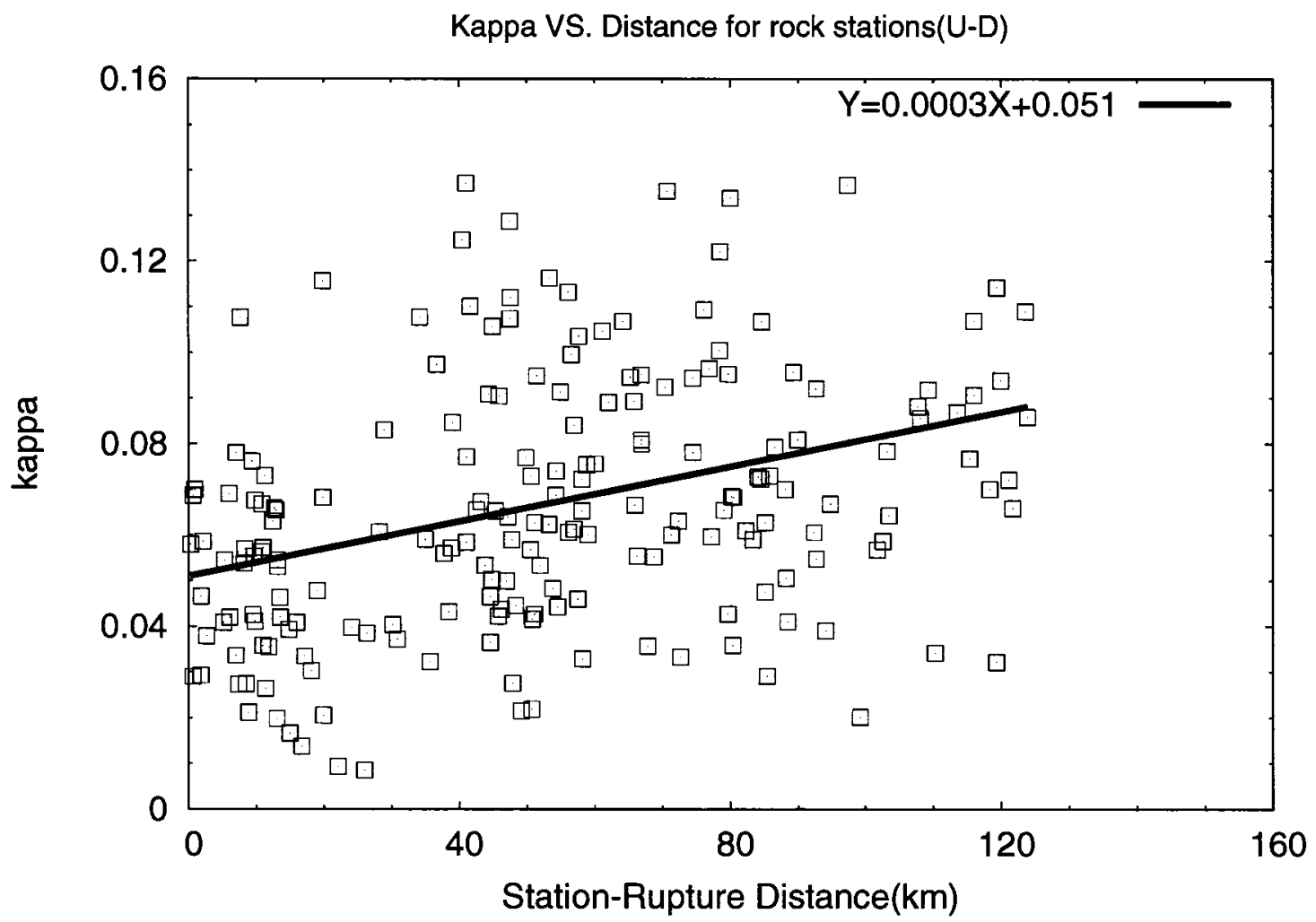

Figure 4.4. $\kappa$ versus station-rupture distance to calculate $\kappa_{0}$ of rock stations (U-D) 


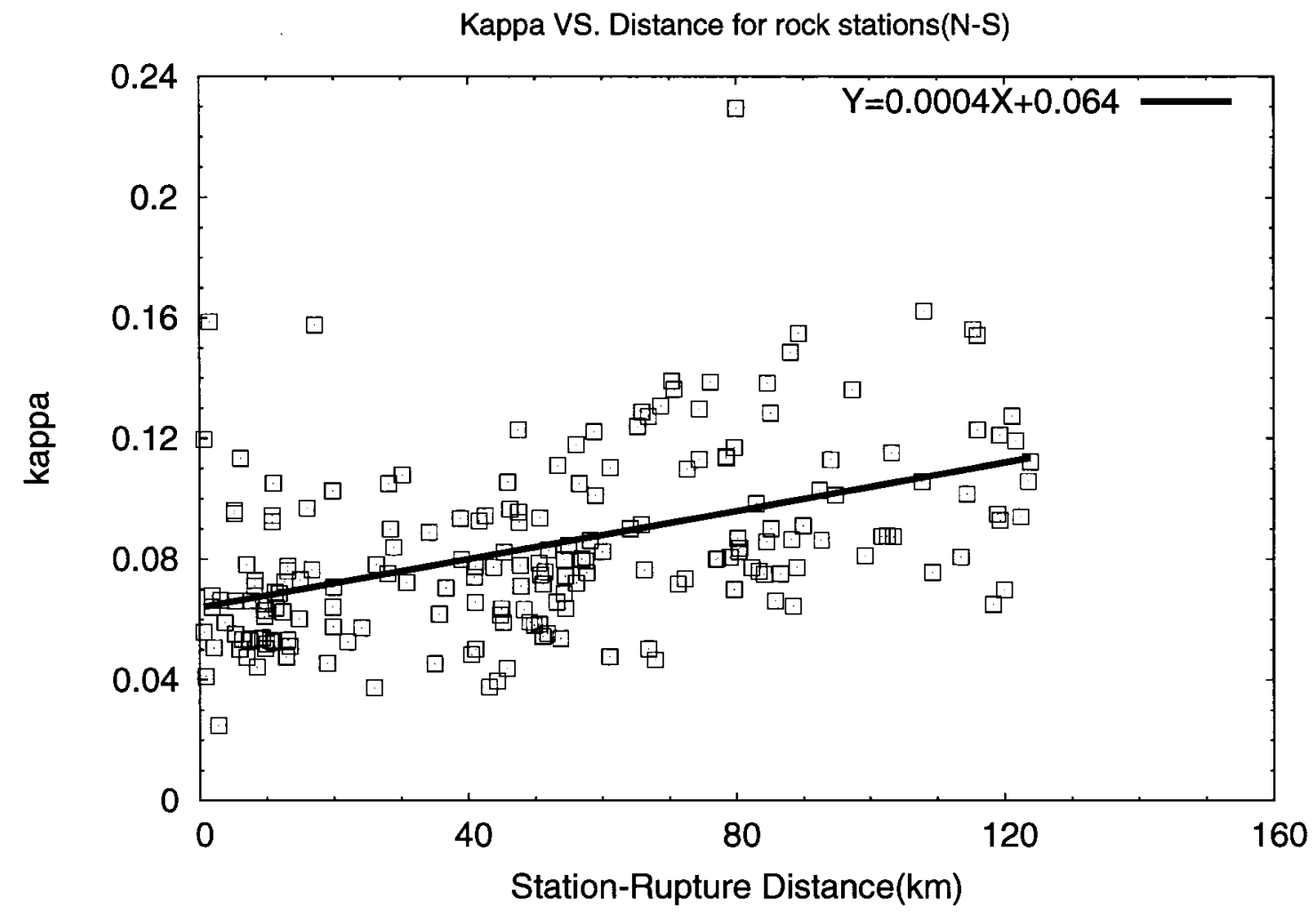

Figure 4.5. $\kappa$ versus station-rupture distance to calculate $\kappa_{0}$ of rock stations (N-S) 


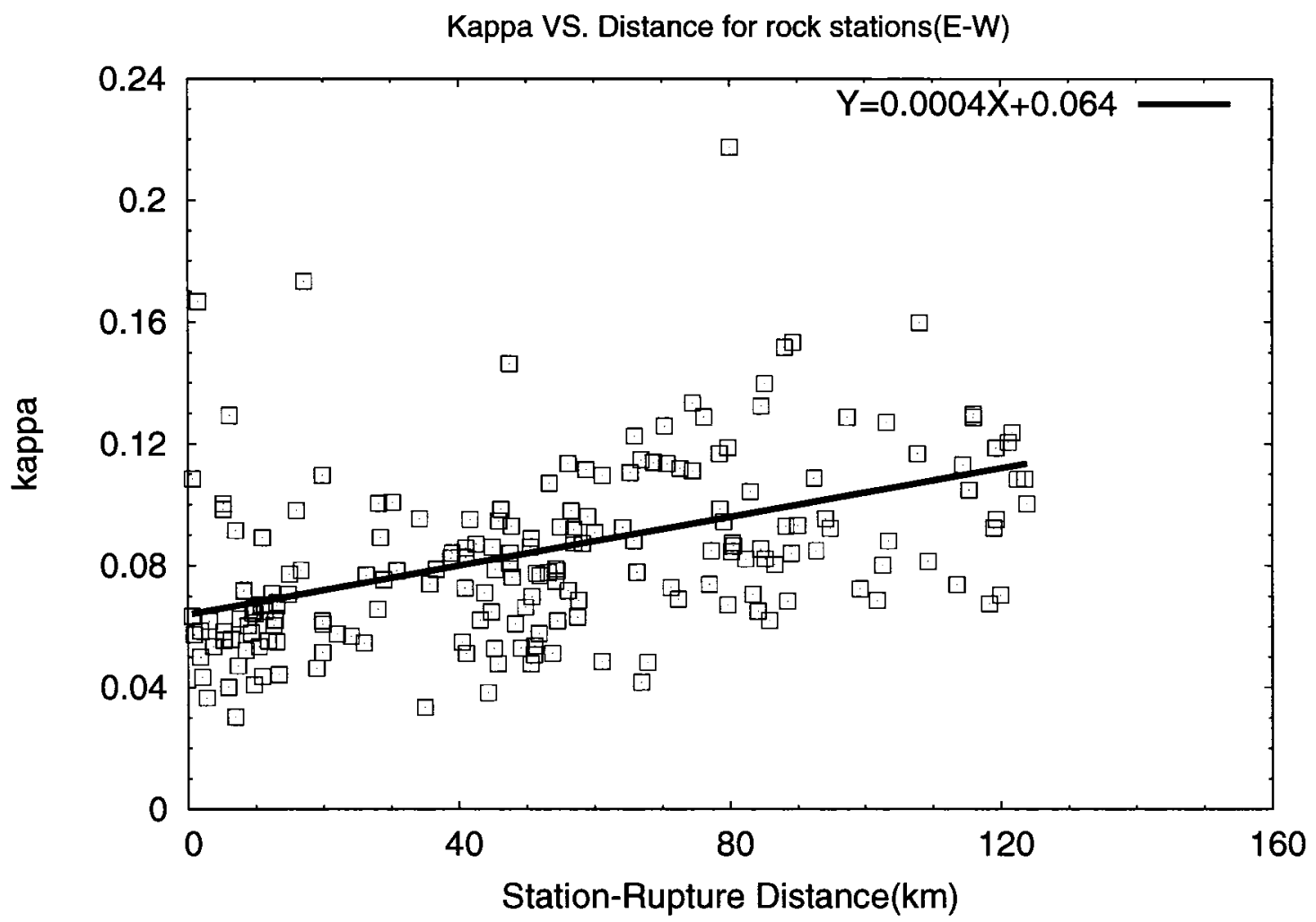

Figure 4.6. $\kappa$ versus station-rupture distance to calculate $\kappa_{0}$ of rock stations (E-W) 


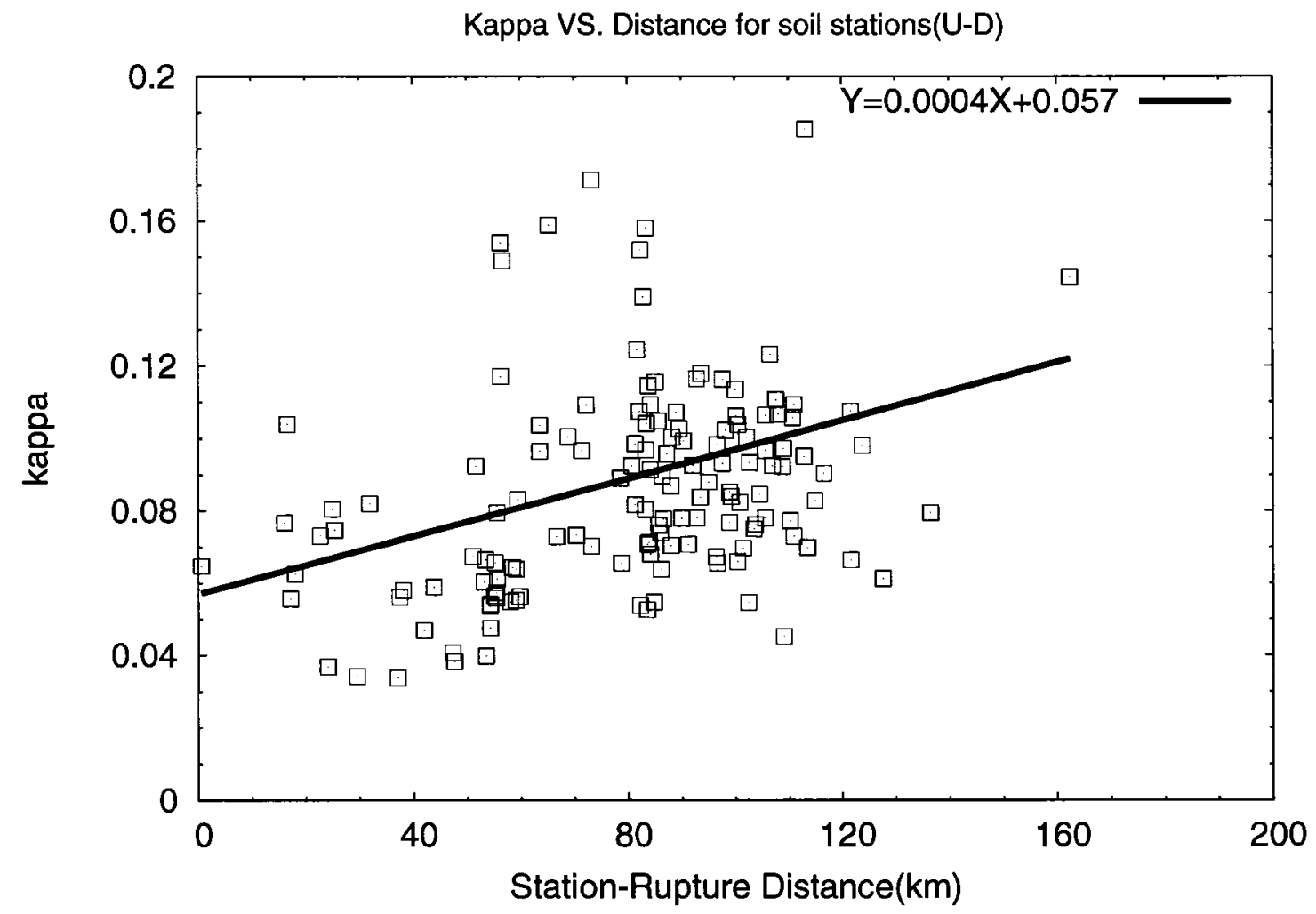

Figure 4.7. $\kappa$ versus station-rupture distance to calculate $\kappa_{0}$ of soil stations (U-D) 


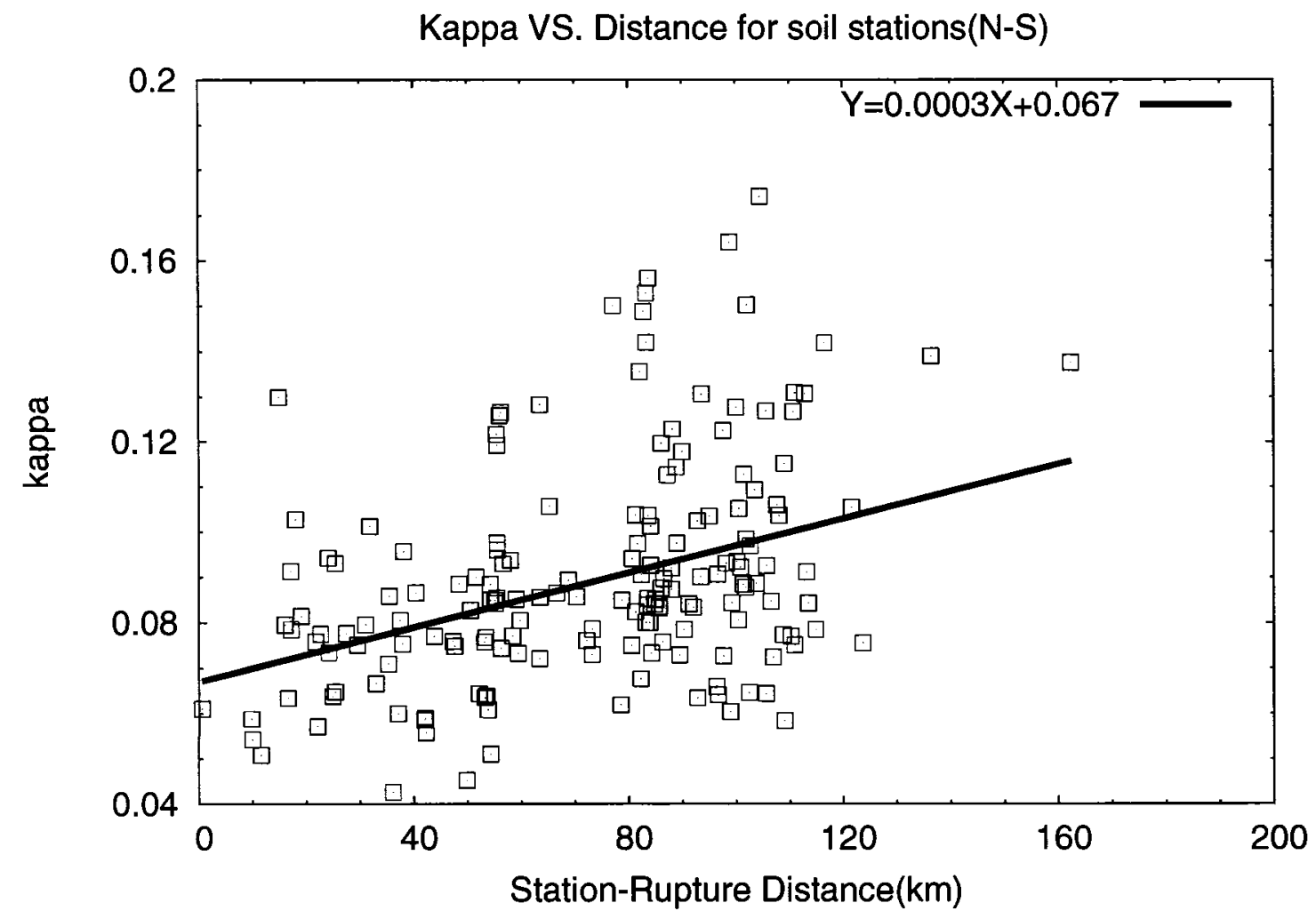

Figure 4.8. $\kappa$ versus station-rupture distance to calculate $\kappa_{0}$ of soil stations (N-S) 


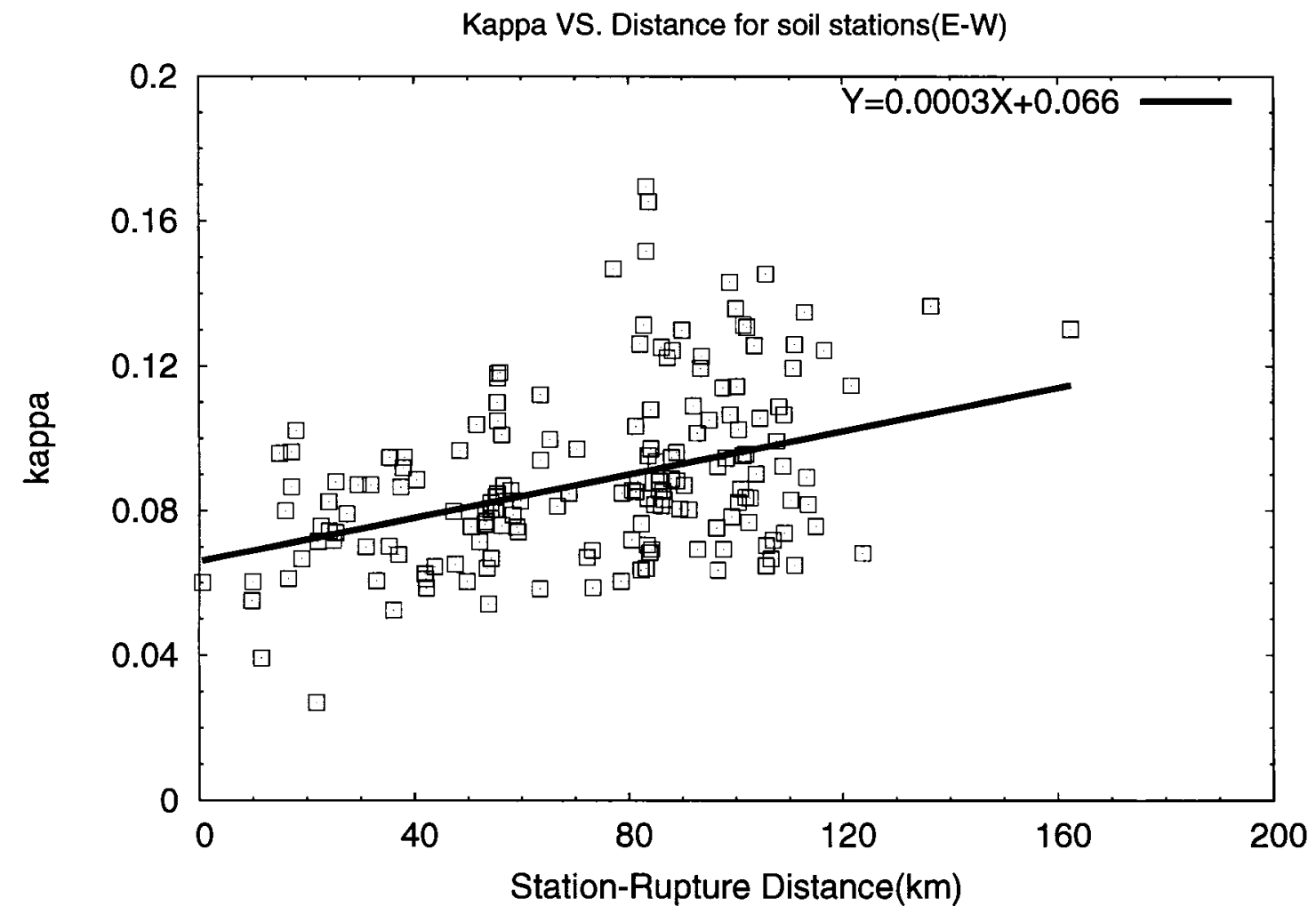

Figure 4.9. $\kappa$ versus station-rupture distance to calculate $\kappa_{0}$ of soil stations (E-W) 

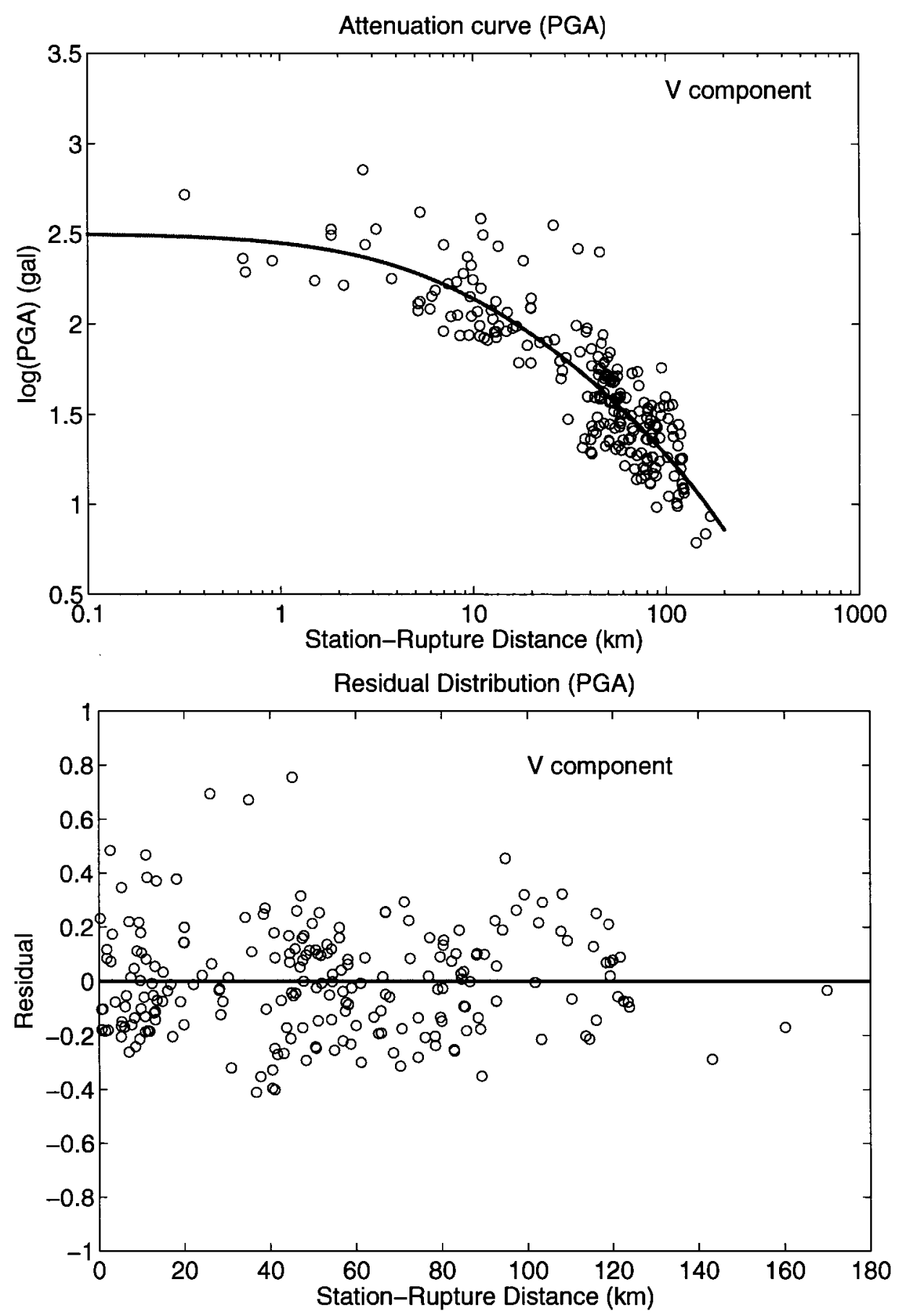

Figure 4.10. (a) Attenuation curve of PGA for rock stations (vertical) (b) Residual distribution of PGA attenuation regression analysis for rock stations (vertical) 

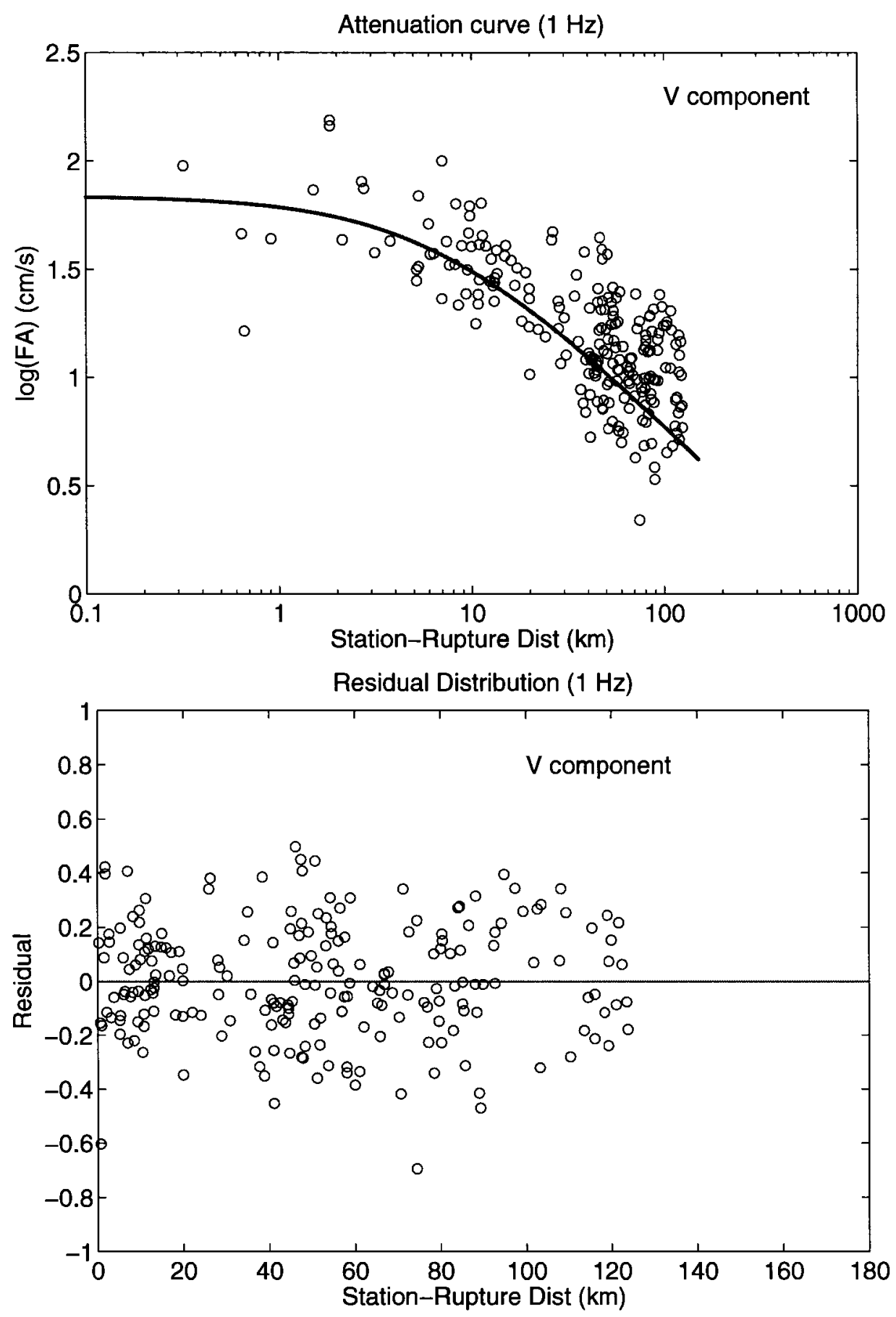

Figure 4.11. (a) Attenuation curve for Fourier acceleration amplitude at $1 \mathrm{~Hz}$ (vertical). (b) Residual distribution of attenuation analysis of Fourier acceleration amplitude at $1 \mathrm{~Hz}$ (vertical) 

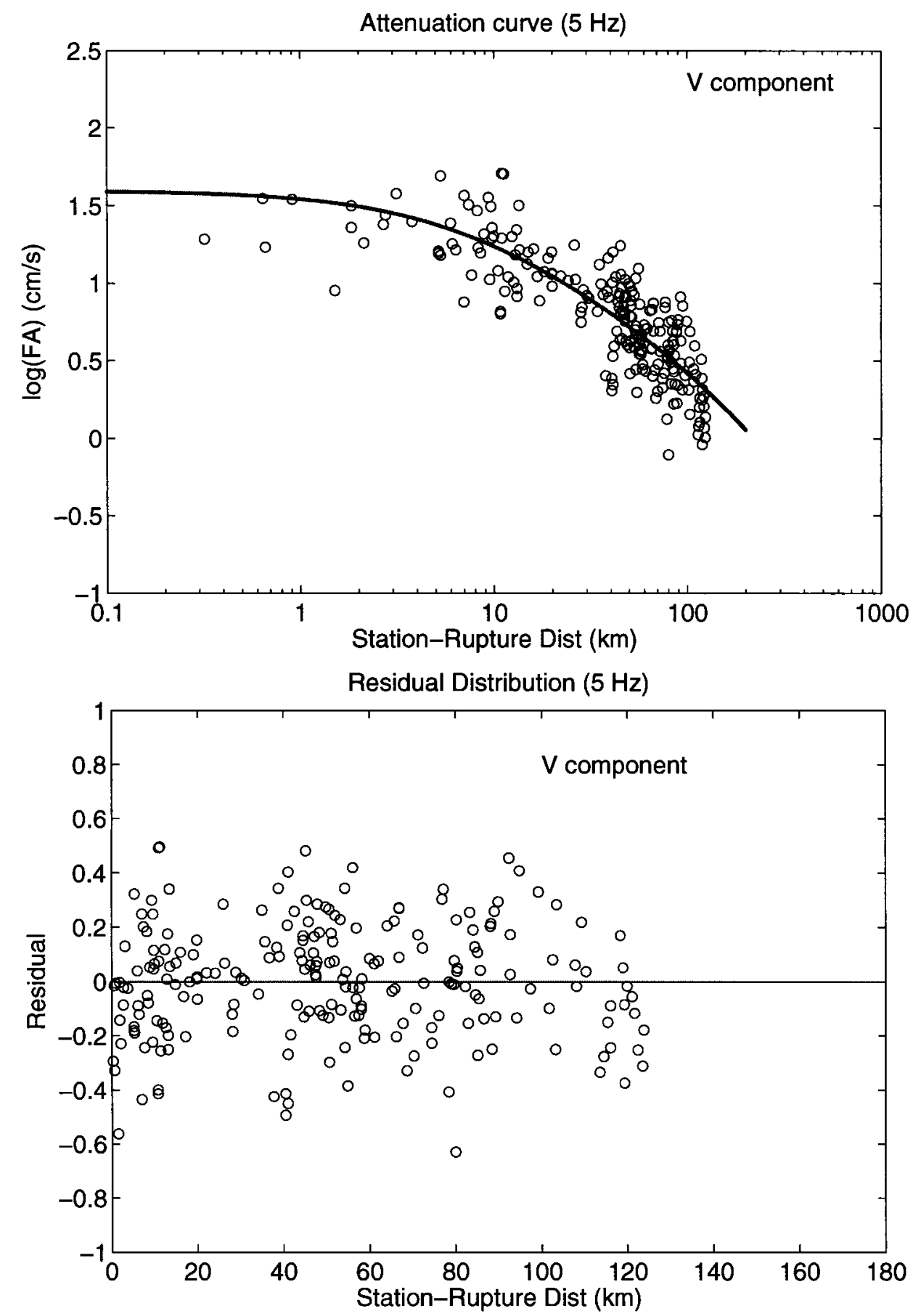

Figure 4.12. (a) Attenuation curve for Fourier acceleration amplitude at $5 \mathrm{~Hz}$ (vertical). (b) Residual distribution of attenuation analysis of Fourier acceleration amplitude at $5 \mathrm{~Hz}$ (vertical) 


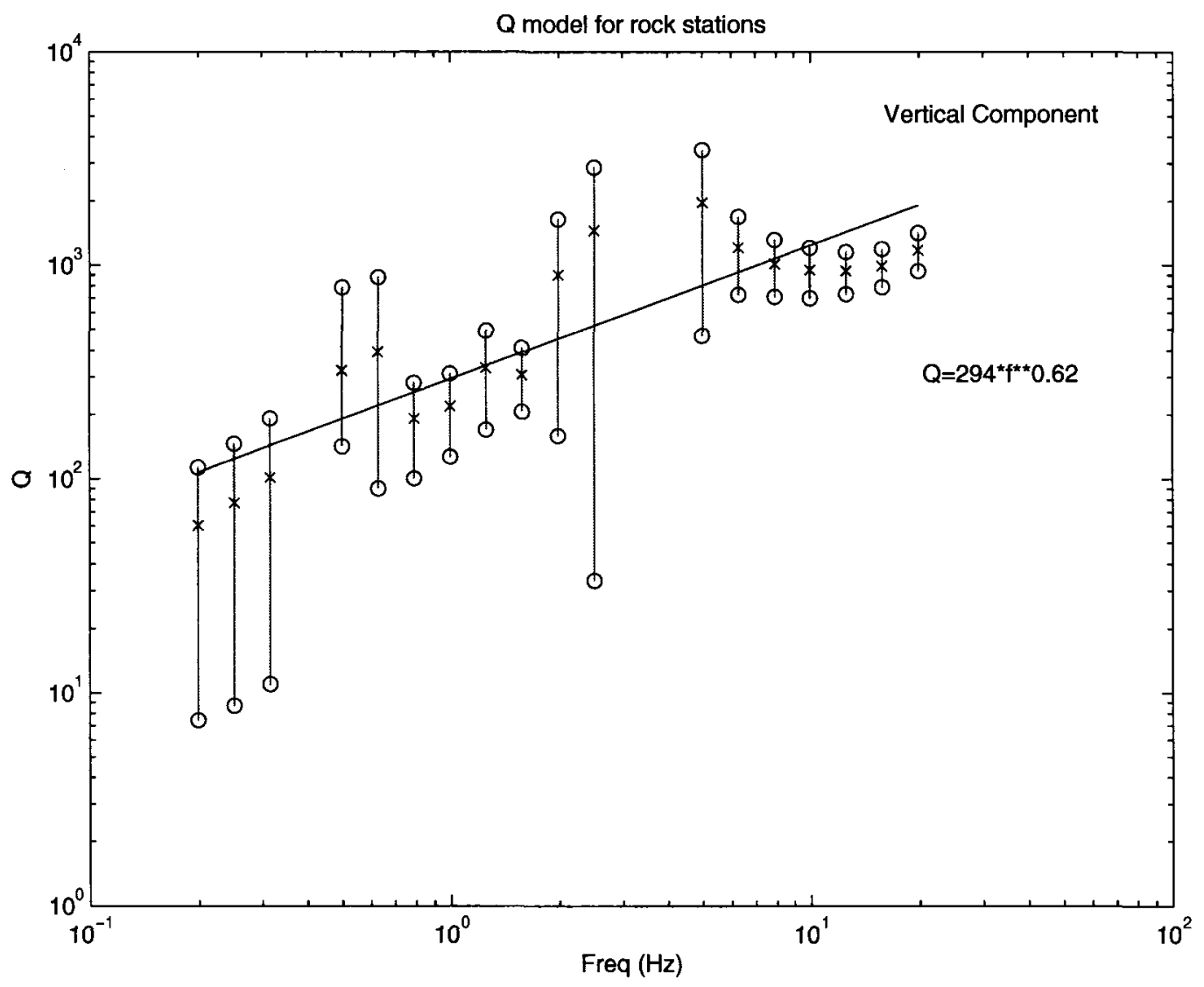

Figure 4.13. Q model; the vertical error bars are 95\% confidence interval 


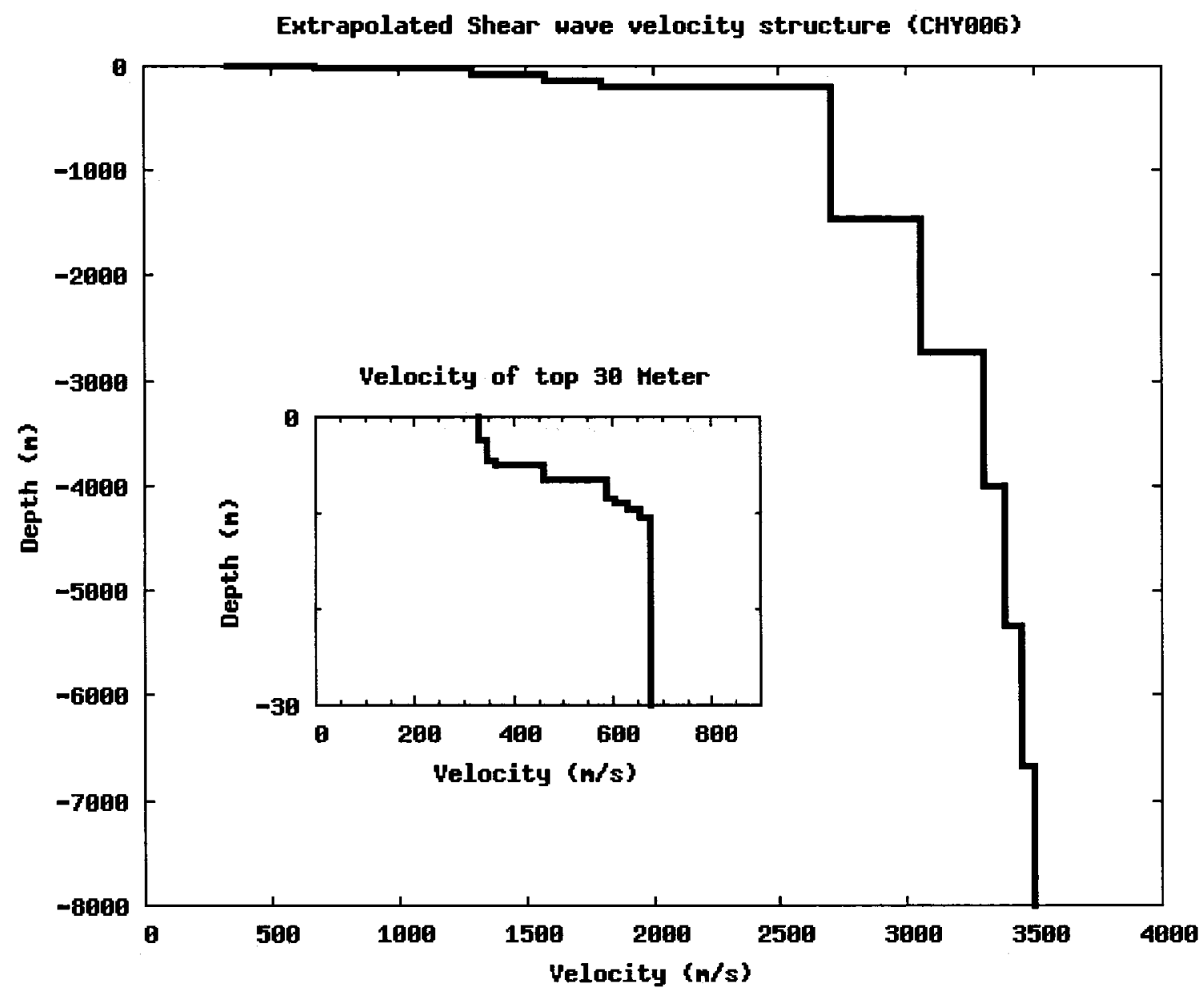

Figure 4.14. The shear wave velocity $\left(V_{s}\right)$ structure used in this study at rock station CHY006 


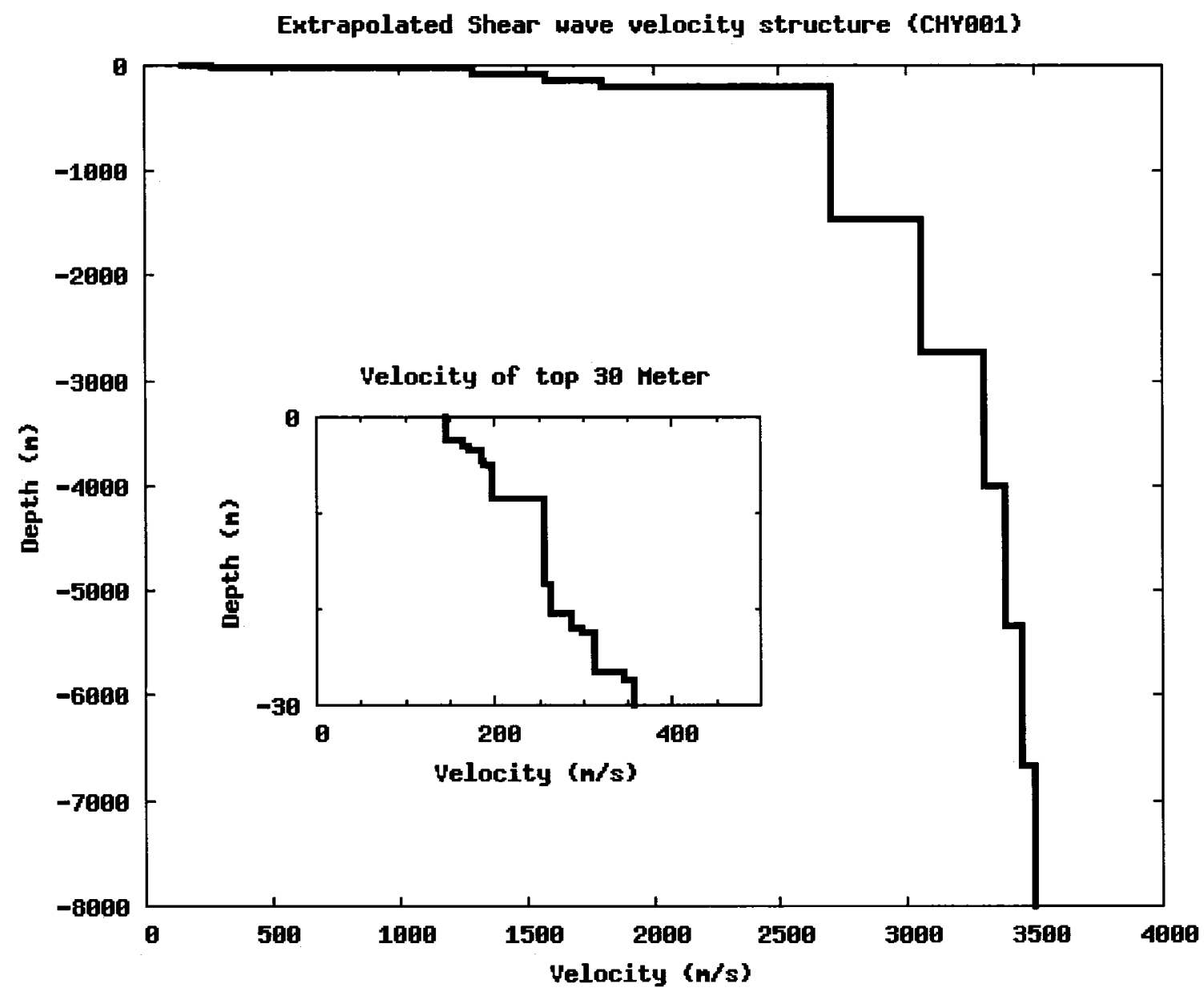

Figure 4.15. The shear wave velocity $\left(V_{s}\right)$ structure used in this study at soil station CHY001 


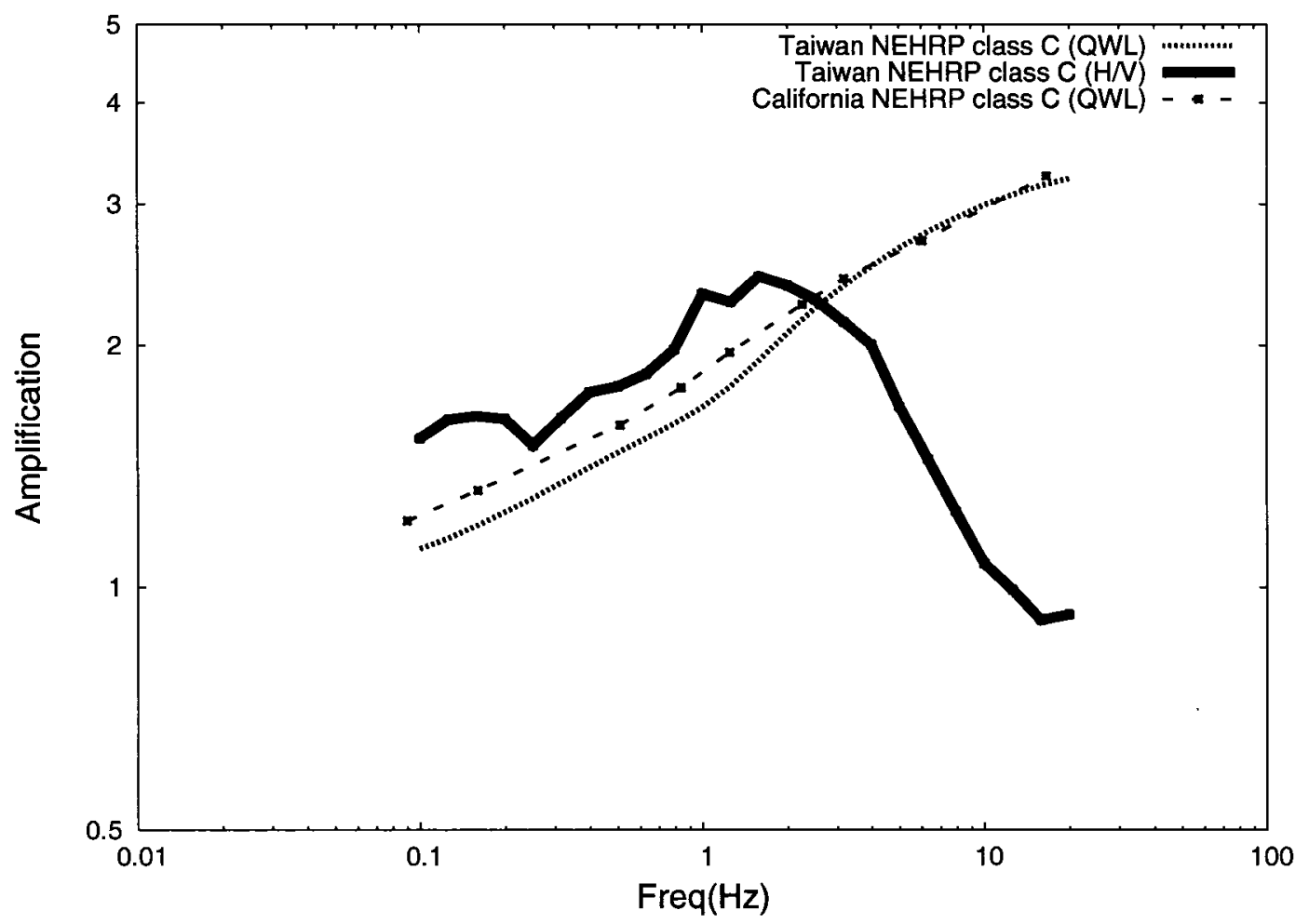

Figure 4.16. Comparison of site amplifications based on $\mathrm{H} / \mathrm{V}$ and quarter wavelength approaches (QWL) for Taiwan and based on quarter wavelength approach (QWL) for California (NEHRP class C) 


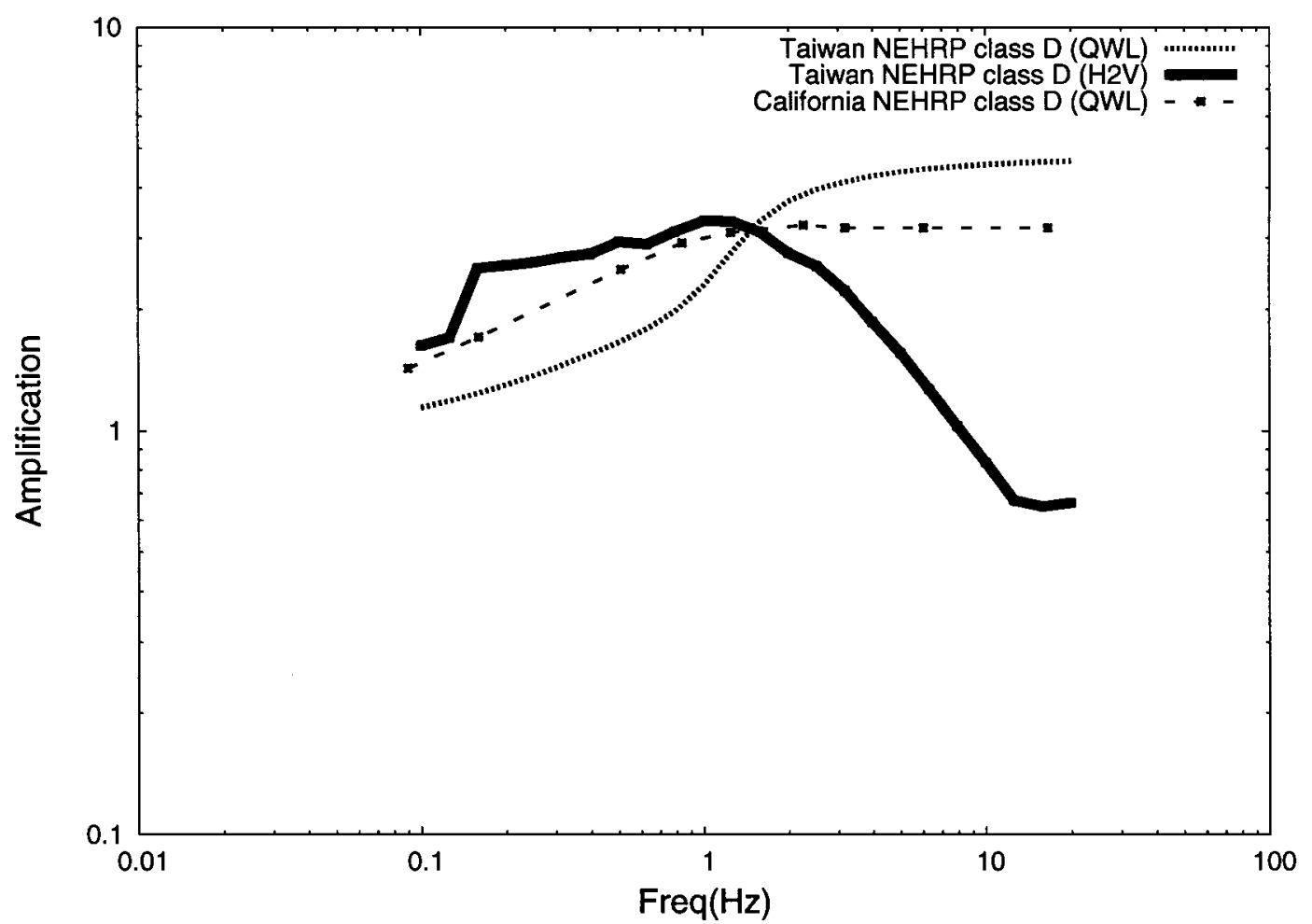

Figure 4.17. Comparison of site amplifications based on $\mathrm{H} / \mathrm{V}$ and and quarter wavelength approaches (QWL) for Taiwan and based on quarter wavelength approach (QWL) for California (NEHRP class D) 


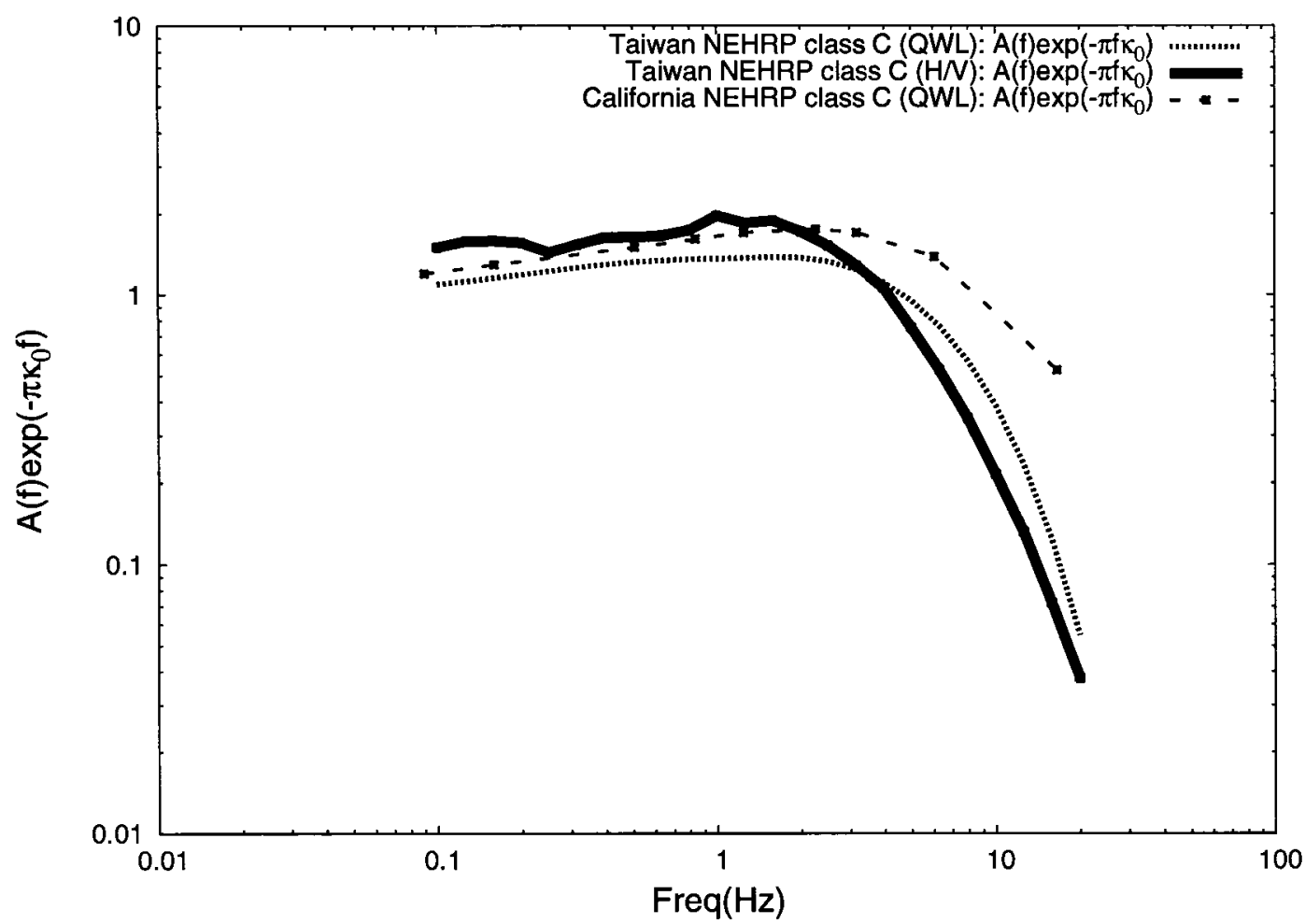

Figure 4.18. Comparison of site term $\left(A(f) \cdot \exp ^{-\pi \kappa_{0} f}\right)$ of NEHRP class $\mathrm{C}$ sites between Taiwan (based on $\mathrm{H} / \mathrm{V}$ combined with $\kappa_{0 V}=0.051$ and quarter wavelength approximation (QWL) combined with $\kappa_{0 H}=0.064$ ) and California (based on quarter wavelength approximation (QWL) combined with $\kappa_{0 H}=0.035$ ) 


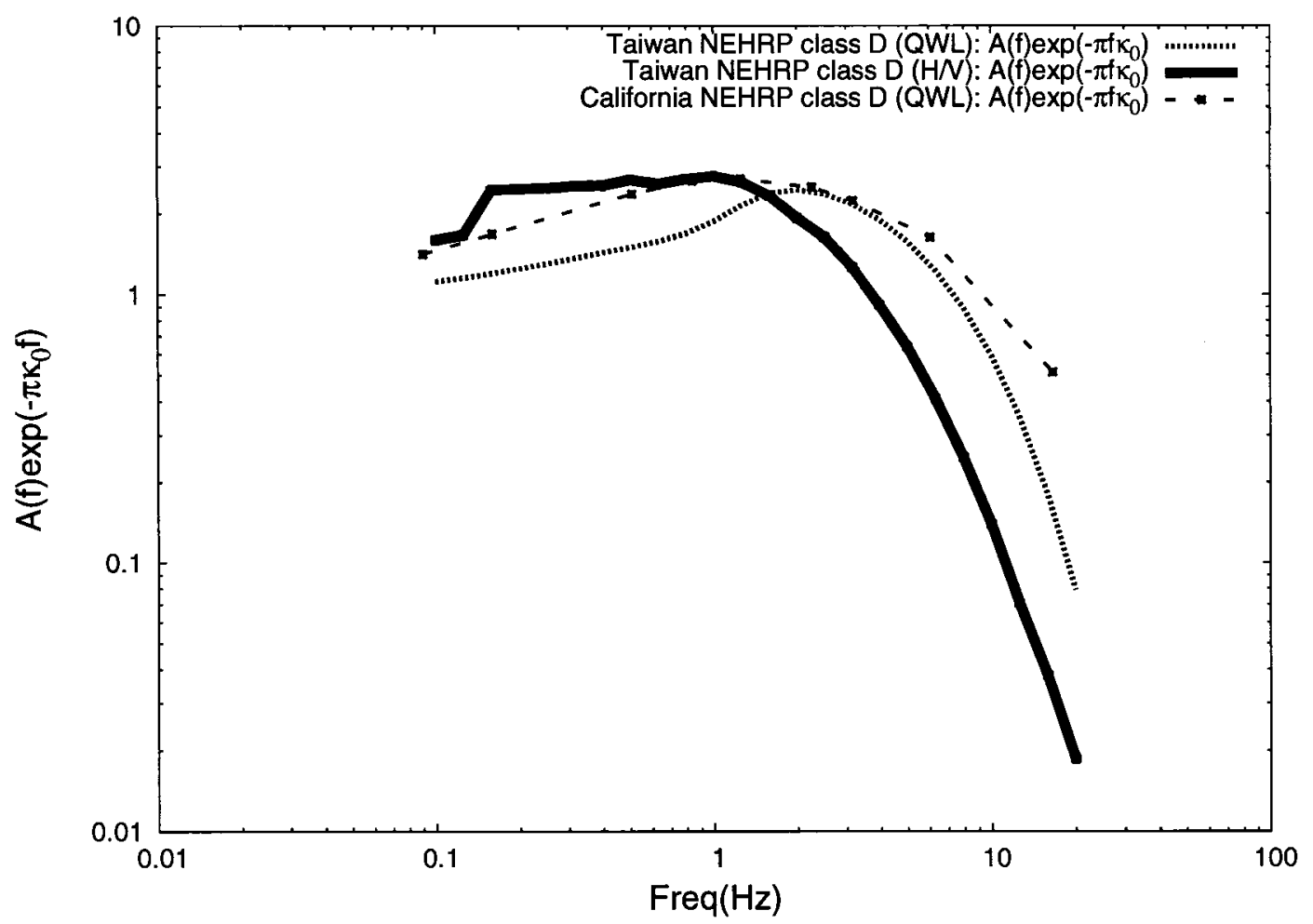

Figure 4.19. Comparison of site term $\left(A(f) \cdot \exp ^{-\pi \kappa_{0} f}\right)$ of NEHRP class D sites between Taiwan (based on $\mathrm{H} / \mathrm{V}$ combined with $\kappa_{0 V}=0.057$ and quarter wavelength approximation (QWL) combined with $\kappa_{0 H}=0.066$ ) and California (base on quarter wavelength approach (QWL) combined with $\left.\kappa_{0 H}=0.035\right)$ 
Duration of motions for rock stations

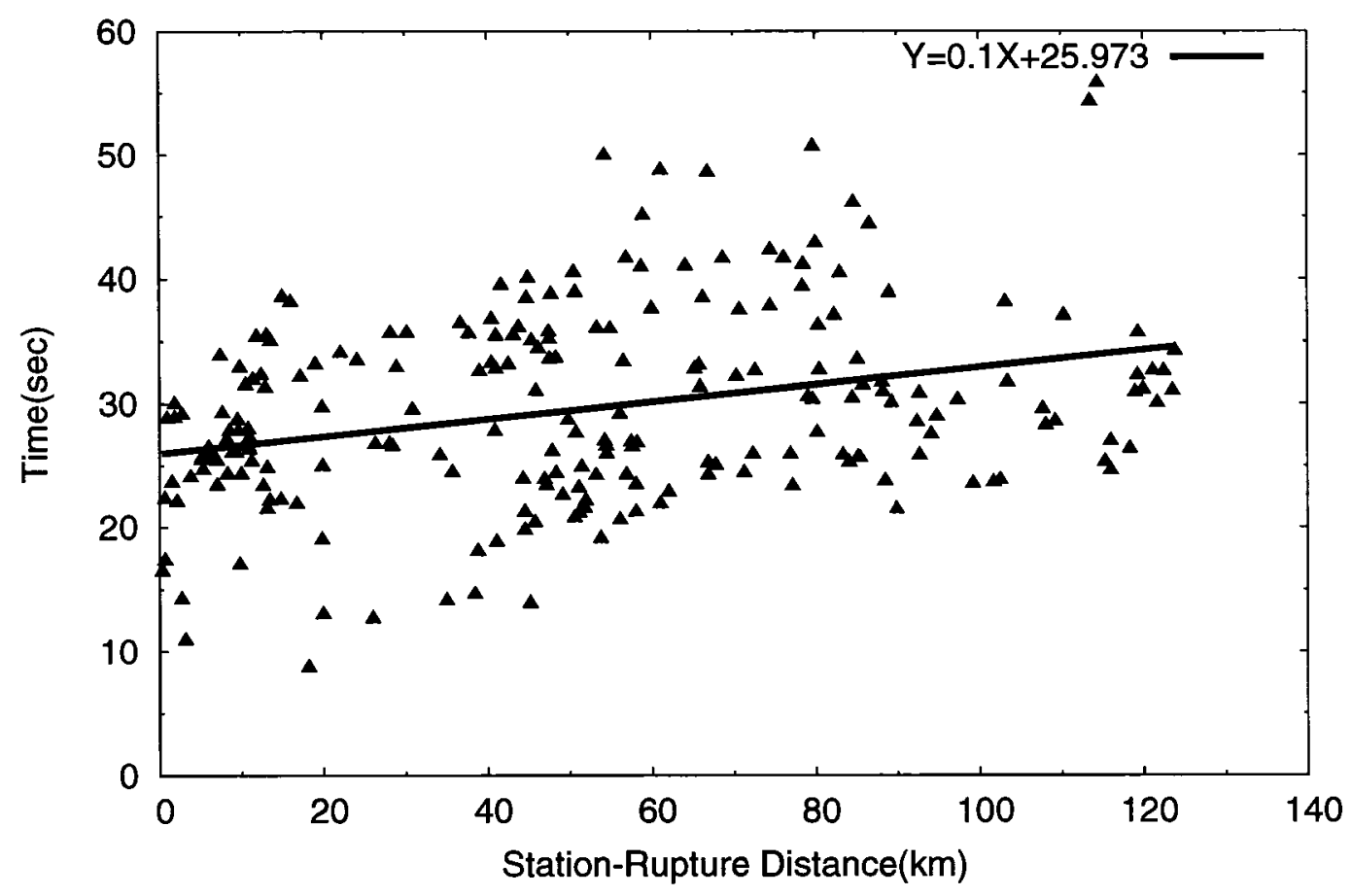

Figure 4.20. Duration of motions in the Chi-Chi mainshock 


\section{Chapter 5}

\section{SIMULATION OF THE CHI-CHI EARTHQUAKE GROUND MOTION}

\subsection{Introduction}

After obtaining all of the needed input parameters for the stochastic simulation, the following steps are used to finalize the simulation. First, I use the rock stations (NEHRP class C) as reference stations to calibrate the input model: that is, to determine two "free parameters" stress drop and pulsing percentage, which can not be directly derived from the seismic records. These parameters are determined based on the minimization of root mean square of residuals $\left(\mathrm{rms}=\sqrt{\frac{\sum_{i=1}^{N}\left(r_{i} \cdot r_{i}\right)}{N}}\right.$, where $\mathrm{r}$ is the model residual for each frequency bin and $\mathrm{N}=24$ is the number of frequency bin). Then, the estimated stress drop and pulsing percentage are applied to the soil stations to analyze the possible non-linear soil response.

Finally, the soil stations located in the Taipei metropolitan area are selected to analyze the effect of the Taipei basin on ground motions.

\subsection{Input Model}

All of the input parameters for the simulations are listed in Table 5.1. Fault geometrical parameters and other physical parameters are as discussed in Chapter 4. Only two "free" parameters, stress drop and pulsing percentage, are unknown and should be calibrated. I vary the stress drop from 20 to 100 bars in 10 bar steps, while the pulsing percentage is fixed at $50 \%$ to search for the result that produces 
minimum rms of residuals where residual is defined as $\log \left(\mathrm{PSA}_{\mathrm{obs}}\right)_{i}-\log \left(\mathrm{PSA}_{\text {sim }}\right)_{i}$. $\mathrm{PSA}_{\mathrm{obs}}$ is the arithmatic average of the E-W and N-S components PSA at the $i^{\text {th }}$ station. The pulsing percentage does not affect the stress drop dramatically; thus, the pulsing percentage can be fixed in the determination of stress drop. (The stress drop determines the high frequency level of ground motion while pulsing percentage affects low frequencies only.)

Table 5.1: Input model parameters for the extended finite fault stochastic simulation

\begin{tabular}{|c|c|}
\hline Fault dimension $(\mathrm{km})$ & $70 \times 40$ \\
\hline Subfault dimension $(\mathrm{km})$ & $6.4 \times 5$ \\
\hline Depth of fault $(\mathrm{km})$ & 0 \\
\hline Fault orientation & strike: $5^{\circ}$, dip $: 34^{\circ}$ \\
\hline Mainshock moment magnitude $\left(M_{W}\right)$ & 7.6 \\
\hline Hypocenter location (subfault) & 4 \\
\hline$V_{s}(\mathrm{~km} / \mathrm{s})$ & 3.2 \\
\hline Density $\left(\mathrm{g} / \mathrm{cm}^{3}\right)$ & 2.7 \\
\hline Stress parameters (bars) & $\begin{array}{l}\text { increase from } 20 \text { to } 100 \\
\text { with step } 10\end{array}$ \\
\hline Pulsing percentage & $\begin{array}{l}\text { fix as } 50 \% \text { to determine stress drop } \\
\text { then increase from } 10 \% \text { to } 90 \% \text { in } 10 \% \text { steps }\end{array}$ \\
\hline Windowing function & Saragoni-Hart \\
\hline Geometric spreading & -0.9 \\
\hline $\mathrm{Q}(\mathrm{f})$ & $Q=294 \cdot f^{0.61}$ \\
\hline Duration & $T=T_{0}+d \cdot R($ Atkinson and Boore, 1995) $\mathrm{d}=0.1$ \\
\hline Kappa operator $(\kappa)$ & 0.065 \\
\hline Amplification & NEHRP class C (quarter wavelength approximation) \\
\hline Site classification & NEHRP class $\bar{C}$ \\
\hline Slip model & random slip distribution \\
\hline
\end{tabular}




\subsection{Results and Analysis}

In the following sections, simulation based on the parameters shown in Table 5.1 is conducted for rock stations to calibrate the model and then applied to soil stations investigating the non-linear soil and the basin responses.

\subsubsection{Simulation for Rock Stations}

The averaged residual for models with different stress drop (from 20 bars to 100 bars with 10 bars increment) but fixed pulsing percentage (50\%) are plotted together in Figure 5.1 to find the average stress drop in the 1999 Chi-Chi mainshock. According to Figure 5.1, the stress drop of the mainshock is between 30 and 50 bars. After determining the stress drop range, the combined effect of the stress drop and the pulsing percentage are evaluated by varying the stress drop from 30 bars to 50 bars (with 5 bars increment) and pulsing percentage from $10 \%$ to $90 \%$ (with step $10 \%$ ). Table 5.2 shows the comparison of the model bias with different stress drop and pulsing percentages. According to the model bias shown in Table 5.2 the stress drop is about 40 bars and the pulsing percentage is above $80 \%$ (The average model bias and the root mean square of the model bias are the smallest). 
Table 5.2 Comparison of Model bias for different stress drop and pulsing percentage combination

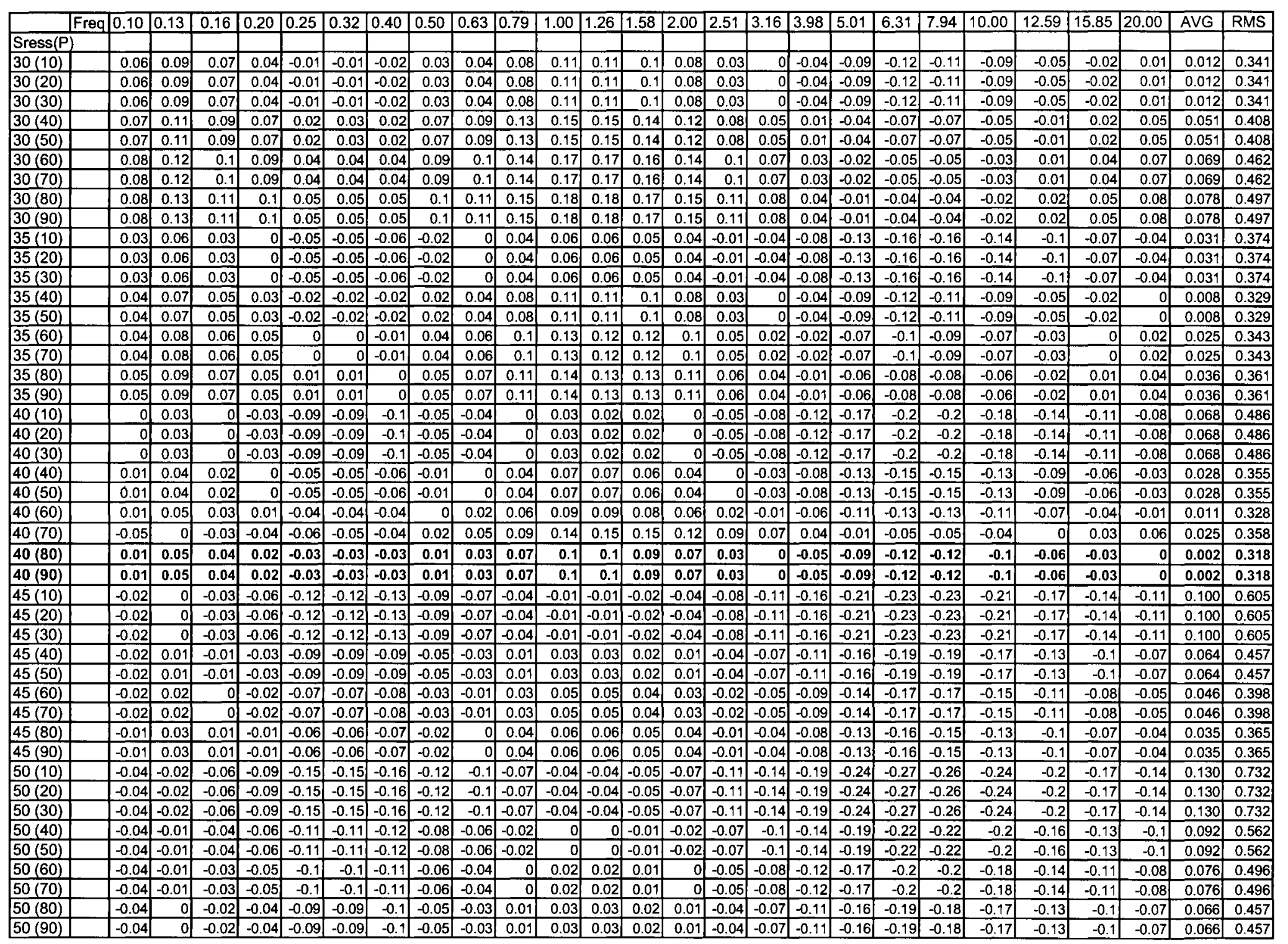

INote: AVG is the arithmatic average model bias for all interested frequency span

RMS is the root mean square defined as $\checkmark\left(\sum\left(r^{*} r\right)\right) / N$ where $r$ is the model residual for each frequency and $N$ is the number of frequency bin 
Figure 5.2 shows the average model bias $\log \left(\mathrm{PSA}_{\mathrm{obs}}\right)-\log \left(\mathrm{PSA}_{\text {sim }}\right)$ versus frequency for the calibrated model (stress drop $=40$ bars and pulsing percentage $=80 \%$ ). For the frequency below $0.8 \mathrm{~Hz}$, the model fits the recorded data very well, showing the near zero mean residual. However, the overall residual between $0.8 \mathrm{~Hz}$ and $2 \mathrm{~Hz}$ is positive, showing a minor underestimate of the simulated ground motion due to the possible underestimation of the site amplification function (the dominant contributing factor for the underestimation is probably the basin effect around $1 \mathrm{~Hz}$ in Taipei basin area, which is discussed in detail later). The residual between $4 \mathrm{~Hz}$ and $10 \mathrm{~Hz}$ is negative, demonstrating overestimation from the input model. From another point of view, Figure 5.3 shows the residual distribution over distance for $0.5 \mathrm{~Hz}$ and Figure 5.4 shows the residual distribution for $1 \mathrm{~Hz}$. Figure 5.5 and Figure 5.6 are the residual distribution for $5 \mathrm{~Hz}$ and $10 \mathrm{~Hz}$, respectively. Figure 5.3 has zero mean residual and no trend over distance. Figure 5.4 has positive average residual (about 0.1) but no obvious trend over distance. The positive upward shift of the residual for $1 \mathrm{~Hz}$ is reasonable. Figure 5.5 and Figure 5.6 both show a downward shift of the model bias but no obvious trend over distance. There is no noticeable trend in the model residual distribution over distance indicating that the attenuation model chosen in this study is reasonable.

Apart from the random slip distribution model (generated randomly by the computer program) used in the above model calibration, the pre-determined slip distribution model by Wang et al. (2001) is also used in the simulation to investigate the effect of the rupture process on the simulation. Figure 5.7 is the model bias over frequency, showing a slight downward shift compared with the residual distribution of the random slip distribution model. However, only minor differences appear between Figure 5.2 and Figure 5.7, demonstrating that there is little effect of the rupture pro- 
cess on the overall model bias using the finite fault stochastic simulation technique; this was also pointed out by Beresnev and Atkinson (1998b). Meanwhile, the residual distribution versus distance at $0.5 \mathrm{~Hz}$ (Figure 5.8), $1 \mathrm{~Hz}$ (Figure 5.9), 5Hz (Figure 5.10) and $10 \mathrm{~Hz}$ (Figure 5.11) are plotted to investigate the effect of the pre-determined slip model on the model bias, especially on the near source records, which may be significantly controlled by the directivity effect. The above residual distribution versus distance figures of the pre-determined slip model show such effect, especially in the high frequency. Figure 5.12 and Figure 5.13 compare the map view of the model bias for the random slip and prescribed slip models at $1 \mathrm{~Hz}$ and $10 \mathrm{~Hz}$, respectively. Generally, these two figures only show some minor difference (the overall bias difference between random slip and pre-determined slip model is small, as discussed above). However, for the near-fault region, the difference is noticeable indicating the possible effect of the slip distribution model on the near-fault stations, especially at high frequency.

The $\mathrm{H} / \mathrm{V}$ ratio is also used in the simulation to compare the accuracy of the $\mathrm{H} / \mathrm{V}$ ratio method and the quarter wavelength approximation approach. Parameters used in the simulations remain the same as that in Table 5.1 except $\kappa_{0}$ is set as $\kappa_{0 V}$ and the site amplification is the $\mathrm{H} / \mathrm{V}$ ratio. Thus, the overall site term is $A(f)_{H / V} \cdot \exp ^{-\pi \kappa_{0 V} f}$. Figure 5.14 compares the model bias versus frequency using the $\mathrm{H} / \mathrm{V}$ ratio as the amplification function and the quarter-wavelength estimates as site amplification; this indicates that there is little difference between the two approaches. For low frequencies, there is a downward shift for the $\mathrm{H} / \mathrm{V}$ ratio model compared with the quarter wavelength approximation model because the site amplification effects derived from the $\mathrm{H} / \mathrm{V}$ ratio method is larger than that from the quarter wavelength approximation approach (See Figure 4.18). Figure 5.15 and Figure 5.16 are the bias versus distance 
at $1 \mathrm{~Hz}$ and $5 \mathrm{~Hz}$ showing the similar distribution pattern as that in the calibrated model. All in all, the quarter wavelength approximation method is preferred to calculate the site amplification function but it needs the detailed shear wave velocity information for the research region (in most cases, such information is not available). The $\mathrm{H} / \mathrm{V}$ ratio shows similar applicability as the quarter wavelength approximation approach, according to this study, but it is much more economical than the quarter wavelength approximation method. It could be a suitable candidate for the site amplification calculation when the shear wave velocity information is not available for the research area.

\subsubsection{Simulation for linear behavior of soil stations}

In this step, the simulation is applied to soil stations (NEHRP class D sites) with the obtained stress drop (40 bars), the pulsing percentage (80\%) and other listed parameters in Table 5.1. Different site amplification schemes are used; one based on the quarter wavelength approximation approach and another based on the $\mathrm{H} / \mathrm{V}$ ratio.

\section{Simulation for soil stations using quarter wavelength approximation as} site amplification First, site amplification for NEHRP class D stations calculated by the quarter wavelength approximation method is used in simulations. Figure 5.17 shows the residual distribution over frequency, indicating an underestimation at low frequency but overestimating of ground motions at high frequency. Figure 5.18 and Figure 5.19 plot the residual distribution versus distance at frequency $0.5 \mathrm{~Hz}$ and 2 $\mathrm{Hz}$, and show a noticeable upward trend over distance. This might be a manifestation of nonlinear site response, as it indicates relative overestimation of near-source amplitudes at high frequencies. 
Simulation for soil stations using $H / V$ ratio as site amplification $H / V$ ratio combined with the vertical kappa operator $\left(\kappa_{0 V}=0.057\right)$ for NEHRP class D stations, may also be used as the site amplification function in simulations. The residual versus frequency graph (Figure 5.20) shows an improvement compared with Figure 5.17. However, Figure 5.21 and Figure 5.22 still shows evidence of the nonlinear soil response in the Chi-Chi mainshock with the $\mathrm{H} / \mathrm{V}$ ratio as site amplification, but the effect is smaller than that of quarter wavelength approximation, shown in Figure 5.17. This suggests that the nonlinearity may be similar on both vertical and horizontal components. Thus, degree of most evidence of nonlinearity is cancelled out in the $\mathrm{H} / \mathrm{V}$ method.

\subsubsection{Simulation for non-linear behavior of soil stations}

Background theory The above discussion witnesses the possible nonlinear behavior of the soil stations. Thus, in the following session, the nonlinear soil response correction method based on empirical analysis of other earthquakes (Choi and Stewart, 2005) is used and the general methodology of the non-linear correction approach is discussed in detail. Basically, the nonlinear correction approach is based on Equation 5.1:

$$
\mathrm{PSA}_{\text {corrected }}=\mathrm{PSA}_{\text {simulated }} \cdot \exp \left(b_{\text {cor }}\right)
$$

where PSA corrected $_{\text {is }}$ PSA after correction for nonlinearity, PSA $_{\text {simulated }}$ is the simulated PSA without correction (eg. ground motion simulated at the reference site condition, NEHRP class C, using the calibrated model parameters and then corrected to the desired soil station condition using the correction factor described below). $b_{c o r}$ is the correction factor (Equation 5.2 and Equation 5.3) which comprises the linear amplification correction factor and an empirical nonlinear amplification correction 
factor derived from a large strong-motion database (Boore and Atkinson, 2005).

$$
b_{\text {cor }}=b_{\text {lin }} \ln \left(v_{30} / v_{r e f}\right)+b_{n l} \ln (0.06 / 0.1) \quad \text { for } \quad \operatorname{pga} 4 \mathrm{nl}<=0.06 g
$$

and

$$
b_{c o r}=b_{l i n} \ln \left(v_{30} / v_{r e f}\right)+b_{n l} \ln (\mathrm{pga} 4 \mathrm{nl} / 0.1) \quad \text { for } \quad \mathrm{pga} 4 \mathrm{nl}>0.06 g
$$

where $b_{l i n}$ is the linear correction coefficient, $v_{30}$ is the average shear wave velocity of the upper 30 meter layer at stations, $v_{r e f}$ is the reference rock station (In this study, $v_{r e f}=500 \mathrm{~m} / \mathrm{s}$ because $\bar{v}_{30}$ of NEHRP class C sites is equal to $500 \mathrm{~m} / \mathrm{s}$ in Taiwan). $b_{l i n}$ used in this study is published by Choi and Stewart (2005) based on the empirical analysis of many earthquakes (similar linear coefficients were also published by Boore et al. (1997) and their comparison is shown in Figure 5.23). $b_{n l}$ is the nonlinear correction coefficient and the pga4nl is the threshold of the peak ground acceleration above which the nonlinear response occurs. The pga4nl value can be obtained by Equation 5.8 (Boore and Atkinson, 2005). $b_{n l}$ could be calculated by Equation 5.4 to 5.7 (Boore and Atkinson, 2005).

$$
\begin{aligned}
& b_{n l}=b_{1} \text { for } v_{30}<=v_{1} \\
& b_{n l}=\left(b_{1}-b_{2}\right) \ln \left(v_{30} / v_{2}\right) / \ln \left(v_{1} / v_{2}\right)+b_{2} \text { for } v_{1}<v_{30}<=v_{2} \\
& b_{n l}=b_{2} \ln \left(v_{30} / v_{r e f}\right) / \ln \left(v_{2} / v_{r e f}\right) \text { for } v_{2}<v_{30}<=v_{r e f} \\
& b_{n l}=0 \text { for } v_{30}>v_{r e f}
\end{aligned}
$$

where $b_{1}, b_{2}$ are regression coefficients empirically determined by Choi and Stewart (2005) (See Table 5.3 for details), $v_{1}, v_{2}$ are the boundary of the piecewise function, $v_{30}$ is the average shear wave velocity of the upper 30 meter at stations, $V_{\text {ref }}$ is the 
shear wave velocity of the reference rock station (Boore and Atkinson, 2005).

$$
\log (\text { pga4nl })=2.573-0.55 \log (r / 5)-0.005(r-5)+0.241 \text { eps } \quad \text { for } \quad M_{W}>7.0
$$

where $r=\sqrt{r_{j b}^{2}+3.0^{2}}, r_{j b}$ is the Joyner-Boore distance and eps is the number of sigmas ( $=0$ for median PGA) and PGA is in $\mathrm{cm} / \mathrm{s} / \mathrm{s}$ (Boore and Atkinson, 2005).

In the following section, firstly, the empirical correction just for the linear case by Boore and Atkinson (2005) from NEHRP class C to NEHRP class D stations will be discussed and then compared with the above two simulations (Quarter wavelength approximation and $\mathrm{H} / \mathrm{V}$ on NEHRP class $\mathrm{D})$. Secondly, the nonlinear correction scheme based on Boore and Atkinson (2005) are applied to the simulations.

When the simulated PGA value at the station is below the nonlinear threshold $(0.06 \mathrm{~g}$ in this study), only linear correction is considered. Otherwise, the nonlinear correction is applied. Figure 5.24 and Figure 5.25 demonstrate the variation of the linear correction term $\left(b_{\text {cor }(l i n)}\right)$, the nonlinear correction term $\left(b_{c o r(n l)}\right)$ and the combined effect of the linear and the nonlinear corrections for two different PGA values $(0.05 \mathrm{~g}$ and $0.15 \mathrm{~g})$, respectively. Figure 5.24 shows the linear correction effect because there is no nonlinearity effect for PGA below 0.06g. In Figure 5.25, the linear, nonlinear correction terms and their combination are plotted $(\mathrm{PGA}=0.15 \mathrm{~g})$. Furthermore, the exponential form of the correction term $\left(\exp ^{b_{c o r}}\right)$ is shown in Figure 5.26 for $\mathrm{PGA}=0.15 \mathrm{~g}$.

In this section, the above correction terms are applied in the site amplification term for the simulations. First, ground motions at soil stations are generated using the NEHRP class $\mathrm{C}$ site amplification (at reference class $\mathrm{C}$ site) calculated by quarter wavelength method. Then, only the linear correction term is applied to correct the ground motions which are simulated on reference stations to the desired site conditions 
(In this case, it is to eliminate the difference between NEHRP class C stations and NEHRP class D stations based on the empirical analysis using Equation 5.2). For stations whose PGA is smaller than the nonlinearity threshold $(0.06 \mathrm{~g})$, nonlinear correction has no effect on the ground motions ( $\exp ^{b_{c o r}}$ is equal to 1 ); while for other stations, nonlinear correction is used to remove the effects of soil nonlinearity.

\section{Empirical linear correction between NEHRP class $C$ and NEHRP class}

D stations Figure 5.27 shows the model residual distribution versus frequency for the simulated motions with only the linear correction. It shows the near zero residual from low to intermediate frequencies but an overestimation of ground motions at high frequency. Figure 5.28, Figure 5.29, Figure 5.30 and Figure 5.31 are residual distribution over distance at frequency $0.5 \mathrm{~Hz}, 2 \mathrm{~Hz}, 5 \mathrm{~Hz}$ and $10 \mathrm{~Hz}$. These graphs also suggest soil nonlinearity at near source stations. Then, in the next step, nonlinear correction is added to the site term and its effects on the near-source stations are illustrated in Figure 5.33 to Figure 5.36.

\section{Result of nonlinear correction based on Boore and Atkinson (2005)} The nonlinear correction approach uses equation 5.4 to 5.7 to calculate the nonlinear correction coefficient $b_{n l}$ (Boore and Atkinson, 2005). Figure 5.32 shows the model residual distribution over frequency for the nonlinear correction scheme proposed by Boore and Atkinson (2005). Figure 5.33, Figure 5.34, Figure 5.35 and Figure 5.36 show the model bias distribution versus distance and its least square regressed trend at $0.5 \mathrm{~Hz}, 2 \mathrm{~Hz}, 5 \mathrm{~Hz}$ and $10 \mathrm{~Hz}$, respectively. Comparison between model residual distribution before nonlinear correction (only linear correction is applied) in Figure 5.28- 5.31 and after nonlinear correction (linear correction and nonlinear correction are both applied) in Figure 5.33- 5.36 clearly shows the nonlinear behaviour of 
soil sites in the near-fault region.

In conclusion, comparing Figure 5.32 with Figure 5.17 and according to the simulation results of the soil stations based on the above four models, we may conclude that:

1. There is nonlinear soil behavior at near-fault stations during the Chi-Chi mainshock based on site amplification modeling for NEHRP class D sites (quarter wavelength approximation and $\mathrm{H} / \mathrm{V})$.

2. The empirical linear correction term performs well for low and intermediate frequencies but shows overestimation of ground motions at high frequency. It also shows the soil nonlinearity effect during the Chi-Chi mainshock.

3. The nonlinear correction scheme based on Boore and Atkinson (2005) shows a noticeable correction effect in the near source stations, but for the far field, there is an over-correction trend.

Basin Effects As noticed by Fletcher and Wen (2005), the PGA and PGV value of the seismic records in the Taipei basin were significantly amplified during the 1999 Chi-Chi mainshock. In this study, the extended fault simulation method is applied to the soil stations (NEHRP class D) located in the Taipei basin to see whether the basin effect is significant for PSA as well and at which frequencies the basin effect significantly amplifies the motions. Figure 5.37 shows a topographic map of the Taipei metropolitan area. In this study, about 24 soil stations (NEHRP class D) located in Taipei basin are used (up-triangle in Figure 5.37). According to the geological province map of Taiwan (see Figure 2.13), Taipei basin is located in Holocene Alluvium which is one of the softest layers in Taiwan. The procedure of the simulation for the soil stations in Taipei basin is the same as that for nonlinearity 
correction, that is, the ground motion at these soil stations was simulated using the reference site amplification function (NEHRP class C) and then the linear, nonlinear corrections are applied just to stations located in the Taipei basin. Figure 5.38 is the model bias of the simulation indicating a strong basin amplification between $0.4 \mathrm{~Hz}$ and $2 \mathrm{~Hz}$, especially around $1 \mathrm{~Hz}$, for high frequencies the effect is not significant. Figure 5.40, Figure 5.41 and Figure 5.42 are the model bias versus distance for 0.5 $\mathrm{Hz}, 1 \mathrm{~Hz}$ and $10 \mathrm{~Hz}$ respectively. These three figures clearly demonstrate the basin effect of the soil stations in the Taipei basin, for low to intermediate frequencies.

If I subtract the bias given by Figure 5.32 from bias given by Figure 5.38, the basin effect is separated out from the nonlinear effect, on average. Thus, the basin effect, by itself, is given in Figure 5.39. 
Table 5.3: Linear and nonlinear correction coefficients used in this study. After Choi and Stewart (2005).

\begin{tabular}{|l|c|c|c|}
\hline Period (sec) & $c$ & $b_{1}$ & $b_{2}$ \\
\hline \hline 0.01 & $-0.36 \pm 0.06$ & -0.64 & $-0.14 \pm 0.04$ \\
\hline 0.02 & $-0.34 \pm 0.06$ & -0.63 & $-0.12 \pm 0.04$ \\
\hline 0.03 & $-0.33 \pm 0.06$ & -0.62 & $-0.11 \pm 0.04$ \\
\hline 0.04 & $-0.31 \pm 0.06$ & -0.61 & $-0.11 \pm 0.04$ \\
\hline 0.05 & $-0.29 \pm 0.06$ & -0.64 & $-0.11 \pm 0.04$ \\
\hline 0.06 & $-0.25 \pm 0.06$ & -0.64 & $-0.11 \pm 0.04$ \\
\hline 0.075 & $-0.23 \pm 0.06$ & -0.64 & $-0.11 \pm 0.04$ \\
\hline 0.09 & $-0.23 \pm 0.07$ & -0.64 & $-0.12 \pm 0.04$ \\
\hline 0.1 & $-0.25 \pm 0.07$ & -0.6 & $-0.13 \pm 0.04$ \\
\hline 0.12 & $-0.26 \pm 0.07$ & -0.56 & $-0.14 \pm 0.04$ \\
\hline 0.15 & $-0.28 \pm 0.07$ & -0.53 & $-0.18 \pm 0.04$ \\
\hline 0.17 & $-0.29 \pm 0.07$ & -0.53 & $-0.19 \pm 0.04$ \\
\hline 0.2 & $-0.31 \pm 0.07$ & -0.52 & $-0.19 \pm 0.04$ \\
\hline 0.24 & $-0.38 \pm 0.07$ & -0.52 & $-0.16 \pm 0.04$ \\
\hline 0.3 & $-0.44 \pm 0.07$ & -0.52 & $-0.14 \pm 0.05$ \\
\hline 0.36 & $-0.48 \pm 0.07$ & -0.51 & $-0.11 \pm 0.05$ \\
\hline 0.4 & $-0.5 \pm 0.07$ & -0.51 & $-0.1 \pm 0.05$ \\
\hline 0.46 & $-0.55 \pm 0.07$ & -0.5 & $-0.08 \pm 0.05$ \\
\hline 0.5 & $-0.6 \pm 0.07$ & -0.5 & $-0.06 \pm 0.05$ \\
\hline 0.6 & $-0.66 \pm 0.07$ & -0.49 & $-0.03 \pm 0.05$ \\
\hline 0.75 & $-0.69 \pm 0.07$ & -0.47 & $0 \pm 0.05$ \\
\hline 0.85 & $-0.69 \pm 0.07$ & -0.46 & $0 \pm 0.05$ \\
\hline 1 & $-0.7 \pm 0.07$ & -0.44 & $0 \pm 0.05$ \\
\hline 1.5 & $-0.72 \pm 0.08$ & -0.4 & $0 \pm 0.06$ \\
\hline 2 & $-0.73 \pm 0.08$ & -0.38 & $0 \pm 0.06$ \\
\hline 3 & $-0.74 \pm 0.09$ & -0.34 & $0 \pm 0.07$ \\
\hline 4 & $-0.75 \pm 0.09$ & -0.31 & $0 \pm 0.07$ \\
\hline 5 & $-0.75 \pm 0.14$ & -0.3 & $0 \pm 0.11$ \\
\hline & & & \\
\hline 0.5 & & & \\
\hline & & -19 &
\end{tabular}




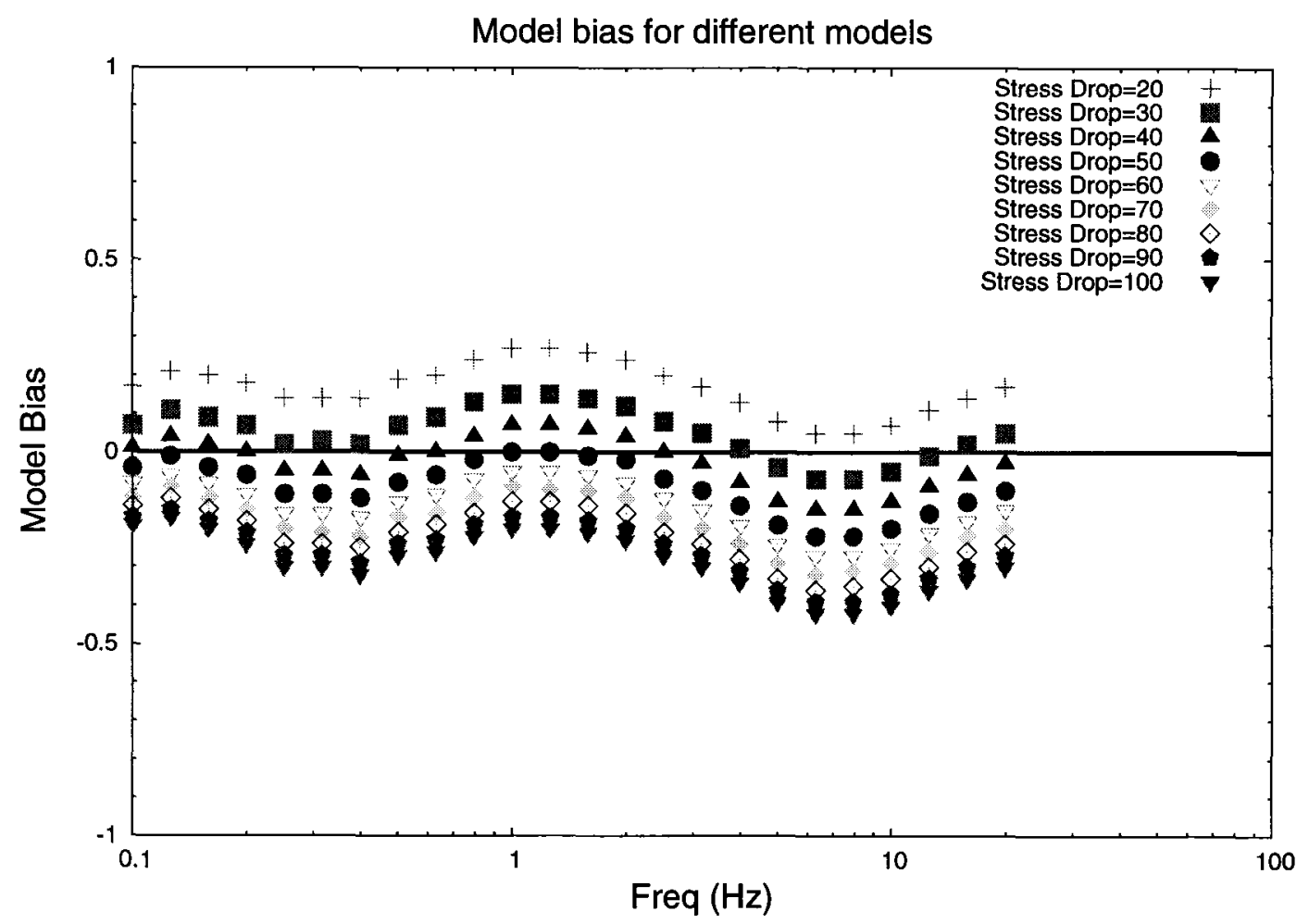

Figure 5.1. Model bias comparison for models with different stress drop (the stress drop increases from 20 bars to 100 bars with step 10 bars) but fixed pulsing percentage (50\%) 


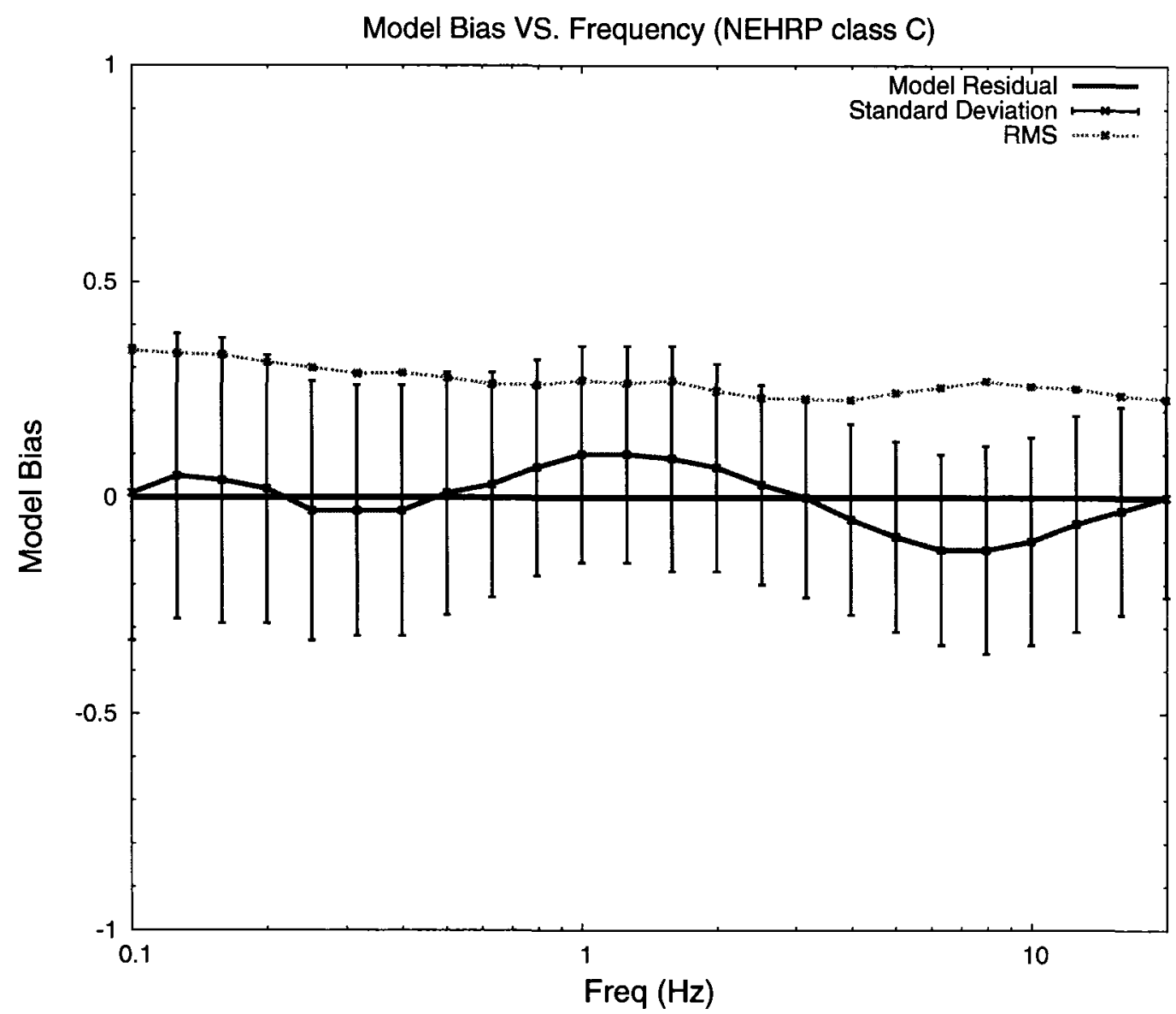

Figure 5.2. Model bias for the preferred model on rock stations (NEHRP class C) with stress drop $=40$ bars and pulsing percentage $=80 \%$; RMS is the root mean square of the model bias 


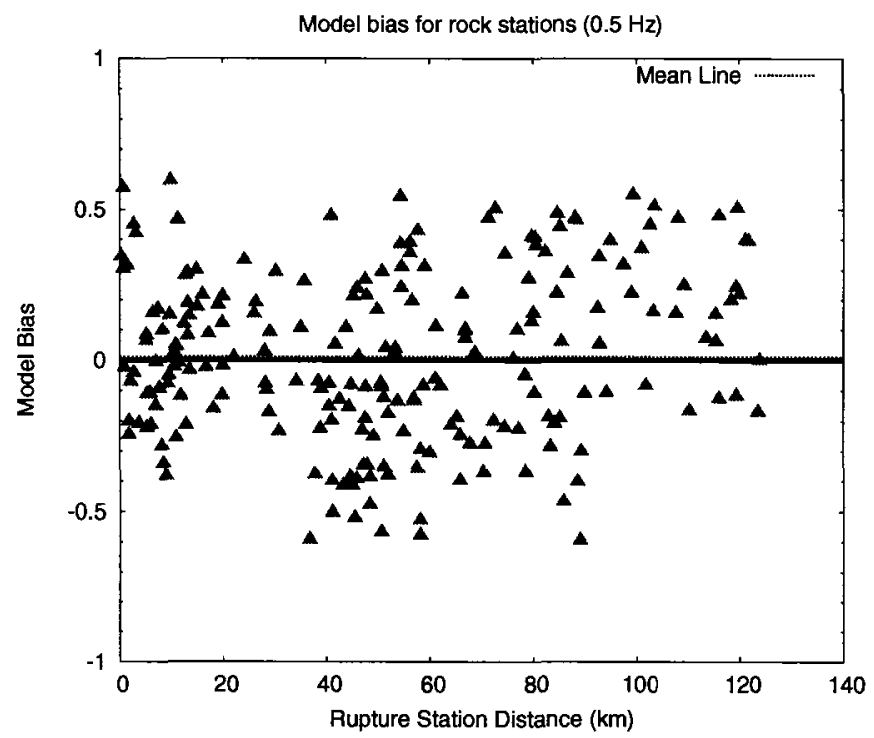

Figure 5.3. Model bias versus distance for the preferred model (NEHRP class C) at $0.5 \mathrm{~Hz}$ (stress drop $=40$ bars, pulsing percentage $=80 \%$ )

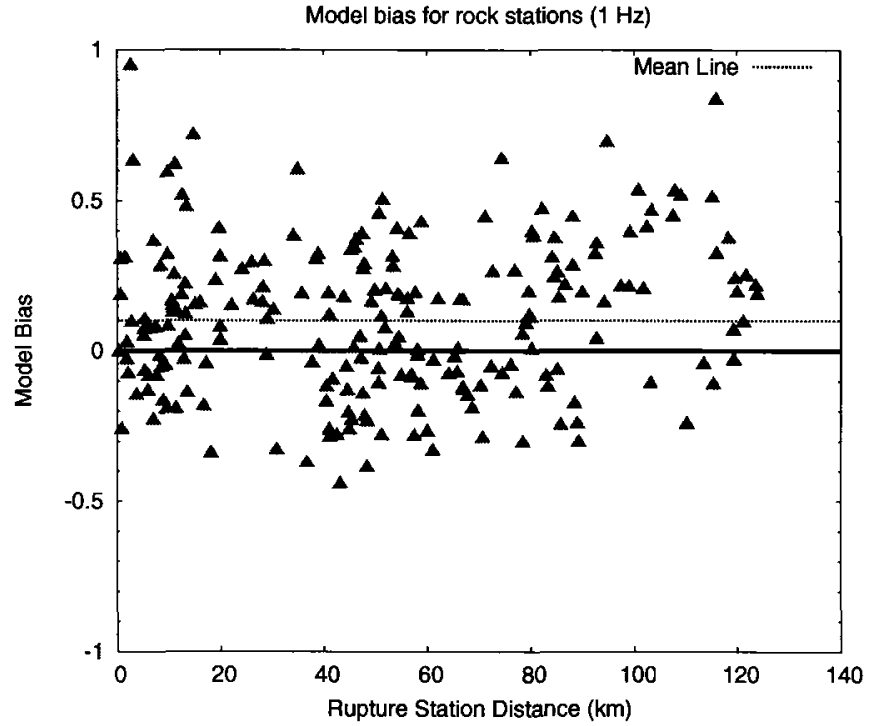

Figure 5.4. Model bias versus distance for the preferred model (NEHRP class C) at $1 \mathrm{~Hz}$ (stress drop $=40$ bars, pulsing percentage $=80 \%$ ) 


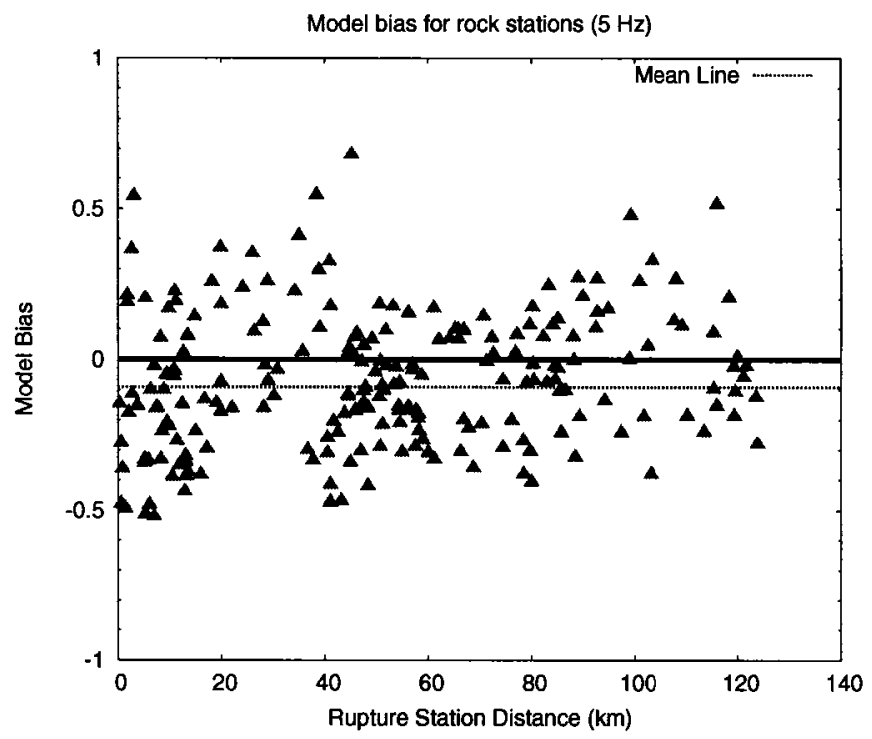

Figure 5.5. Model bias versus distance for the preferred model (NEHRP class C) at $5 \mathrm{~Hz}$ (stress drop $=40$ bars, pulsing percentage $=80 \%$ )

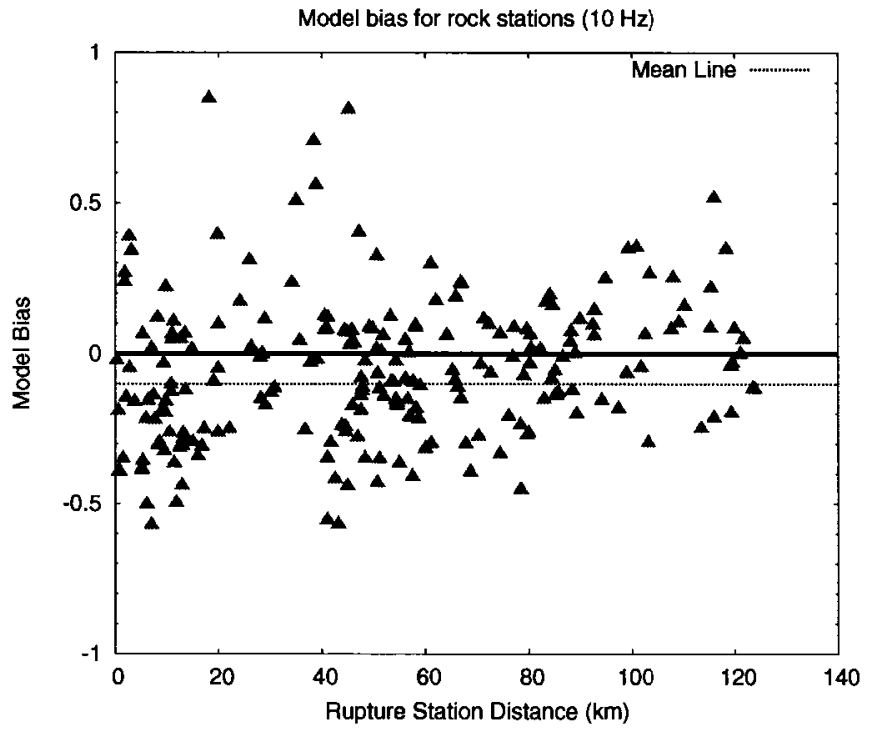

Figure 5.6. Model bias versus distance for the preferred model (NEHRP class C) at $10 \mathrm{~Hz}$ (stress drop $=40$ bars, pulsing percentage $=80 \%$ ) 


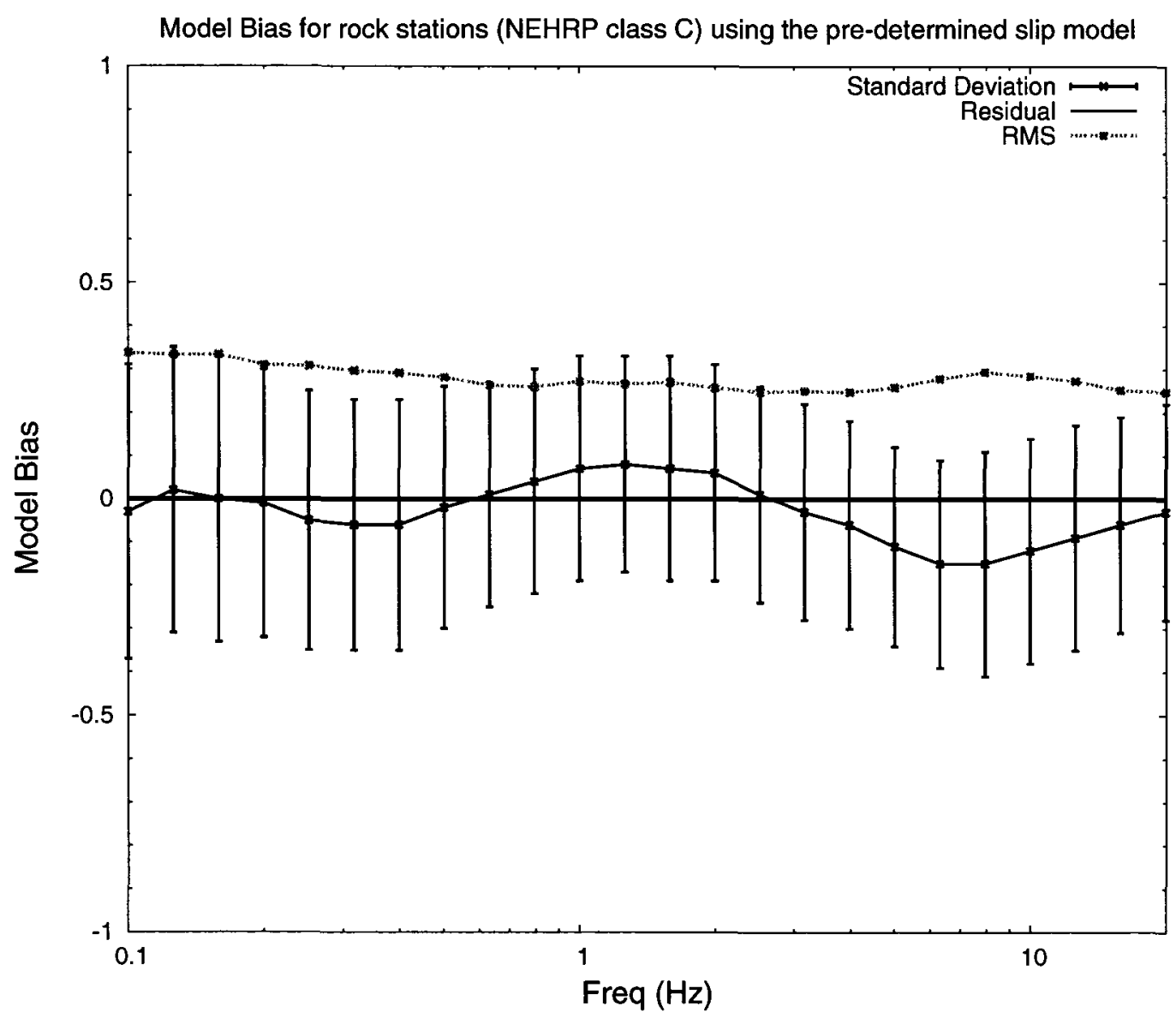

Figure 5.7. Model bias for the preferred model on rock stations (NEHRP class C) using the pre-determined slip model (stress drop $=40$ bars, pulsing percentage $=$ $80 \%$ ); RMS is the root mean square of the model bias 


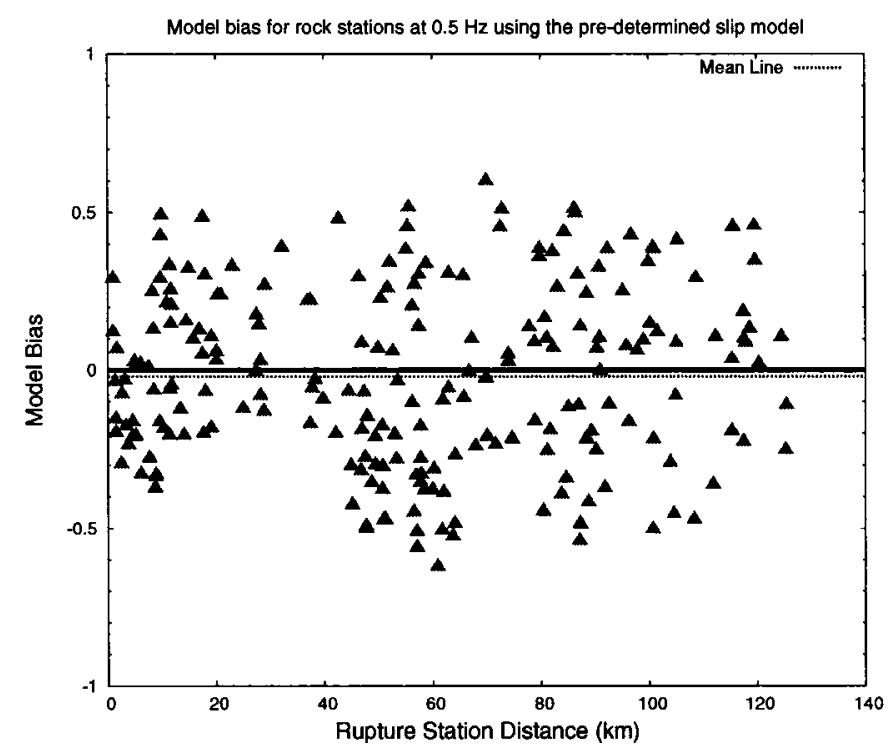

Figure 5.8. Model bias versus distance for the pre-determined slip model (NEHRP class $\mathrm{C}$ ) at $0.5 \mathrm{~Hz}$ (stress drop $=40$ bars, pulsing percentage $=80 \%$ )

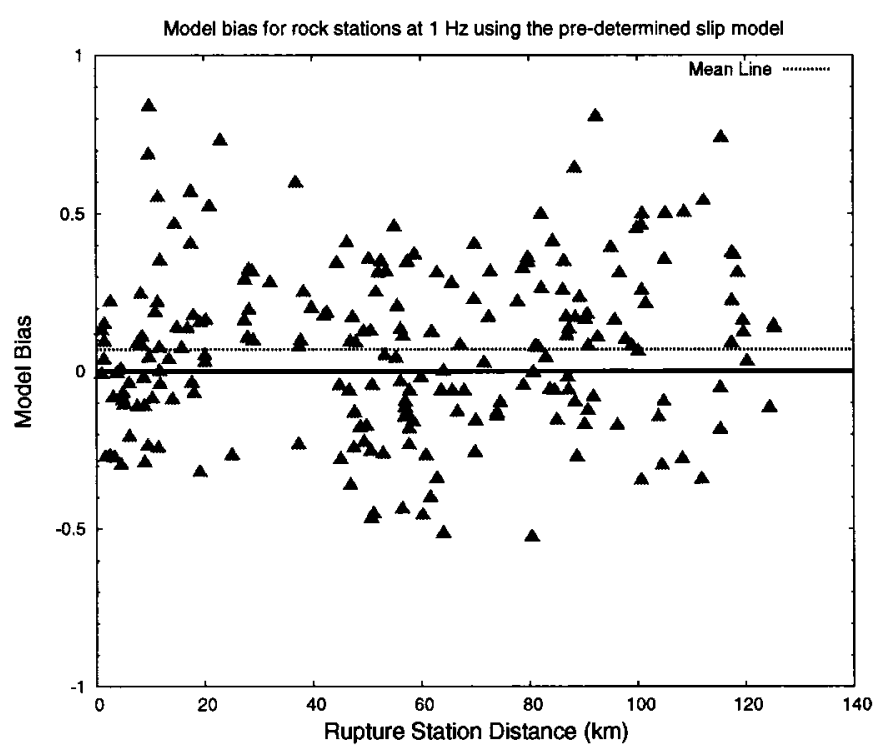

Figure 5.9. Model bias versus distance for pre-determined slip model (NEHRP class C) at $1 \mathrm{~Hz}$ (stress drop $=40$ bars, pulsing percentage $=80 \%$ ) 


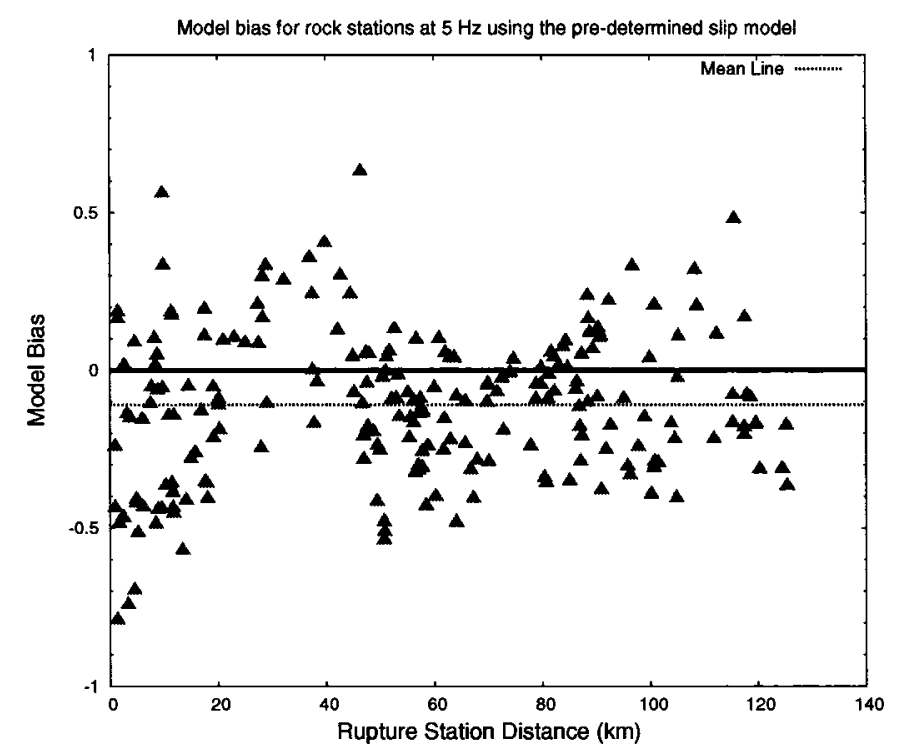

Figure 5.10. Model bias versus distance for pre-determined slip model (NEHRP class C) at $5 \mathrm{~Hz}$ (stress drop $=40$ bars, pulsing percentage $=80 \%$ )

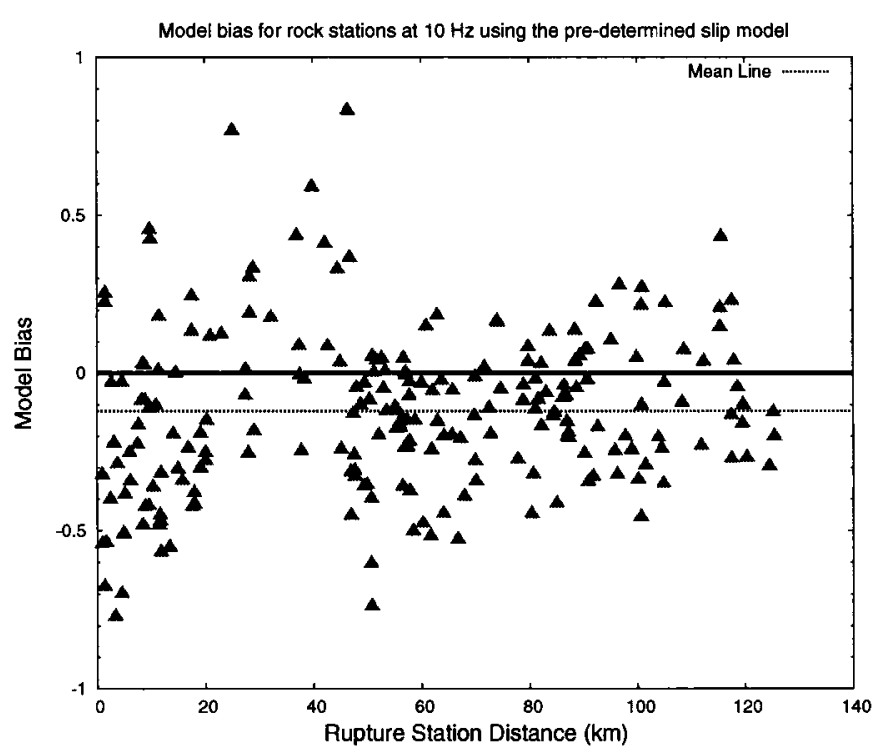

Figure 5.11. Model bias versus distance for pre-determined slip model (NEHRP class C) at $10 \mathrm{~Hz}$ (stress drop $=40$ bars, pulsing percentage $=80 \%$ ) 

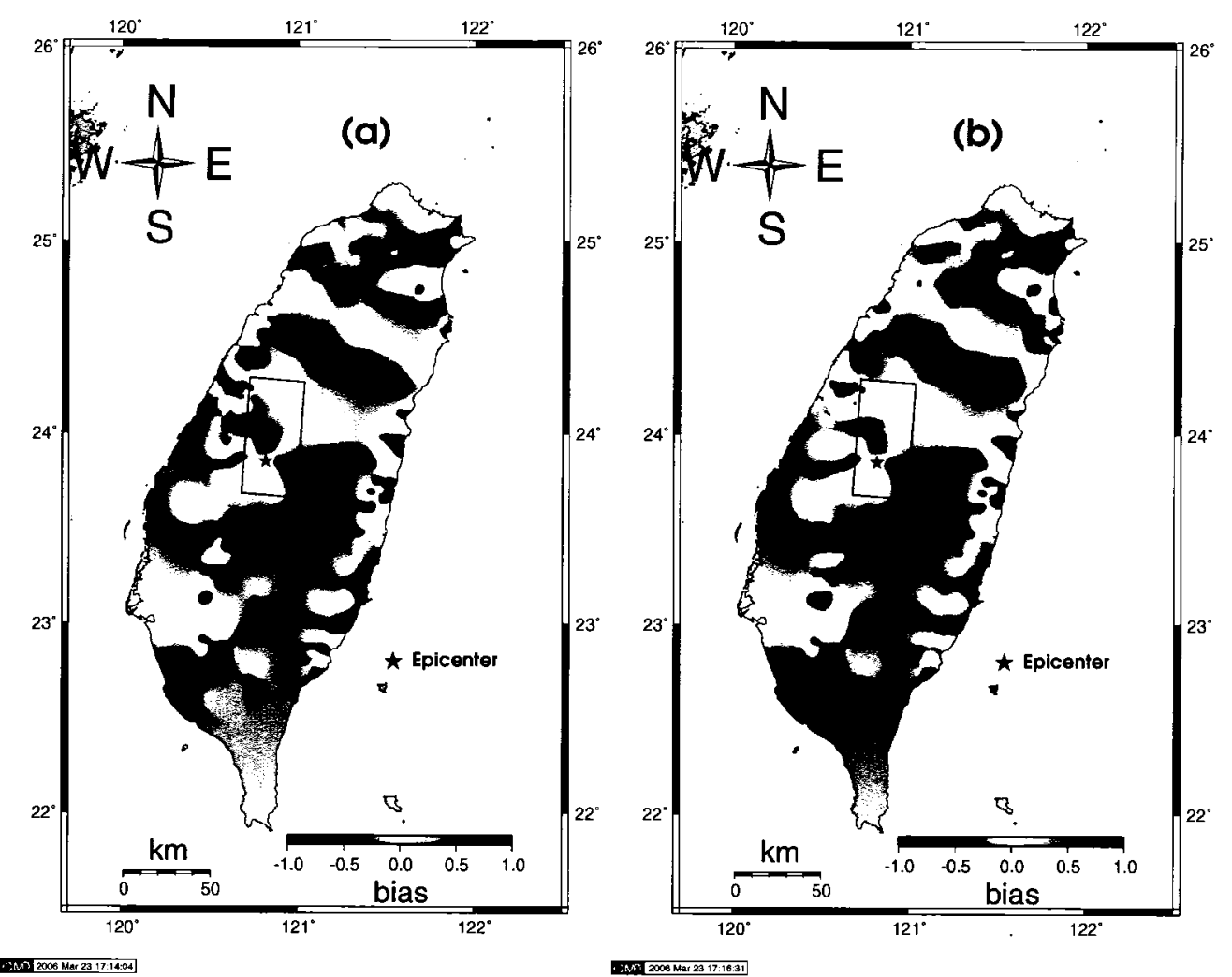

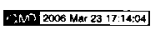

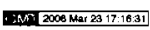

Figure 5.12. (a) Map view of the model bias for the preferred model (NEHRP class C) using the random slip distribution model $(1 \mathrm{~Hz})$ (b) Map view of the model bias for the preferred model (NEHRP class C) using the pre-determined slip distribution model $(1 \mathrm{~Hz})$; the rectangle is the fault plane (Wang et al., 2001) 

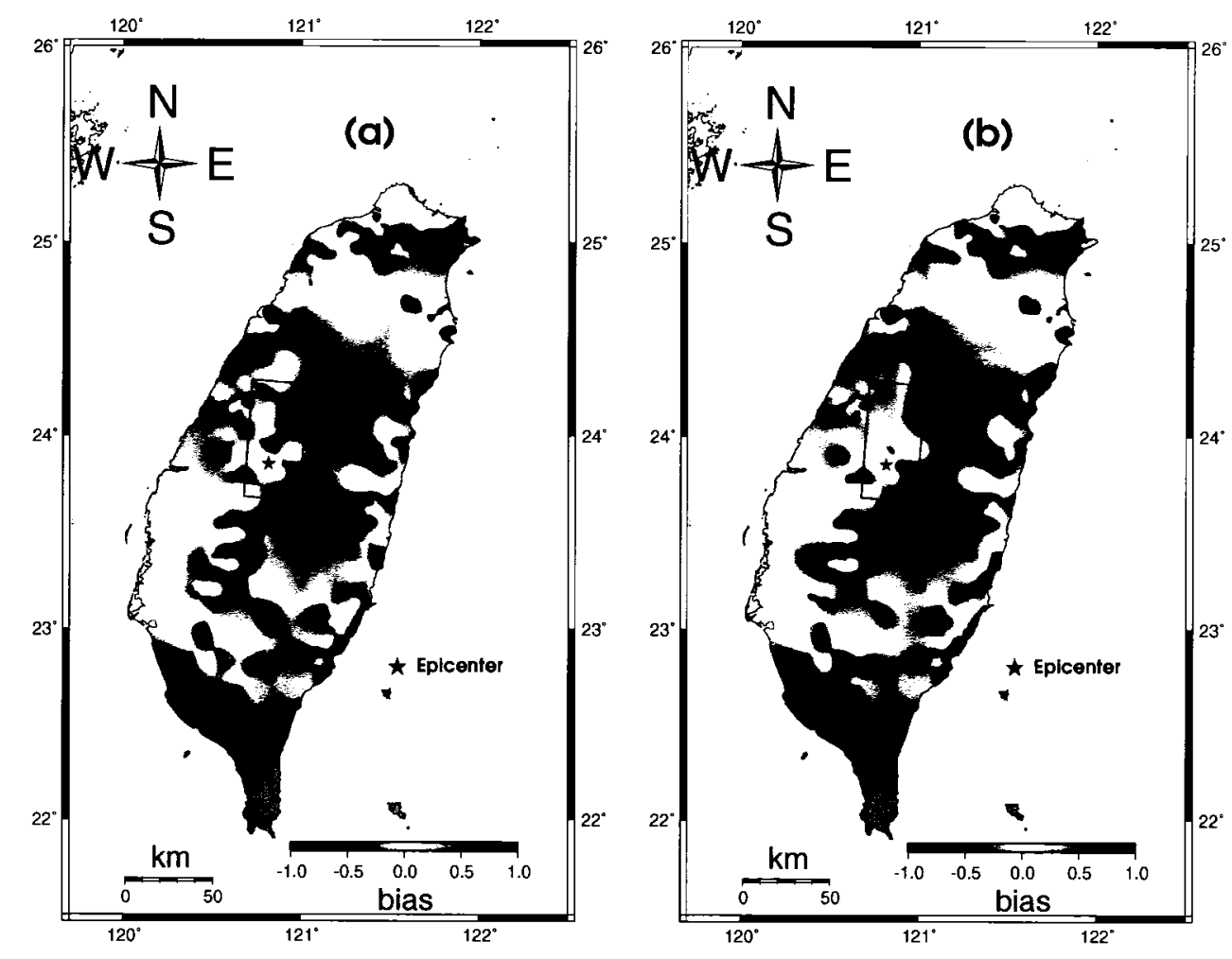

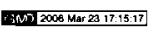

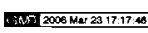

Figure 5.13. (a) Map view of the model bias for the preferred model (NEHRP class C) using the random slip distribution model $(10 \mathrm{~Hz})$ (b) Map view of the model bias for the preferred model (NEHRP class C) using the pre-determined slip distribution model $(10 \mathrm{~Hz})$; the rectangle is the fault plane (Wang et al., 2001) 


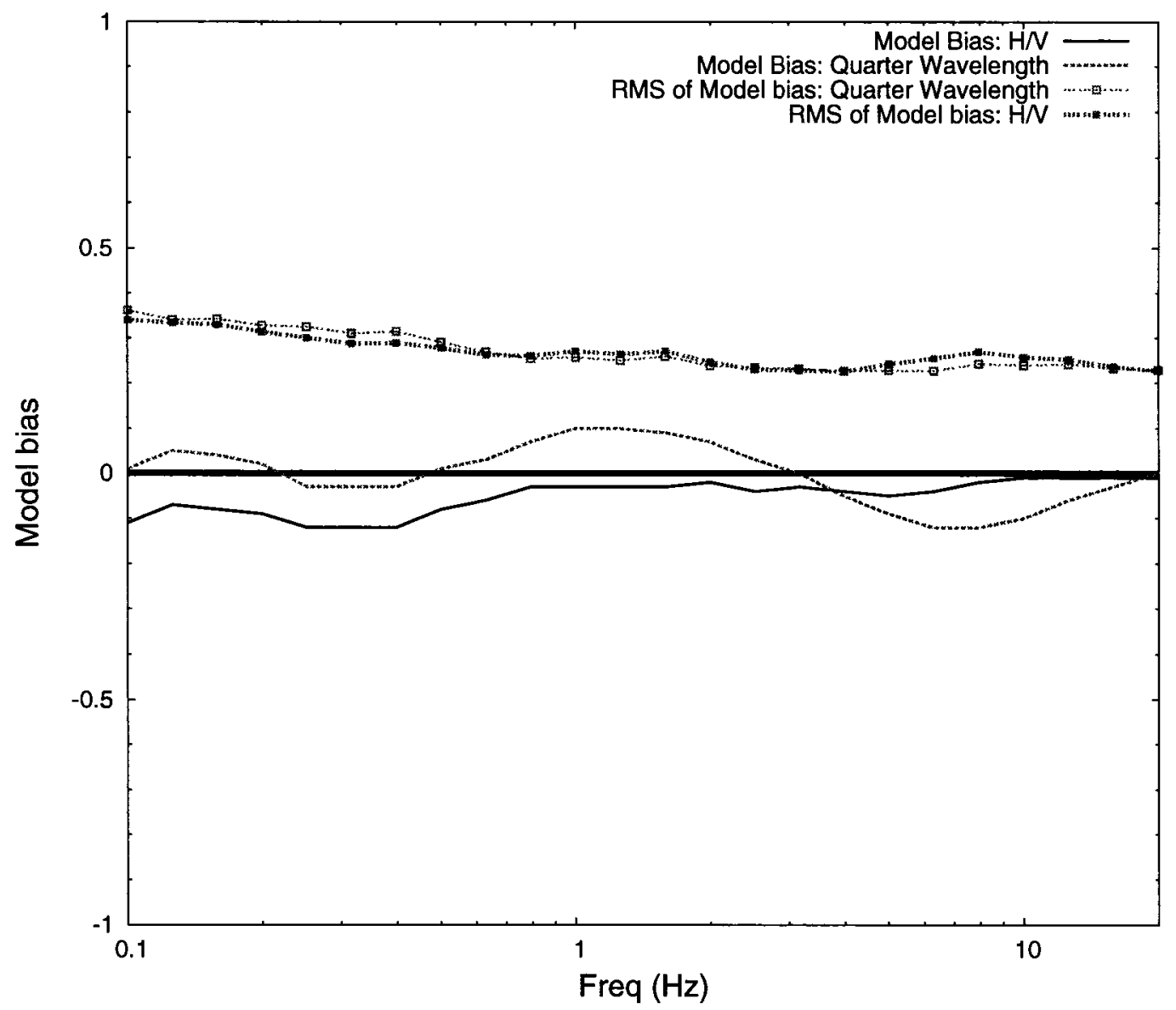

Figure 5.14. Comparison of the model bias for the models with $\mathrm{H} / \mathrm{V}$ ratio as site amplification and with quarter wavelength approximation as site amplification (stress drop $=40$ bars, pulsing percentage $=80 \%$ ); RMS is the root mean square of the model bias 


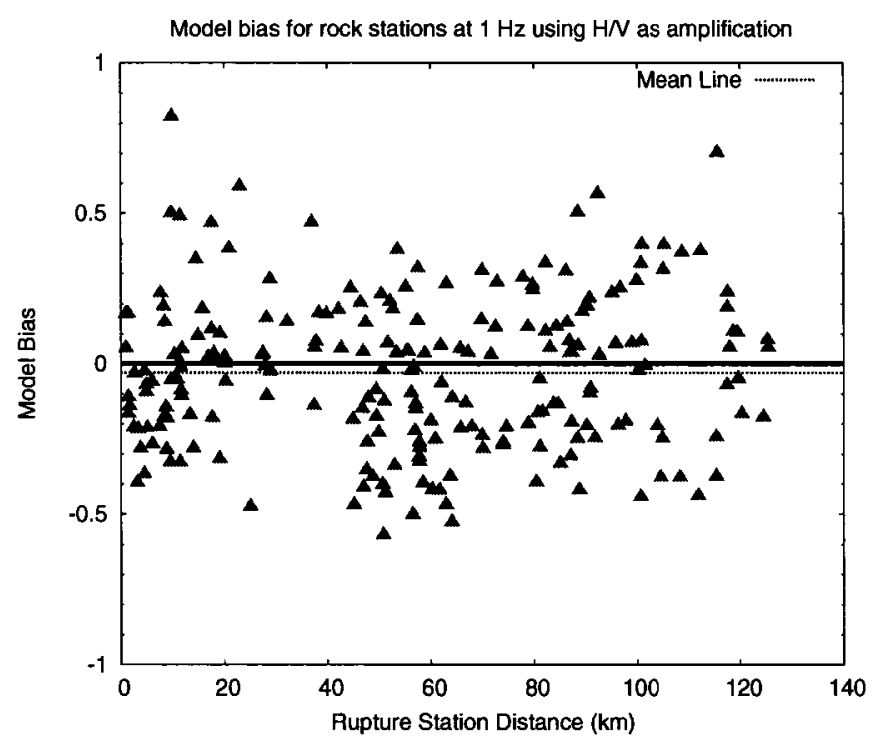

Figure 5.15. Model bias versus distance for the preferred model (NEHRP class C) using $\mathrm{H} / \mathrm{V}$ as site amplification at $1 \mathrm{~Hz}$ (stress drop $=40$ bars, pulsing percentage $=$ $80 \%)$

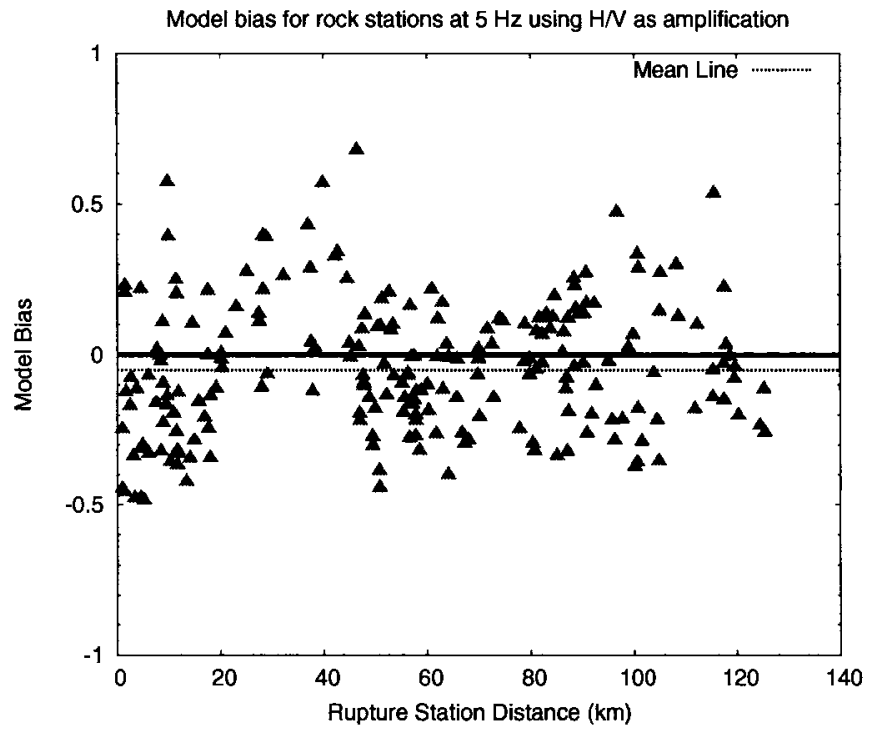

Figure 5.16. Model bias versus distance for the preferred model (NEHRP class C) using $\mathrm{H} / \mathrm{V}$ as site amplification at $5 \mathrm{~Hz}$ (stress drop $=40$ bars, pulsing percentage $=$ $80 \%$ ) 


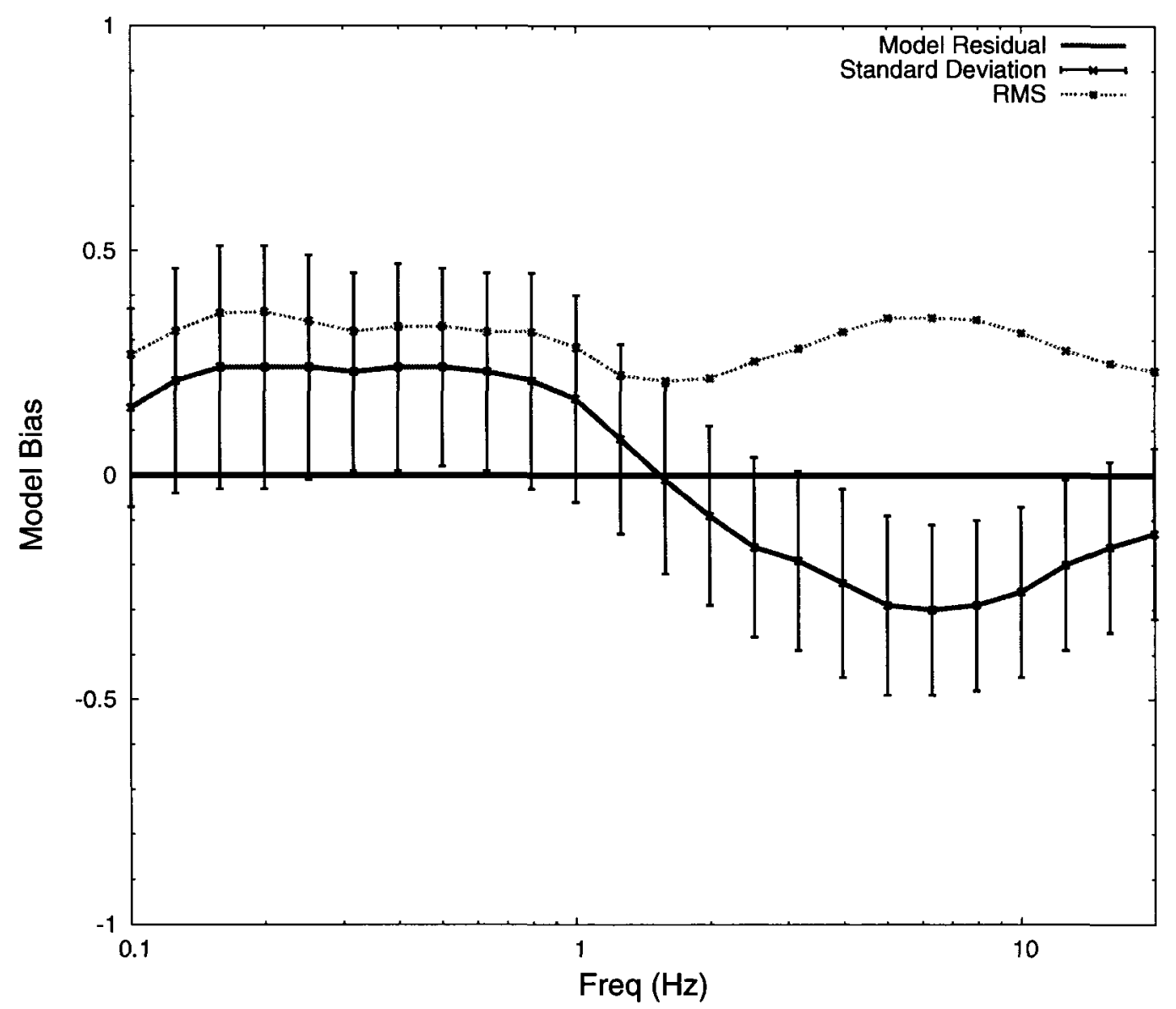

Figure 5.17. Model bias versus frequency for soil stations (NEHRP class D sites) using linear site amplification factor calculated by quarter wavelength approximation; RMS is the root mean square of the model bias 


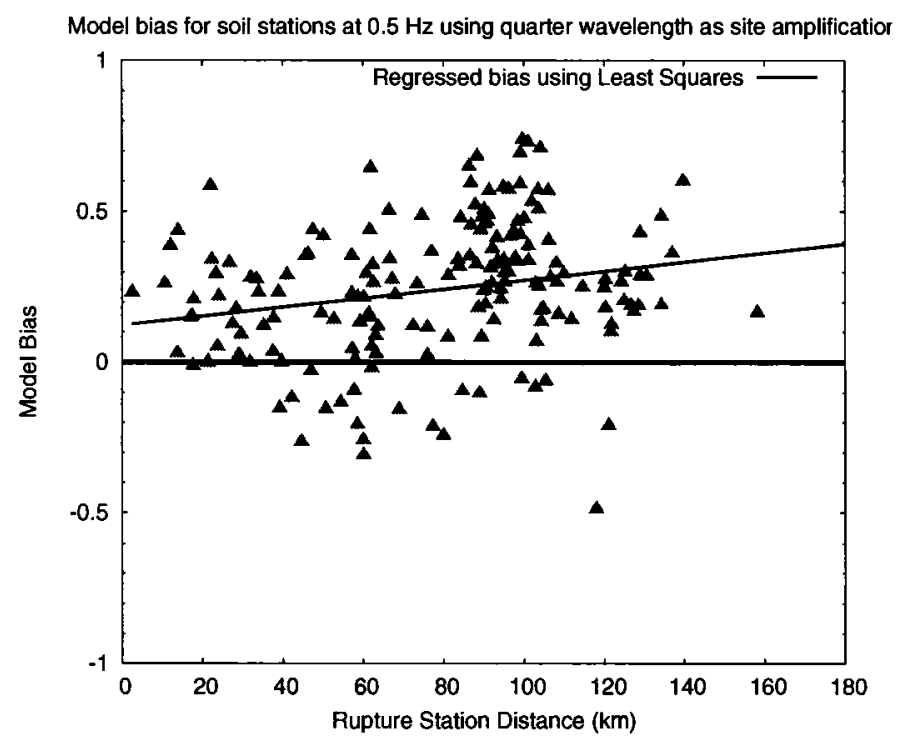

Figure 5.18. Model bias versus distance for soil stations (NEHRP class D sites) using linear site amplification factor calculated by quarter wavelength approximation $(0.5$ $\mathrm{Hz})$

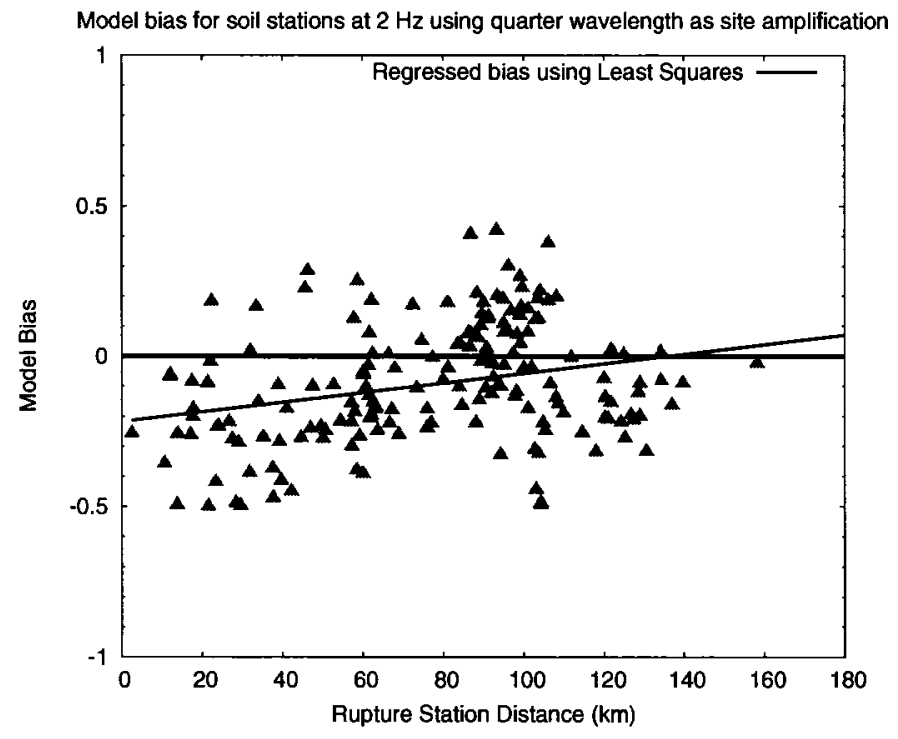

Figure 5.19. Model bias versus distance for soil stations (NEHRP class D sites) using linear site amplification factor calculated by quarter wavelength approximation (2 $\mathrm{Hz}$ ) 


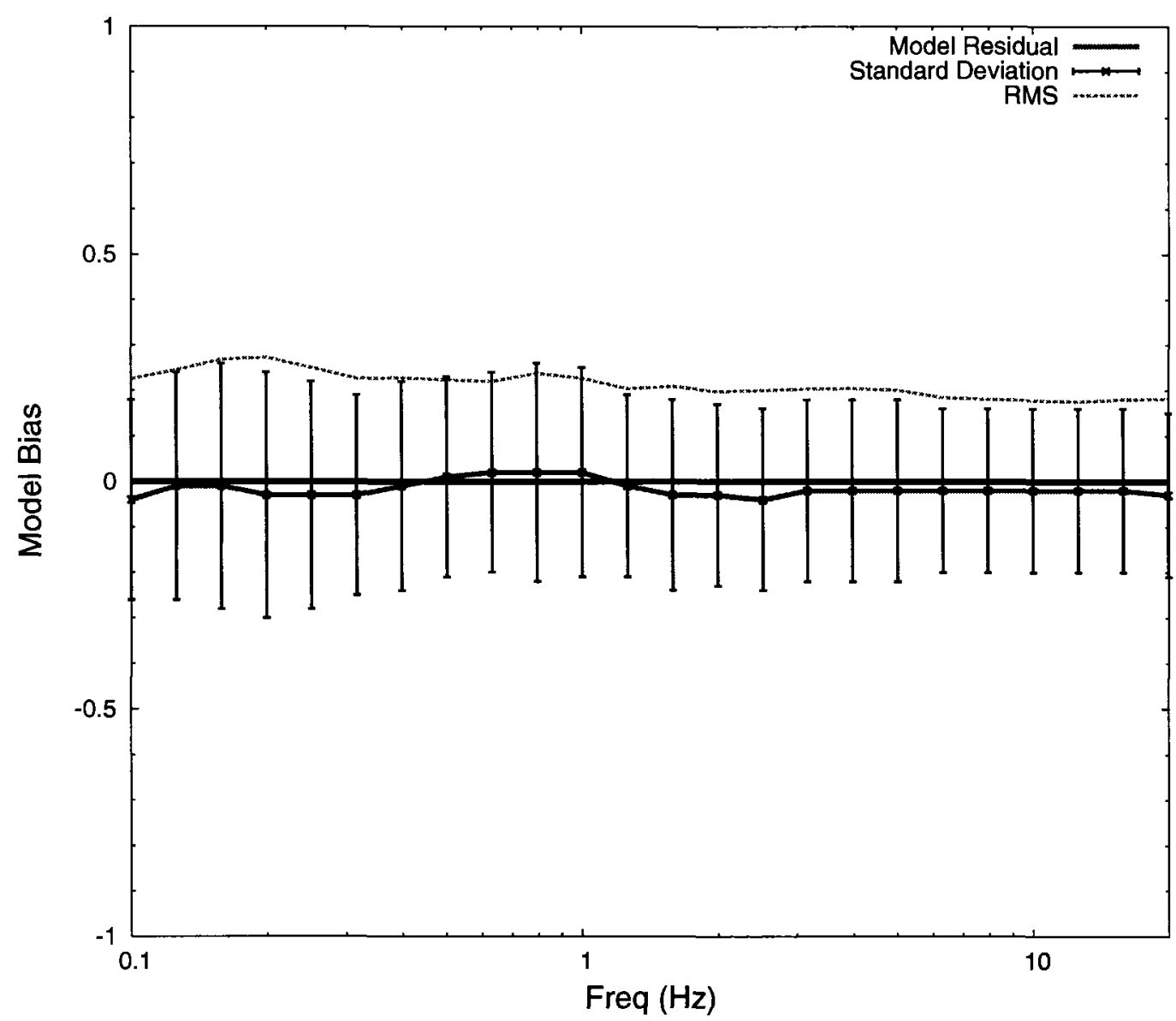

Figure 5.20. Model bias versus frequency for soil stations (NEHRP class D sites) using $\mathrm{H} / \mathrm{V}$ ratio as site amplification factor; RMS is the root mean square of the model bias 


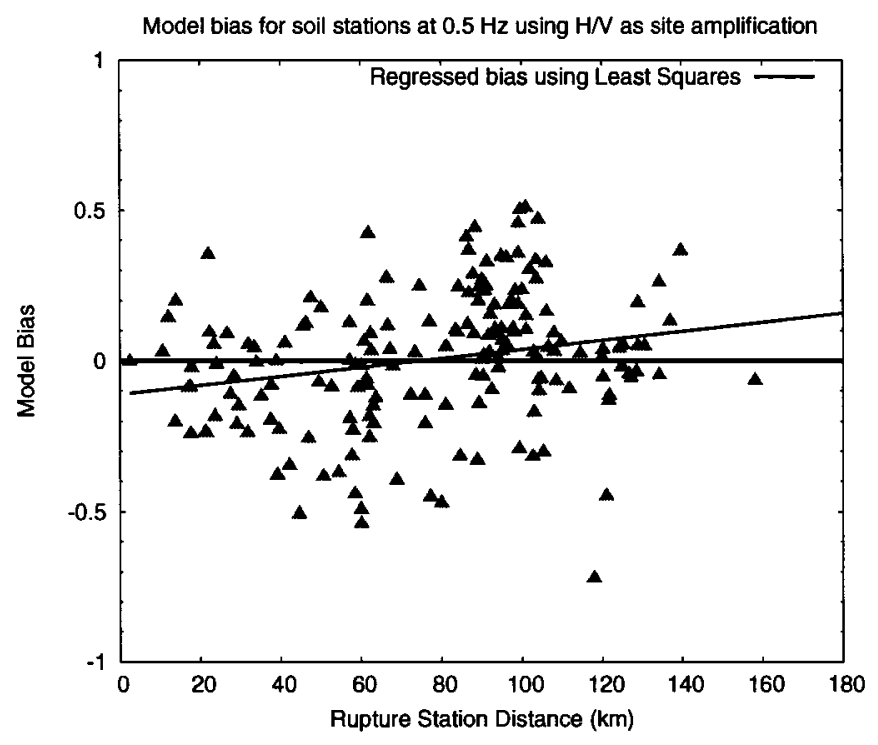

Figure 5.21. Model bias versus distance for soil stations (NEHRP class D sites) using $\mathrm{H} / \mathrm{V}$ ratio as site amplification factor $(0.5 \mathrm{~Hz})$

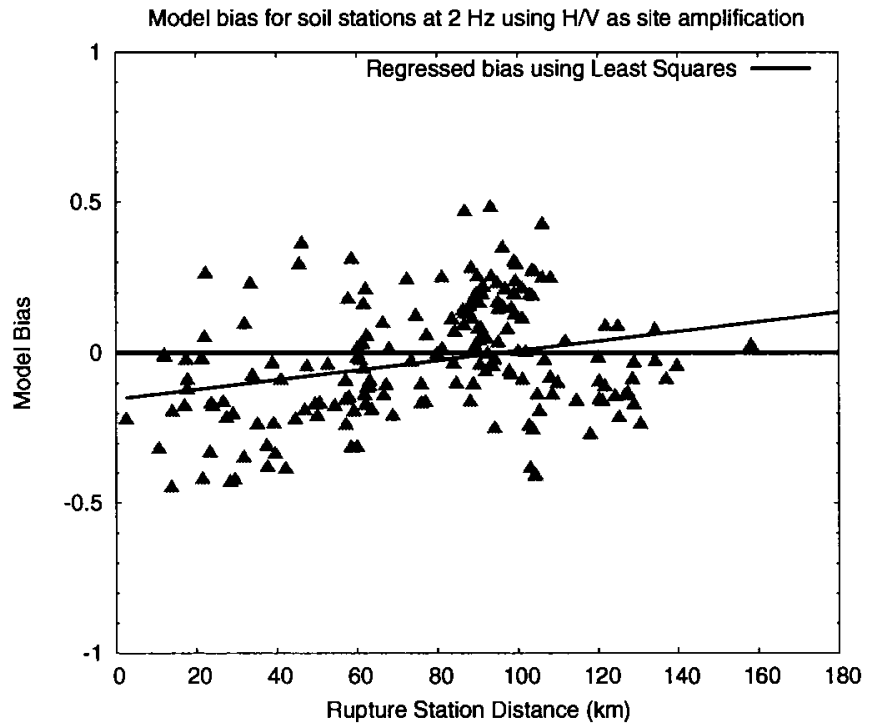

Figure 5.22. Model bias versus distance for soil stations (NEHRP class D sites) using $\mathrm{H} / \mathrm{V}$ ratio as site amplification factor $(2 \mathrm{~Hz})$ 


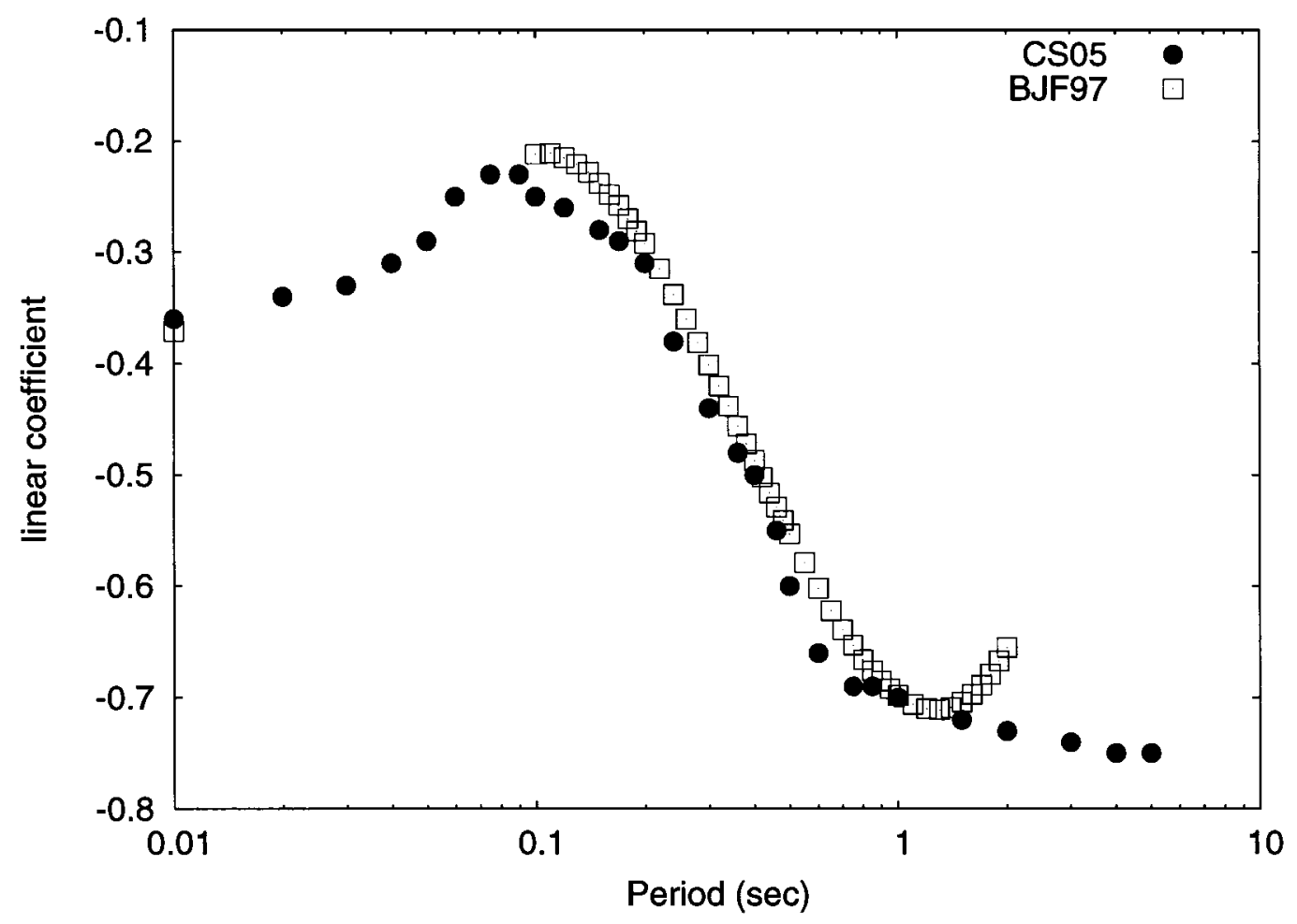

Figure 5.23. Comparison of the linear correction coefficient function between Boore et al. (1997) and Choi and Stewart (2005). 


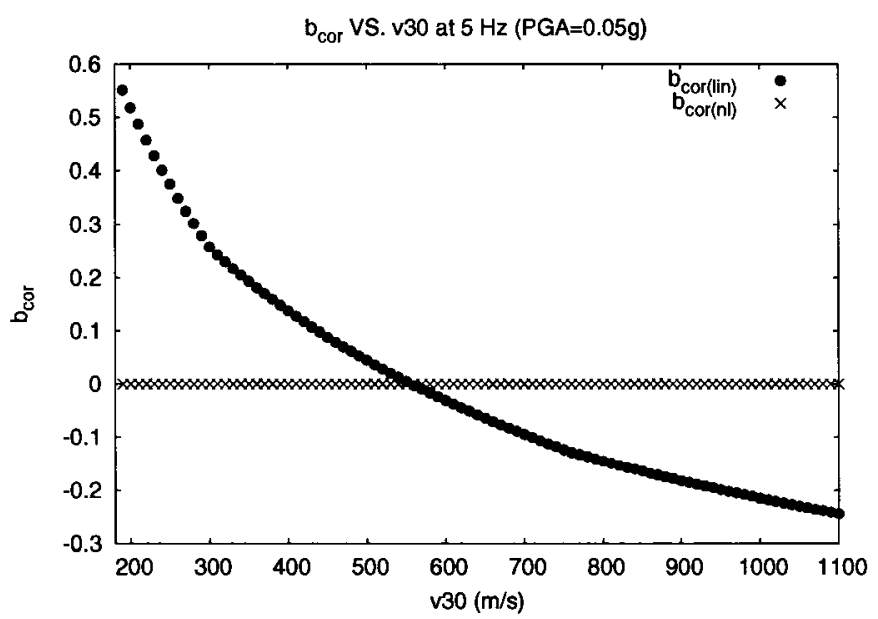

Figure 5.24. Comparison of linear and nonlinear correction term for $\mathrm{PGA}=0.05 \mathrm{~g}$ based on Boore and Atkinson (2005) (5Hz)

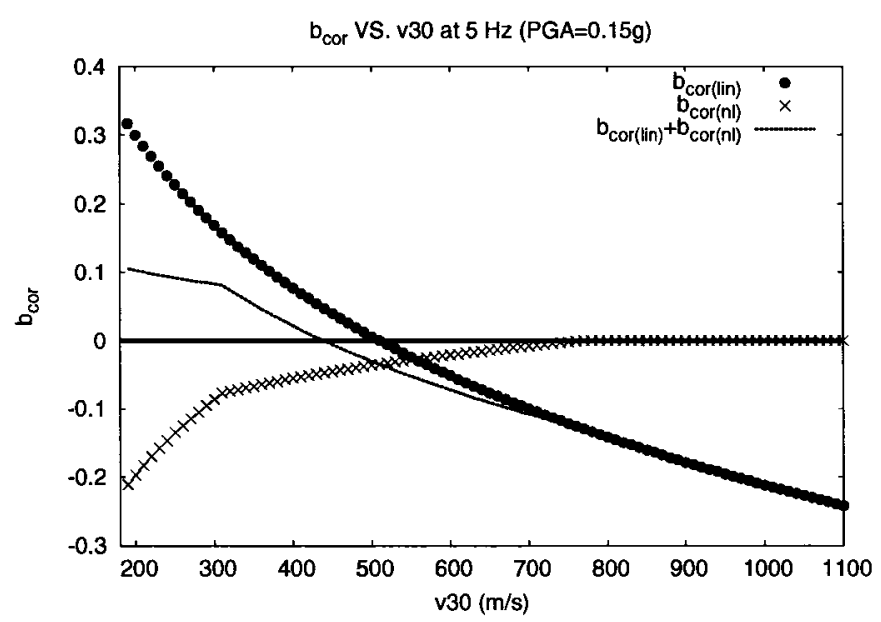

Figure 5.25. Comparison of linear and nonlinear correction term for $P G A=0.15 \mathrm{~g}$ based on Boore and Atkinson (2005) $(5 \mathrm{~Hz})$ 


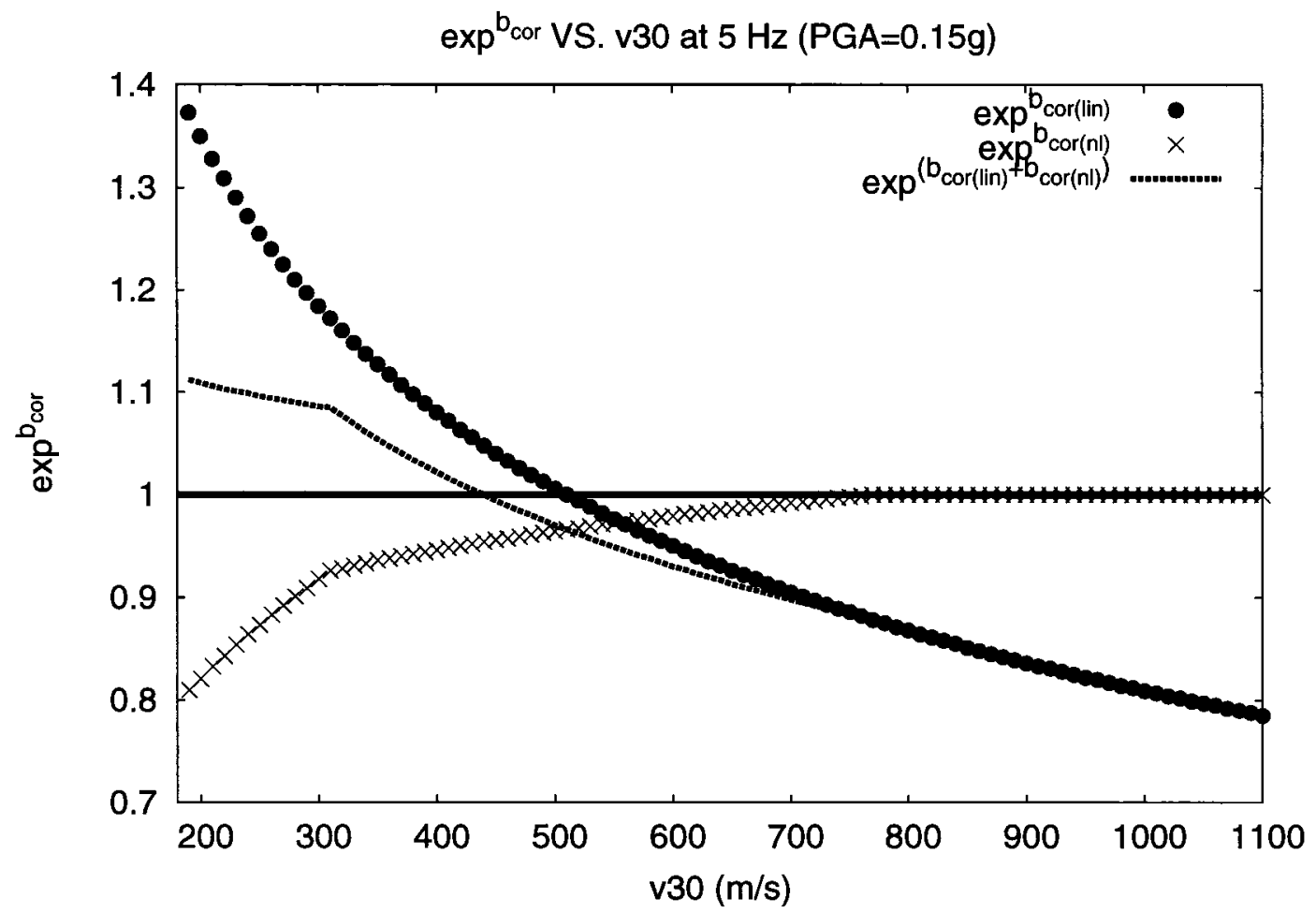

Figure 5.26. Comparison of the exponential form of the linear and nonlinear correction term for PGA $=0.15 \mathrm{~g}$ based on Boore and Atkinson (2005) $(5 \mathrm{~Hz})$ 


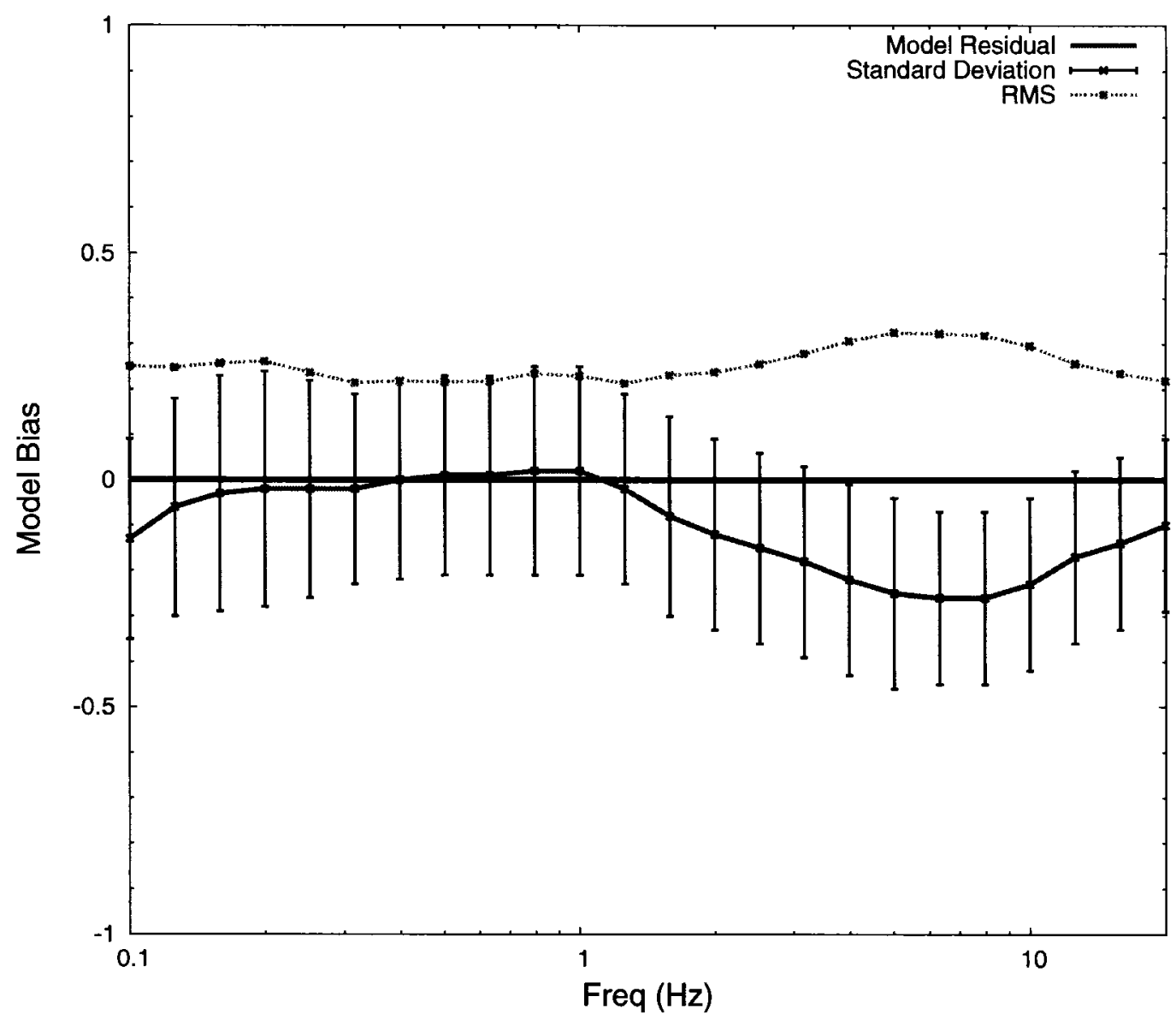

Figure 5.27. Model bias versus frequency for soil stations (NEHRP class D sites) using only linear correction by Boore and Atkinson (2005); RMS is the root mean square of the model bias 


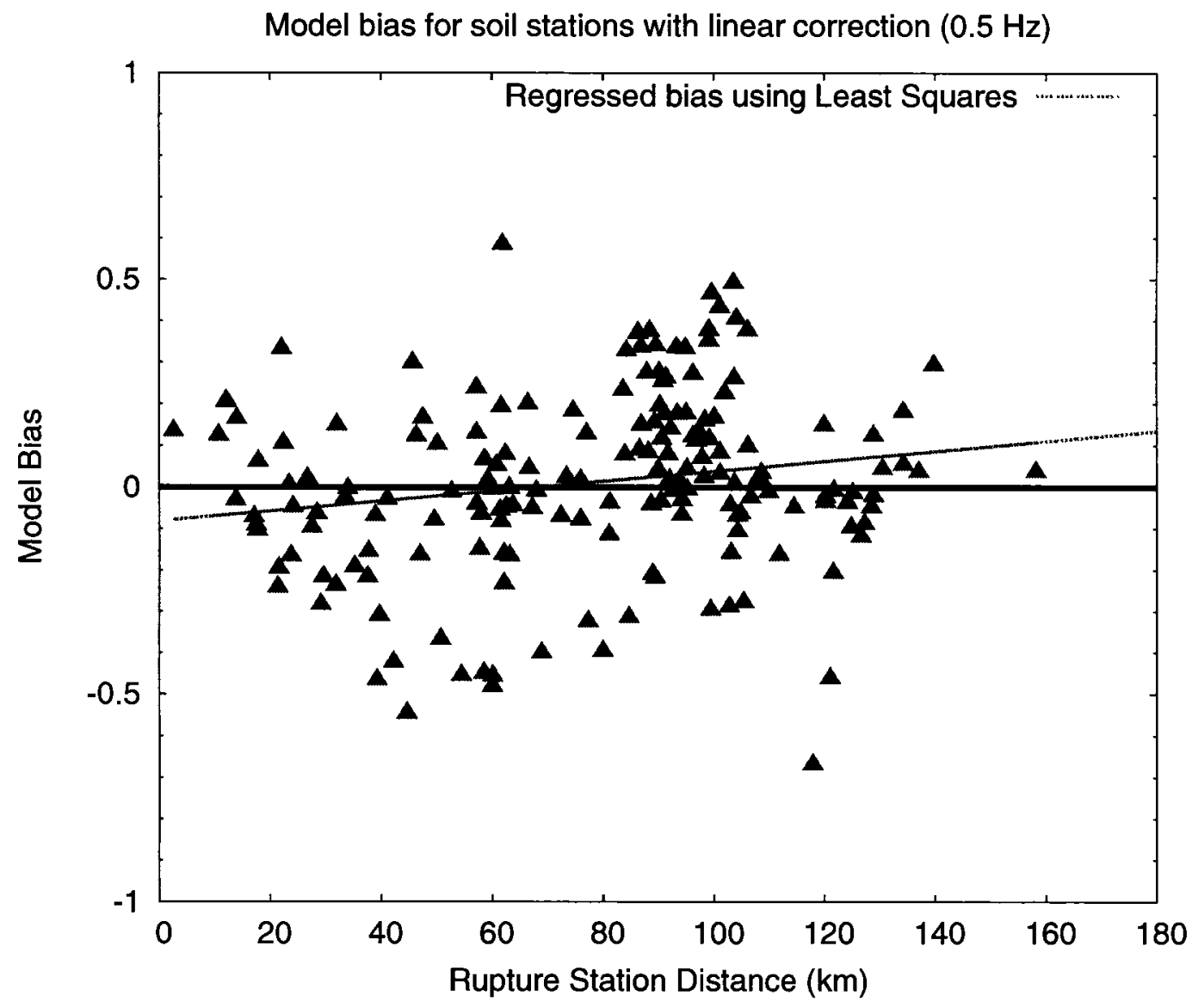

Figure 5.28. Model bias versus distance for soil stations (NEHRP class D sites) after linear correction $(0.5 \mathrm{~Hz})$ using Boore and Atkinson (2005) method 


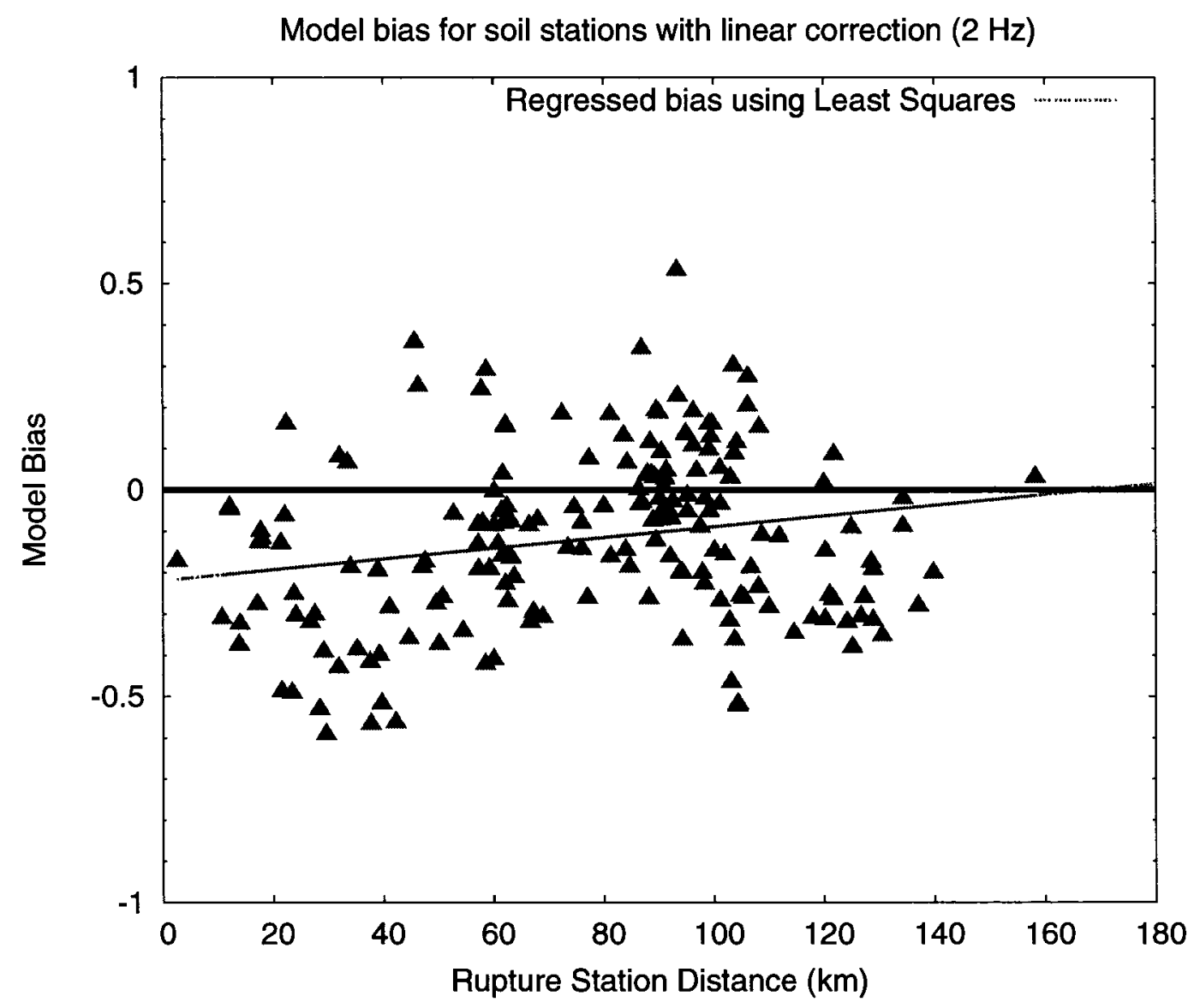

Figure 5.29. Model bias versus distance for soil stations (NEHRP class D sites) after linear correction $(2 \mathrm{~Hz}$ ) using Boore and Atkinson (2005) method 


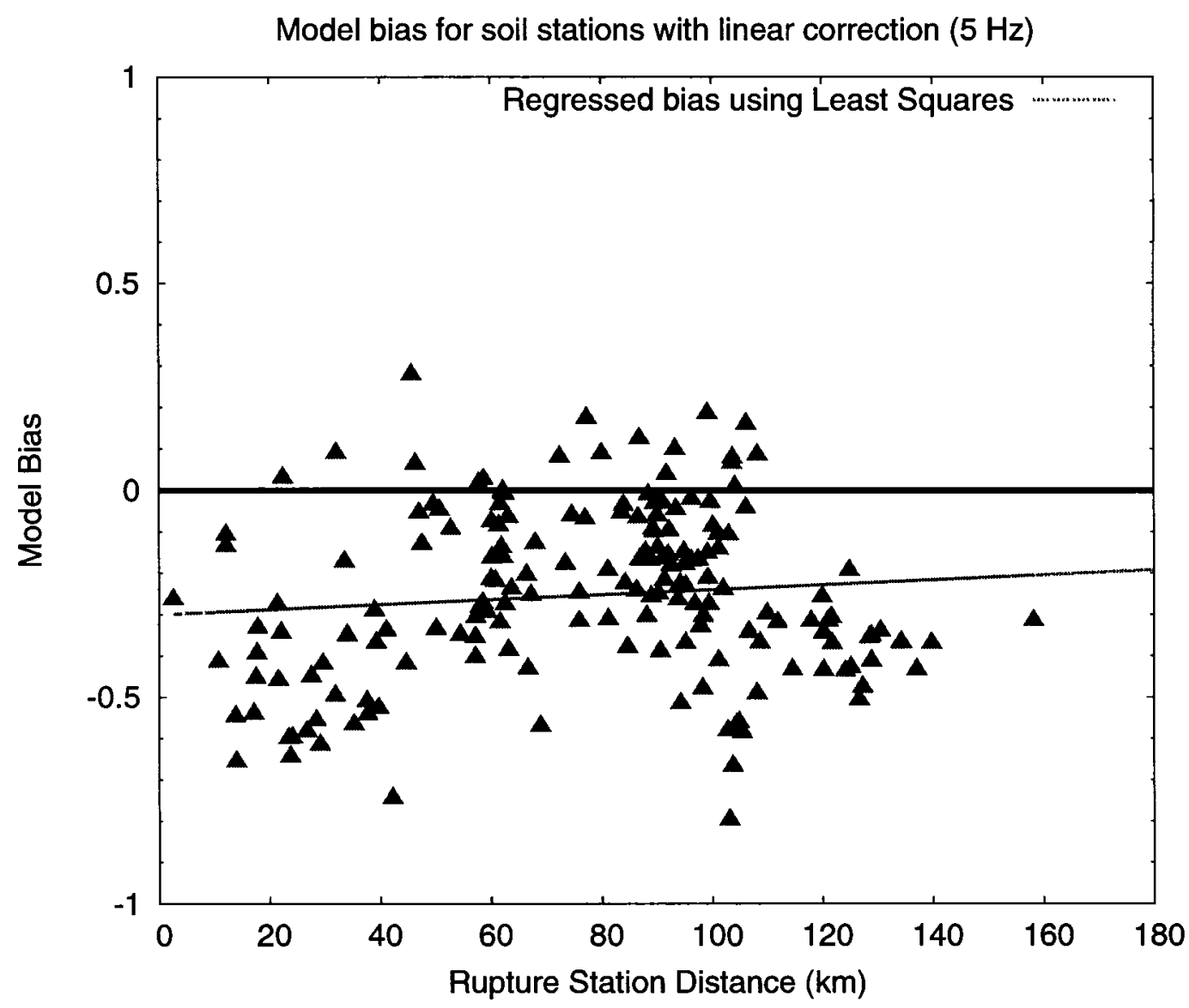

Figure 5.30. Model bias versus distance for soil stations (NEHRP class D sites) after linear correction $(5 \mathrm{~Hz})$ using Boore and Atkinson (2005) method 


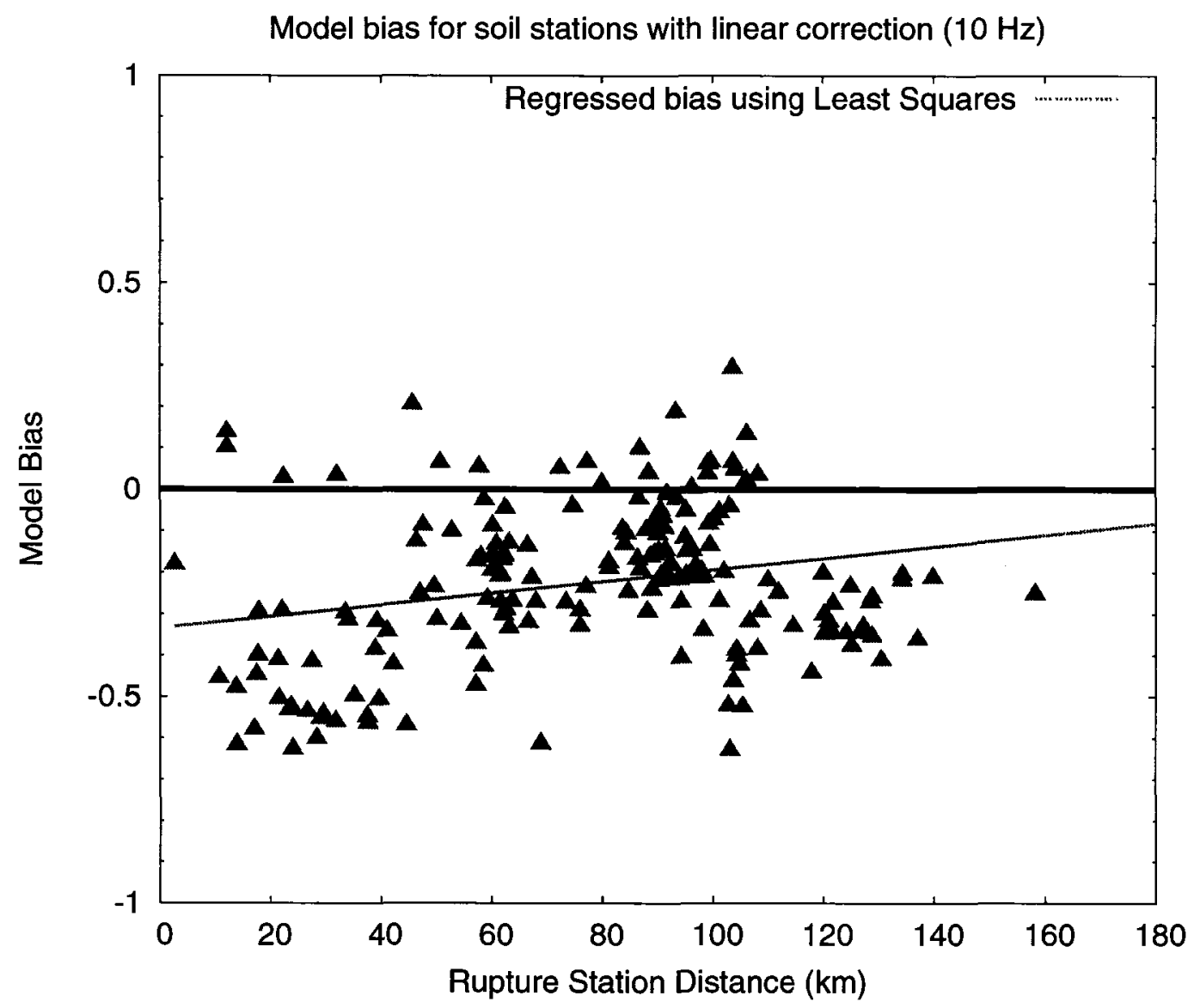

Figure 5.31. Model bias versus distance for soil stations (NEHRP class D sites) after linear correction $(10 \mathrm{~Hz})$ using Boore and Atkinson (2005) method 


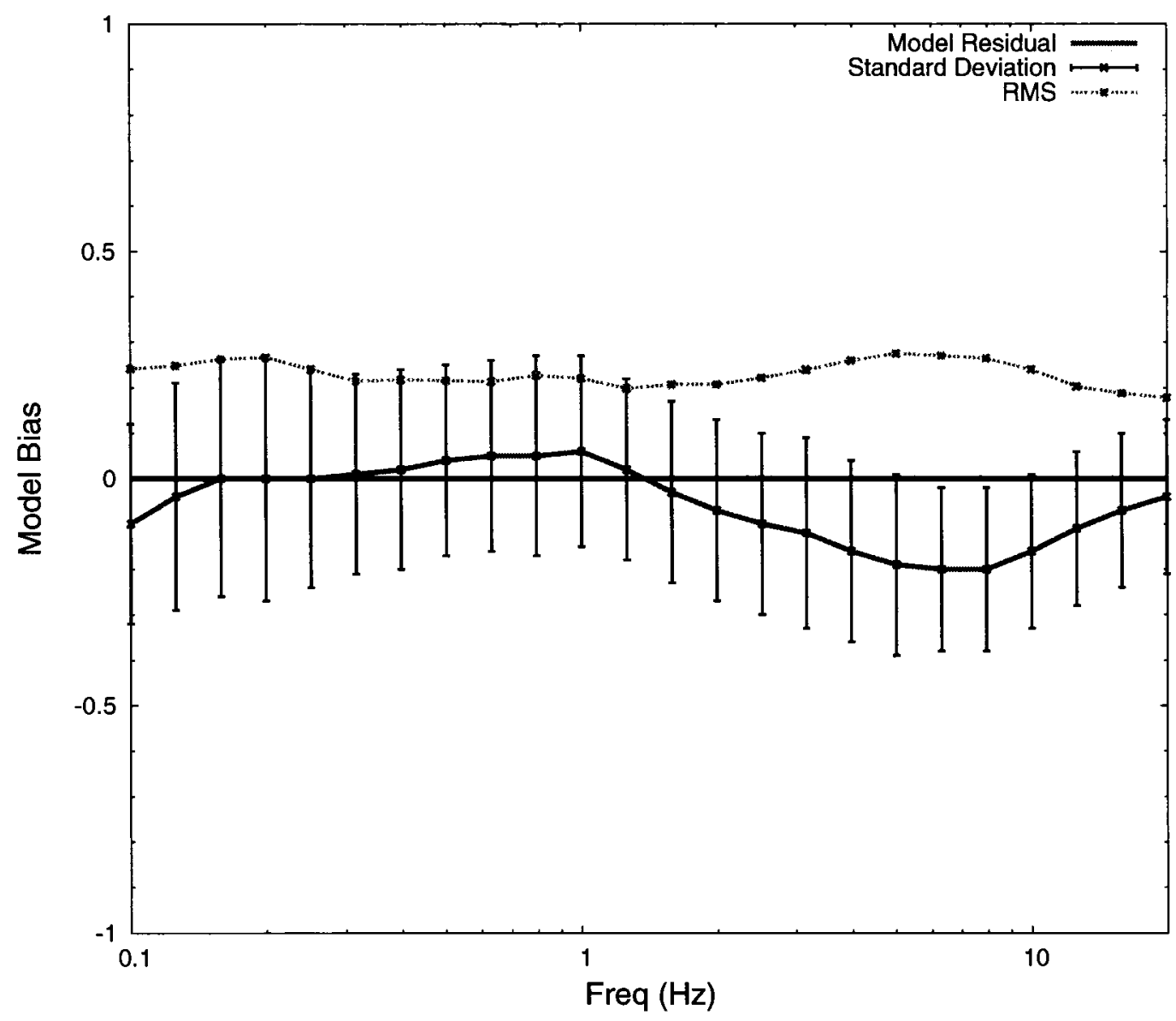

Figure 5.32. Model bias versus frequency for soil stations (NEHRP class D sites) using nonlinear correction by Boore and Atkinson (2005); RMS is the root mean square of the model bias 


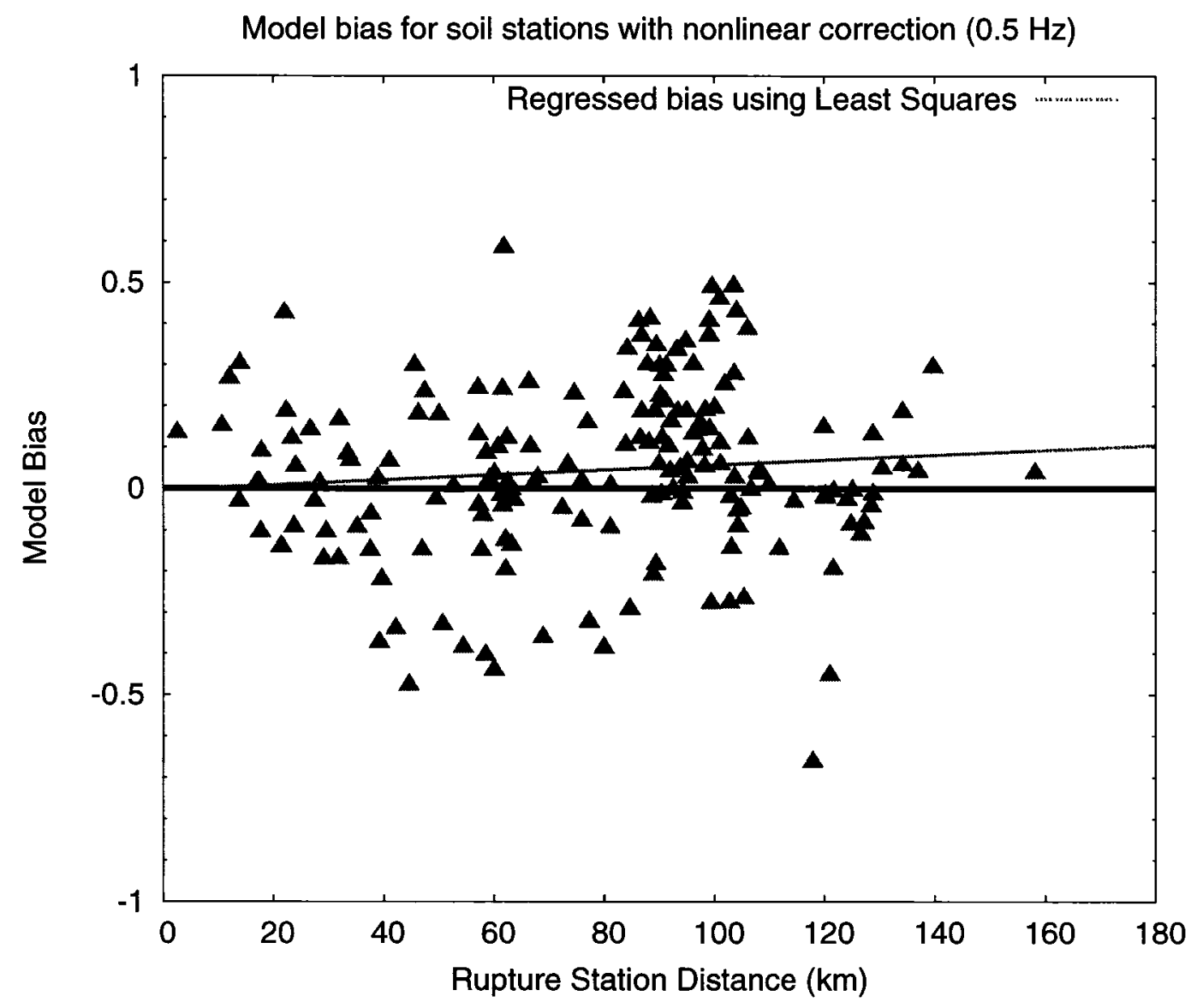

Figure 5.33. Model bias versus distance for soil stations (NEHRP class D sites) after linear and nonlinear correction $(0.5 \mathrm{~Hz})$ using Boore and Atkinson (2005) method 


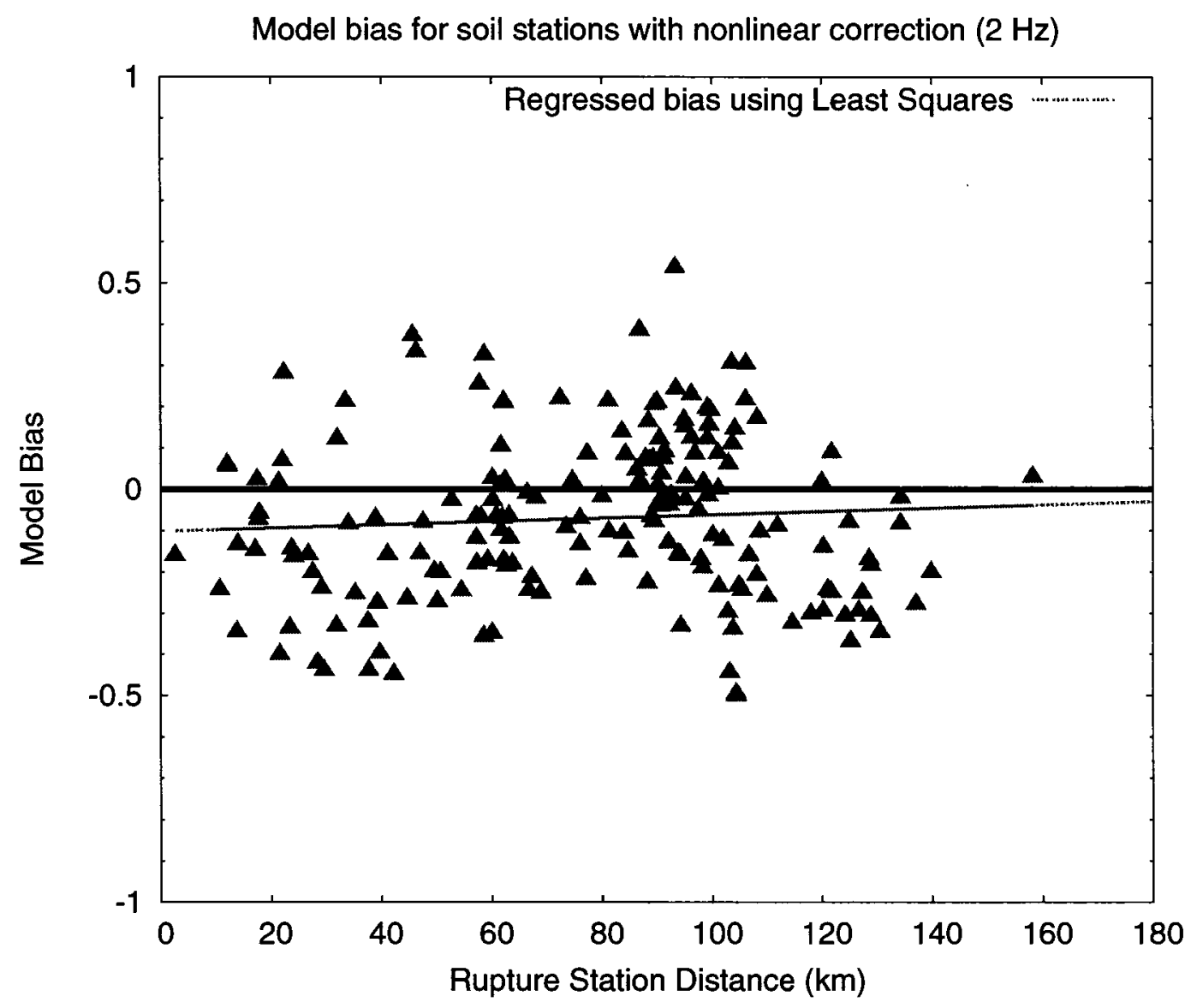

Figure 5.34. Model bias versus distance for soil stations (NEHRP class D sites) after linear and nonlinear correction (2 Hz) using Boore and Atkinson (2005) method 


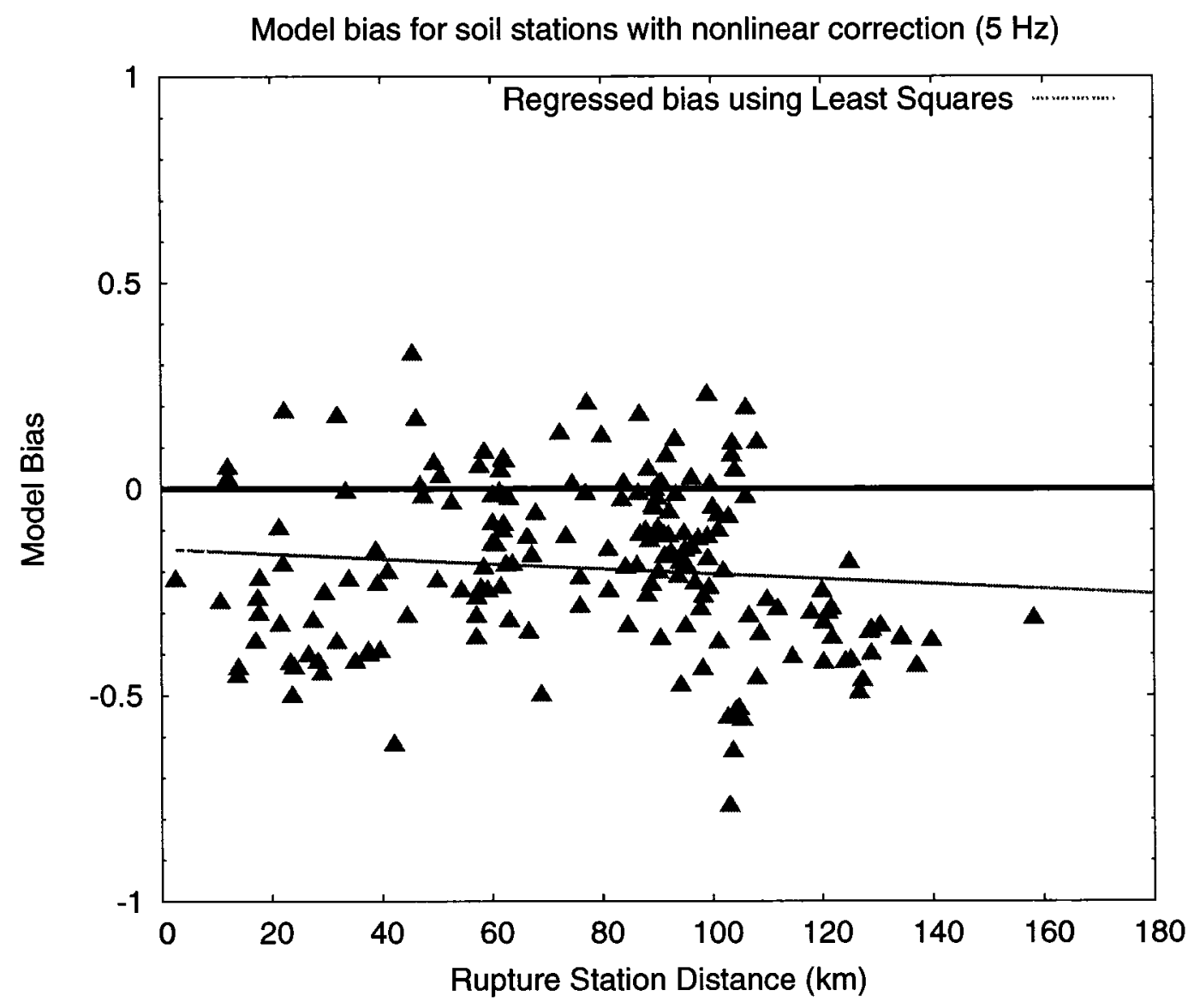

Figure 5.35. Model bias versus distance for soil stations (NEHRP class D sites) after linear and nonlinear correction $(5 \mathrm{~Hz})$ using Boore and Atkinson (2005) method 


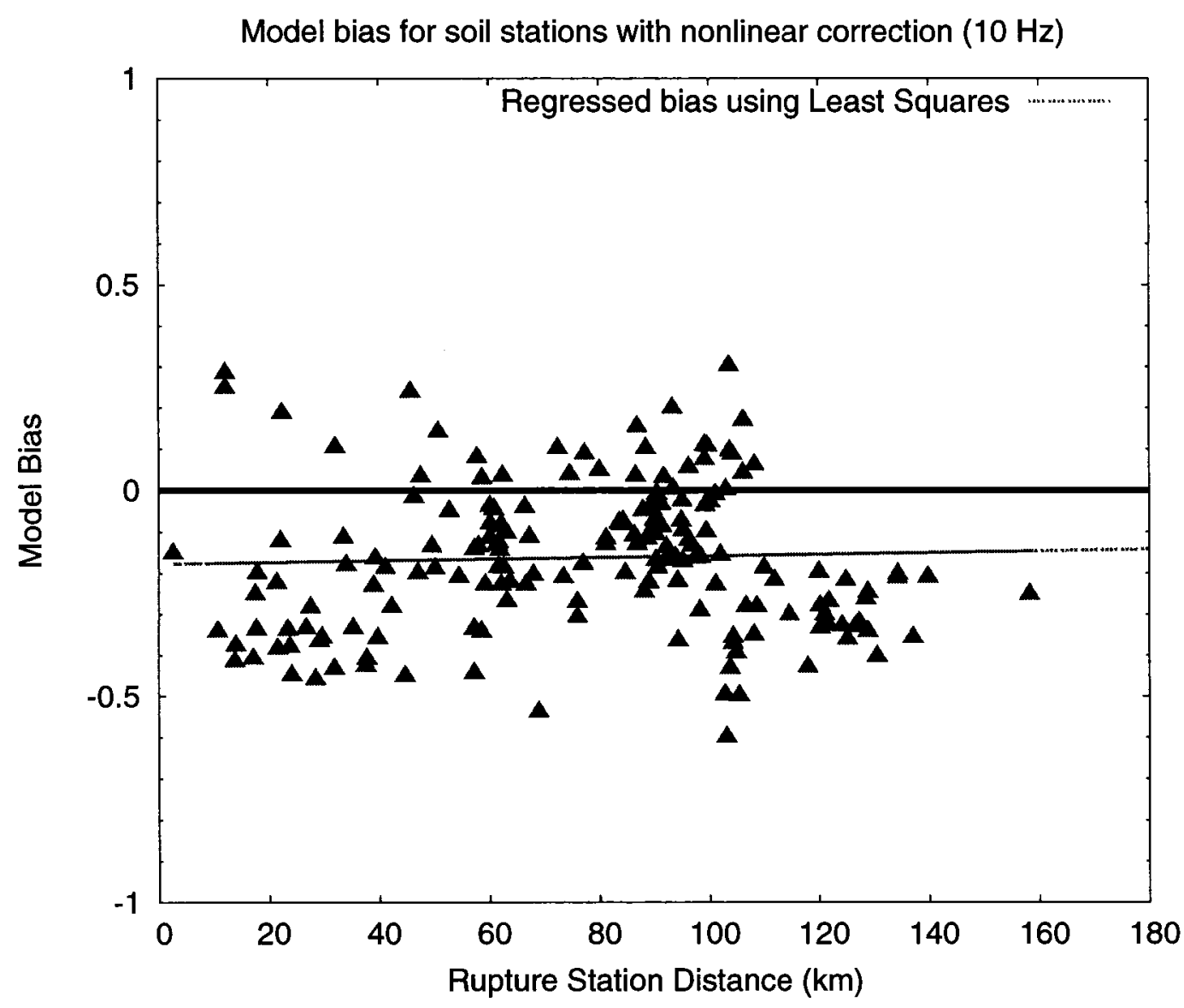

Figure 5.36. Model bias versus distance for soil stations (NEHRP class D sites) after linear and nonlinear correction $(10 \mathrm{~Hz})$ using Boore and Atkinson (2005) method 


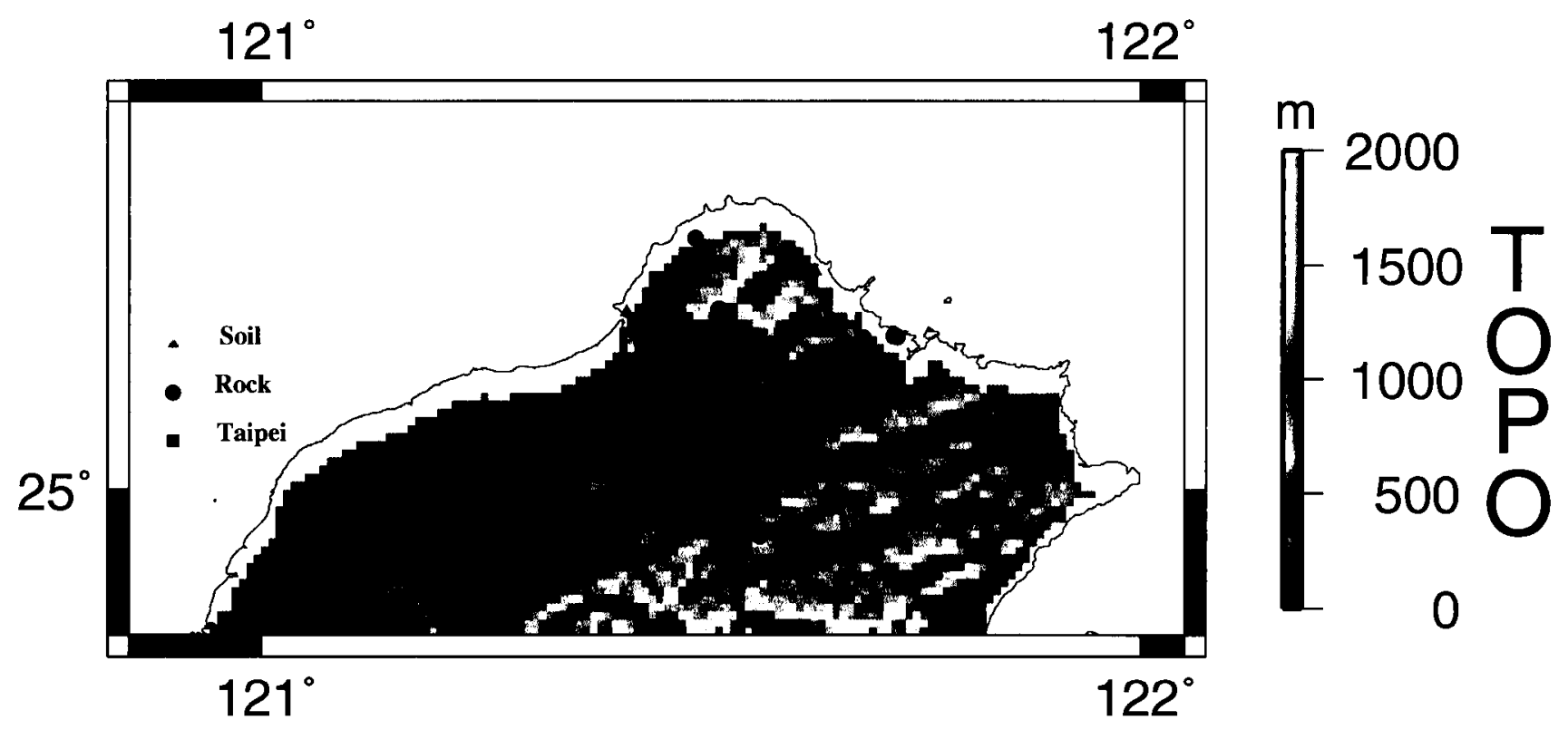

\section{GMT 2005 Dec 12 18:14:12}

Figure 5.37. Topographic map of Taipei region, the up-triangles are NEHRP class D sites while the circles are NEHRP class C sites 


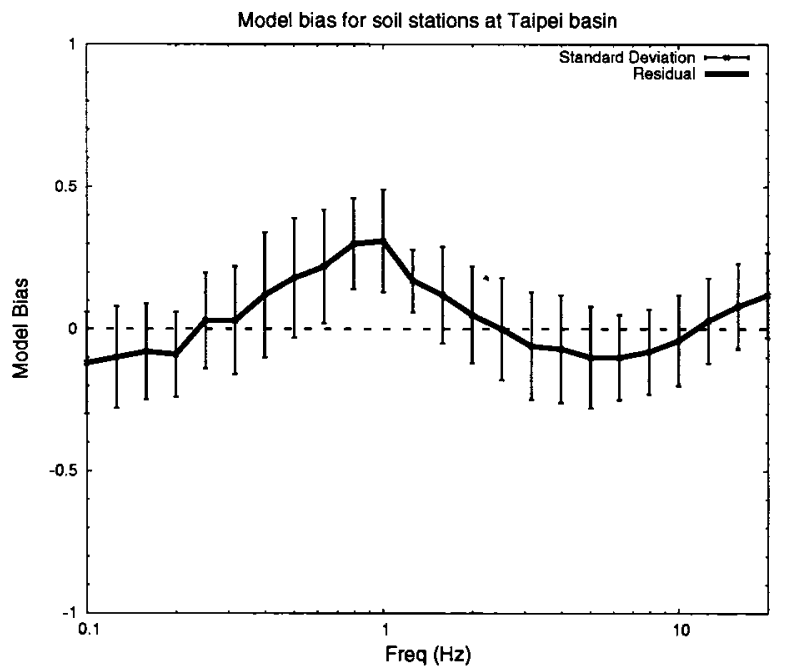

Figure 5.38. Model bias versus frequency for soil stations (NEHRP class D sites) in the Taipei basin using nonlinear correction method by Boore and Atkinson (2005)

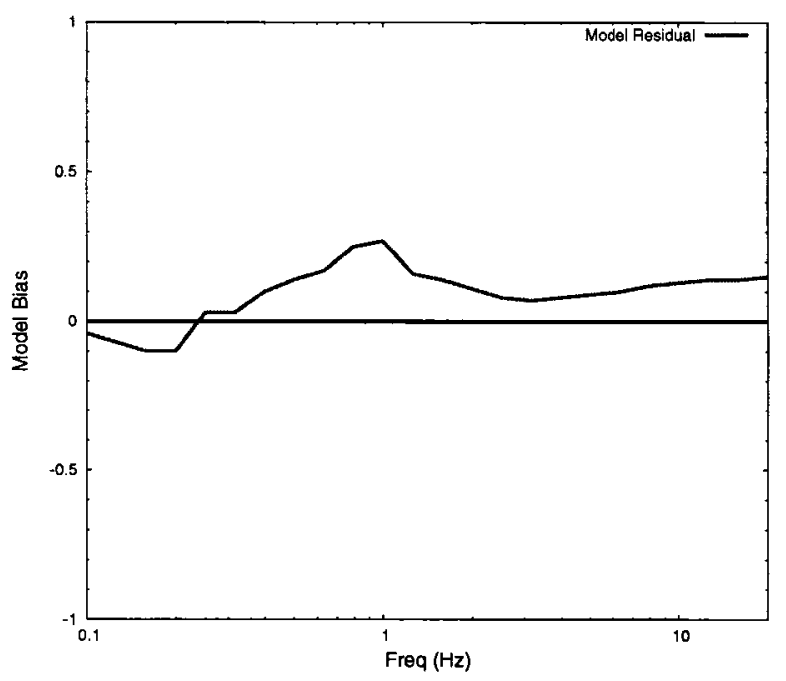

Figure 5.39. Model bias versus frequency for soil stations (NEHRP class D sites) in the Taipei basin using nonlinear correction method by Boore and Atkinson (2005) (This map is the residual in Figure 5.38 minus that of Figure 5.32, demonstrating the net basin effect.) 


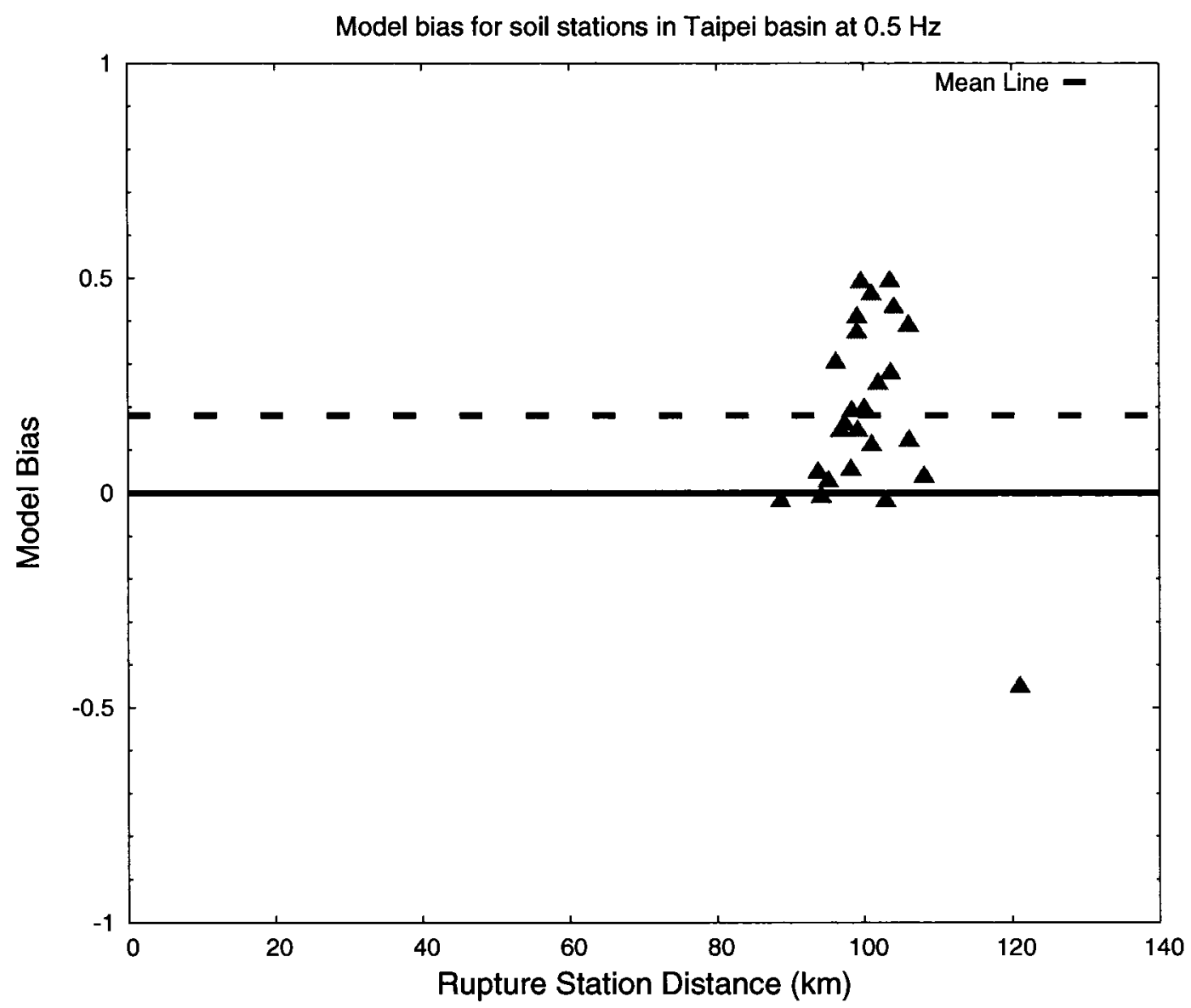

Figure 5.40. Model bias versus distance for soil stations (NEHRP class D sites) in the Taipei basin $(0.5 \mathrm{~Hz})$ using nonlinear correction method by Boore and Atkinson (2005) 


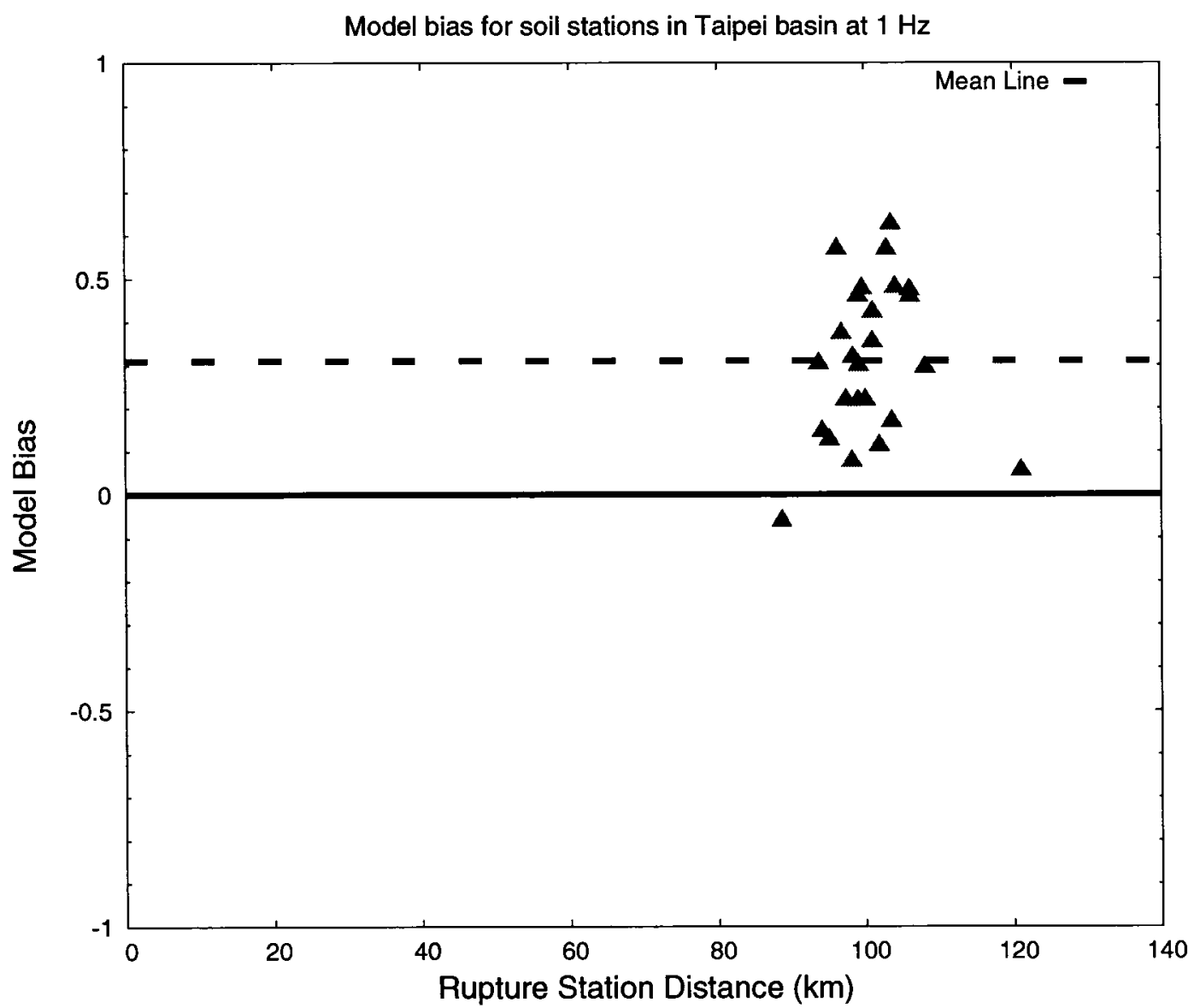

Figure 5.41. Model bias versus distance for soil stations (NEHRP class D sites) in the Taipei basin (1Hz) using nonlinear correction method by Boore and Atkinson (2005) 


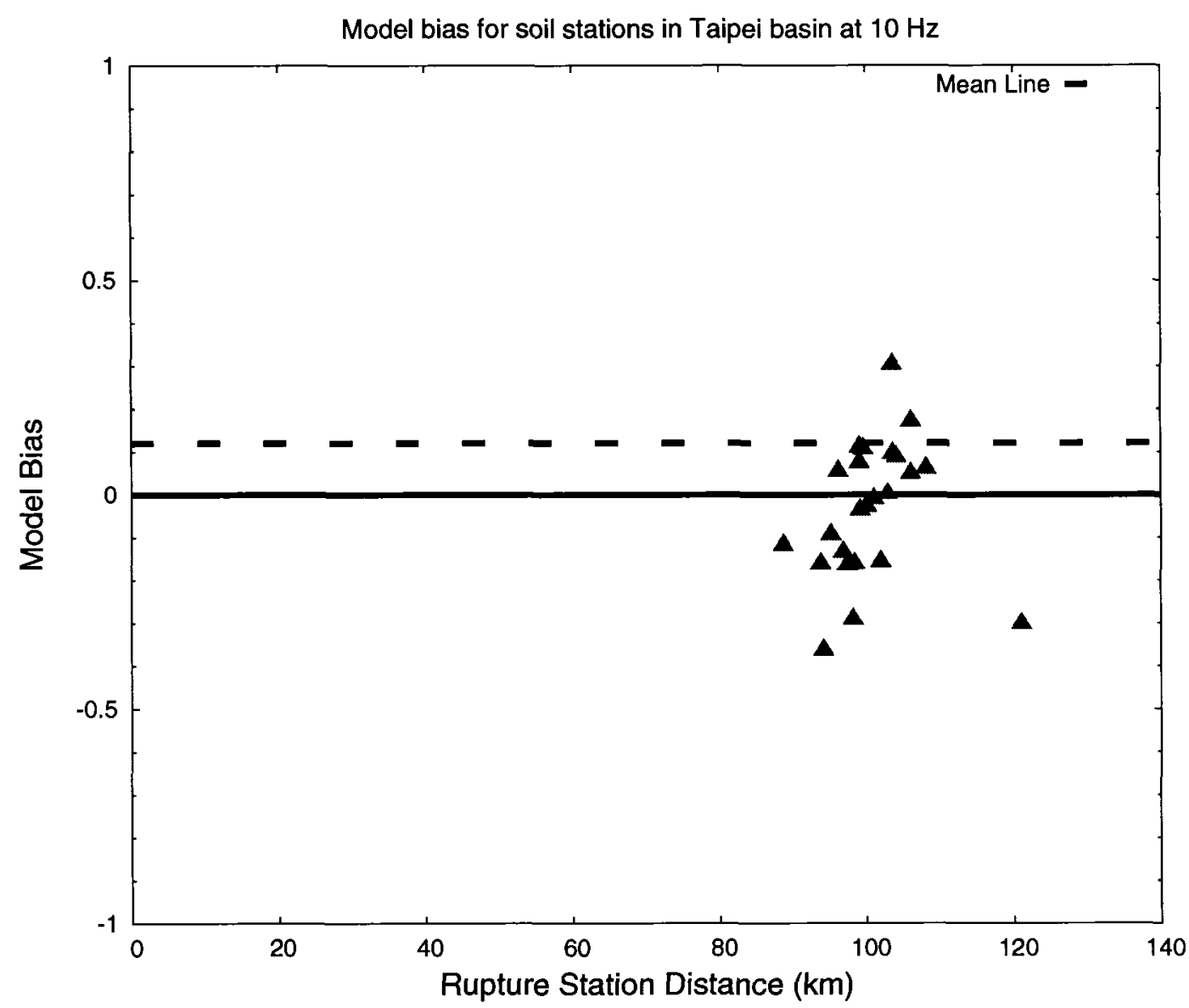

Figure 5.42. Model bias versus distance for soil stations (NEHRP class D sites) in the Taipei basin $(10 \mathrm{~Hz})$ using nonlinear correction method by Boore and Atkinson (2005) 


\section{Chapter 6}

\section{CONCLUSIONS AND FUTURE WORK}

\subsection{Conclusions}

The finite-fault stochastic simulation technique is a robust method which can simulate the dominant characteristics of earthquake ground motions in the frequency and time domains. It has been validated in Puerto Rico (Motazedian, 2002), California (Motazedian and Atkinson, 2005) and then in this study. The seismological model to specify the spectrum of subfaults is the key factor in the stochastic modeling. In this study, most of the model parameters (Seismic wave propagation property, quality factor, high frequency diminution operator, ground motion duration and site amplification function) were first derived empirically using the recorded ground motion data. The model was then applied to the rock stations (NEHRP class C sites) and soil stations (NEHRP class D sites). Comparison of simulated motions to observations leds to the following conclusions:

1. The stress parameter of the 1999 Chi-Chi mainshock, characterizing high-frequency radiation, is about 40 bars. The pulsing percentage of the rupture is above $80 \%$. A stress drop of 40 bars is relatively low compared to typical values of 50 to 70 bars for California earthquakes (eg. (Atkinson and Silva, 1997)).

2. The horizontal $\kappa_{0}$ value is 0.065 for rock and soil stations, which is significantly larger than that for California $\left(\kappa_{0}=0.035\right.$ (Boore and Joyner, 1997)). 
3. The site amplification function based on the quarter wavelength approximation method for NEHRP class C sites of Taiwan is very close to that of California.

4. The $\mathrm{H} / \mathrm{V}$ ratio may also be used as a site amplification function in the simulations. The model bias indicates that the $\mathrm{H} / \mathrm{V}$ ratio approach and the quarter wavelength approximation method are equivalent in terms of overall performance. Thus the $\mathrm{H} / \mathrm{V}$ ratio approach could be an economical alternative to the quarter wavelength approximation method for areas where shear-wave velocity profiles are not available.

5. The slip distribution model has little overall effect on the accuracy of the simulated ground motions but may significantly affect the predicted motions at near-source stations due to the directivity effect.

6. Simulation results suggest significant soil nonlinearity for near-fault sites for the Chi-Chi mainshock for soil stations.

7. Basin effects are observed for soil stations in Taipei basin area, for ground motions of intermediate frequencies (around $1 \mathrm{~Hz}$ ). This could result in significant damage to structures in the Taipei metropolitan region during future great earthquakes and needs to be carefully considered in the building code.

\subsection{Future Work}

The following are possible future research directions for the extended fault simulation technique in Taiwan Island.

- The extended stochastic simulation method can be further validated by the aftershock events of the 1999 Chi-Chi earthquake. By analyzing the aftershock 
records, the attenuation characteristics and quality factor (Q) of Taiwan can be better understood.

- The results of the extended stochastic simulation technique could be improved by including the impulsive behavior of the gourd motion especially for nearsource records, since the impulsive behavior was observed in the 1999 ChiChi earthquake (eg. station TCU052). Methods to include these effects are described by Mavroeidis and Papageorgiou (2003) and incorporated into the EXSIM code of Motazedian and Atkinson (2005).

- The directivity effects of the ground motion could be analyzed by comparison of the durations of simulated seismograms and the recorded ones. 


\section{REFERENCES}

Aki, K. (1966). Generation and propogation of $G$ waves from the Niigata earthquake of June 16, 1964. Part 2. Estimation of earthquake moment, released energy, and stress-strain drop from the $\mathrm{G}$ wave spectrum. Bull. Earthq. Res. Inst. 44, 73-88.

Aki, K. (1967). Scaling law of seismic spectrum. J. Geophys. Res. 72, 1217-1231.

Anderson, J. and Hough, S. (1984). A model for the shape of the Fourier amplitude spectrum of acceleration at high frequency. Bull. Seism. Soc. Am. 74, 1969-1339.

Atkinson, G. (2004). Empirical attenuation of ground-motion spectral amplitudes in southeastern Canada and the northeastern United States. Bull. Seism. Soc. Am. 94, $1079-1095$.

Atkinson, G. and Boore, D. (1995). New ground motion relations for Eastern North America. Bull. Seism. Soc. Am. 85, 17-30.

Atkinson, G. and Boore, D. (1997). Stochastic point-source modeling of ground motions in the Cascadia region. Seism. Res. Lett. 68, 74-85.

Atkinson, G. and Silva, W. (1997). Empirical source spectra for California earthquakes. Bull. Seism. Soc. Am. 87, 97-113.

Atkinson, G. and Silva, W. (2000). Stochastic modeling of California ground motions. Bull. Seism. Soc. Am. 90, 255-274.

Beresnev, I. and Atkinson, G. (1997). Modeling finite fault radiation from the $w^{n}$ spectrum. Bull. Seism. Soc. Am. 87, 67-84.

Beresnev, I. and Atkinson, G. (1998a). FINSIM-a FORTRAN program for simulating stochastic acceleration time histories from finite faults. Seism. Res. Lett. 67, 27-32.

Beresnev, I. and Atkinson, G. (1998b). Stochastic finite-fault modeling of ground motions from the 1994 Northridge, California, earthquake. I. Validation on rock sites. Bull. Seism. Soc. Am. 88, 1392-1401.

Beresnev, I. and Atkinson, G. (1999). Generic finite-fault model for groundmotion prediction in Eastern North America. Bull. Seism. Soc. Am. 89, 608-625. 
Boatwright, J. and Choy, G. (1992). Acceleration source spectra for large earthquakes in northeastern North America. Bull. Seism. Soc. Am. 82, 660-682.

Boore, D. (1983). Stochastic simulation of high-frequency ground motions based on seismological models of the radiated spectra. Bull. Seism. Soc. Am. 73, 1865-1894.

Boore, D. (1996). SMSIM - FORTRAN programs for simulating groundmotions from earthquakes: version 1.0. Open - File Rept. 96-80-A (text) and 96-80-B (diskette) 73P., U.S. Geol. Surv.

Boore, D. and Atkinson, G. (1987). Stochastic prediction of ground motion and spectral response parameters at hard-rock sites in Eastern North America. Bull. Seism. Soc. Am. 77, 440-467.

Boore, D. and Atkinson, G. (2005). Provisional ground-motion prediction equations. Technical report, PEER.

Boore, D. and Joyner, W. (1997). Site amplification for generic rock sites. Bull. Seism. Soc. Am. 87, 327-341.

Boore, D., Joyner, W. and Fumal, T. (1997). Equations for estimating horizontal response spectral and peak acceleration from western North American earthquakes: A summary of recent work. Seism. Res. Lett. 68, 128-153.

Boore, D. M. (2003). Simulation of ground motion using the stochastic method. Pure and Applied Geophysics 160, 635-675.

Borcherdt, R. (1995). Estimates of site-dependent response spectra for design. Earthquake Spectra 10, 617-654.

Bracewell, R. N. (2000). The Fourier transform and its applications. Boston: McGraw Hill.

Brune, J. N. (1970). Tectonic stress and the spectra of seismic shear waves from earthquakes. J. Geophys. Res. 75, 4997-5009.

Castro, R., Rovelli, A., Cocco, M., Di Bona, M. and Pacor, F. (2001). Stochastic simulation of strong-motion records from the 26 September $1997\left(m_{w} 6\right)$, Umbria-Marche (Central Italy) earthquake. Bull. Seism. Soc. Am. 91, 27-39.

CENTRAL GEOLOGICAL SURVEY (MOEA) (2006). General geology and geologic provinces of taiwan. URL: (http://www.moeacgs.gov.tw/english/twgeol/twgeol_generalgeol.jsp). Webpage. 
Central Weather Bureau (Taiwan) (2006). Destruc-

tive earthquakes in the 20th century (1900-1999). URL: (http://scman.cwb.gov.tw/eqv5/damage_list/1900after.htm). Webpage.

Choi, Y. and Stewart, J. (2005). Nonlinear site amplification as function of 30 m shear wave velocity. Earthquake Spectra 21, 1-30.

Fletcher, J. and Wen, K.-L. (2005). Strong ground motion in the Taipei basin from the 1999 Chi-Chi, Taiwan, earthquake. Bull. Seism. Soc. Am. 95, 1428-1446.

Frankel, A., Mueller, C., Barnhard, T., Perkins, D., Leyendecker, E., Dickman, N., S., H. and Hopper, M. (1996). National seismic hazard maps: Documentation. Open-File Rept 69, U.S. Geol. Surv.

Haddon, R. (1996). Earthquake source spectra in Eastern North America. Bull. Seism. Soc. Am. 86, 1300-1313.

Hanks, T. (1982). $f_{\max }$. Bull. Seism. Soc. Am. 72, 1867-1879.

Institute of Earth Sciences, Academia Sinica (Taipei) (2006). Tectonic framework and seismotectonics of taiwan. URL: (http://www.earth.sinica.edu.tw/921/921chichi_tectonics_eng.htm). Webpage.

Irikura, K., Kamae, K. and Dalguer, L. (2000). Source model for simulating ground motion during the 1999 Chi-Chi earthquake. Earthquake Engineering and Engineering Seismology. 2, 1-14.

Kikuchi, M., Yagi, Y. and Yamanaka, Y. (2000). Source process of Chi-Chi, Taiwan earthquake of September 21, 1999 inferred from teleseismic body waves. Bull. Earthq. Res. Inst. Univ. Tokyo 75, 1-13.

Lachet, C. and Bard, P. (1994). Numerical and theoretical invesitigations on the possibilities and limitations of Nakamura's technique. J. Phy. Earth. 42, 377-397.

Lee, C.-T., Cheng, C.-T., Liao, C.-W. and Tsai, Y.-B. (2001a). Site classification of Taiwan free-field strong-motion stations. Bull. Seism. Soc. Am. 91, 1283-1297.

Lee, W., Shin, T., Kuo, K. and Chen, K. (1999). CWB free-field strong-motion data from the 921 Chi-Chi earthquake. Technical report, Seismology Center, Central Weather Bureau, Seismology Center, Central Weather Bureau, Taipei, Taiwan.

Lee, W., Shin, T., Kuo, K., Chen, K. and Fu, C. (2001b). CWB free-field strong-motion data from the 21 September Chi-Chi, Taiwan, earthquake. Bull. Seism. Soc. Am. 2, 134-142. 
Lermo, J. and Chavez-Garcia, F. J. (1994). Are micro-tremors useful in site response evaluation? Bull. Seism. Soc. Am. 84, 1350-1364.

Liu, K.-S. and Tsai, Y.-B. (2005). Attenuation relationship of peak ground acceleration and velocity for crustal earthquakes in Taiwan. Bull. Seism. Soc. Am. 95, 1045-1058.

Mavroeidis, G. P. and Papageorgiou, A. S. (2003). A mathematical representation of near-fault ground motions. Bull. Seism. Soc. Am. 93, 1099-1131.

Motazedian, D. (2002). Development of Earthquake Ground Motion Relations for Puerto Rico. Ph.D. thesis, Carleton University.

Motazedian, D. and Atkinson, G. (2005). Stochastic finite-fault modeling based on a dynamic corner frequency. Bull. Seism. Soc. Am. 95, 995-1010.

Nakamura, Y. (1989). A method for dynamic characteristics estimation of subsurface using micro-tremor on ground surface. $Q R R T R I$. 30, 25-33.

Pacor, F., Cultrera, G., A., M. and Cocco, M. (2005). Finite fault modeling of strong ground motion using a hybrid deterministic-stochastic approach. Bull. Seism. Soc. Am. 95, 225-240.

Papageorgiou, A. and Aki, K. (1983). A specific barrier for the quantitative description of inhomogeneous faulting and the prediction of strong ground motion. II. Application of the model. Bull. Seism. Soc. Am. 73, 953-978.

PEER (2005). Source parameters of 1999 Chi-Chi earthquake. Technical report, PEER.

Phung, V., Atkinson, G. and Lau, D. (2004). Methodology for site classification using strong ground motion data from the 1999 Chi-Chi, Taiwan earthquake. Vancouver, B.C., Canada: 13th World Conference on Earthquake Engineering.

Roumelioti, Z. and Beresnev, I. (2003). Stochastic finite-fault modeling of ground motions from the $1999 \mathrm{Chi}-\mathrm{Chi}$, Taiwan, earthquake: Application to rock and soil sites with implications for nonlinear site response. Bull. Seism. Soc. Am. 93, $1691-1702$.

Roumelioti, Z. and Kiratzi, A. (2002). Stochastic simulation of strong-motion records from the 15 April 1979 (M 7.1) montenegro earthquake. Bull. Seism. Soc. Am. 92, 1095-1101. 
Schneider, J., Silva, W. and Stark, C. (1993). Ground motion model for the 1989 M 6.9 Loma Prieta earthquake including effects of source, path, and site. Earthquake Spectra 9, 251-287.

Shin, T.-C. and Teng, T.-1. (2001). An overview of the 1999 Chi-Chi, Taiwan, earthquake. Bull. Seism. Soc. Am. 91, 895-913.

Sokolov, V. Y., Loh, C.-H. and Wen, K.-L. (2002). Comparison of the Taiwan Chi-Chi earthquake strong-motion data and ground-motion assessment based on spectral model from smaller earthquakes in Taiwan. Bull. Seism. Soc. Am. 92, $1855-1877$.

Wang, W.-H., Chang, S.-H. and Chen, C.-H. (2001). Fault slip inverted from surface displacements during the 1999 Chi-Chi, Taiwan, earthquake. Bull. Seism. Soc. Am. 91, 1167-1181. 


\section{APPENDIX A \\ SAMPLE SIMULATED ACCELEROGRAMS}

The following section demonstrates the comparison of two sample simulated accelerograms and recorded accelerograms (two horizontal components) at station ALS and station HWA056.

- Figure A.1: Station ALS - Near fault station with epicentral distance $38.8 \mathrm{~km}$ and fault-station distance $10.8 \mathrm{~km}$

- Figure A.2: Station HWA056 - Intermediate distance station with epicentral distance $80.3 \mathrm{~km}$ and fault-station distance $41.1 \mathrm{~km}$ : 
Simulated Accelerogram at station ALS (Horizontal)

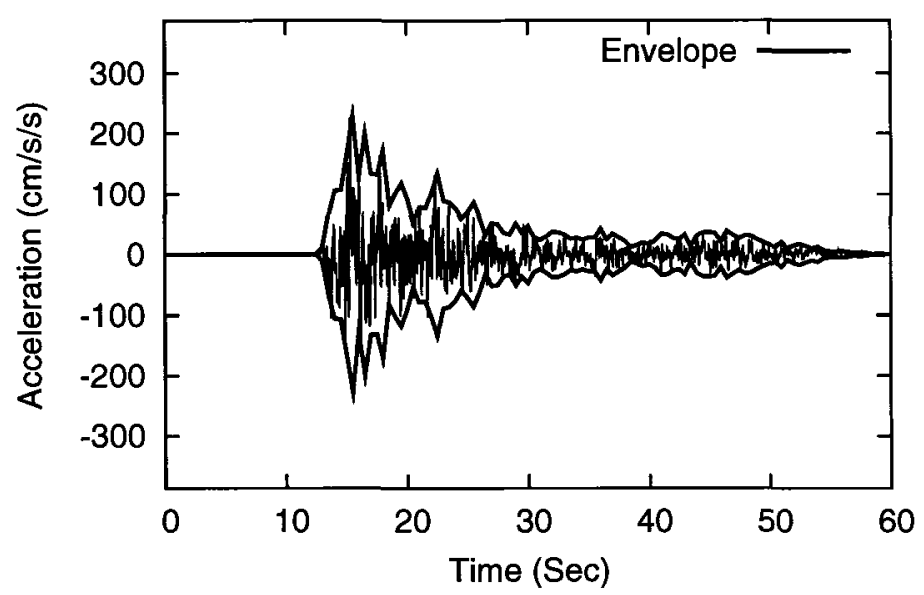

Recorded Accelerogram at station ALS (N-S)

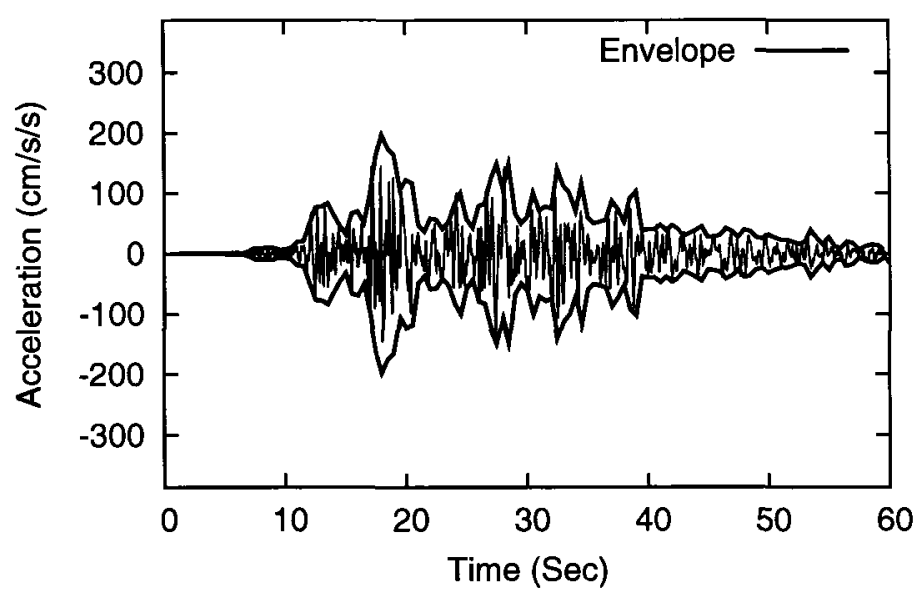

Recorded Accelerogram at station ALS (E-W)

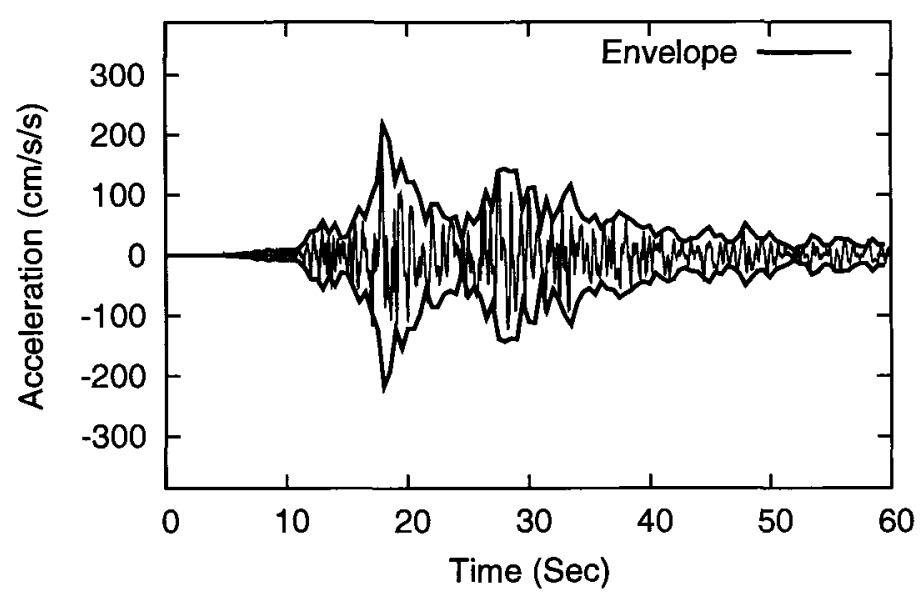

Figure A.1. Comparison of simulated accelerogram and recorded accelerogram at station ALS 


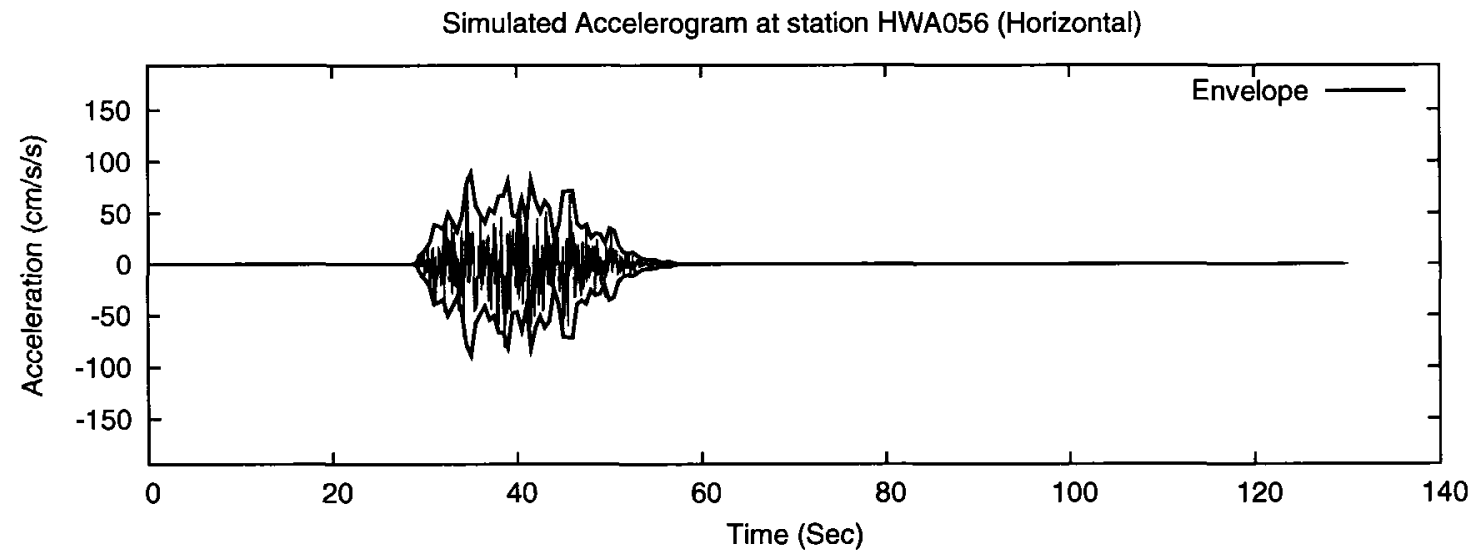

Recorded Accelerogram at station HWA056 (N-S)

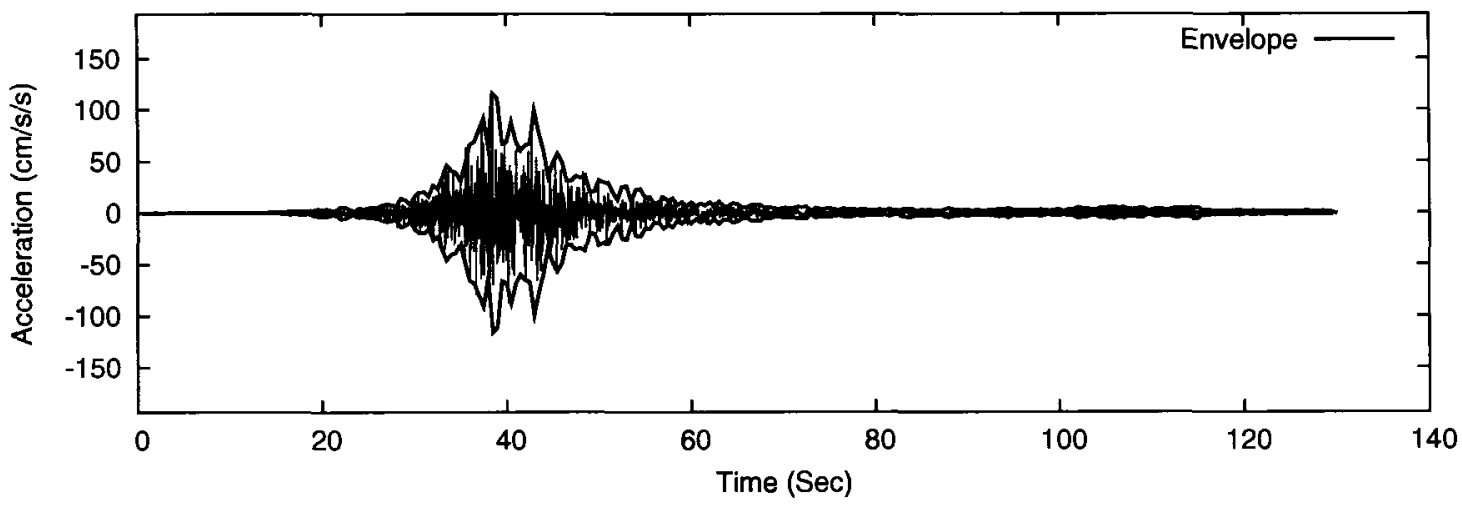

Recorded Accelerogram at station HWA056 (E-W)

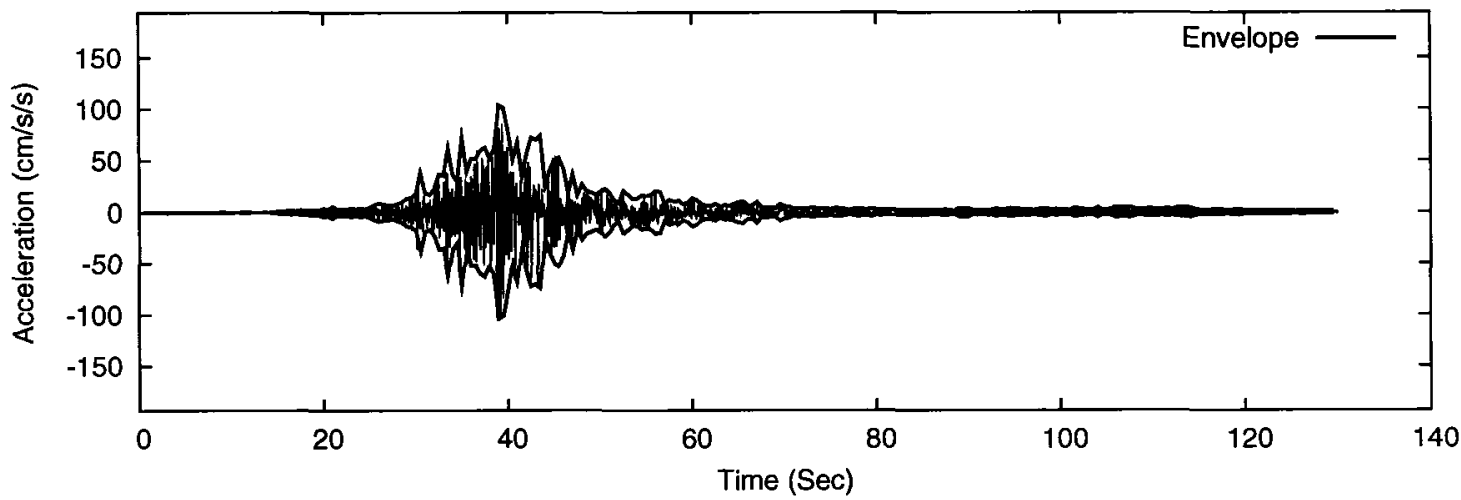

Figure A.2. Comparison of simulated accelerogram and recorded accelerogram at station HWA056 